Georgia State University

ScholarWorks @ Georgia State University

Summer 8-7-2012

\title{
Determining Inclinations of Active Galactic Nuclei via their Narrow-Line Region Kinematics
}

Travis C. Fischer

Georgia State University

Follow this and additional works at: https://scholarworks.gsu.edu/phy_astr_diss

\section{Recommended Citation}

Fischer, Travis C., "Determining Inclinations of Active Galactic Nuclei via their Narrow-Line Region Kinematics." Dissertation, Georgia State University, 2012.

doi: https://doi.org/10.57709/3097087

This Dissertation is brought to you for free and open access by the Department of Physics and Astronomy at ScholarWorks @ Georgia State University. It has been accepted for inclusion in Physics and Astronomy Dissertations by an authorized administrator of ScholarWorks @ Georgia State University. For more information, please contact scholarworks@gsu.edu. 


\title{
DETERMINING INCLINATIONS OF ACTIVE GALACTIC NUCLEI VIA THEIR NARROW-LINE REGION KINEMATICS
}

\author{
by \\ TRAVIS CODY FISCHER \\ Under the Direction of D. Michael Crenshaw
}

\begin{abstract}
Active Galactic Nuclei (AGN) are axisymmetric systems to first order; their observed properties are likely strong functions of inclination with respect to our line of sight. However, except for a few special cases, the specific inclinations of individual AGN are unknown. We have developed a promising technique for determining the inclinations of nearby AGN by mapping the kinematics of their narrow-line regions (NLRs), which are easily resolved with Hubble Space Telescope (HST) [O III] imaging and long-slit spectra from the Space Telescope Imaging Spectrograph (STIS). Our studies indicate that NLR kinematics dominated by radial outflow can be fit with simple biconical outflow models that can be used to determine the inclination of the bicone axis, and hence the obscuring torus, with respect to our line of sight. We present NLR analysis of 52 Seyfert galaxies and resultant inclinations from models of 17 individual AGN with clear signatures of biconical outflow. From these AGN, we can for the first time assess the effect of inclination on other observable properties in radio-quiet AGN, including the discovery of a distinct correlation between AGN inclination and X-ray column density.
\end{abstract}

INDEX WORDS: $\quad$ AGN, Seyfert galaxies, NLR, Outflows, Kinematics, Bicones, Unified Model 
DETERMINING INCLINATIONS OF ACTIVE GALACTIC NUCLEI VIA THEIR NARROW-LINE REGION KINEMATICS

by

TRAVIS CODY FISCHER

A Dissertation Submitted in Partial Fulfillment of Requirements for the Degree of

Doctor of Philosophy

in the College of Arts and Sciences

Georgia State University 
Copyright by

Travis C. Fischer

2012 
DETERMINING INCLINATIONS OF ACTIVE GALACTIC NUCLEI VIA THEIR NARROW-LINE REGION KINEMATICS

by

\section{TRAVIS CODY FISCHER}

Committee Chair:
Dommittee:
H. Richard Miller
Misty C. Bentz
Russel J. White
Paul J. Wiita
Henrique R. Schmitt
Mukesh Dhamala

Electronic Version Approved:

Office of Graduate Studies

College of Arts and Sciences

Georgia State University

August 2012 


\section{DEDICATION}

I would like to dedicate this work to my unyieldingly proud parents and my unyieldingly loving fiancee. 


\section{ACKNOWLEDGMENTS}

I would like to thank the faculty and staff of the Georgia State University Astronomy Program for allowing me to attend this university and providing aid in my young academic career. The histograms in this work were made quickly with the help of Jen Winters. Thanks to Dr. Russel White for insightful Friday conversations. I wish to thank my constant collaborators, Drs. Steve Kraemer and Henrique Schmitt, for all of their direction and assistance throughout this work. Most especially, I would like to thank my advisor, Dr. Mike Crenshaw. He is the man and I am forever grateful for his guidance and counsel. 


\section{TABLE OF CONTENTS}

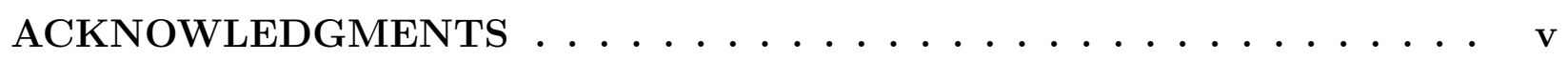

LIST OF TABLES ...................... . .

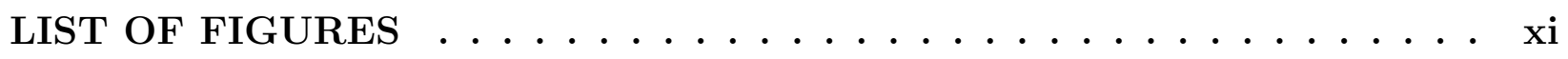

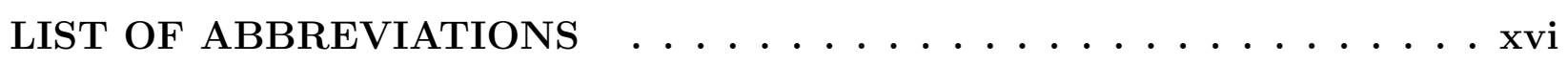

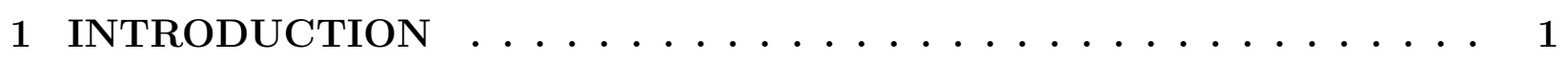

1.1 Seyfert Galaxy Components . . . . . . . . . . . . . . . . 2

1.2 The Unified Model . . . . . . . . . . . . . . . . . . 3

1.3 The Narrow-Line Region . . . . . . . . . . . . . . . . . . . . . 4

1.4 Outflows in the NLR ...................... 5

1.5 Motivation for the Current Study . . . . . . . . . . . . . . . 7

2 OBSERVATIONS AND ANALYSIS ................ 8

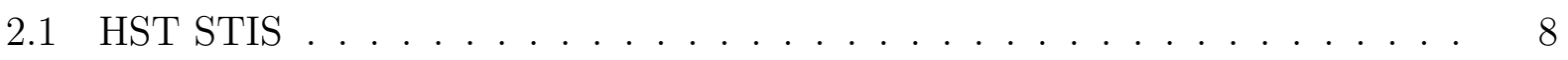

2.2 Line Analysis . . . . . . . . . . . . . . . . . . . . . 9

2.3 Calculating Velocities . . . . . . . . . . . . . . . . . 10

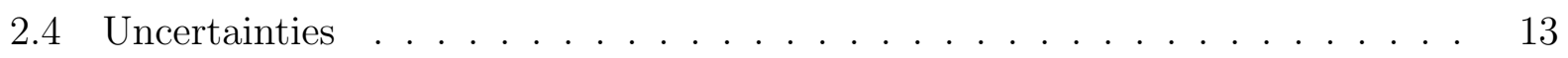

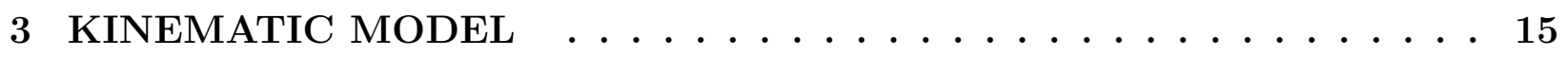

3.1 General Assumptions . . . . . . . . . . . . . . . . . . 15

3.2 Model Parameters . . . . . . . . . . . . . . . . . . 16

3.3 Creating the Model . . . . . . . . . . . . . . . . . 17

3.4 Fitting the Model to Data . . . . . . . . . . . . . . . 18

3.5 Incorporating the Host Disk . . . . . . . . . . . . . . . . . . . 20 


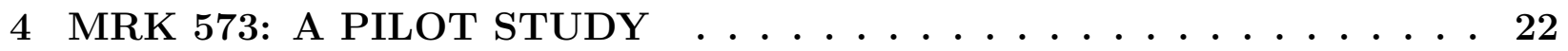

4.1 Mrk 573: Observations . . . . . . . . . . . . . . . . . . 23

4.2 Mrk 573: Results . . . . . . . . . . . . . . . . . . . . 24

4.3 Mrk 573: Models . . . . . . . . . . . . . . . . . . 27

4.4 Mrk 573: Discussion . . . . . . . . . . . . . . . . . 31

4.5 Mrk 573: Conclusions . . . . . . . . . . . . . . . . . . . . . 34

5 MRK 78: AN APPLICATION OF OUTFLOW MODELING $\ldots \ldots . .36$

5.1 Mrk 78: Observations . . . . . . . . . . . . . . . . . . . . . . 37

5.2 Mrk 78: Results . . . . . . . . . . . . . . . . . . . 40

$5.3 \quad$ Mrk 78: Models . . . . . . . . . . . . . . . . . . . . . 43

5.4 Mrk 78: Discussion . . . . . . . . . . . . . . . . . 45

$5.5 \quad$ Mrk 78: Conclusions . . . . . . . . . . . . . . . . . . . . . 49

6 EXPANDING THE SEYFERT AGN SAMPLE $\ldots \ldots \ldots \ldots$

6.1 Observations. . . . . . . . . . . . . . . . . . . 50

6.1 .1 MAST Archive Spectra . . . . . . . . . . . . . . . 50

6.1 .2 Proprietary Spectra . . . . . . . . . . . . . . . . . . . . 50

$6.1 .3 \quad$ Slitless Spectra _. . . . . . . . . . . . . . . . . 52

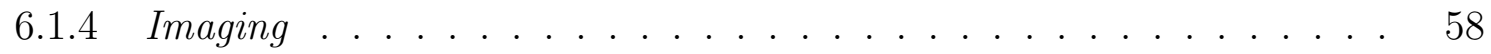

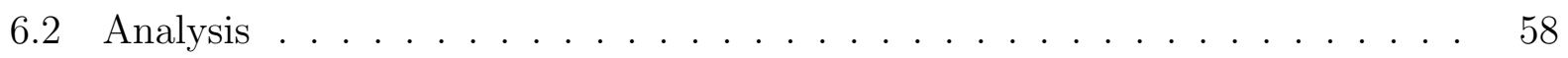

6.3 Targets With Clear Outflow Signatures . . . . . . . . . . . . . . 62

6.3 .1 Circinus ............................. 62

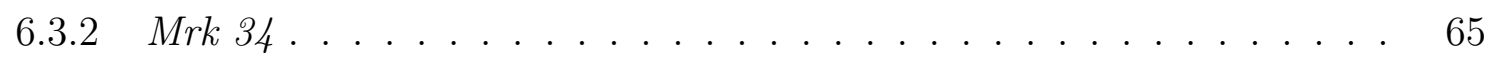

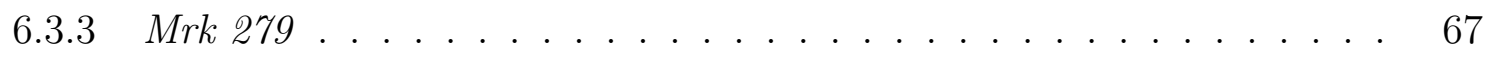

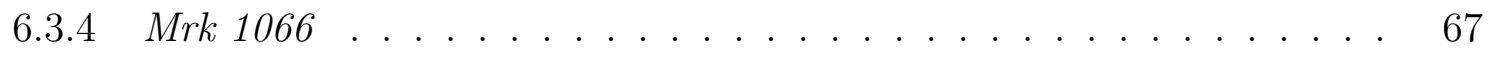

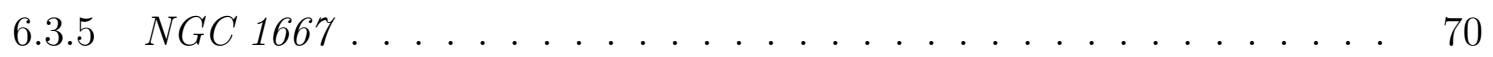

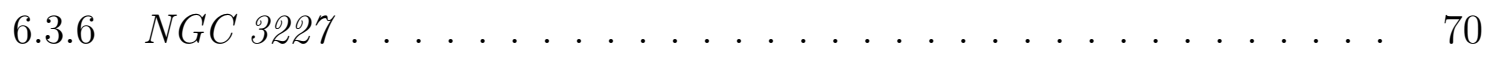

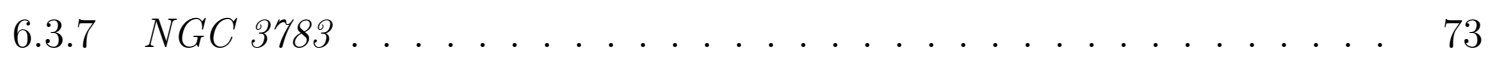

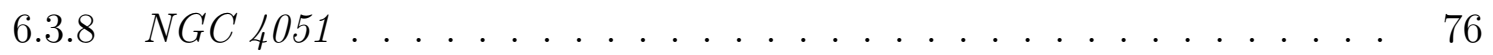

$6.3 .9 \quad N G C 4507 \ldots \ldots \ldots \ldots \ldots \ldots$

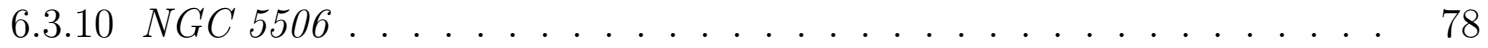




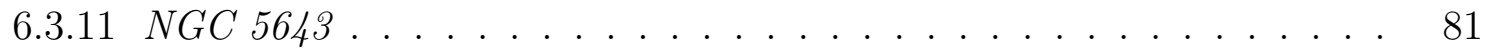

$6.3 .12 N G C 7674 \ldots \ldots . \ldots \ldots$

6.4 Distinct Unmodeled Targets . . . . . . . . . . . . . . . . . . . . . . . 83

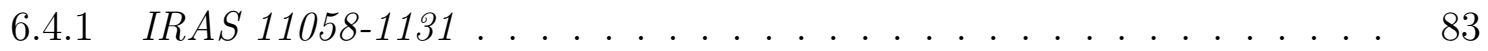

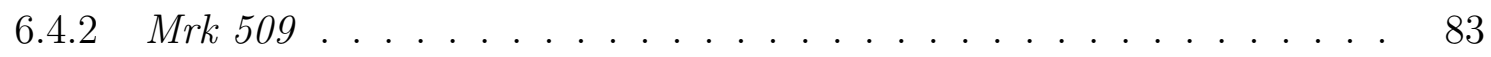

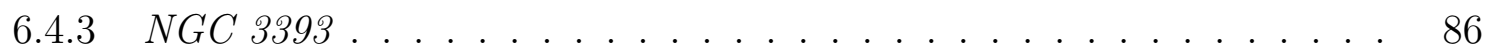

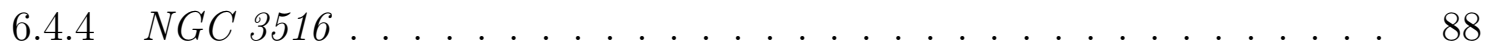

7 EXPANDED SAMPLE RESULTS . . . . . . . . . . . . . 91

7.1 Column Density . . . . . . . . . . . . . . . . . . 92

7.2 Mid-IR Flux . . . . . . . . . . . . . . . . . . . . . . . . . 96

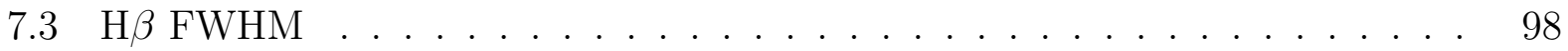

7.4 Possible Correlations . . . . . . . . . . . . . . . . . . . . 102

7.5 Non-Correlations . . . . . . . . . . . . . . . . . 103

7.6 Discussion . . . . . . . . . . . . . . . . . . . . 104

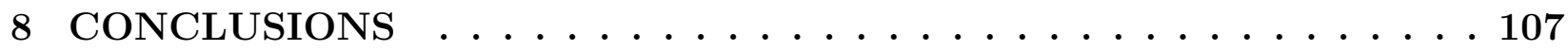

8.1 Lessons Learned . . . . . . . . . . . . . . . . . . . . . . . . 107

8.1.1 In Situ Acceleration in NLRs . . . . . . . . . . . . . . . . . . . . 107

8.1.2 Double Peaked Emission Lines Do Not Necessarily Equal Double AGN 107

8.1.3 Inclination Dependent AGN Properties . . . . . . . . . . . . . . . . . 107

8.2 Future Work . . . . . . . . . . . . . . . . . . . . . . 109

8.2.1 Increase the Sample of AGN Inclinations . . . . . . . . . . . . . . . . 109

8.2.2 Determine the Multiwavelength Properties of the AGN Sample . . . . 111

REFERENCES ........................ 112

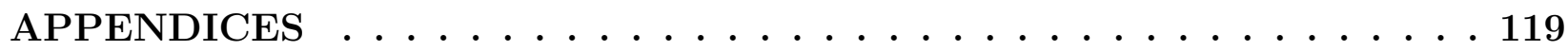

A Extended Sample: HST STIS Slit Positions . . . . . . . . . 119

B Extended Sample: Spectral Images . . . . . . . . . . . 131 
C Extended Sample: AGN Kinematics Data . . . . . . . . . . . 148

D Extended Sample: AGN Kinematic Models . . . . . . . . . . . 183 


\section{LIST OF TABLES}

Table $2.1 \quad H S T /$ STIS Grating Specifications . . . . . . . . . . . . . . 8

Table 2.2 Maximum Errors In Velocity Measurements . . . . . . . . . . . . . . . 14

Table 3.1 Parameter Abbreviations . . . . . . . . . . . . . . 16

Table $4.1 \quad H S T /$ STIS Observations of Mrk $573 \ldots \ldots$. . . . . . . . . 25

Table 4.2 Final Parameters for Mrk $573 \ldots \ldots$. . . . . . . . . . . . 31

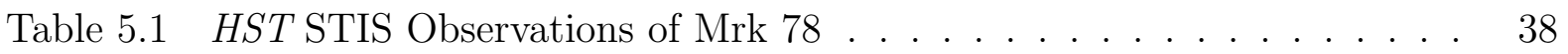

Table $5.2 \quad H S T$ Imaging Observations of Mrk 78 . . . . . . . . . . . . . . . 39

Table 5.3 Final Parameters for Mrk 78 . . . . . . . . . . . . . . . . . . . . 44

Table 6.1 Expanded Sample: HST/STIS Observations . . . . . . . . . . . 53

Table 6.2 Expanded Sample: HST Imaging Observations and Redshifts . . . . . 59

Table 6.3 Total Sample AGN Classifications . . . . . . . . . . . . . . . 62

Table 6.4 Total Sample Modeled AGN Parameters . . . . . . . . . . . . . . . 64

Table 7.1 Physical Properties Of Modeled AGN Sample . . . . . . . . . . . . 92

Table 7.2 Mid-IR Fluxes Of Modeled AGN Sample . . . . . . . . . . . . . . . 99 


\section{LIST OF FIGURES}

Figure 1.1 Picture of an AGN showing the major components . . . . . . . . 3

Figure 2.1 Example Spectral Image of Mrk $34 \ldots \ldots$. . . . . . . . . . 9

Figure 2.2 Example Gaussian Fits to Spectra . . . . . . . . . . . . . . . . . . 11

Figure 2.3 Example Radial Velocites, FWHMs, Fluxes . . . . . . . . . . . . . . 12

Figure 3.1 Toy Kinematic Model . . . . . . . . . . . . . . . . . . . . . 16

Figure 3.2 Example Kinematic Model . . . . . . . . . . . . . . . . . . . . . 19

Figure 4.1 Mrk 573 Slit Position . . . . . . . . . . . . . . . . . . 24

Figure $4.2 \quad H S T /$ STIS long-slit spectral images of Mrk 573 . . . . . . . . . . . . 25

Figure 4.3 Mrk 573 Kinematics . . . . . . . . . . . . . . . . . . . . 26

Figure 4.4 Mrk 573 Kinematic Model . . . . . . . . . . . . . . . . . . . . 28

Figure 4.5 Mrk 573 Geometric Model . . . . . . . . . . . . . . . . . . 30

Figure 4.6 Mrk 573 Geometric Model + Imaging . . . . . . . . . . . . . . . 30

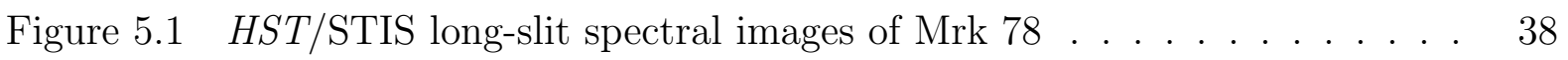

Figure 5.2 Mrk 78 Slit Positions . . . . . . . . . . . . . . . . . . . . . . . 39

Figure 5.3 Mrk 78 Pseudo Ground-Based Obseration . . . . . . . . . . . . . . 41

Figure 5.4 Mrk 78 Kinematics . . . . . . . . . . . . . . . . . . . . 42

Figure 5.5 Mrk 78 Kinematic Model . . . . . . . . . . . . . . . . . . . . 45

Figure 5.6 Mrk 78 Geometric Models . . . . . . . . . . . . . . . . . . . 47

Figure 6.1 Seyfert Type Modeling Bias Histogram ～. . . . . . . . . . . . . 60

Figure 6.2 Redshift Modeling Bias Histogram ． . . . . . . . . . . . . . . 61

Figure 6.3 Circinus Kinematic and Geometric Models . . . . . . . . . . . 63

Figure 6.4 Mrk 34 Kinematic and Geometric Models . . . . . . . . . . . . . . 66

Figure 6.5 Mrk 279 Kinematic and Geometric Models . . . . . . . . . . . . 68

Figure 6.6 Mrk 1066 Kinematic and Geometric Models . . . . . . . . . . . . . . 69 
Figure 6.7 NGC 1667 Kinematic and Geometric Models . . . . . . . . . . . . 71

Figure 6.8 NGC 3227 Kinematic and Geometric Models . . . . . . . . . . . . . . 72

Figure 6.9 NGC 3783 Kinematic and Geometric Models . . . . . . . . . . . . . . 74

Figure 6.10 NGC 3783: Müller-Sánchez et al. (2011) Model . . . . . . . . . . . . 75

Figure 6.11 NGC 4051 Kinematic and Geometric Models . . . . . . . . . . . . . 77

Figure 6.12 NGC 4507 Kinematic and Geometric Models . . . . . . . . . . . . . 79

Figure 6.13 NGC 5506 Kinematic and Geometric Models . . . . . . . . . . . . . 80

Figure 6.14 NGC 5643 Kinematic and Geometric Models . . . . . . . . . . . . . . 82

Figure 6.15 NGC 7674 Kinematic and Geometric Models . . . . . . . . . . . . . . 84

Figure 6.16 Mrk 509 Kinematics . . . . . . . . . . . . . . . . . . 85

Figure 6.17 NGC 3393 Kinematics . . . . . . . . . . . . . . . . . 86

Figure 6.18 NGC 3393 Kinematic Model . . . . . . . . . . . . . . . . . 88

Figure 6.19 NGC 3516 Kinematics . . . . . . . . . . . . . . . . . . . . . . 89

Figure 7.1 Inclination Histogram . . . . . . . . . . . . . . . . . . . 93

Figure 7.2 Outer Opening Angle Histogram . . . . . . . . . . . . . . . . . . . . 94

Figure 7.3 Inclination vs. Column Density . . . . . . . . . . . . . . 96

Figure 7.4 Mid-IR Flux Measurement . . . . . . . . . . . . . . . . . . . 98

Figure 7.5 Inclination vs. Mid-IR Color . . . . . . . . . . . . . . . . . . 100

Figure 7.6 Inclination vs. $\mathrm{H} \beta$ FWHM . . . . . . . . . . . . . . . . . . . 101

Figure 7.7 Bolometric Luminosity vs Maximum Velocity . . . . . . . . . . . . . 102

Figure 7.8 Bolometric Luminosity vs. Bicone Height . . . . . . . . . . . . . . . 103

Figure A.1 HST STIS Slit Positions . . . . . . . . . . . . . . . . . . 119

Figure A.2 HST STIS Slit Positions . . . . . . . . . . . . . . . . . . . 120

Figure A.3 HST STIS Slit Positions . . . . . . . . . . . . . . . . . . . . . 121

Figure A.4 HST STIS Slit Positions . . . . . . . . . . . . . . . . . . . . . 122

Figure A.5 HST STIS Slit Positions . . . . . . . . . . . . . . . . . . . 123

Figure A.6 HST STIS Slit Positions . . . . . . . . . . . . . . . . . . . . . . 124

Figure A.7 HST STIS Slit Positions _ . . . . . . . . . . . . . . . 125 
Figure A.8 HST STIS Slit Positions _ . . . . . . . . . . . . . . . . . . 126

Figure A.9 HST STIS Slit Positions . . . . . . . . . . . . . . . . . . . . . . . . . 127

Figure A.10 HST STIS Slit Positions . . . . . . . . . . . . . . . . . . . . . . 128

Figure A.11 HST STIS Slit Positions . . . . . . . . . . . . . . . . . . . . . . . . . 129

Figure A.12 HST STIS Slit Positions . . . . . . . . . . . . . . . . . . . . . 130

Figure B.1 HST STIS Spectral Images . . . . . . . . . . . . . . . . . . . . . . . . 132

Figure B.2 HST STIS Spectral Images . . . . . . . . . . . . . . . . . . . 133

Figure B.3 HST STIS Spectral Images . . . . . . . . . . . . . . . . . . . . . . . . 134

Figure B.4 HST STIS Spectral Images . . . . . . . . . . . . . . . . . . . . 135

Figure B.5 HST STIS Spectral Images . . . . . . . . . . . . . . . . . . . 136

Figure B.6 HST STIS Spectral Images . . . . . . . . . . . . . . . . . . 137

Figure B.7 HST STIS Spectral Images . . . . . . . . . . . . . . . . . . . . . . 138

Figure B.8 HST STIS Spectral Images . . . . . . . . . . . . . . . . . . . . 139

Figure B.9 HST STIS Spectral Images . . . . . . . . . . . . . . . . . . . . . 140

Figure B.10 HST STIS Spectral Images . . . . . . . . . . . . . . . . . . . . . 141

Figure B.11 HST STIS Spectral Images . . . . . . . . . . . . . . . . . . . . . . . . 142

Figure B.12 HST STIS Spectral Images . . . . . . . . . . . . . . . . . . . . . . 143

Figure B.13 HST STIS Spectral Images . . . . . . . . . . . . . . . . . . . . . 144

Figure B.14 HST STIS Spectral Images . . . . . . . . . . . . . . . . . . . . . . 145

Figure B.15 HST STIS Spectral Images . . . . . . . . . . . . . . . . . . 146

Figure B.16 HST STIS Spectral Images . . . . . . . . . . . . . . . . . . . . 147

Figure C.1 Expanded Sample Kinematics . . . . . . . . . . . . . . . . . . . . 149

Figure C.2 Expanded Sample Kinematics . . . . . . . . . . . . . . . . . . . . . 150

Figure C.3 Expanded Sample Kinematics . . . . . . . . . . . . . . . . . 151

Figure C.4 Expanded Sample Kinematics . . . . . . . . . . . . . . . . . . . 152

Figure C.5 Expanded Sample Kinematics . . . . . . . . . . . . . . . . . . 153

Figure C.6 Expanded Sample Kinematics . . . . . . . . . . . . . . . . . . . . . 154

Figure C.7 Expanded Sample Kinematics . . . . . . . . . . . . . . . . 155 
Figure C.8 Expanded Sample Kinematics . . . . . . . . . . . . . . . . 156

Figure C.9 Expanded Sample Kinematics . . . . . . . . . . . . . . . . . 157

Figure C.10 Expanded Sample Kinematics . . . . . . . . . . . . . . . . . . . . 158

Figure C.11 Expanded Sample Kinematics . . . . . . . . . . . . . . . . . . . 159

Figure C.12 Expanded Sample Kinematics . . . . . . . . . . . . . . . . . . . 160

Figure C.13 Expanded Sample Kinematics . . . . . . . . . . . . . . . . . 161

Figure C.14 Expanded Sample Kinematics . . . . . . . . . . . . . . . . . . . . . 162

Figure C.15 Expanded Sample Kinematics . . . . . . . . . . . . . . . . . 163

Figure C.16 Expanded Sample Kinematics . . . . . . . . . . . . . . . . . . . 164

Figure C.17 Expanded Sample Kinematics . . . . . . . . . . . . . . . . . . . 165

Figure C.18 Expanded Sample Kinematics . . . . . . . . . . . . . . . . . . 166

Figure C.19 Expanded Sample Kinematics . . . . . . . . . . . . . . . . . . . . 167

Figure C.20 Expanded Sample Kinematics . . . . . . . . . . . . . . . . . . . 168

Figure C.21 Expanded Sample Kinematics . . . . . . . . . . . . . . . . . . 169

Figure C.22 Expanded Sample Kinematics . . . . . . . . . . . . . . . . . . . . . 170

Figure C.23 Expanded Sample Kinematics . . . . . . . . . . . . . . . . . . . 171

Figure C.24 Expanded Sample Kinematics . . . . . . . . . . . . . . . . . . . . . . 172

Figure C.25 Expanded Sample Kinematics . . . . . . . . . . . . . . . . . . 173

Figure C.26 Expanded Sample Kinematics . . . . . . . . . . . . . . . . . . . 174

Figure C.27 Expanded Sample Kinematics . . . . . . . . . . . . . . . . . . . . 175

Figure C.28 Expanded Sample Kinematics . . . . . . . . . . . . . . . . . . . 176

Figure C.29 Expanded Sample Kinematics . . . . . . . . . . . . . . . . . . . 177

Figure C.30 Expanded Sample Kinematics . . . . . . . . . . . . . . . . . . . 178

Figure C.31 Expanded Sample Kinematics . . . . . . . . . . . . . . . . . . . 179

Figure C.32 Expanded Sample Kinematics . . . . . . . . . . . . . . . . . . 180

Figure C.33 Expanded Sample Kinematics . . . . . . . . . . . . . . . . . . . . . 181

Figure C.34 Expanded Sample Kinematics . . . . . . . . . . . . . . . . . . . . 182

Figure D.1 Circinus Kinematic Models I . . . . . . . . . . . . . . . . . . . . . 184

Figure D.2 Circinus Kinematic Models II . . . . . . . . . . . . . . . . . 185 
Figure D.3 Mrk 34 Kinematic Models . . . . . . . . . . . . . . . . . . . 186

Figure D.4 Mrk 34 / Mrk 279 Kinematic Models . . . . . . . . . . . . . . . . . 187

Figure D.5 Mrk 1066 / NGC 1667 Kinematic Models . . . . . . . . . . . . . . . . 188

Figure D.6 NGC 3227 Kinematic Models . . . . . . . . . . . . . . . . . . . . . . 189

Figure D.7 NGC 3227 Kinematic Models . . . . . . . . . . . . . . . . . . . . . . 190

Figure D.8 NGC 3227 Kinematic Models . . . . . . . . . . . . . . . . . . . . . 191

Figure D.9 NGC 3783 / NGC 4051 Kinematic Models . . . . . . . . . . . . . . . 192

Figure D.10 NGC 4051 / NGC 4507 Kinematic Models . . . . . . . . . . . . . . 193

Figure D.11 NGC 5506 / NGC 5643 Kinematic Models . . . . . . . . . . . . . . 194

Figure D.12 NGC 5643/ NGC 7674 Kinematic Models . . . . . . . . . . . . . . 195 


\section{LIST OF ABBREVIATIONS}

\begin{tabular}{|c|c|}
\hline AGN & Active Galactic Nuclei \\
\hline BLR & broad-line region \\
\hline BLS1 & broad-line Seyfert 1 galaxy \\
\hline CCD & Charged Coupled Device \\
\hline ENLR & extended narrow-line region \\
\hline FOC & Faint Object Camera \\
\hline FWHM & full-width at half-maximum \\
\hline $\mathbf{H}_{2} \mathbf{0}$ & Water \\
\hline$H S T$ & Hubble Space Telescope \\
\hline IC & Index Catalogue \\
\hline IDL & Interactive Data Language \\
\hline IR & infrared \\
\hline IRAS & Infrared Astronomical Satellite \\
\hline LOS & line-of-sight \\
\hline MAST & Multimission Archive at Space Telescope \\
\hline $\mathrm{MCG}$ & Morphological Catalog of Galaxies \\
\hline Mrk & Markarian \\
\hline NASA & National Aeronautic and Space Administration \\
\hline NGC & New General Catalogue \\
\hline NLR & narrow-line region \\
\hline NLS1 & narrow-line Seyfert 1 galaxy \\
\hline pc & parsecs \\
\hline SED & spectral energy distribution \\
\hline SMBH & supermassive black hole \\
\hline
\end{tabular}


STIS

UV

$\mathrm{WF} / \mathrm{PC}$

WFPC2

WFC3

3CR

$3 \mathrm{C}$

3D
Space Telescope Imaging Spectrograph

ultraviolet

Wide Field Planetary Camera 1

Wide Field Planetary Camera 2

Wide Field Camera 3

Third Cambridge Revised Catalog

Third Cambridge Catalog

three dimensional 
CHAPTER 1

\section{INTRODUCTION}

Within all massive galaxies in the universe are supermassive black holes (SMBHs) which reside in the center of their hosts and whose masses exceed $10^{6}$ solar masses $\left(\mathrm{M}_{\odot}\right)$. While most galaxies in the current epoch contain SMBHs that lie dormant, a small percentage of galaxies known as Active Galactic Nuclei (AGN) contain SMBHs at their center that are actively gaining mass from the surrounding matter of an accretion disk. As the matter falls into the SMBH, it loses angular momentum and gravitational potential energy and as a result emits a massive amount of electromagnetic radiation, often outshining the rest of the galaxy.

Edward A. Fath was the first to obtain an optical spectrum of an active galaxy when he noted the presence of strong emission lines in the "spiral nebula" NGC 1068. Soon after, V.M. Slipher obtained a higher-resolution spectrum of NGC 1068, making the observation that the emission lines seen by Fath were resolved and had widths of hundreds of kilometers per second. Carl K. Seyfert (1943) was the first to realize that NGC 1068 and several other high-excitation emission line galaxies, including NGC 3516, NGC 4051, NGC 4151, and NGC 7469, were in fact quite similar and formed a distinct class of objects that contained broad permitted emission lines (up to $8500 \mathrm{~km} \mathrm{~s}^{-1}$, full width at zero intensity), with the hydrogen lines sometimes broader than the other lines.

This group of galaxies has since been defined as Seyfert galaxies, which in turn are a relatively low luminosity $\left(L_{b o l} \approx 10^{43}-10^{45} \operatorname{erg~s}^{-1}\right)$, nearby $(\mathrm{z} \leq 0.1)$ subset of the overall collection of AGN. The dichotomy of broadened and unbroadened hydrogen and other permitted emission lines has also led the Seyfert class of objects to be divided into two groups (Khachikian \& Weedman 1974). Seyfert 1 galaxies were defined to have spectra containing broad (full width at half-maximum $[\mathrm{FWHM}] \geq 1000 \mathrm{~km} \mathrm{~s}^{-1}$ ) permitted lines, narrower $\left(\mathrm{FWHM} \leq 1000 \mathrm{~km} \mathrm{~s}^{-1}\right)$ forbidden lines, and distinct, nonstellar optical and UV 
continua. Seyfert $2 \mathrm{~s}$ differed in that they contained only narrow permitted and forbidden lines, and their optical and UV continua were dominated by the host galaxy.

\subsection{Seyfert Galaxy Components}

Understanding the morphology of a Seyfert galaxy is integral to this study and thus we must define its components early on. Moving from the inside out, the very gravitational center of the galaxy contains a SMBH. Fueling the SMBH is the surrounding accretion disk, which is also the source of the intense continuum radiation characteristic of AGN. Near the accretion disk is the broad-line region (BLR), the source of broad lines visible in Seyfert 1 galaxies. Only permitted and some semi-forbidden lines have been observed to be broadened, indicating that this region must be close to the nucleus where high densities can collisionally de-excite the low-transition probability states that form the forbidden lines. Indeed, the BLR is a region of dense $\left(\mathrm{n}_{H}=10^{8}-10^{12} \mathrm{~cm}^{-3}\right)$ ionized clouds of gas a few light days in diameter (Peterson et al. 2004). Outside the BLR is the NLR, a knotty, extended (1 - 1000 pc) region of ionized clouds sufficiently less dense than the BLR $\left(\mathrm{n}_{H} \leq 10^{6} \mathrm{~cm}^{-3}\right)$ that emits the narrow, permitted and forbidden, lower-velocity emission lines seen in the optical and UV. The NLRs of Seyfert galaxies contain gas that has been photoionized by the nonstellar continuum emission of the AGN and are roughly biconical in structure, with the apex of the bicone residing in the central AGN (Pogge 1988; Schmitt et al. 1994). This observation suggests that the ionizing radiation is collimated by an optically thick absorbing material, which is likely a 'torus-like' obstruction (Antonucci \& Miller 1985) or possibly a disk wind (Elitzur \& Shlosman 2006), at small radial distances of a few parsecs. Around the same scale and position of the NLR, Seyferts also sometimes contain radio emitting knots of low-density plasma, which suggests that there may be some connection between the thermal narrow-line gas and the non-thermal plasma. At radial distances of $\geq 1 \mathrm{kpc}$, ionized gas often exists in an extended NLR (ENLR) likely in the plane of the host galaxy (Unger et al. 1987). Seyfert 


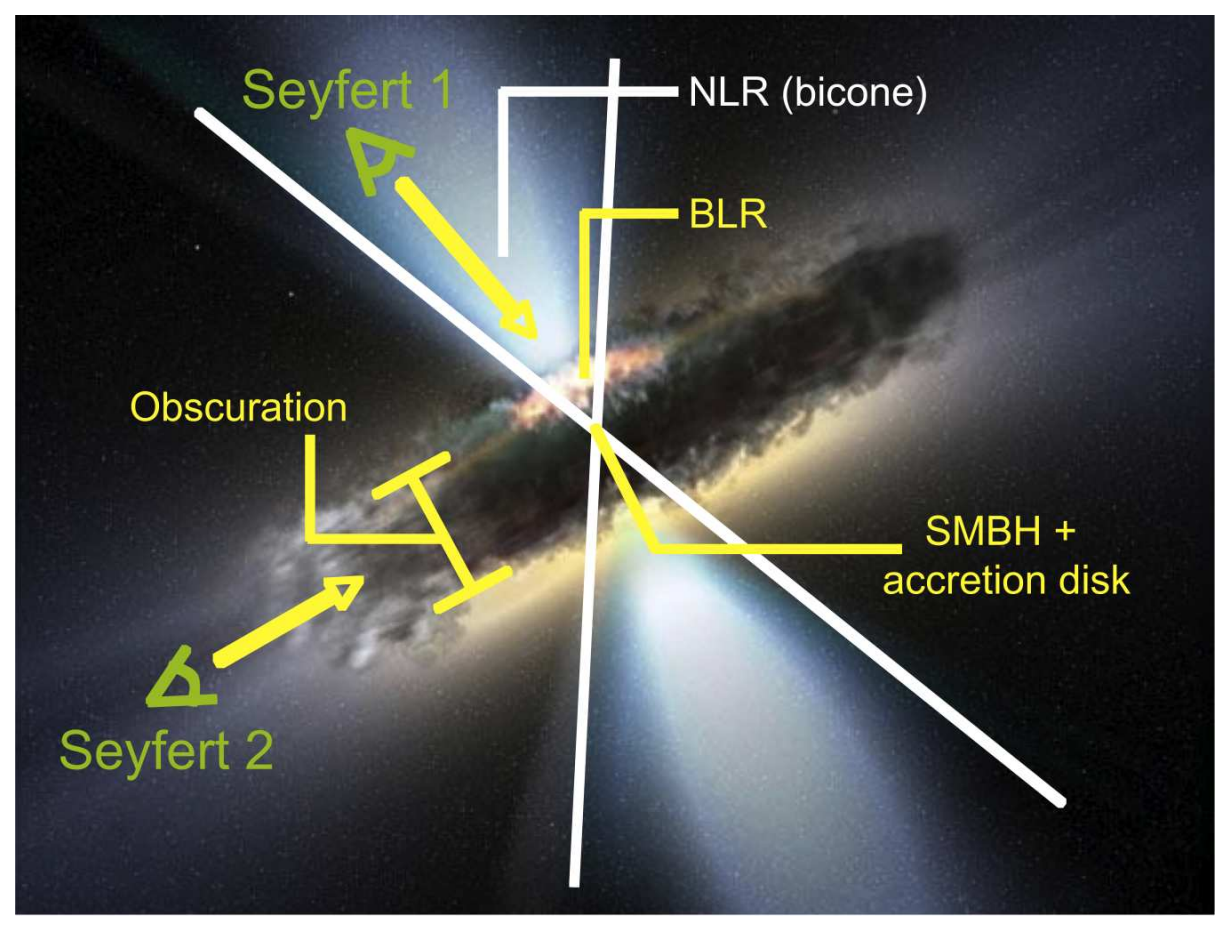

Figure 1.1 Picture of an AGN showing the major components.

hosts can be any species of galaxy but are most often spirals. Figure 1.1 shows a cartoon depicting the various described AGN components.

\subsection{The Unified Model}

If the general anatomy of AGN is similar, the question arises as to why are there two types of Seyfert galaxies? Osterbrock (1978) suggested that Seyfert 1s and 2s were physically the same objects, but that the BLRs of Seyfert 2s are obscured from our view by dust surrounding the central engine. Thus, an observer looking down the torus axis of an AGN would see the central source, the BLR, and the NLR, and thus see the spectrum of a Seyfert 1. Alternatively, an observer looking at the same AGN from the plane of the "torus" would only see the NLR and a small amount of emission from the nuclear source, detecting a Seyfert 2. The strongest observational evidence for a unified model stems from work by Antonucci \& Miller (1985), who discovered that weak, polarized broad emission lines exist in many 
Seyfert 2 AGN by analyzing the polarized spectrum of the Seyfert 2 NGC 1068. These broad emission lines are likely due to reflection of the nuclear light by dust or electrons along our line of sight (LOS). Additionally, the continuum fluxes observed for Seyfert 2s do not appear to be large enough to produce their corresponding observed emission line fluxes (Kinney et al. 1991), indicating the continuum source is obstructed in these galaxies. As Seyfert 1s show the required level of radiation, this finding also indicates an orientation effect. In the mid-infrared (mid-IR), strong continuum fluxes originating from hot dust near the centers of Seyfert 2s also support the presence of dusty obscuration in front of the continuum source (Storchi-Bergmann et al. 1992). Finally, narrow-band images of Seyfert NLRs often show biconical structures, suggesting they are formed via collimation by a surrounding, torus-like obscuration (Schmitt \& Kinney 1996).

\subsection{The Narrow-Line Region}

For this work, we focus our studies on the NLRs in Seyfert galaxies, as they are the only AGN component that can be spatially resolved in the optical, with the Hubble Space Telescope $(H S T)$. Morphologically, these regions are generally axisymmetric rather than spherically symmetric and, again, often extend in the same general direction as the radio jet axes when the latter are detected. The majority of the NLR gas is photoionized and often driven in outflow by the central engine (Crenshaw et al. 2000; Crenshaw \& Kraemer 2000). Recent studies have shown that most of the emission lines seen in the NLR can be accounted for by assuming a central ionization source illuminating a multicomponent gas characterized by different densities (Kraemer \& Crenshaw 2000a; Kraemer et al. 2000). In addition to the success of photoionization models, misalignment of the radio jet and NLR in NGC 4151, as shown by Mundell et al. (2003), supports photoionization of the NLR rather than collisional ionization by the jet as the dominant source of ionization. AGN photoionization through the openings of an optically thick torus is then the source of the striking biconical NLR 
morphology seen in some AGN. Observed primarily in Seyfert 2s, these biconical formations have projected opening angles typically in the range of $\sim 30^{\circ}-100^{\circ}$ with well defined linear edges that imply the NLR is defined by collimation of light from the nuclear source. The large [O III] $\lambda 5007$ / H $\beta$ flux ratio inside the conical NLR is characteristic of low-density gas ionized by an AGN continuum, while outside the cone the ratio is lower, indicating that this gas is primarily ionized by starlight instead of an AGN (Peterson 1997).

\subsection{Outflows in the NLR}

We have previously shown that the NLR kinematics in Seyfert galaxies are often dominated by radial outflow in the approximate shape of a bicone, through kinematic modeling of the NLRs of NGC 1068 (Seyfert 2), NGC 4151 (Seyfert 1), and Mrk 3 (Seyfert 2) (Crenshaw et al. 2000; Crenshaw \& Kraemer 2000; Ruiz et al. 2001; Das et al. 2005, 2006; Crenshaw et al. 2010a).

Our current outflow model originated in the Crenshaw \& Kraemer (2000) study while attempting to determine the nature of the NLR kinematics as possibly due to infall, rotation, outflow, or some other flow pattern. By observing NGC 1068, the nearest bright Seyfert 2 galaxy, with the HST Space Telescope Imaging Spectrograph (STIS), they were able to resolve [O III] emission-line knots that presumably accelerated out from the inner nucleus, reached a terminal velocity, and then decelerated due to drag through interactions with the surrounding ambient material. Additionally, a lack of low radial velocities where the kinematic curves peaked suggested that the NLR was evacuated of [OIII] emission along its axis, which is close to the plane of the sky in NGC 1068. These data, combined with HST imaging that indicated a biconical geometry for the NLRs of many Seyfert 2 galaxies (Schmitt \& Kinney 1996), led to the postulation that a radial outflow shaped as a biconical shell could reproduce the NLR kinematics seen in the Seyfert 2 galaxy NGC 1068. The resultant kinematic model assumes the knots are radially outflowing and accounted for by 
a simple velocity law close to the nucleus, $v=k r$, where $r$ is the distance from the nucleus and $k$ is a constant. In addition to gaining a better understanding of the NLR kinematics, their model also produced a geometry of the NLR, including an inclination of $5^{\circ}$ out of the plane of the sky, which was required for the model to fit the observed radial velocities.

Crenshaw et al. (2000) proceeded to apply their new kinematic model to the NLR of the brightest Seyfert 1 galaxy NGC 4151, in which they found, similarly to their model of NGC 1068, evidence for radial acceleration and subsequent deceleration of emission-line knots and a hollowed region near the axis. The largest difference between the two NLR models was their inclination from our line of sight. The bicone axis of NGC 4151 was inclined closer to our line of sight at $\sim 45^{\circ}$ out of the plane of the sky versus $\sim 5^{\circ}$ out of the plane of the sky, nearly perpendicular to our line of sight, for NGC 1068. By matching the observed radial velocities of a second Seyfert galaxy to their kinematic model, it was realized that it could be possible to determine not just the nature of the NLR kinematics, but the orientation and geometry of the AGN system as well.

Das et al. $(2005,2006)$ tested the outflow model with more detailed data sets using higher-resolution spectra at multiple slit locations across the NLRs of NGC 4151 and NGC 1068 respectively. The original modeling code used by Crenshaw \& Kraemer (2000) was also updated to include a variable $n$ in the velocity law, $v=k r^{n}$. They concluded that the models from Crenshaw et al. (2000) and Crenshaw \& Kraemer (2000) were consistant with their own and that the original velocity law remained the strongest fit to the kinematic data.

The last AGN outflow modeled prior to our current study was that of Mrk 3, where Ruiz et al. (2001) employed a combination of STIS medium resolution slitless and low resolution long slit spectra to produce a similar kinematic model to the previous two Seyferts. The geometry of their published model did not include significant knots of emission seen in imaging and thus Crenshaw et al. (2010a) revisited Mrk 3 to develop a new model that included a larger opening angle to account for the previously avoided offset emission knots, 
and an inner gas/dust disk was adopted, which originated from tidally drawn material of the gas disk in neighboring spiral galaxy UGC 3422, to explain the ENLR emission seen in imaging.

\subsection{Motivation for the Current Study}

While there is some evidence that Seyfert 1 AGN are viewed more pole-on and Seyfert 2 AGN are viewed more edge-on with respect to the obscuring toroidal structure, the specific inclinations of individual AGN are generally unknown. Thus, after decades of study, we still do not know how the properties of AGN change with inclination further than comparing Seyfert 1s and 2s. By employing our NLR kinematics mapping technique, we can for the first time determine our viewing angle to a sample of AGN and begin finding inclination dependancies, allowing us to progress beyond the simple unified model of Type 1 and 2 AGN.

In order to determine if any correlations exist between inclination and other AGN parameter, we require more data points than the three individual AGN previously modeled by our group. Thus, the motivation behind this study is to determine the inclinations of a larger sample of individual AGN and compare the determined inclinations from all works with observed physical parameters of the AGN to identify any existing correlations. Chapter 2 describes the observations used in our work and a description of the analysis and modeling is given in Chapter 3. The first target modeled in this work, Mrk 573, is described at length to further explain our process in Chapter 4. Chapter 5 applies our biconical outflow model to Mrk 78 in order to determine if targets that exhibit dual sets of narrow emission lines can be shown to originate from a single AGN source. Chapter 6 expands our study in attempt to determine inclinations for all Seyfert AGN with optical NLR observations from STIS, and Chapter 7 then compares inclinations of every AGN modeled by our group to observable AGN parameters before conclusions are made in Chapter 8. 
CHAPTER 2

OBSERVATIONS AND ANALYSIS

\subsection{HST STIS}

All spectral observations for this work were taken with HST STIS, with the charged-couple device $(\mathrm{CCD})$ through either a slit of $52^{\prime \prime} \times 0.2^{\prime \prime}$, or $52^{\prime \prime} \times 0.1^{\prime \prime}$, or in slitless mode (Section 6.1.3). Spectra were dispersed into spectral images (Figure 2.1) to observe [O III] $\lambda 5007$ and $\mathrm{H} \alpha \lambda 6563$ emission. Specifications of each grating used are listed in Table 2.1.

For our measurements, we used the [O III] $\lambda 5007$ and $\mathrm{H} \alpha$ emission lines as they are the strongest optical lines originating in the NLR ([O III $] / \mathrm{H} \beta \approx 10 ; \mathrm{H} \alpha / \mathrm{H} \beta \approx 3$; Peterson (1997)). The STIS image scale is $0.05^{\prime \prime} \mathrm{pixel}^{-1}$ and the spatial resolution is $0.1^{\prime \prime} \mathrm{pixel}^{-1}$ in the cross-dispersion direction.

The STIS spectra were processed using Interactive Data Language (IDL) software developed at NASA's Goddard Space Flight Center for the STIS Instrument Definition Team. Cosmic-ray hits were removed before further processing. The zero points of the wavelength scales were corrected using wavelength-calibration exposures taken after each observation. Finally, the spectra were geometrically rectified and flux calibrated to provide spectral images that have a constant wavelength along each column and display fluxes in units of erg s ${ }^{-1}$ $\mathrm{cm}^{-2} \AA^{-1}$ per cross-dispersion pixel. Occasionally, a number of hot pixels remained after the data were processed. In those cases, we performed an additional cleaning step by replacing

Table $2.1 H S T /$ STIS grating specifications

\begin{tabular}{ccccc}
\hline Grating & $\begin{array}{c}\text { Spectral Range } \\
(\AA)\end{array}$ & $\begin{array}{c}\text { Target } \\
\text { Line }\end{array}$ & $\begin{array}{c}\text { Resolving Power } \\
(\lambda / \Delta \lambda)\end{array}$ & $\begin{array}{c}\text { Resolving Power } \\
\left(\mathrm{km} \mathrm{s}^{-1} \text { FWHM }\right)\end{array}$ \\
\hline G430L & $2900-5700$ & {$[\mathrm{O}$ III] $\lambda 5007$} & $\sim 900$ & $\sim 330$ \\
G430M & $3020-5610$ & {$[\mathrm{O}$ III $] \lambda 5007$} & $\sim 9000$ & $\sim 30$ \\
G750M & $5450-10,140$ & H $\alpha \lambda 6563$ & $\sim 5900$ & $\sim 50$ \\
\hline
\end{tabular}




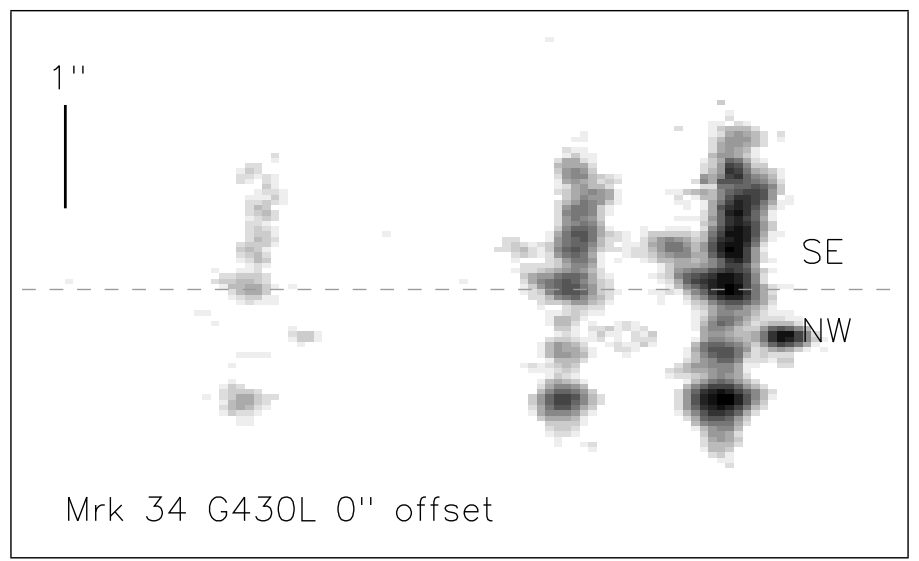

Figure 2.1 HST/STIS long-slit spectral image of Mrk 34. Dashed line gives the location of the kinematic center. Wavelength increases to the right, with optical lines $\mathrm{H} \beta \lambda 4861$ and [O III] $\lambda \lambda 4959,5007$ from left to right respectively. The knotty structure of the NLR emission-line gas is apparent.

the bad pixel with a local median value in the data.

\subsection{Line Analysis}

Observations in each slit were analyzed in the same manner for all galaxies. The analysis below explains the procedure we used to extract velocities and other information from a long-slit observation. Thus, the same procedure applies to every slit position in our sample (excluding slitless observations). Each spectral image produced one spectrum per crossdispersed pixel along the slit centered on the [OIII] or $\mathrm{H} \alpha$ emission lines. Because there are 2 pixels per resolution element, the data are slightly oversampled. In each spectrum, individual components of [OIII] $\lambda 5007$ or $\mathrm{H} \alpha \lambda 6563$ emission lines were fit with Gaussians over an average continuum taken from line-free regions throughout the spectrum. Many spectra contained two or more kinematic components, where each identifiable peak was fit with a Gaussian. We employ a Gaussian fit rather than a direct integration across the line profile because in most cases the former is more suited to extract individual velocities from blended kinematic components. Noisy spectra $(\mathrm{S} / \mathrm{N}<3$ per resolution element) or broad 
$\mathrm{H} \alpha$ lines near the nucleus of Seyfert 1s, which blended into the surrounding [NII] doublet, were not fitted.

Figure 2.2 depicts a typical progression of spectra illustrating the fitting of multiple components with change in position. The graphs represent spectra taken from the central slit position over Mrk 34 and range from $0^{\prime \prime}$ to $0.3^{\prime \prime}$ in increments of $0.05^{\prime \prime}$, stepping away from the nucleus in the eastern direction.

In the initial figure, a central kinematic component is visible, which we fit with a Gaussian. As we step away from the nucleus, a second blueshifted kinematic component emerges to the left of the original component and is also fit with a Gaussian. A possible third component is visible in the fourth and fifth panels which is ultimately fit with a Gaussian as it also provides a better fit to the adjacent components. The appearance of the same components in multiple rows along the slit indicates that we are obtaining multiple measurements of spatially resolved knots of emission.

\subsection{Calculating Velocities}

The central peak of each Gaussian is the central wavelength from which we measured a Doppler shifted velocity for both the [O III] $\lambda 5007$ and $\mathrm{H} \alpha$ emission lines. The radial velocities in the rest frame of the galaxy are found as follows:

$$
v_{r}=\left(\frac{\lambda_{o b s}}{\lambda_{l a b}}-z-1\right) \times c
$$

where $\lambda_{o b s}$ is the observed central wavelength measured from the Gaussian fit, $\lambda_{l a b}$ is the vacuum rest wavelength of the [O III] or $\mathrm{H} \alpha$ emission at $5008.2 \AA$ and $6564.6 \AA$ respectively, $z$ is the systemic redshift of the galaxy, and $c$ is $2.998 \times 10^{5} \mathrm{~km} \mathrm{~s}^{-1}$, the speed of light in a

vacuum. In solving for $v_{r}$, we do not need the relativistic formula for velocity because our velocities,$v_{r}$, are always less than $2000 \mathrm{~km} \mathrm{~s}^{-1}$. 

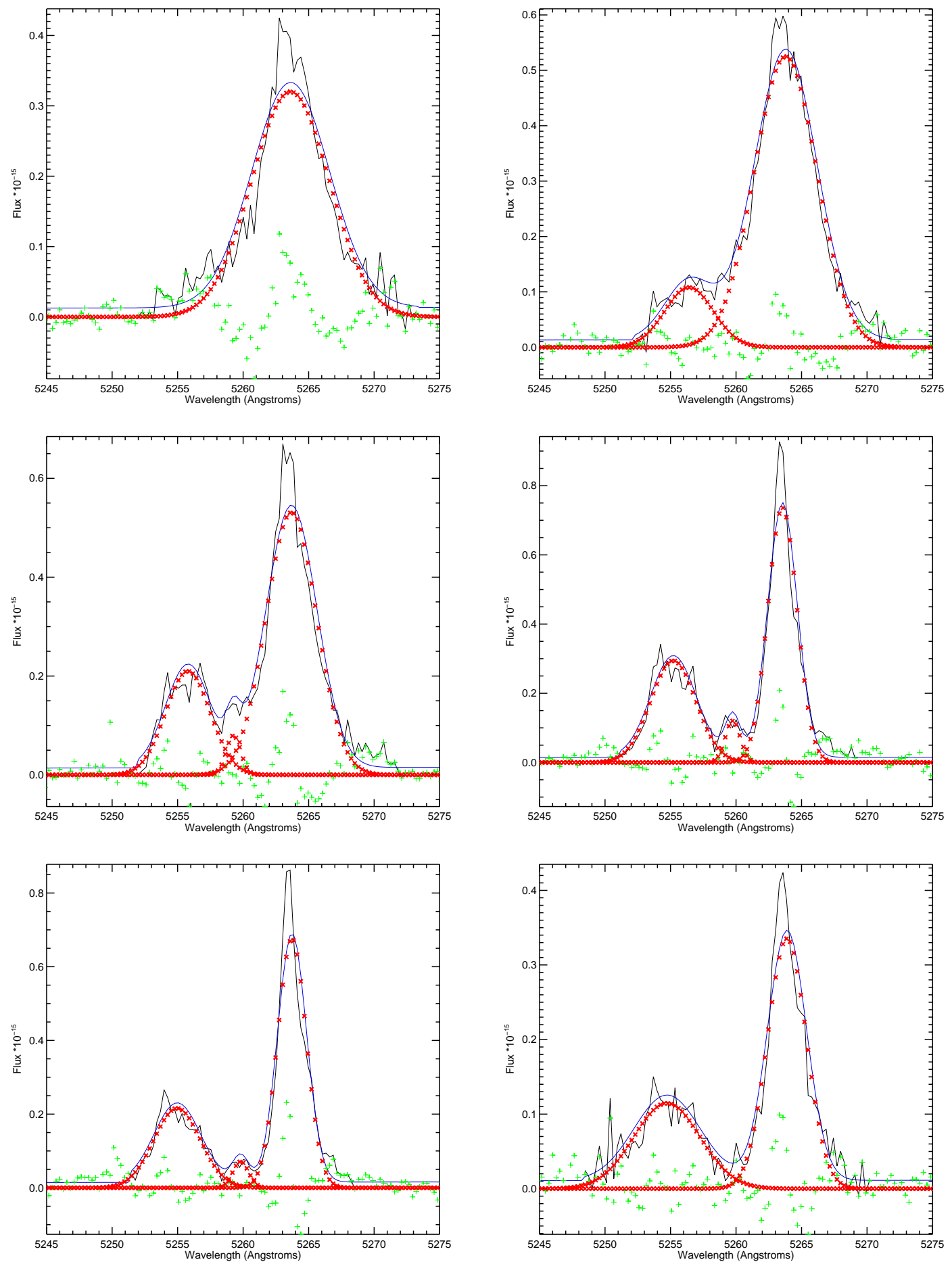

Figure 2.2 Spectra in black and fits in color showing the multicomponent Gaussian fits. The plots, ordered from left to right and top to bottom, are from positions separated by $0.05^{\prime \prime}$, increasing in distance from the nucleus. Red $\times$ curves represent the fit for each component, blue curves represent the sum of the red curves, and green + curves represent the difference between the black spectra and red fitted curves. Spectra are not yet Doppler-corrected for the cosmological redshift of Mrk 34. Fluxes are in ergs s${ }^{-1} \mathrm{~cm}^{-2} \AA^{-1}$ 


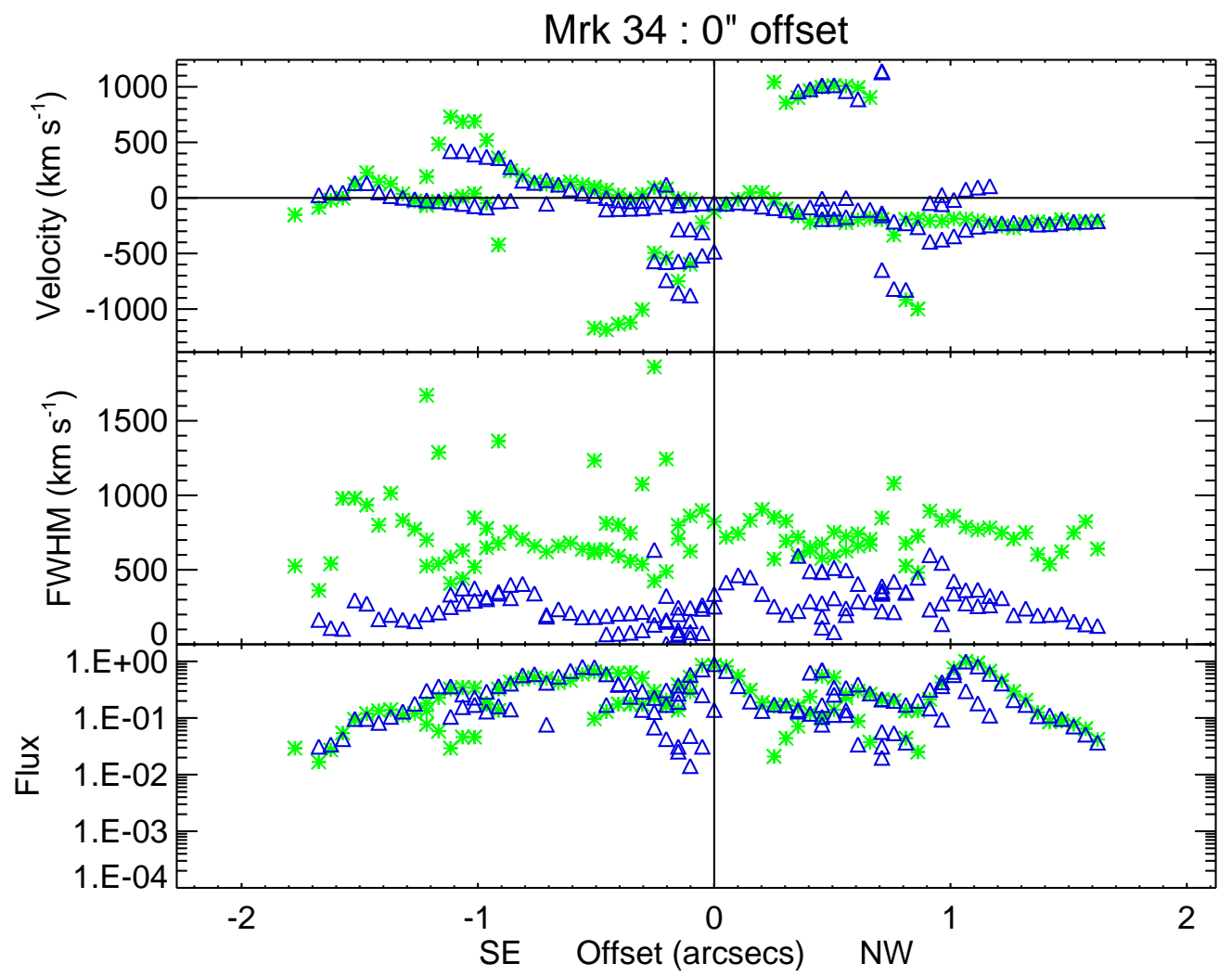

Figure 2.3 Radial velocites (top), FWHM (middle), and normalized fluxes (bottom) of [O III] lines using the G430L (green stars) and G430M (blue triangles) gratings for the central slit position of Mrk 34 .

Figure 2.3 shows the entire velocity data set, as well as full-width at half-maxima (FWHM), and normalized fluxes across the central slit position for Mrk 34. Note that multiple components exist at several positions along the spectral image containing velocities that generally agree between the two gratings. Discrepancies between the FWHMs of the two gratings are due to different resolutions of the gratings. Adding the FWHM of the resolved $\mathrm{H} \alpha$ lines to the FWHM of the line spread function of the G430L grating for a $0.2^{\prime \prime}$ slit in quadrature gives a value typical of the observed FWHM of the [O III] lines. Lumpiness of fluxes across the long slit observation are due to spatially resolved knots of emission, as seen in Figure 2.1. 


\subsection{Uncertainties}

There are three sources of uncertainty in our velocity measurements. The first is that the measured emission lines are not perfect Gaussians, as shown in Figure 2.2. To measure a typical value for this uncertainty, denoted as $\sigma_{h i}$, we found the average difference between Gaussians fit to a selection of 15 random, isolated high signal-to-noise emission lines as detailed in Section 2.2 and the actual centroid of the same line using the IDL feature.pro program, an analysis tool which measures essential attributes (i.e. central wavelength, total flux, etc.) of a spectral feature instead of its Gaussian fit. The second error comes from photon noise. Errors due to noisy spectra were measured using the assumption:

$$
\sigma_{l o}^{2}=\sigma_{h i}^{2}+\sigma_{\text {noise }}^{2},
$$

where the variance in wavelength when fitting a Gaussian to a low signal-to-noise line is due to a combination of the imperfect Gaussian fit $\left(\sigma_{h i}\right)$ and noise in the data $\left(\sigma_{\text {noise }}\right)$. By applying similar measurements performed on high S/N lines to 15 random, low S/N lines and subtracting in quadrature the subsequent averaged value from the average $\sigma_{h i}$, we can solve for $\sigma_{\text {noise }}$. The third error is the standard deviation $\left(\sigma_{d e v}\right)$ in the velocities of an individual knot of emission that extends along the slit. All three errors are given for each grating in Table 2.2 , as well as the total maximum error $\left(\sigma_{\text {total }}\right)$, where

$$
\sigma_{\text {total }}^{2}=\sigma_{h i}^{2}+\sigma_{\text {noise }}^{2}+\sigma_{\text {dev }}^{2}
$$

In general, the most significant errors are attributed to velocity deviation across individual clouds. Varied continuum placements for randomly selected spectra were also tested, but did not affect the central wavelength significantly.

From Table 2.2, it can be seen that the dominant uncertainty stems from $\sigma_{d e v}$. This error often represents a velocity gradient across a knot of emission, as shown in later sections, but remains a valid estimate of uncertainty in the radial velocity centroid of the total knot. It 
Table 2.2 Maximum errors in velocity measurements

\begin{tabular}{cccccc}
\hline Grating & $\begin{array}{c}\sigma_{\text {hi }} \\
(\AA)\end{array}$ & $\begin{array}{c}\sigma_{\text {noise }} \\
(\AA)\end{array}$ & $\begin{array}{c}\sigma_{\text {dev }} \\
(\AA)\end{array}$ & $\begin{array}{c}\sigma_{\text {total }} \\
(\AA)\end{array}$ & $\begin{array}{c}\sigma_{\text {total }} \\
\left(\mathrm{km} \mathrm{s}^{-1}\right)\end{array}$ \\
\hline G430L & .26 & .79 & 1.74 & 1.93 & 115.5 \\
G430M & .11 & .13 & 1.71 & 1.72 & 102.9 \\
G750M & .07 & .34 & 0.57 & 0.67 & 40.1 \\
\hline
\end{tabular}

should be noted that $\sigma_{\text {total }}$ is an upper limit that accounts for the maximum value of each contributing error. For example, the total error will be lower for high signal-to-noise spectra as $\sigma_{\text {noise }}$ will be close to zero. Understanding the possible range of errors is important for determining a best fit of our kinematic model to the spectral data and the model parameter uncertanties. 
CHAPTER 3

\section{KINEMATIC MODEL}

\subsection{General Assumptions}

The kinematic models generated to match the observations are simple, yet give good fits to radial velocities for both Seyfert 1s and 2s. This simple approach stems from four basic assumptions concerning the characteristics of the NLR clouds:

1) The model employs a biconical geometry for the NLR, with both cones being identical. This geometry best explains how [O III] images often show axisymmetric, triangular NLRs for Seyfert 2s and compact circular or elliptical NLRs for Seyfert 1s and agrees with the unified model (Schmitt et al. 2003).

2) The model assumes that the biconical geometry is due to the illumination from the nucleus surrounded by a torus-like structure of optically thick material. Thus, the apex of the bicone originates at the nucleus of the AGN (i.e. the SMBH).

3) The model assumes a filling factor of 1 within the hollow bicone geometry and 0 outside, as we do not know the location of a cloud along our LOS through the bicone walls. This assumption gives a range of velocites along any LOS that a cloud can occupy. We assume that there is no absorption of this line within the bicone by the gas because the majority of our analysis is based on a forbidden line, although dust may actually absorb some of this emission.

4) The model assumes that the bicone edges are sharp. Thus, the inner and outer opening angles define edges of ionized gas. However, in reality, the observed bicone often has fuzzy edges (Kraemer \& Crenshaw 2000b), but this has little effect on our results, as discussed later. This also applies to the ends of the bicone defined by its total length, so that the model does not apply to clouds at greater distances. 
Table 3.1 Parameter Abbreviations

\begin{tabular}{lc}
\hline Parameter & Symbol \\
\hline Position angle of bicone axis $\left(^{\circ}\right)$ & $P . A$. \\
Inclination of bicone axis $\left(^{\circ}\right)$ & $i$ \\
Outer opening angle $\left(^{\circ}\right)$ & $\theta_{\max }$ \\
Inner opening angle $\left(^{\circ}\right)$ & $\theta_{\min }$ \\
Maximum velocity $\left(\mathrm{km} \mathrm{s}^{-1}\right)$ & $v_{\max }$ \\
Bicone height $(\mathrm{pc})$ & $z_{\max }$ \\
Turnover radius $(\mathrm{pc})$ & $r_{t}$ \\
\hline
\end{tabular}

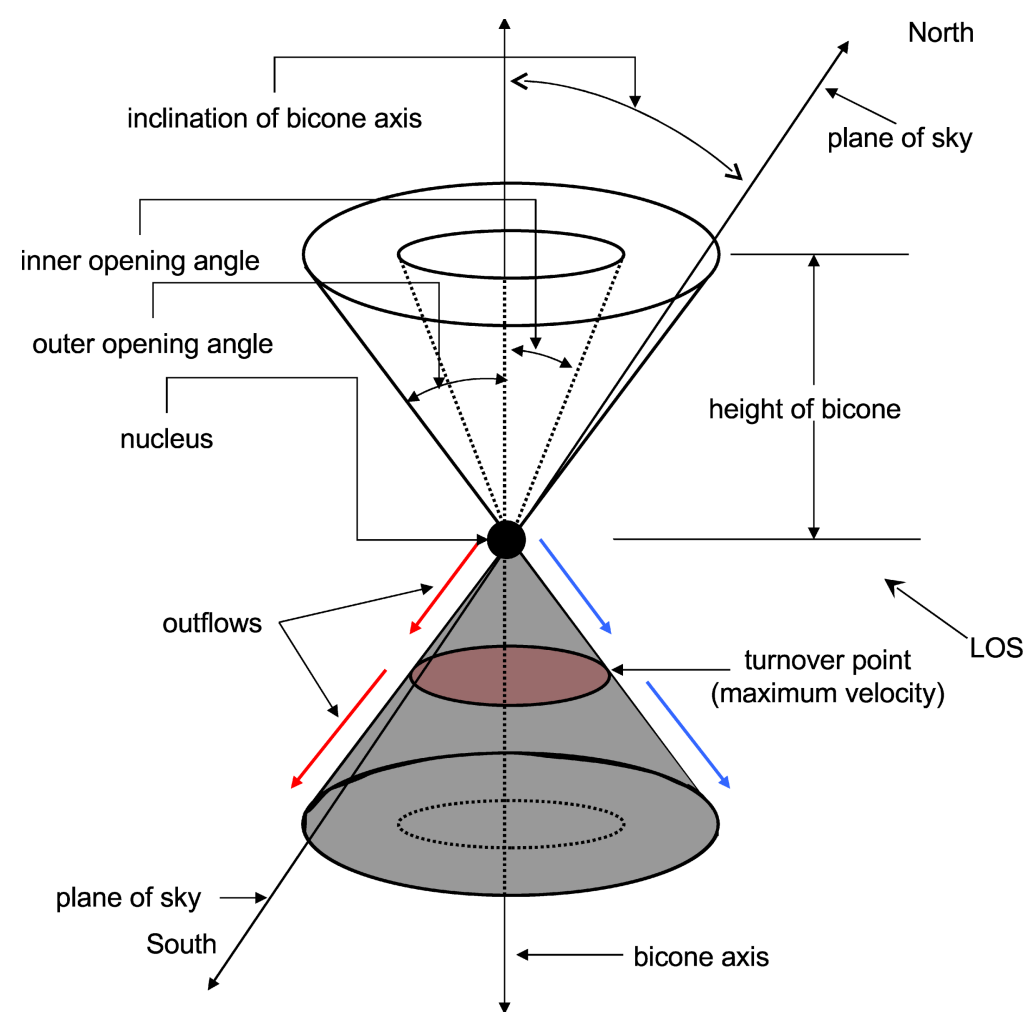

Figure 3.1 Figure displaying all alterable parameters used to create a kinematic model.

\subsection{Model Parameters}

The kinematic models used in this study are generated in a 3-dimensional geometry that depends on some basic input parameters. These parameters are listed in Table 3.1, and the cartoon in Figure 3.1 is used as reference (Das et al. 2005). 
The position angle is the angle between North and the bicone axis in the plane of the sky, measured in the eastward (counter-clockwise) direction. The inclination of the bicone is measured out of the plane of the sky. The inner and outer opening angles are given as half of the total opening angles, measured from the bicone axis to the given opening angle. The maximum velocity is the boundary value set in the velocity law $v=k r$, where the kinematics accelerate out (as a result of radiation pressure, for example) to a distance where the maximum velocity is reached before deceleration occurs. The maximum height is the distance from the nucleus to one end of the bicone, measured along the bicone axis. The turnover radius is the specific distance where clouds are no longer accelerated and begin to decelerate back to systemic velocity (due to, for example, gravitational or drag forces).

\subsection{Creating the Model}

As mentioned in Section 1.4, the kinematic model used in this work originated in the Crenshaw \& Kraemer (2000) study where the code generated a two-dimensional velocity map and sampled the map with a slit that matched the position, orientation, and width of an observed slit. The kinematic modeling code has since been updated (Das et al. 2005) to produce a three-dimensional velocity cube which is sampled by extracting a two-dimensional sub-array along the position, orientation, and width of an observed slit that contains all the radial velocity values within that slit. This model allows for different velocity laws of the form $v=k r^{n}$ at $r \leq r_{t}$ (turnover radius) and $v=v_{\max }-k r^{n}$ at $r>r_{t}$. We found that the linear form was sufficient as the resultant models fit the data better than the other laws in general.

Figure 3.2 shows a front and back to a generic model velocity cube in a similar orientation as the toy model in Figure 3.1 as viewed from Earth. Blue colors represent blueshifted velocities and red colors represent redshifted velocities. Next to the three-dimensional velocity

model are two-dimensional velocity extractions, radial velocities extracted along the slit and 
plotted as a function of position in arcseconds, which are eventually fit to our kinematic data. The extraction consists of four 'components', two from the front side of the bicone and two from the back side. The upper two graphs highlight velocities extracted from the top and bottom components, respectively, of the front side of the bicone. Likewise, the lower two graphs highlight velocities extracted from components of the back of the bicone. Radial velocities are extracted along the slit and plotted as a function of position in arcseconds.

\subsection{Fitting the Model to Data}

Model parameters are initially set to observed values, taken from imaging $\left(z_{\max }, \theta_{\max }\right.$, P.A.) and the kinematics data $\left(v_{\max }, r_{t}\right)$. By creating a model parameter set and extracting slit positions corresponding to all observational positions for a particular galaxy, we can compare the two-dimensional model velocity extractions to the data simultaneously (Figure 2.3). The comparison is done by eye as we have found no effecient statistical algorithm to compare the model and data. For example, expanding the width of the cone $\left(\theta_{\max }-\theta_{\min }\right)$ will always include more observed velocities and result in a reduced $\chi^{2}$, but more empty space in the kinematic plot is also included. The process of model fitting is as follows:

1) The best fit model parameters are obtained when a model encloses the maximum amount of data points within a minimum shaded region (i.e. minimum difference between $\theta_{\min }$ and $\left.\theta_{\max }\right)$ and also matches the trend of increasing and decreasing velocity in the data reasonably well.

2) Input parameters cannot change across individual slits, as models must be consistant for all slits for each galaxy.

3) The shaded regions in the models should not necessarily contain all data points, because some points are likely not in the NLR (e.g. they may not be in the host galaxy) and thusly are not incorporated in the bicone geometry. Emphasis is placed on the higher flux velocities, which generally followed a more structured curve. 

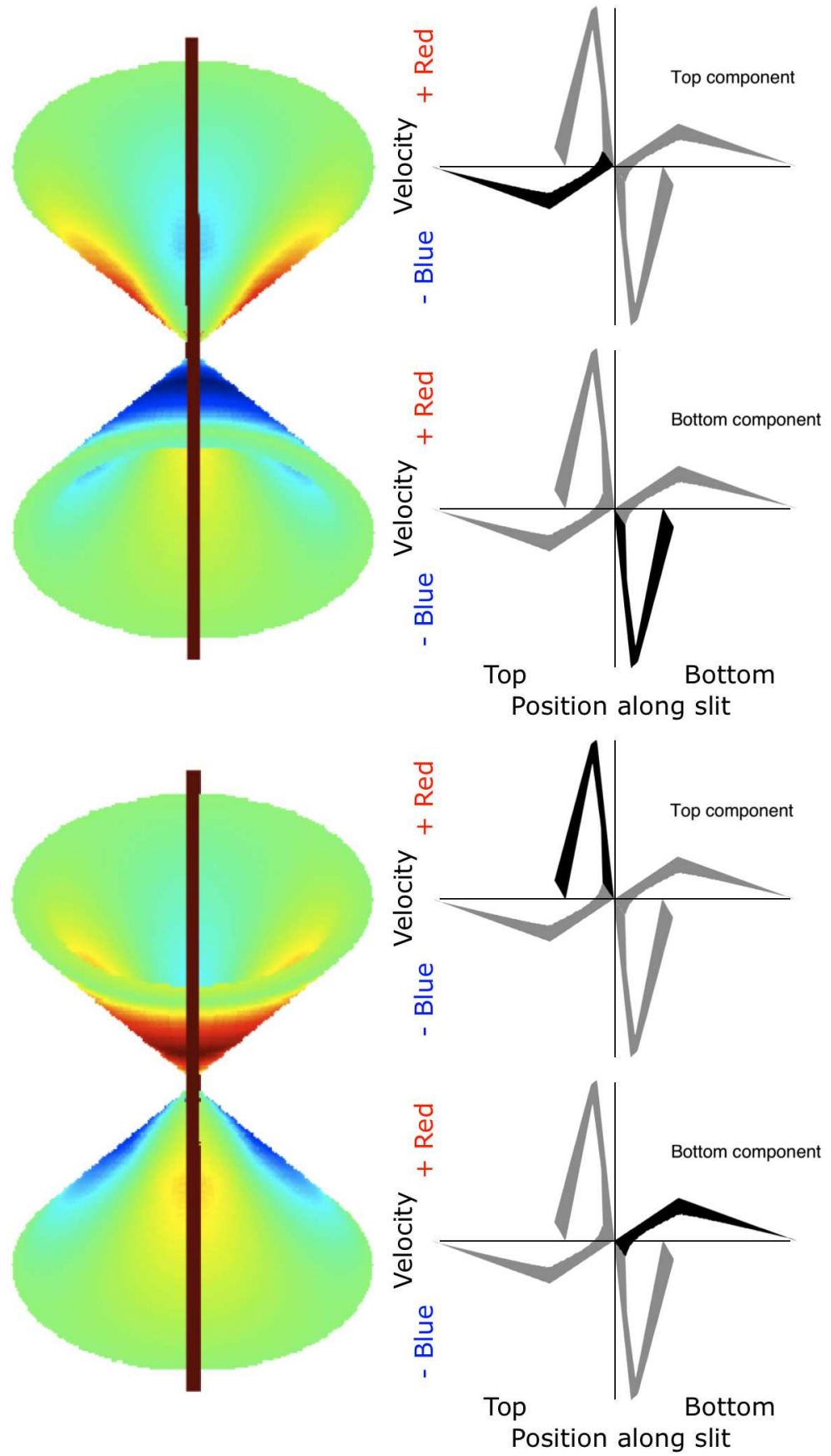

Figure 3.2 Front (top) and back (bottom) sides of a radial velocity map model from our line of sight. Model axis inclination is $30^{\circ}$ out of the plane of the sky, such that the bottom cone is inclined toward Earth. Blue colors represent blueshifted outflow, red colors represent redshifted outflow. The red slit represents the orientation of the STIS long-slit observation and resultant extracted velocity plot showing radial velocity (positive, redshifted velocities increasing upward) as a function of position along the slit. The velocity component for each quadrant is highlighted relative to the total extraction from the slit as a visual aid. 
4) If a fit is not suitable for the given data, the parameters are intuitively adjusted in an iterative process. The most important parameter is the inclination of the bicone axis as it relates to the Seyfert type. Thus, a frequent course of action is to vary the inclination and then make adjustments to the rest of the parameters to offset any resulting changes. Model generation, slit extraction, and plotting are repeated until an acceptable match to the data

is determined. A suitable fit must account for most velocities, have a geometry that agrees with imaging, and adheres to the unified model.

5) Errors for a fit are defined as a range of values over which each individual parameter can vary without significantly altering the fit between model and data. For example, we can typically vary $i$ by $\pm 5^{\circ}$ while maintaining a good fit.

Successfully fitting a kinematic model to the velocity data does not require information from all four components. Only two adjacent components (i.e. two components from the same cone or two components closest to Earth) are required in order to see the effects of changing any of the model parameters. This means that a model can be obtained for targets that suffer from extinction via the host disk which can obscure large portions of the NLR. For example, an entire cone can be obscured by the galactic disk and we could still model the other cone.

\subsection{Incorporating the Host Disk}

A final parameter in determining the fit of a model is the interaction between the NLR and the host disk. Adding the geometry of the host disk to that of the NLR allows us to check the compatibility of the kinematic model with available imaging. Imaging that depicts a single cone of emission, for example, must be compatible with a kinematic model that both fits the spectral data and places one cone of the NLR outflow behind the host disk. Disk inclinations and position angles for each modeled AGN are listed in Table 6.4 and were generally determined by fitting isophotes to the host disk. Kinney et al. (2000) chose 
isophotes corresponding to a surface brightness level of 24-25 $B$ mag $\operatorname{arcsec}^{-2}$, which is often deep enough to avoid bar and oval distortion problems. Assuming a circular host galaxy disk, inclinations were determined from the measured ellipticity and the relation $\cos i=b / a$. Errors for inclination and P.A. range between $1^{\circ}-2^{\circ}$ and $2^{\circ}-6^{\circ}$ respectively. When it was not predetermined from imaging which side of the inclined disk was closer to Earth, orientations that most favorably agreed with the observed NLR geometry were employed. 


\section{CHAPTER 4}

\section{MRK 573: A PILOT STUDY ${ }^{1}$}

We chose to study Mrk 573 as our first target because its morphology follows the same pattern seen in Mrk 3, another Seyfert galaxy that had recently been studied via STIS longslit spectra by our group (Crenshaw et al. 2010a). With knowledge of a similar target to provide guidance moving forward, Mrk 573 appeared to be an excellent candidate to study.

Mrk 573 has been thoroughly studied in different wavebands, as it houses a bright AGN. It is a Seyfert 2 galaxy, though spectropolarimetric observations via Subaru (Nagao et al. 2004) revealed broad $\mathrm{H} \alpha\left(\mathrm{FWHM} \approx 3000 \mathrm{~km} \mathrm{~s}^{-1}\right), \mathrm{H} \beta$, and Fe II multiples, confirming the presence of Mrk 573's hidden BLR.

With a recession velocity of $c z=5160( \pm 10) \mathrm{km} \mathrm{s}^{-1}$ from H I 21-cm emission (Springob et al. 2005), Mrk 573 is at a distance of $\sim 72.6 \mathrm{Mpc}\left(\right.$ for $H_{0}=71 \mathrm{~km} \mathrm{~s}^{-1} \mathrm{Mpc}^{-1}$ ); hence $1^{\prime \prime}$ corresponds to a transverse size of $340 \mathrm{pc}$. The host galaxy contains a triple radio source (Ulvestad \& Wilson 1984) which is comprised of a central component and a pair of lobes which lie along a position angle of $\sim 125^{\circ}$. Extended [O III] and [N II] $\lambda \lambda 6548,6583$ plus $\mathrm{H} \alpha$ emission along the radio axis shows a biconical morphology (Tsvetanov \& Walsh 1992; Pogge \& De Robertis 1995).

Previous analysis by Schlesinger et al. (2009) used STIS long-slit spectra to map the kinematics across the NLR in a study of the outflow and feedback of Mrk 573. This study determined that shock ionized radiation is not significant in the disk. Kraemer et al. (2009) also used sophisticated photoionization models to show that photoionization dominates as the source of emission for Mrk 573's inner NLR, similar to Mrk 3 (Collins et al. 2005, 2009), NGC 1068 (Kraemer \& Crenshaw 2000a,b), and NGC 4151 (Kraemer et al. 2000; Crenshaw \& Kraemer 2005, 2007).

\footnotetext{
${ }^{1}$ This chapter was originally published in the Astronomical Journal Vol. 140 under the title "Modeling the Outflow in the Narrow-Line Region of Markarian 573: Biconical Illumination of a Gaseous Disk" (Fischer et al. 2010)
} 
Strong high-ionization forbidden lines ([Fe VII] $\lambda 6087,[\mathrm{Fe} \mathrm{X}] \lambda 6374,[\mathrm{Ne} \mathrm{V}] \lambda \lambda 3346,3426)$ have been detected in optical spectra of Mrk 573 via ground-based observations (StorchiBergmann et al. 1996; Erkens et al. 1997), indicating that the NLR is more highly ionized than in many Seyfert 2 galaxies (Koski 1978). In fact, high-ionization gas has been found at distances of $\sim 2.5 \mathrm{kpc}$ from the AGN (Storchi-Bergmann et al. 1996). Further information on the physical conditions in the central emission-line knot of the NLR is given in Kraemer et al. (2009).

Ground-based images of Mrk 573 in the V filter (Afanasiev et al. 1996) show a host galaxy disk, with an inclination $i=30^{\circ}$ and a major axis position angle $P . A .=103^{\circ}$ with the closest edge located in the southwest, based on fits to the gas kinematics at distances $>6^{\prime \prime}$ from center. In Figure 4.1, we show a structure map (containing both continuum and line emission) of an F606W image of Mrk 573, generated by processes described by Pogge \& Martini (2002) and Deo et al. (2006). The image shows that the ionized gas regions of the NLR and ENLR have a morphology of arcs and spiral fragments. These formations appear to be connected to the surrounding dust lanes (Quillen et al. 1999; Pogge \& Martini 2002), with the NW section of ionized gas leading to the south spiral arm and the SE section leading to the north arm.

\subsection{Mrk 573: Observations}

We retrieved archival STIS long-slit spectra and Wide Field and Planetary Camera 2 (WFPC2) images of Mrk 573 from the Multimission Archive at the Space Telescope Science Institute (MAST). Spectra were obtained on 2001 October 17 with a $52^{\prime \prime} \times 0.2^{\prime \prime}$ slit using both mediumdispersion G750M and low-dispersion G430L gratings. Further details of each observation are given in Table 4.1; spectral images are shown in Figure 4.2. Observations were dithered by $\pm 0.25^{\prime \prime}$ along the slit with respect to the first spectrum to avoid problems due to hot pixels, and wavelength calibration lamp spectra were taken during Earth occultation. The 


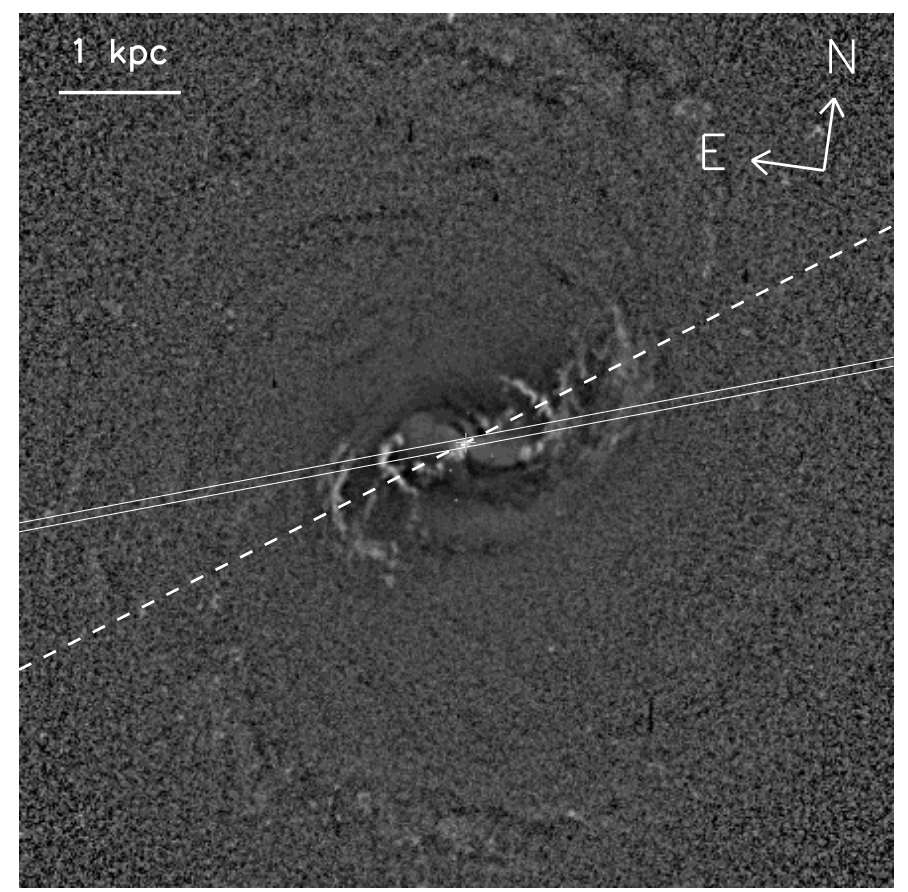

Figure 4.1 Enhanced contrast $20^{\prime \prime} \times 20^{\prime \prime}$ structure map of the HST WFPC2 image of Mrk573, obtained with the F606W filter. Bright areas correspond to line emission and dark areas correspond to dust absorption. Solid lines outline the position of the STIS slit, the dashed line depicts the P.A. of the radio components.

slit for these spectra has a position angle of $-71.2^{\circ}$, favorably overlapping the spatially resolved central knot of [O III] and $\mathrm{H} \alpha$ emission, similar to knots seen in most nearby Seyfert galaxies (Crenshaw \& Kramer 2005), and also crossing several arclike structures in the NLR that have similar strong emission.

Figure 4.1 shows the location of the slit superimposed over a structure map. The location of the optical continuum peak, identified by $\mathrm{a}^{\prime \prime}+{ }^{\prime \prime}$ in the figure, was found by aligning WFPC2 [O III] and continuum images obtained on the same date (Schmitt et al. 2003).

\subsection{Mrk 573: Results}

Velocities were calculated from line centroid shifts as described in Section 2.2, subtracting the systemic velocity of the galaxy to determine the radial velocities along the slit in the 
Table 4.1 HST/STIS Observations of Mrk 573

\begin{tabular}{cccc}
\hline STScI Data Set & Grating & $\begin{array}{c}\text { Central } \lambda \\
(\AA)\end{array}$ & $\begin{array}{c}\text { Exposure } \\
(\mathrm{s})\end{array}$ \\
\hline O6BU02010 & G750M & 6581 & 1080 \\
O6BU02020 & G750M & 6581 & 1080 \\
O6BU02030 & G750M & 6581 & 840 \\
O6BU02040 & G430L & 4300 & 840 \\
O6BU02050 & G430L & 4300 & 805 \\
\hline
\end{tabular}

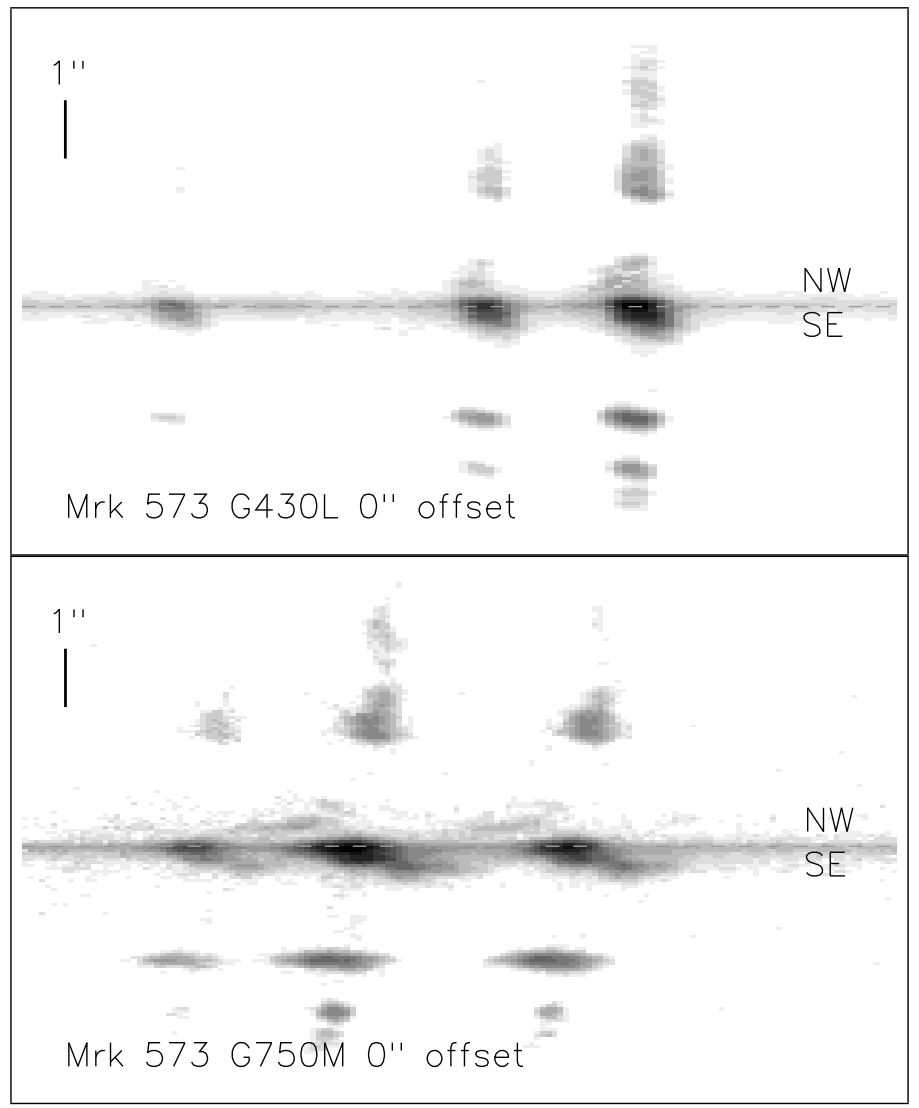

Figure 4.2 HST/STIS long-slit spectral images of Mrk 573. Dashed line gives the location of the continuum centroid. Wavelength increases to the right. Top: H $\beta \lambda 4861$, [O III] $\lambda 4959$, and [O III] $\lambda 5007$ from left to right respectively. Bottom: [N II] $\lambda 6548, \mathrm{H} \alpha \lambda 6563$, and [N II] $\lambda 6583$ from left to right respecitvely. 


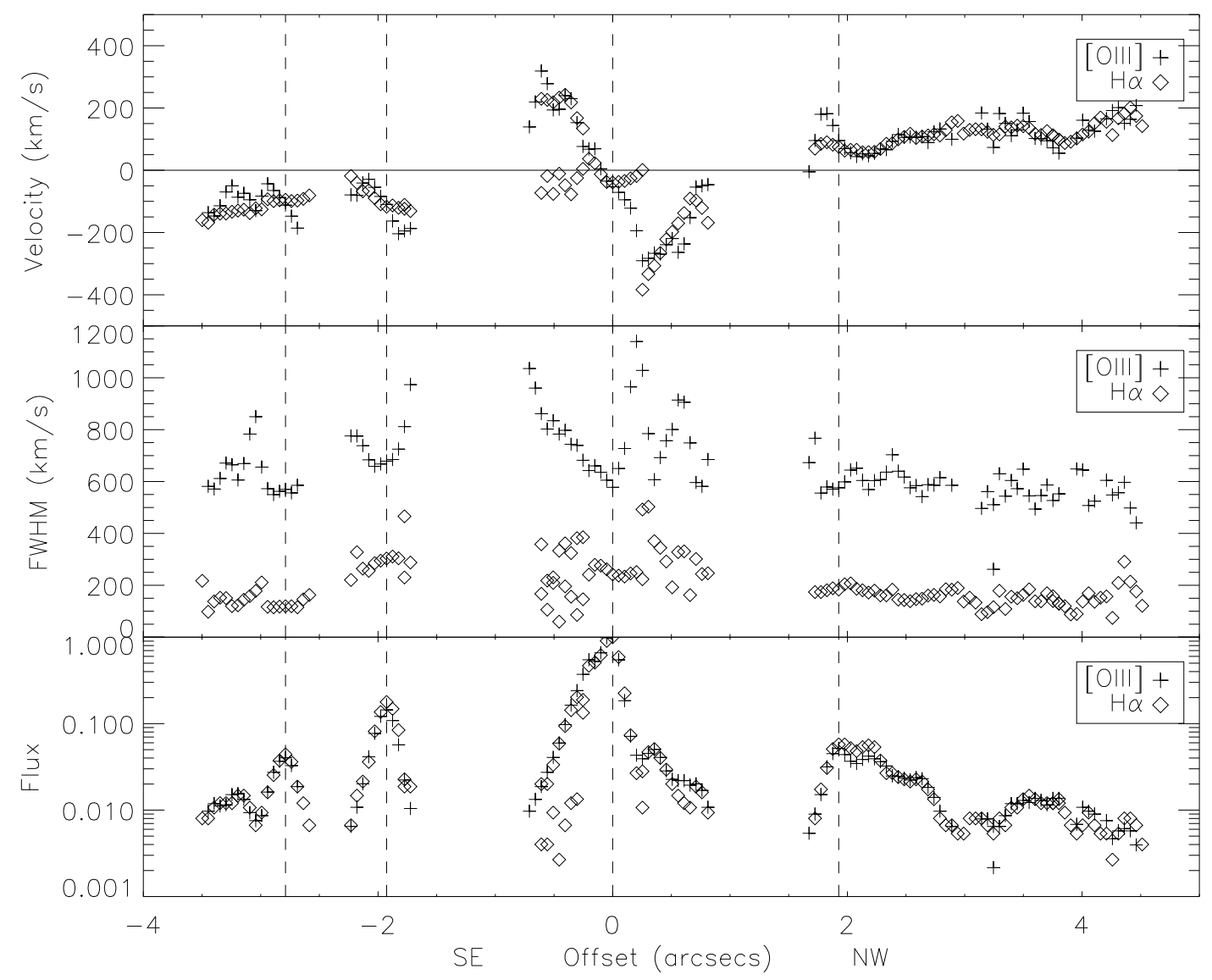

Figure 4.3 Radial velocites (top), FWHM (middle), and normalized fluxes (bottom) of [O III] and $\mathrm{H} \alpha$ measurements for the central slit position of Mrk 573.

rest frame of Mrk 573.

Figure 4.3 shows the rest frame radial velocities, FWHM, and normalized fluxes along the slit for both [O III] $\lambda 5007$ and $\mathrm{H} \alpha \lambda 6563$ emission lines. The four dashed lines represent the central positions of the emission-line arcs from the flux intensity, as well as the central knot at $0^{\prime \prime}$. As mentioned in Section 2.3, discrepancies between the FWHMs of [O III] and $\mathrm{H} \alpha$ are due to different resolutions of the gratings. Comparisons with work by Schlesinger et al. (2009) show that our measurements are in agreement.

Several interesting features are present in both the [O III] $\lambda 5007$ and $\mathrm{H} \alpha$ spectra. On 
either side of the nucleus, high radial velocities show distinct asymetrical red/blue shifts that are characteristic of biconical outflows seen in the previously modeled Seyfert 2 galaxies NGC 1068 (Das et al. 2006) and Mrk 3 (Ruiz et al. 2001; Crenshaw et al. 2010a). Between 0.75" and $1.75^{\prime \prime}$ from the nucleus there is an absence of substantial emission gas; thus there were no measurements in that span. Beyond the empty region, there is emission between $2-4^{\prime \prime}$ on either side of the nucleus that produces linear velocity curves with amplitudes reaching $\sim$ $200 \mathrm{~km} \mathrm{~s}^{-1}$ that Schlesinger et al. (2009) suggested are due to emission in a rotating disk.

\subsection{Mrk 573: Models}

As the STIS slit passes over the NLR emission, we see kinematic components for both sides of the bicone, including a front and back to each half. Our initial model incorporated the narrow bicone observed in the $[\mathrm{O}$ III $]$ image $\left(\theta_{\max }=68^{\circ}\right)$, which proved to be difficult to model as we adopted an acceleration / deceleration model which fit the inner, high radial velocity curve at $<1^{\prime \prime}$ as accurately as possible. In doing so, the outflow in each half of the bicone was either entirely redshifted or blueshifted. This was an unacceptable model as the kinematics showed velocities on either side of the nucleus that are both redshifted and blueshifted. Thus, in order to match our model with the data, we needed to widen the opening angle such that the kinematic component nearest the plane of the sky for each bicone half passed beyond the plane so that its outflow was shifted in the opposite direction of the inner, high velocity kinematic component. To widen the opening angle while maintaining the accurate fit of this inner component required an additional adjustment of the inclination away from our line of sight so that the modeled outflow kinematics near the nucleus would remain successfully fit. Furthermore, while attempting to fit our kinematics model to our data, we needed to account for the circumstances of Mrk 573 uncharacteristic of previous bicone outflow. Whereas the kinematics of our adopted velocity laws contains four peaked radial velocity curves (Das et al. 2005, 2006), only the two inner, high velocity curves in the 


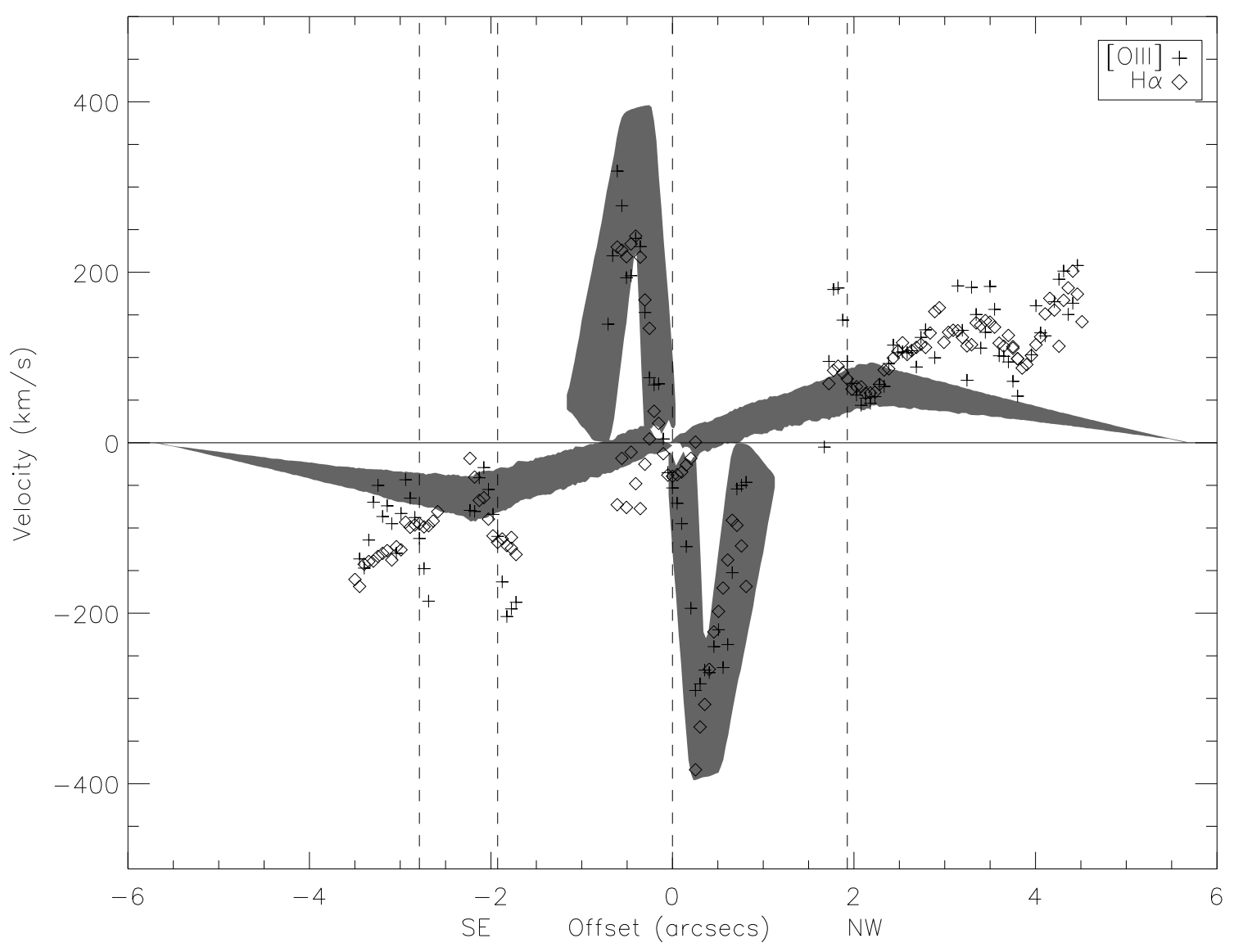

Figure 4.4 Kinematics model chosen as the best fit for our radial velocity data set. Parameters used to create this model are given in Table 4.2. Extended, lower velocity measurements outside $2^{\prime \prime}$ from the nucleus are not enclosed in the model parameters as our model does not account for interactions with a disk.

kinematics of Mrk 573 contain peaks. In the outer, lower velocity curves, we see a continued linear increase in radial velocities which our modeling program was not able to account for. Fits for these outer velocity curves were made such that the increasing slope of the model coincided with the data set, as shown in our model that best fits the data in Figure 4.4. The points not fit by the model are discussed below.

After creating a reasonable kinematic model, the actual outer opening angle of the extended emission at distances $>1^{\prime \prime}$ was found to be much larger than the narrow opening 
angle we observe in Figure 4.1. From the presence of arcs in the ENLR that appear to extend into the outer dust lanes of the galaxy, and similar circumstances occuring in Mrk 3 (Crenshaw et al. 2010a), it is likely that the opening angle seen in images of Mrk 573 is a projection effect due to an intersection of the outflow and the inner galactic disk. This intersection provided us with an additional constraint in which to define the geometry of the emission line regions. We used the best-fit model bicone's $\theta_{\max }$, position angle, and inclination values to create a simple, three-dimensional geometric model of the ionizing bicone's outer surface. We then included a two-dimensional galactic disk, using the observed inclination and postion angles, $30^{\circ}$ and $103^{\circ}$ respectively (Kinney et al. 2000), in the model to show the likely spatial interaction between the two components. This created a projected opening angle upon the model disk that we compared with the emission seen in both the structure map in Figure 4.1. Establishing this second model allowed us to check whether our kinematics model then provided an accurate description of emission seen in imaging of Mrk 573. We altered our parameters in an iterative process until a reasonable kinematic model corresponded with a geometic model whose bicone edges were within $5^{\circ}$ of the imaged emission region, a discrepency that could be due to the unmodeled thickness of the disk.

The model that fits this requirement is shown in Figure 4.5 and a frame of the model is superimposed over the structure map from Figure 4.1 in Figure 4.6 to show how well it fits over the imaged emission, where shaded regions represent portions of the bicone not covered by the disk. Though our model uses a two-dimensional plane for the galactic disk, we can assume a typical dust scale height value of $\sim 200$ pc for spiral galaxies (Xilouris et al. 1999) and a length of $\sim 1500 \mathrm{pc}$ for the bicone along its axis taken from the [O III] image, allowing for much of the bicone edge that lies near the plane of the sky to still interact with the disk. Thus, a reasonable thickness for the inner galactic disk could explain the small discrepancies between our model and the observations in Figure 4.6.

Final values of all model parameters are provided in Table 4.2. Note that our line of sight 


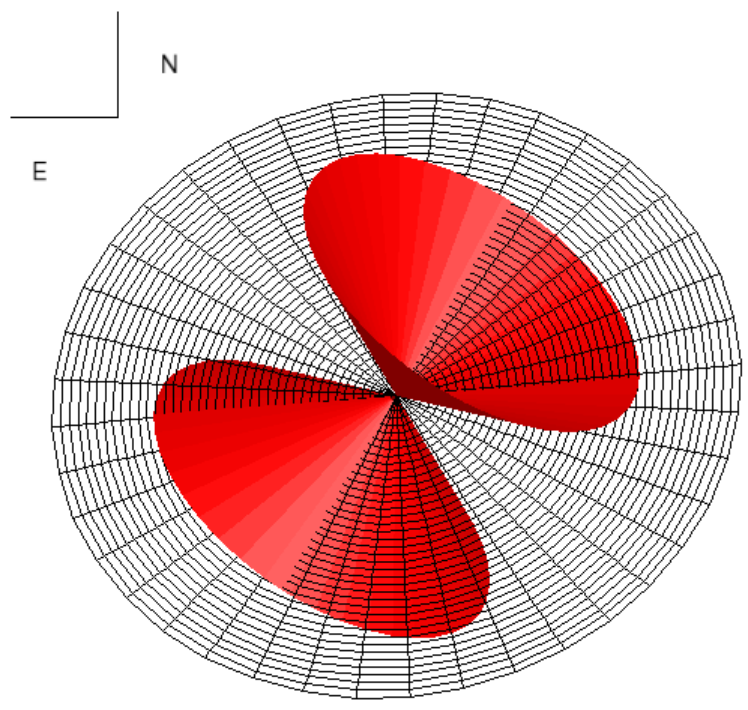

Figure 4.5 Geometric model of the NLR and inner disk in Mrk 573, based on parameters from Table 4.2, shown as viewed from Earth.

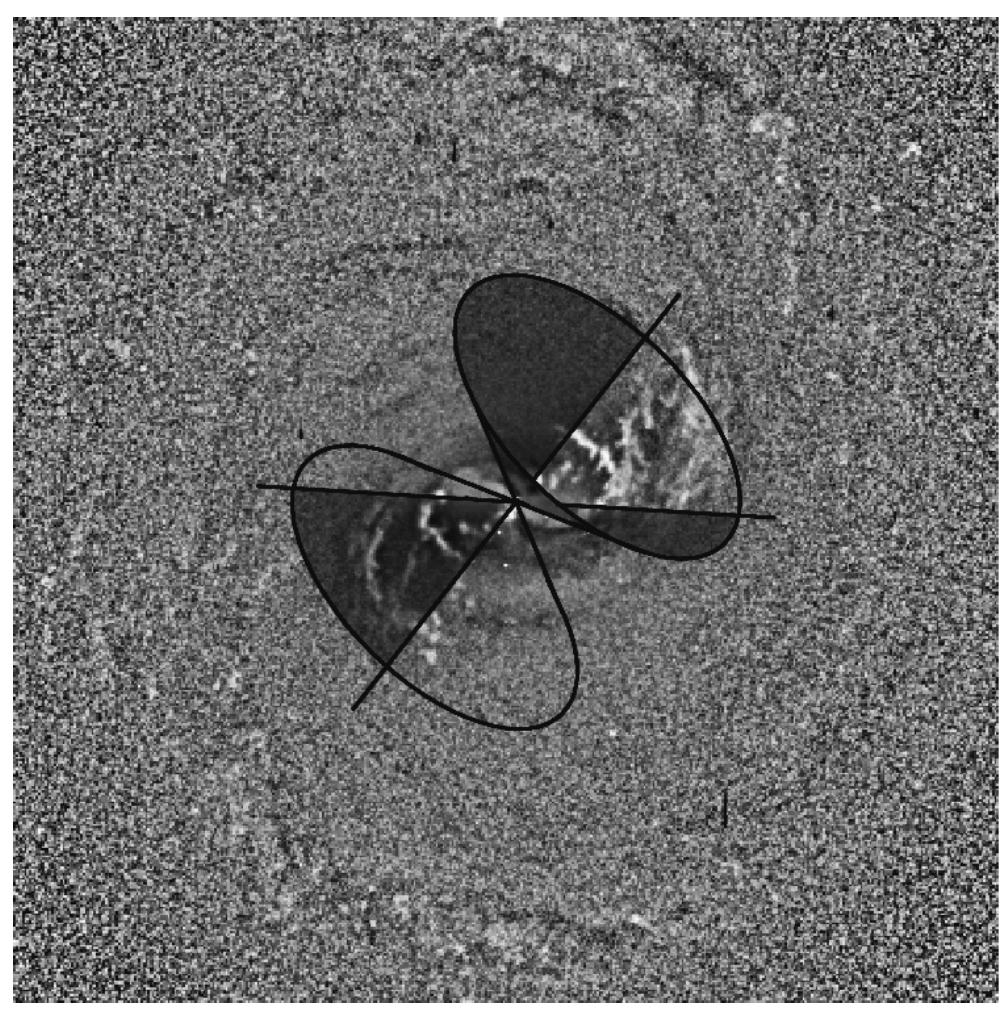

Figure 4.6 Structure map of Mrk 573 with superimposed geometrical model. Shaded regions represent the surface of the bicone in front of the disk intersection along our line of sight. 
Table 4.2 Final Parameters for Mrk 573

\begin{tabular}{cc}
\hline Parameter & Value \\
\hline$P . A$. & $-36^{\circ}$ \\
$i$ & $30^{\circ}(\mathrm{NE})$ \\
$\theta_{\max }$ & $53^{\circ}$ \\
$\theta_{\min }$ & $51^{\circ}$ \\
$v_{\max }$ & $400 \mathrm{~km} \mathrm{~s}^{-1}$ \\
$z_{\max }$ & $1200 \mathrm{pc}$ \\
$r_{t}$ & $800 \mathrm{pc}$ \\
\hline
\end{tabular}

is $8^{\circ}$ outside of the bicone, consistent with the Seyfert 2 nature of Mrk 573. Clearly, the geometric and kinematic constraints of our models lead to significantly different parameters for the ionizing bicone compared to the apparent values from a quick inspection of an image $\left(\mathrm{PA}=-36^{\circ}\right.$ vs. $-52.5^{\circ}, \theta_{\max }=53^{\circ}$ vs. $\left.34^{\circ}\right)$.

\subsection{Mrk 573: Discussion}

The remaining issue in our kinematic model is the lack of deceleration in the outer, low velocity kinematic components, at projected distances $>2^{\prime \prime}(\sim 700 \mathrm{pc})$, as shown in Figure 4.4. Where the bicone kinematics of our previously studied AGN contain four sets of accelerating and decelerating outflows (Das et al. 2005, 2006; Crenshaw et al. 2010a), only Mrk 573's two inner kinematic components follow this pattern. We suggest that the easily fit kinematic components at $\pm 1^{\prime \prime}$ are dominated by emission from the outflow, while extended emission outside $\pm 1.75^{\prime \prime}$ is dominated by disk gas interacting with the bicone of ionizing radiation. As such, it is the presence of the disk that may be responsible for the absence of peaks present in the outflows of other Seyfert NLRs.

One possible reason we see large radial velocities at distances $>2^{\prime \prime}$ is that they are due to emission from a rotating disk (Schlesinger et al. 2009), which our modeling program does not account for. This suggestion immediately seems plausible as images like Figure 4.1 
show emission from arcs within the ENLR that may be joined with dust lanes further out. However, there are a couple of potential problems with this hypothesis. With Mrk 573's host disk inclination being $30^{\circ}$ out of the plane of the sky, a deprojection of the extended velocities would reach speeds of $\sim 400 \mathrm{~km} \mathrm{~s}^{-1}$. Sparke \& Gallagher (2000) note that while velocities this large are not unheard of, with the fastest measured rotation being $\sim 500 \mathrm{~km} \mathrm{~s}^{-1}$ in UGC 12591, they are rare. Using a warped disk (Lawrence \& Elvis 2010) in an attempt to explain the kinematics via rotation could bring down the deprojected velocities. Warping the inner disk further out of the plane of the sky would reduce the difference between the projected and actual velocities creating a more plausible argument for the outer kinematics to be due to rotation. What warping does not fix, however, is the direction in which the disk is rotating. Our kinematics data show the southeast end of the slit to be blueshifted and the northwest end to be redshifted. However, Mrk 573's clockwise spinning morphology in Figure 4.1, along with having the southwest side of the disk being nearest to us, suggests that the northwest region should be blueshifted and southeast redshifted, as nearly all spiral galaxies rotate in the sense that their arms appear to "wind up" (de Vaucouleurs 1958; Toomre 1981). Thus, Mrk 573 may be a candidate for showing leading spiral structure which, similar to NGC 4622 (Buta et al. 2003), may result from a minor merger or tidal encounter. However, once again, this would be a rare circumstance. We also checked the possibility that the disk is inclined in the opposite direction $\left(-30^{\circ}\right.$ instead of $\left.30^{\circ}\right)$. Making this alteration in the geometrical model has the disk almost parallel with the bicone axis, effectively bisecting each half. This is a problem because we do not see a projected opening angle large enough to agree with the model (roughly twice as wide). Furthermore, this model does not agree with our finding from the kinematics data that the bicone is hollow along its axis. Both problems led us to conclude that a reversed inclination is not likely. H I 21-cm observations would likely be the best means to clarify the large scale rotational structure.

If these kinematics are indeed not due to rotation, another option is in situ acceleration 
of gas off the emission-line arcs due to radiative driving or entrainment by highly ionized winds. Work by Das et al. (2007) on NGC 1068 shows radiative acceleration can drive ionized clouds of gas outward at these large distances. Although we do not have any kinematic data between $0.75^{\prime \prime}$ and $1.75^{\prime \prime}$ from the nucleus, there are three velocity gradients (centered on dashed lines located at $\sim-2.75^{\prime \prime},-2^{\prime \prime}$, and $1^{\prime \prime}$ in Figure 4.3) that coincide with the positions of the emission-line arcs along the slit seen in the structure map of Figure 4.1. All three arcs' velocities are redshifted on the southeast side and blueshifted on the northwest side. These gradients are possibly due to the superposition of the slit on a projected outward expansion of the arcs occuring along the intersection between the arcs and the edge of the bicone flowing through the disk. This interaction would create curved annular shells around the edge of the bicone that, from our point of view and using the given position of the slit, could allow us to see the expanding shell's rear redshifted region first and then the front blueshifted region as we go from the southeast end of the long-slit to the northwest. This is strong evidence that the emission-line gas may be accelerating off the arcs, which are likely continuations of the dust spirals and may represent the original fueling flow to the AGN, as we have suggested for Mrk 3 (Crenshaw et al. 2010a).

Comparing our derived opening angle for Mrk 573 against the projected opening seen in HST images, as well as against opening angles given in previous works (Schlesinger et al. 2009; Wilson \& Tsvetanov 1994), our value is much larger and consistent with those of other recently studied Seyfert 2s, Mrk 3 and NGC 4151 (Das et al. 2005; Crenshaw et al. 2010a). These examples may not be unique and large opening angles are likely common for Seyfert galaxies. In targets where most of the observed NLR / ENLR emission comes from the interaction between the ionizing radiation and the galactic disk, similar to Mrk 573 and Mrk 3, observed opening angles can be much smaller than their true opening angles. Data from Mrk 573, Mrk 3, NGC 4151, and NGC 1068 (Das et al. 2006) support the fact that we only see $[\mathrm{O}$ III] and $\mathrm{H} \alpha$ around the edges of the bicone. Thus, if observed targets have a 
biconical outflow that is not apparently hollow at the center (ie a filled triangular projection of emission in the plane of the sky) and show evidence of an interaction with the galactic disk, we can assume an intersection between the galactic disk and an edge of the bicone. Intersections closer to the axis of the bicone would result in projecting an illuminated "V" as the disk interacts with the edges of the outflow bicone and not the inner, hollowed region. This being said, any observed opening angle for targets matching the above description, similar to Mrk 573, would likely be smaller than the true opening angle.

\subsection{Mrk 573: Conclusions}

By generating kinematic and geometric models of the NLR and ENLR of Mrk 573, we were able to fit them successfully to our data and determinine parameters of Mrk 573's outflow bicone. We found that most of the circumnuclear emission comes from the intersection of the galactic disk with the bicone of ionizing radiation. This finding is supported by the presence of emission arcs that appear to be related to the outer dust lanes as well as the success of our geometrical model. Similarities between Mrk 573 and Mrk 3 suggest that the emission could be due to ionization of the original fueling flow (Crenshaw et al. 2010a), which is thought to occur along the dusty spirals (Pogge \& Martini 2002).

Kinematics outside projected distances of 700 pc from the nucleus could possibly be due to rotation, although this seems unlikely due to a large deprojected amplitude, large velocity dispersion, and requirement of leading spiral structure. Alternatively, we might be seeing in situ acceleration of gas off the previously non-emitting inner dust/gas spiral arms. The latter more easily explains the large velocity gradients at each emission arc.

The half-opening angle we find from our models $\left(53^{\circ}\right)$ is similar to previously studied Seyfert 2s Mrk 3 and NGC 4151 and is larger than the ratio of Seyfert 1s to Seyfert 2s would predict. Osterbrock \& Shaw (1988) used statistics of numbers of Seyfert 1, 1.5, 1.8, 1.9, and 2 galaxies in Wasilewski field to find that $78 \%$ of field AGN were type 2, leading 
them to calculate an average torus half-opening angle of approximately $35^{\circ}$. With Mrk 573 , we now have three very clear type 2 Seyferts objects with opening angles bigger than this

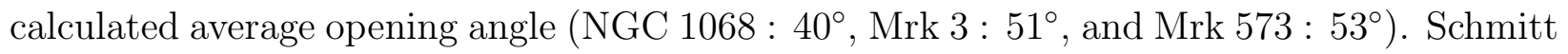
et al. (2001) calculated a half opening angle of $48^{\circ}$, a value similar to our results, by binning Seyfert 1.8s and 1.9s with Seyfert 2s to determine the ratio of Seyfert 1s to 2s. Continued research on opening angles of individual Seyfert galaxies is required to determine if these large opening angles are unique. Should we find values consistently above those determined from the Seyfert 1 / Seyfert 2 ratio, we must question if simple obscuration by tori explains the relative fraction of Seyfert 1s to Seyfert 2s. For the opening angle to be so large and depend solely on the torus for its definition, the estimated percentage of Seyfert 2s observed would be less than what we actually see. It is possible that some secondary property could be accountable for the fraction of Seyfert $2 \mathrm{~s}$ that we see, such as the presence of obscuring gas which is separate from the torus, although possibly connected to the creation of dusty circumnuclear gas that makes a torus. Additional modeling of the interaction between the NLR outflow and the galactic disk supports our suggestion that any observed opening angle for Seyfert galaxies exhibiting an interaction between the outflow and the galactic disk could be much smaller than the true value due to projection effects. If continued research on determining geometric parameters in Seyfert galaxies finds opening angles greater than both observed and statistically predicted, obscuration from non-toroidal components might be accountable for the large percentage of Seyfert 2 galaxies that we see. 
CHAPTER 5

\section{MRK 78: AN APPLICATION OF OUTFLOW MODELING ${ }^{1}$}

Observations of two distinct sets of emission lines at different radial velocities in a spectrum have been used to indicate the presence of dual AGN due to galaxy mergers, which are thought to be important for supermassive black hole growth (Hopkins et al. 2007). There has been considerable discussion around the study of double emission lines as to whether they are clearly an indication for the presence of multiple AGN in a single galaxy (Komossa et al. 2008a,b; Comerford et al. 2009), or they can be attributed to radial velocity offsets due to mass outflows in a single AGN (Crenshaw et al. 2010b). With nearby examples present for both explanations, it is of great interest to understand the kinematics of these targets as much as possible in hopes of applying this knowledge to higher redshift double-emission line analogs.

A particular AGN of this variety is the Seyfert 2 galaxy Mrk 78. With a redshift of $z$ $=0.036793$ based on Ca T stellar absorption lines (Nelson \& Whittle 1995), Mrk 78 is at a distance of $\sim 150 \mathrm{Mpc}$ (for $H_{0}=73 \mathrm{~km} \mathrm{~s}^{-1} \mathrm{Mpc}^{-1}$ ); at this distance, $1^{\prime \prime}$ corresponds to a transverse size of $\sim 730$ pc. Mrk 78 has been extensively studied due to a visual correspondence between its radio source and extended narrow-line region (ENLR) (Whittle et al. 2002; Whittle \& Wilson 2004; Whittle et al. 2005). HST FOC images of Mrk 78 in [O III] show that its narrow-line region (NLR) consists of two highly luminous regions extending over $\sim 5^{\prime \prime}(\sim 3.6 \mathrm{kpc})$ that is in a biconical geometry also seen in several other Seyfert 2 galaxies (Schmitt et al. 2003). We chose Mrk 78 for this study as ground-based spectroscopic observations of Mrk 78 have shown that its narrow emission lines are double peaked (Sargent 1972; Adams 1973). Ground-based spectra at high spectral resolution depict the [O III]

\footnotetext{
${ }^{1}$ This chapter was originally published in the Astrophysical Journal Vol. 727 under the title "HST Observations of the Double-Peaked Emission Lines in the Seyfert Galaxy Markarian 78: Mass Outflows from a Single AGN" (Fischer et al. 2011)
} 
emission-line profile as two large, well-defined peaks separated by $\sim 800 \mathrm{~km} \mathrm{~s}^{-1}$ (Whittle et al. 1988; Nelson \& Whittle 1995).

Crenshaw et al. (2010b) showed that velocity offsets between the narrow emission lines and host galaxy lines can be explained by the combination of mass outflows and dust extinction in AGN, or even an asymmetric distribution of outflowing gas. They also suggested that these explanations could possibly work for double-lined AGN, such as Mrk 78, as well. Previous studies had already suggested that radial motion via inflows or outflows could be responsible for observed narrow line asymmetries and velocity shifts (Whittle 1985; Dahari \& De Robertis 1988; De Robertis \& Shaw 1990), including long-slit ground-based observations that gave evidence for the production of asymmetries via outflows and dust obscuration (Storchi-Bergmann et al. 1992; Arribas et al. 1996; Christopoulou et al. 1997; Ramos Almeida et al. 2006; Rodríguez-Ardila et al. 2006). In Fischer et al. (2011), we used high resolution spectroscopy and imaging to show that the velocity offset seen in Mrk 78 can indeed be explained by mass outflows in the NLR, as discussed below.

\subsection{Mrk 78: Observations}

We obtained archival STIS long-slit spectra and Faint Object Camera (FOC) images of Mrk 78 from the Multimission Archive at the Space Telescope Science Institute (MAST). Four sets of spectra were taken between 1998 February 28 and 1998 March 01 with a $52^{\prime \prime} \times 0.2^{\prime \prime}$ slit using a G430M grating. Of the four observations, three were taken at a position angle of $88^{\circ}$ and the fourth was taken at a position angle of $61^{\circ}$. The calibrated spectral images for each slit position are shown in Figure 5.1. Additional details of each observation are given in Tables 5.1 and 5.2 .

Figure 5.2 shows an extended continuum STIS acquisition image of Mrk 78 with additional, contoured [O III] and UV FOC images and positions for the four STIS slits illustrated on the [O III] image. The slits are offset from the optical continuum peak, signified as a 

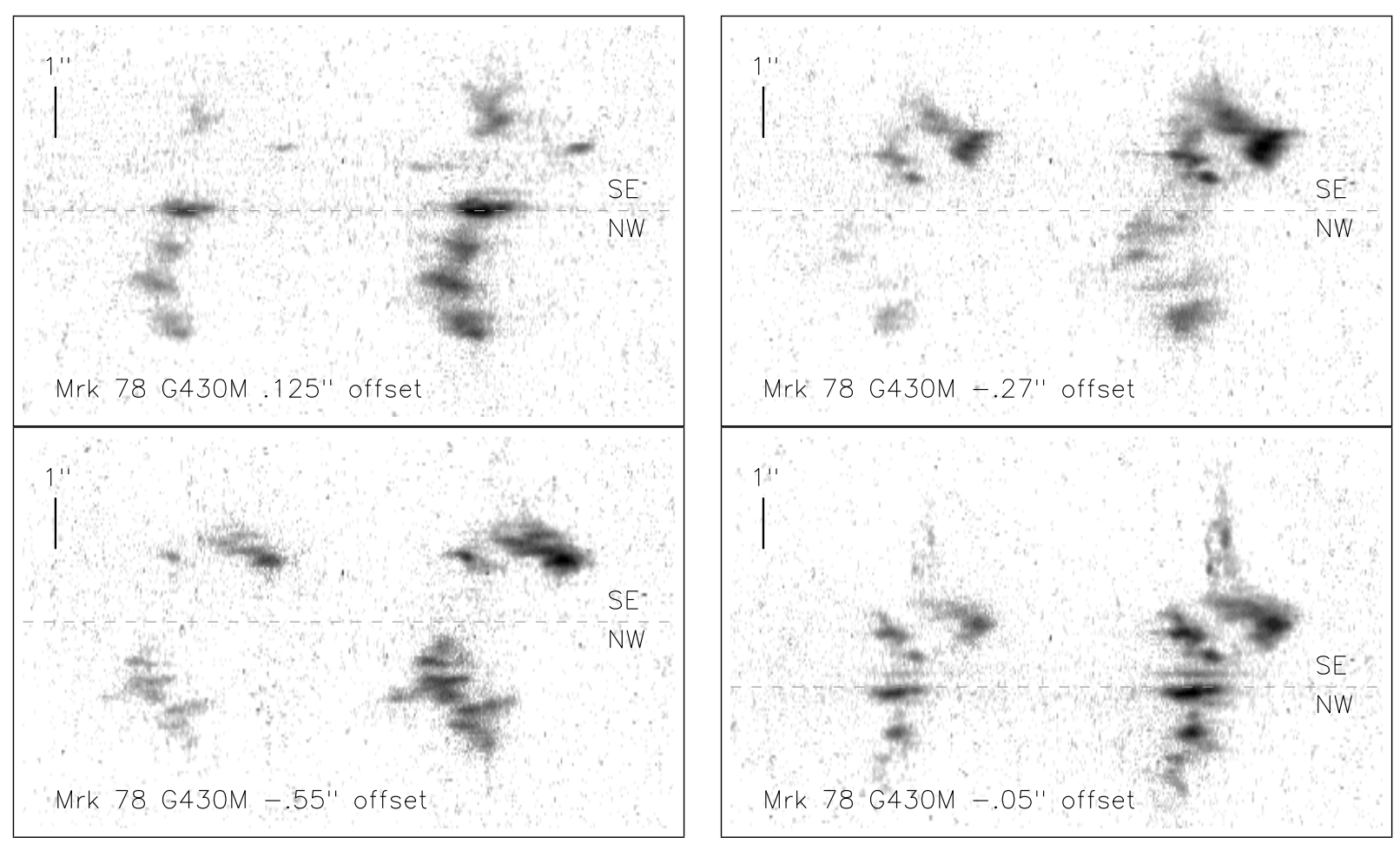

Figure 5.1 HST/STIS long-slit spectral images of Mrk 78. Dashed line gives the location of the continuum centroid. Wavelength increases to the right with [O III] $\lambda 4959$ and [O III] $\lambda 5007$ to the left and right respectively. Slits A - D progress from left to right and top to bottom.

Table 5.1 HST STIS Observations of Mrk 78

\begin{tabular}{cccc}
\hline Slit & $\begin{array}{c}\text { Exposure } \\
(\mathrm{s})\end{array}$ & $\begin{array}{c}\text { P.A. } \\
\left({ }^{\circ}\right)\end{array}$ & $\begin{array}{c}\text { Offset } \\
\left({ }^{\prime \prime}\right)\end{array}$ \\
\hline $\mathrm{A}$ & 1727 & 88 & 0.125 \\
$\mathrm{~B}$ & 1938 & 88 & -0.27 \\
$\mathrm{C}$ & 2052 & 88 & -0.55 \\
$\mathrm{D}$ & 1800 & 61 & -0.05 \\
\hline
\end{tabular}




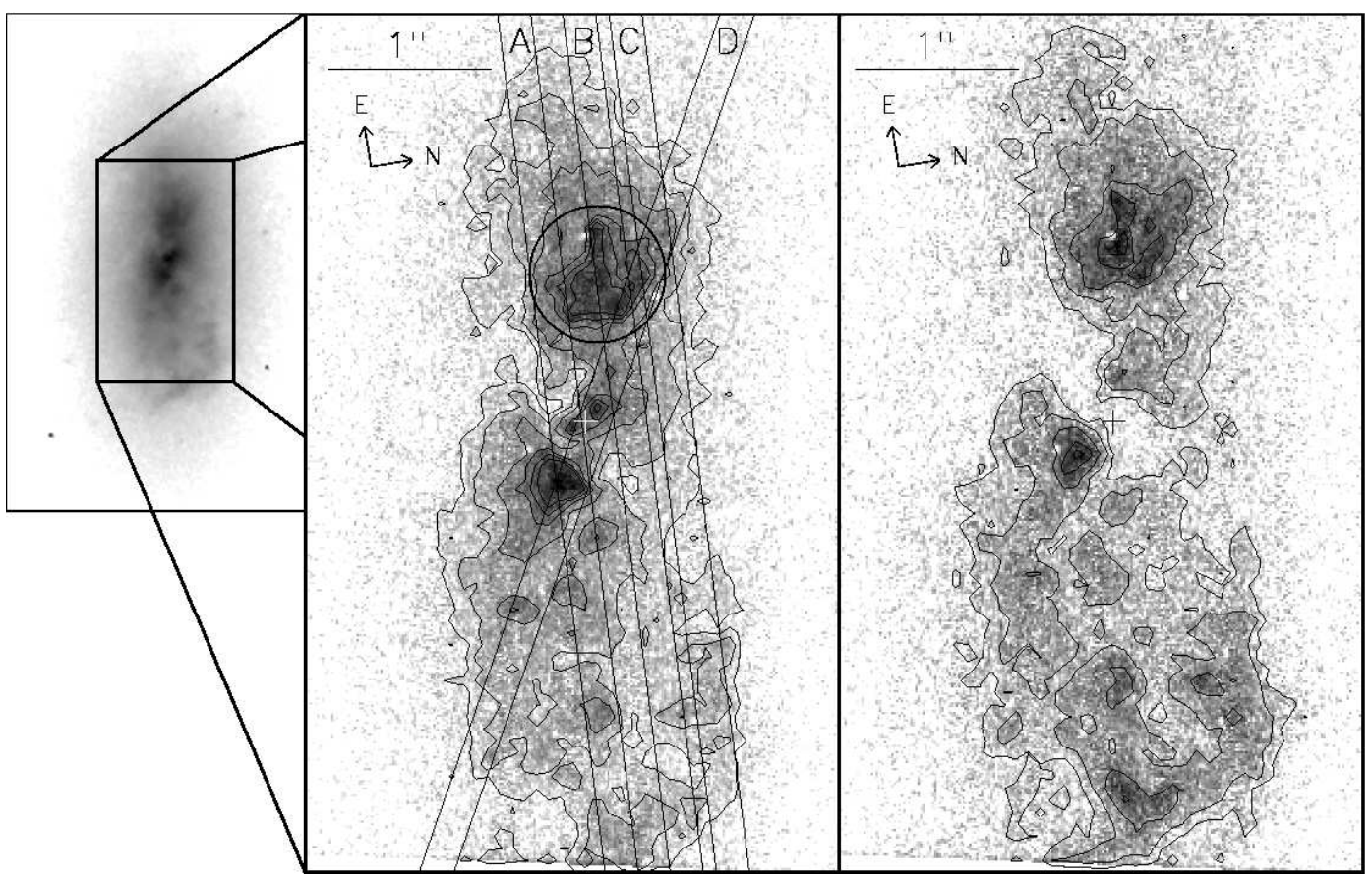

Figure 5.2 Composite of HST STIS acquisition continuum image (left) with additional enlarged [O III] (F502M filter, as seen in Schmitt \& Kinney (1996)) and UV (F372M filter, mainly [OII] emission) FOC images (middle and right respectively). The dust lane sweeping over the AGN is located at a position angle of $\sim 123^{\circ}$ southeast to northwest. STIS slit positions have been superimposed and the red-shifted emission knot responsible for the secondary radial velocity peak is circled on the [O III] image.

Table 5.2 HST Imaging Observations of Mrk 78

\begin{tabular}{ccc}
\hline Instrument & Filter & $\begin{array}{c}\text { Exposure } \\
(\mathrm{s})\end{array}$ \\
\hline STIS & MIRVIS & 120. \\
FOC & $372 \mathrm{M}$ & 896. \\
FOC & $550 \mathrm{M}$ & 1196. \\
FOC & $502 \mathrm{M}$ & 801. \\
\hline
\end{tabular}


" $+"$, which was located by aligning the $[\mathrm{O}$ III $]$ and continuum FOC images taken on the same date (Schmitt et al. 2003) and is also coincident with the infrared peak as observed by Ramos Almeida et al. (2006).

We used the STIS spectra to compare our results with those taken from previous groundbased spectra, obtained through apertures that included most, if not all, of the NLR in Mrk 78 at moderate spectral resolution (80-230 $\mathrm{km} \mathrm{s}^{-1}$ FWHM) (Vrtilek \& Carleton 1985; Nelson \& Whittle 1995). The STIS datasets consist of hundreds of spectra along each slit, allowing us to resolve individual emission-line knots that are responsible for a majority of the emission seen in NLRs. To simulate the ground-based spectra, we combined all of the STIS spectra along each slit position and then combined the spectra from each slit, excluding slit D as it overlaps the other three slits, similar to the procedure in Crenshaw et al. (2010b).

Figure 5.3 shows the final co-added spectrum in terms of flux as a function of the radial velocity of the [O III] line, with respect to the systemic velocity calculated from our redshift. The significantly separated [O III] $\lambda 5007$ peaks agree with those from the moderateresolution ground-based spectra of Nelson \& Whittle (1995), with the higher peak being at near systemic velocity $\left(v_{r}=-50 \mathrm{~km} \mathrm{~s}^{-1}\right)$ and the secondary red peak at $v_{r}=800 \mathrm{~km}$ $\mathrm{s}^{-1}$, meaning any [O III] emission not covered by the STIS long-slit observations does not significantly alter the profile shape or structure.

\subsection{Mrk 78: Results}

Figure 5.4 show the rest frame radial velocities, FWHM, and normalized fluxes for the measured [O III] $\lambda 5007$ emission lines from the four slit positions. Kinematics from the lower spectral resolution IR spectrum of Ramos Almeida et al. (2006) agree with our measurements. In general, the structure of the emitting region is clumpy in nature, as seen in the large number of flux peaks in each slit that can be matched to individual bright knots in the [O III] image of Figure 5.2. While each knot appears to have an individual peculiar velocity, 


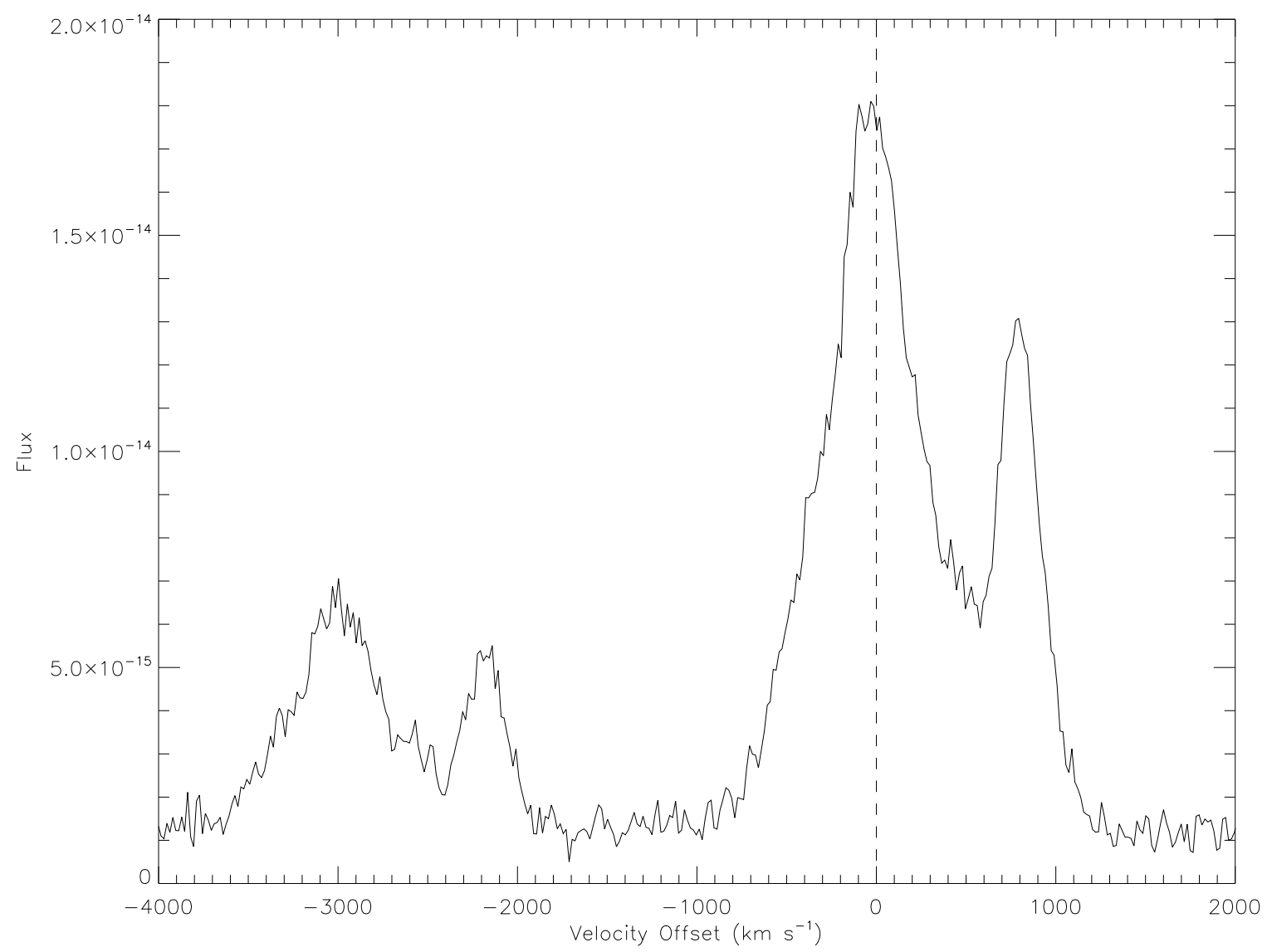

Figure 5.3 Summation of spectral flux from slits A, B, and C as a function of radial velocity for the [O III] $\lambda 5007$ emission line with respect to the systemic velocity. Double peaked [O III] $\lambda 4959$ emission is seen to the left.

the velocity field as a whole is relatively well organized. High radial velocities on each side of the nucleus show the distinct asymmetrical red/blue shifts characteristic of biconical outflows seen in our previous low-redshift Seyfert galaxy studies (Ruiz et al. 2001; Das et al. 2005, 2006; Crenshaw et al. 2010a; Fischer et al. 2010). Emission eastward of the optical peak is predominantly redshifted from systemic velocity (henceforth 'red lobe') while emission westward of the optical peak is predominantly blueshifted (henceforth 'blue lobe'). Breaking down our simulated ground based spectrum of Figure 5.3 into the individual slits A, B, and $\mathrm{C}$, we find that the redshifted velocity peak is primarily due to a specific high-flux knot, best 

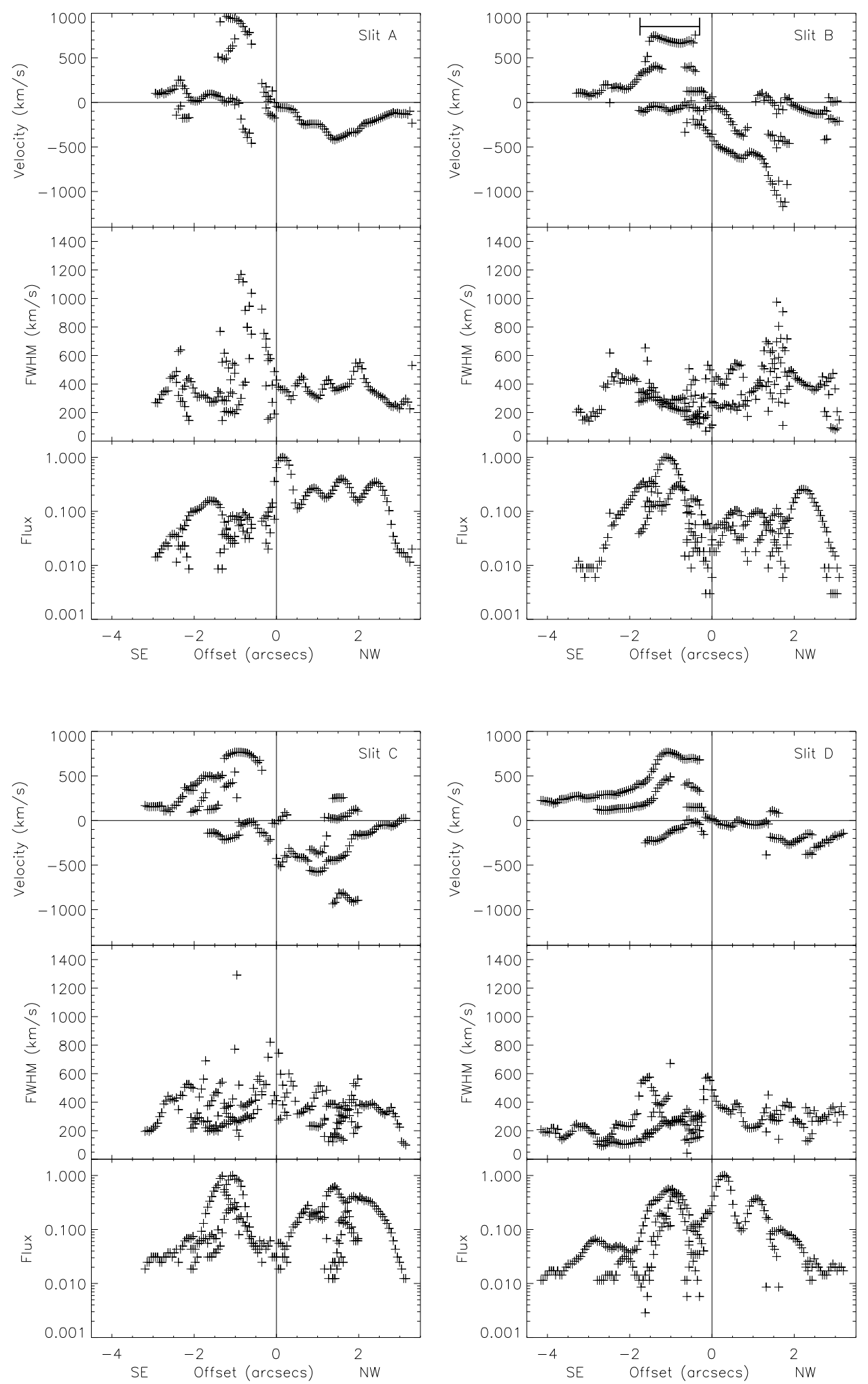

Figure 5.4 Radial velocities (top), FWHM (middle), and normalized total flux (bottom) of [O III] measurements. The bracket in slit B shows the location of the high flux cloud responsible for the secondary radial velocity peak. 
sampled in slit B. Figure 5.2 contains a circle around the location of the redshifted knot and Figure 5.4 has a bracket in slit B, located over the high-flux region which depicts the knot.

\subsection{Mrk 78: Models}

We again employed our kinematics model to recreate the observed radial velocities along a fixed slit position by altering various parameters of our model bicone outflow. ${ }^{2}$

The FOC [O III] image of Figure 5.2 shows that the NLR is not symmetric on either side of the nucleus. The redshifted, NE cone is less dispersed, more conical in nature, and features cleaner kinematics from which we can discern separate components due to outflow emission in the front and back side of the cone. Conversely, the blueshifted, SW cone appears to have a larger opening angle, with flocculent, knotty emission and resulting intricate kinematics. Though using initial parameters solely from the NE cone was enticing, we found that the bicone did not envelop the entirety of the west lobe. Thus, we expanded the model bicone outer opening angle to envelop all NLR emission in the [O III] image in Figure 5.3. We chose $\theta_{\max }=30^{\circ}$ as our initial opening angle and used the kinematic components of the NE cone as the basis for the majority of our fitting. The initial inclination parameter relied on the kinematics seen in Figure 5.4, as either side was either mostly redshifted or blueshifted, and the imaging in Figure 5.2 as the extended biconical structure on either side of the nucleus would be less pronounced if we were to be looking into the bicone (Schmitt \& Kinney 1996). With these guidelines, we determined an intermediate angle was most appropriate for a starting position. To find the most successful model, we altered parameters across all data sets while applying specific centroid offsets and position angles for each slit until a specific

\footnotetext{
${ }^{2}$ Previous studies of Mrk 78 have suggested that the NLR outflow is being radially accelerated via radio jets (Whittle \& Wilson 2004; Whittle et al. 2005). While our model does not include any specific source of acceleration, instead simply simulating general outward acceleration, we do not detect considerable differences in the velocities or dispersions of emission-line knots near radio knots from others in the overall outflow as noted in Whittle et al. (2002).
} 
Table 5.3 Final Parameters for Mrk 78

\begin{tabular}{cc}
\hline Parameter & Value \\
\hline$P . A$. & $65^{\circ}$ \\
$i$ & $30^{\circ}(\mathrm{SW})$ \\
$\theta_{\max }$ & $35^{\circ}$ \\
$\theta_{\min }$ & $10^{\circ}$ \\
$v_{\max }$ & $1200 \mathrm{~km} \mathrm{~s}^{-1}$ \\
$z_{\max }$ & $3200 \mathrm{pc}$ \\
$r_{t}$ & $700 \mathrm{pc}$ \\
\hline
\end{tabular}

set of free parameters created an acceptable fit to all four data sets. Final values for all model parameters are provided in Table 5.3.

Interestingly, the final opening angle and position angle are nearly identical to the apparent, initial parameters taken from Figure $5.2\left(\theta_{\max }=30^{\circ}\right.$ and $P . A .=70^{\circ}$ respectively). In comparison to our previous studies (Ruiz et al. 2001; Das et al. 2005, 2006; Fischer et al. 2010), Mrk 78 has an extremely large NLR as well as a narrower, thicker (greater difference between $\theta_{\max }$ and $\theta_{\min }$ ) bicone than all other targets thus far.

Figure 5.5 displays a good qualitative fit between the model created by our final set of parameters and the data. The model matches the observations fairly well to the east of the nucleus as kinematics for both sides of the eastern lobe are discernible in multiple slits. One discrepancy worth discussing is the lack of model data (shaded regions) mainly to the west of the nucleus in Slits B and C between $-0.25^{\prime \prime}$ to $0.5^{\prime \prime}$ and $-0.5^{\prime \prime}$ to $1.5^{\prime \prime}$, respectively. As our kinematic model creates a well defined geometrical model bicone with a sharp apex, incorporating slit positions that are transversely offset from the nucleus will generate models that lack data near the nucleus, as the slit does not pass over the model bicone. This is due to the simplicity of our assumption of a sharp apex, which is obviously not the case in this and many other Seyferts (Antonucci 1993; Crenshaw et al. 2010b; Fischer et al. 2010). Thus, Slits B and C lack modeled kinematics in these regions as the actual emission lies outside 

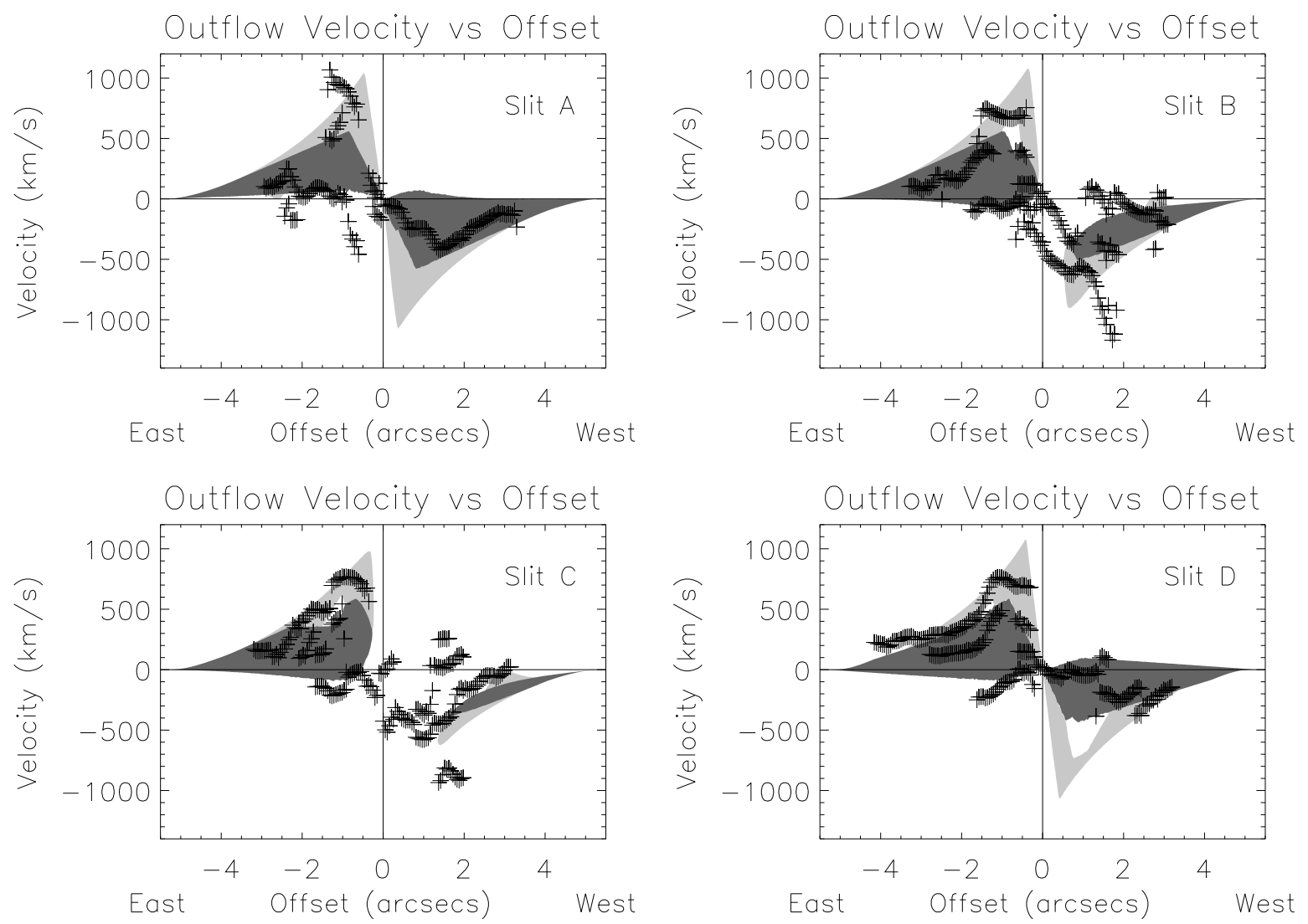

Figure 5.5 Shaded regions represent the kinematic model chosen as the best fit for our radial velocity data set for all four slits. Parameters used to create this model are given in Table 5.3. Lighter regions represent kinematics for the side of each cone near parallel to our line of sight, darker regions represent kinematics for the side near perpendicular to our line of sight.

our model bicone geometry. Additionally, unfit high-velocity data points between $1^{\prime \prime}$ and $2^{\prime \prime}$ west of the nucleus in Slits B and $\mathrm{C}$ were not given precedence when determining our final model as they are the tertiary fit, low flux components that are less likely related to the NLR outflow.

\subsection{Mrk 78: Discussion}

The available evidence indicates that Mrk 78 is not a double AGN. Observing the variance in flux across the slit, we see many bumps and peaks that are due to large, bright knots of 
emission-line gas, each with their own peculiar velocity inside the general outflow pattern. It is one of these knots, a bright red-shifted cloud traveling at $\sim 800 \mathrm{~km} \mathrm{~s}^{-1}$ inside of the ionizing bicone, that is the source of the offset radial velocity peak. The cloud appears to fit well in our modeled outflow pattern, supporting the suggestion by Crenshaw et al. (2010b) that uneven outflow can account for such dual-peaked profiles, and thus there is no need to assume there is a second AGN present. In comparison to our previous studies, kinematics for the NLR in Mrk 78 actually look rather similar to those in NGC 4151 (Hutchings et al. 1998; Crenshaw et al. 2000; Das et al. 2005), NGC 1068 (Crenshaw \& Kraemer 2000; Das et al. 2006, 2007), Mrk 3 (Ruiz et al. 2001; Crenshaw et al. 2010a), and Mrk 573 (Schlesinger et al. 2009; Fischer et al. 2010). For each of these targets, including Mrk 78, we have successfully employed our biconical outflow models to find a good match to the overall flow patterns as a function of distance from the central AGN. Though there are some inconsistencies between the model and the data that are likely due to the simplified geometry of our bicone model and peculiar velocities of individual emission-line knots, radial outflow of [O III] clouds can explain the general, large-scale flow pattern of the NLR in Mrk 78, as well as the double peaked emission.

Does obscuration from dust contribute to the creation of the dual-peaked radial velocity profile as mentioned in Crenshaw et al. (2010b)? Ground based images of Mrk 78 in B and I give a position angle and inclination of the host galaxy as $84^{\circ}$ and $64^{\circ}$ respectively $($ Schmitt \& Kinney 2000; Kinney et al. 2000). Adding this geometry to a geometric bicone model, we can test to see if a smooth distribution of dust contributed by the disk could be responsible for the radial velocity profile. Figure 5.6 shows simple, three-dimensional geometric models of the ionizing bicone's outer surface using $\theta_{\max }$, position angle, and inclination values from our final kinematics model and an additional two-dimensional galactic disk using the above position angle and inclination values. Though it is unknown which side of the disk is closer, either geometry in combination with our final bicone model results in a radial velocity profile 


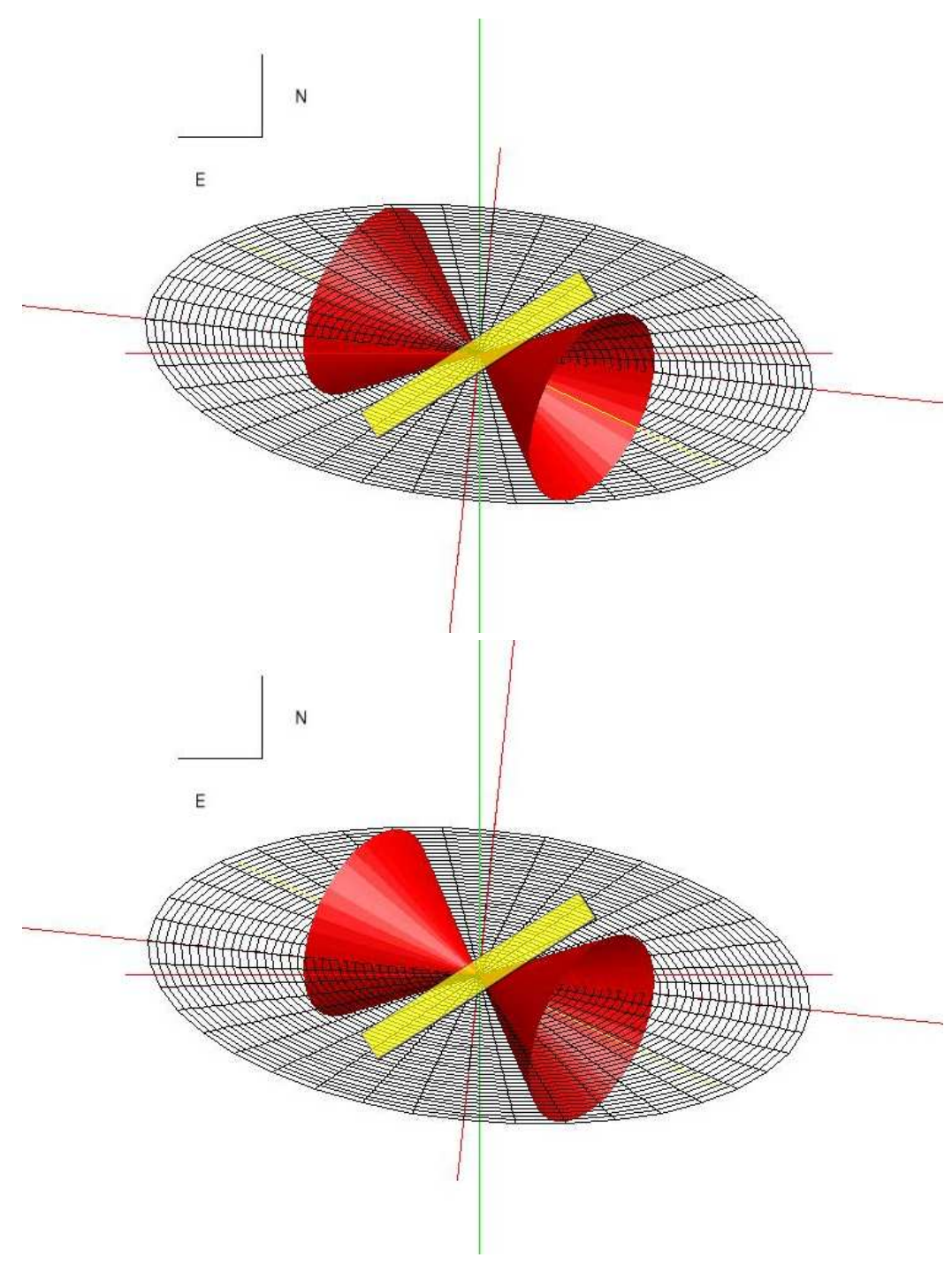

Figure 5.6 Geometric models of the NLR based on parameters from Table 5.3, shown as viewed from Earth. Inner disk geometry is taken from Kinney et al. (2000) depicting: (top) the top of the disk inclined closer to Earth; (bottom) the bottom of the disk inclined closer to Earth. The yellow bar represents the position angle of the dust lane traveling over the nucleus. The bright redshifted knot is in the northeast portion of the bicone.

that is either symmetric or asymmetric towards the blue. The latter case can be seen in the bottom of Figure 5.6 as the redshifted cone should be more extinguished by dust in the galactic plane. This scenario does not fit our data as the blue-shifted, likely less obscured lobe would then contribute a majority of the total emission-line flux. Thus, it is unlikely 
that a dusty disk would be responsible for the given profile.

There is a prominent dust lane that sweeps over the center of the AGN. Each image in Figure 5.2 shows a distinct band from southeast to northwest across the nucleus with a position angle of $\sim 123^{\circ}$ that has flux much lower than the emission surrounding it. More quantitatively, we obtained an additional STIS G750M spectrum from MAST taken along the same position as the G430M slit D in order to measure the flux ratio of $H \alpha / H \beta$ over the nucleus, and calculated a reddening of $E(B-V)=0.78$ (assuming a galactic reddening curve and intrinsic $H \alpha / H \beta=2.9)$. The reddening is significant enough to extinguish portions of the inner NLR, possibly suppressing high-velocity dispersion emission from the bicone at $\sim 0 \mathrm{~km} \mathrm{~s}^{-1}$ and making the double line profile more distinct. However, this reddening is not enough to extinguish the AGN broad-line region (BLR). As such, it is likely that this dust lane must be additional obscuration external to the "torus".

Further evidence for a dust lane can be seen in the blue-shifted southwest lobe from the imaging shown in Figure 5.2. From the end of the absorbing dust lane, an arc of emission is visible along the north rim of the lobe $\sim 2^{\prime \prime}$ from the " $+{ }^{\prime \prime}$ marking the continuum peak location. It remains unclear whether the dust lane crossing across the nucleus arcs over the southwest lobe and continues to absorb emission or instead arcs into the ionizing bicone and becomes illuminated, similar to Mrk 3 and Mrk 573 (Crenshaw et al. 2010a; Fischer et al. 2010). However, both situations would help explain the asymmetrical, patchy emission seen in the blue-shifted emission lobe. The top model in Figure 5.6 depicts a galactic disk with the north side of the disk coming out of the page, which allows for a potential interaction between the disk and ionizing bicone. A full understanding of this extended morphology will require more thorough imaging before a more accurate claim can be made. 


\subsection{Mrk 78: Conclusions}

While it is not clear at this time how the blue-shifted lobe is interacting with the dust lane lying over the nucleus, it is likely that that interaction can explain the dispersed morphology present in the given FOC and STIS imaging. We find that the double peaked emission lines seen in ground-based spectra of Mrk 78 are due to an asymmetric distribution of outflowing gas in the NLR. Specifically, the red peak at $+800 \mathrm{~km} \mathrm{~s}^{-1}$ is due to a bright emission-line knot that is redshifted with respect to the systemic velocity of the host galaxy. The bright, redshifted knot follows the same flow pattern as the other knots, and thus, there is no need to assume that a second AGN resides within Mrk 78. With nothing unusual separating Mrk 78 from other AGN, it is possible that many double-peaked profiles may be due to outflows in a single AGN as shown by our models. 


\section{CHAPTER 6}

\section{EXPANDING THE SEYFERT AGN SAMPLE}

In order to determine if any observed properties of AGN are correlated with their inclinations, we required a larger sample than the 5 individual AGN inclinations currently published by our group. Thus, we expanded our sample to include all Seyfert AGN observed with STIS using the G430L and G430M gratings, which include the bright [O III] $\lambda 5007$ emission line.

\subsection{Observations}

In total, the expanded sample, not including the five previously modeled AGN, contains 161 spectra of 47 AGN. Observation parameters, including slit orientation, offset distance from the continuum flux peak, and the source of the observation, are listed in Table 6.1. Spectral images of all long-slit observations are depicted in Appendix B.

\subsubsection{MAST Archive Spectra}

This expansion harvested the remaining non-proprietary archival G430L/M long-slit spectra from MAST for 31 additional AGN. Additional G750M observations that exist for targets selected in this matter were also harvested, in an effort to test the consistencies seen between [O III] and $\mathrm{H} \alpha$ kinematics in Mrk 573. In many cases, multiple observations at the same

position were dithered along the slit with respect to the first spectrum to avoid problems due to hot pixels. Wavelength calibration lamp spectra were taken during Earth occultation.

\subsubsection{Proprietary Spectra}

In addition to the available archive spectra, we obtained new long-slit G430M observations for 11 Seyferts through two HST proposals:

Are Narrow-Line Seyfert 1 Galaxies Viewed Pole-on?: The objective of this proposal (Program ID: 11611, PI: Crenshaw) was to determine inclinations of Narrow Line Seyfert 1 
(NLS1) galaxies. NLS1s have permitted lines with widths from their BLRs that are $\leq 2000$ $\mathrm{km} \mathrm{s}^{-1}$ (FWHM), which are narrower than those of "normal" broad-line Seyfert 1s (BLS1s), but still broader than forbidden lines from the NLR (typically $\sim 500 \mathrm{~km} \mathrm{~s}^{-1} \mathrm{FWHM}$ ). The current paradigm for NLS1s is that they have supermassive black holes (SMBHs) with relatively low masses compared to BLS1s and they are therefore radiating at close to their Eddington limits (Pounds et al. 1995), i.e. L/L $\mathrm{L}_{e d d} \approx 1$. Should NLS1s be more "pole-on" than normal broad-line Seyfert 1s (BLS1s), we could determine if these properties are due instead to a special viewing angle for NLS1s as suggested by their compact radio morphologies (Ulvestad et al. 1995). For example, if the BLR dynamics are dominated by rotation, then a face-on view would lower the calculated SMBH mass and increase $\mathrm{L} / \mathrm{L}_{e d d}$.

Two spectra were obtained per AGN, at roughly perpendicular slit position angles using a reduced slit width of $0.1^{\prime \prime}$. The thinner slit width was employed in an effort to isolate spatially distinct regions in particularly compact NLRs. Ultimately, these observations were unsuccessful; we were able to detect blueshifted emission near each AGN nucleus but we were unable to resolve individual kinematic components from the data.

What are the Locations and Kinematics of Mass Outflows in AGN?: This proposal (Program ID: 12212, PI: Crenshaw) aimed to better understand mass outflows of ionized gas in AGN, seen as blueshifted UV and X-ray absorption lines, as they are likely play an important role in enrichment of the IGM, self-regulation of black-hole growth, and formation of structure in the early Universe through feedback. STIS [O III] observations were included in this proposal as the inclination of each AGN would aid in investigating the connection between nuclear absorption and NLR emission outflows and their dependence on polar angle. Observations for NGC 3516 were not used, as the chosen $0.1^{\prime \prime}$ slit width did not allow for any significant emission to be detected. 


\subsubsection{Slitless Spectra}

To expand our sample even further, we returned to results from Ruiz et al. (2005) to analyze slitless spectra of 10 Seyfert AGN, with 5 targets new to our sample. To quickly summarize their slitless spectral measurement process, emission-line knots were identified in both the direct and grating-dispersed images. Both spatial and spectral images were then fit row by row with a Gaussian for each knot and the radial velocity of each knot was determined by subtracting the positions of the Gaussian peaks in the direct and dispersed images. This created a two-dimensional velocity map where each knot in the image was assigned a radial velocity, which we could pull out in a strip across the image to simulate a long-slit observation. Due to the nature of the slitless observations, only very bright, distinct knots of emission yielded radial velocities. Thus, due to a lack of lines fit from the spectra within $0.2^{\prime \prime}$ slit widths as were measured using long-slit observations, simulated observations were taken in $2^{\prime \prime}$ wide slits. These psuedo-observations, in turn, were then fit with our modeling program in an identical fashion as typical long-slit observations. 
Table 6.1: Expanded Sample: HST/STIS Observations.

\begin{tabular}{|c|c|c|c|c|c|c|c|c|c|}
\hline Target & STScI Data Set & Source & $\begin{array}{l}\text { Date } \\
(\mathrm{UT})\end{array}$ & Grating & Aperture & $\begin{array}{c}\text { Central } \lambda \\
(\AA)\end{array}$ & $\begin{array}{l}\text { P.A. } \\
\text { (deg) }\end{array}$ & $\begin{array}{c}\text { Offset } \\
\text { (arcsec) }\end{array}$ & $\begin{array}{c}\text { Exp. } \\
(\mathrm{s})\end{array}$ \\
\hline \multirow[t]{3}{*}{ Akn 564} & OBGU08010 & $\mathrm{C}$ & $2011 \mathrm{Jul} 31$ & G430M & $52 \times .2$ & 5093 & 159.65 & 0. & 695 \\
\hline & OBGU08010 & $\mathrm{C}$ & 2011 Jul 31 & G430M & $52 \times .2$ & 5093 & 159.65 & 0. & 695 \\
\hline & OBGU08010 & $\mathrm{C}$ & 2011 Jul 31 & G430M & $52 \times .2$ & 5093 & 159.65 & 0. & 695 \\
\hline \multirow[t]{6}{*}{ Circinus } & O65B01040 & $\mathrm{A}$ & 2000 Oct 12 & G430M & $52 \times .2$ & 4961 & 113.65 & -.6 & 1400 \\
\hline & O65B01050 & $\mathrm{A}$ & 2000 Oct 12 & G430M & $52 \times .2$ & 4961 & 113.65 & -.4 & 1443 \\
\hline & O65B01060 & $\mathrm{A}$ & 2000 Oct 12 & G430M & $52 \times .2$ & 4961 & 113.65 & -.2 & 1400 \\
\hline & O65B01070 & A & 2000 Oct 12 & G430M & $52 \times .2$ & 4961 & 113.65 & 0 . & 1495 \\
\hline & O65B01080 & A & 2000 Oct 12 & G430M & $52 \times .2$ & 4961 & 113.65 & +.2 & 1400 \\
\hline & O65B01090 & $\mathrm{A}$ & 2000 Oct 12 & G430M & $52 \times .2$ & 4961 & 113.65 & +.4 & 1492 \\
\hline \multirow[t]{5}{*}{ IC 3639} & O6BU01010 & $\mathrm{A}$ & 2002 Jan 23 & G750M & $52 \times .2$ & 6581 & -104.41 & 0. & 1080 \\
\hline & O6BU01020 & A & 2002 Jan 23 & G750M & $52 \times .2$ & 6581 & -104.41 & 0 . & 1158 \\
\hline & O6BU01030 & $\mathrm{A}$ & 2002 Jan 23 & G750M & $52 \times .2$ & 6581 & -104.41 & 0. & 900 \\
\hline & O6BU01040 & $\mathrm{A}$ & 2002 Jan 23 & G430L & $52 \times .2$ & 4300 & -104.41 & 0. & 840 \\
\hline & O6BU01050 & A & 2002 Jan 23 & G430L & $52 \times .2$ & 4300 & -104.41 & 0. & 823 \\
\hline IRAS 11058 & O56C03050 & $\mathrm{A}$ & 1999 Apr 08 & G430L & $52 \times .2$ & 4300 & 36.03 & 0. & 600 \\
\hline \multirow{6}{*}{ MCG-6-30-15 } & O5GU08010 & A & 2000 Mar 07 & G430M & $52 \times .2$ & 4961 & -75.02 & 0. & 1707 \\
\hline & O5GU08020 & A & 2000 Mar 07 & G430M & $52 \times .2$ & 4961 & -75.02 & 0. & 2511 \\
\hline & O5GU08030 & $\mathrm{A}$ & 2000 Mar 07 & G430M & $52 \times .2$ & 4961 & -75.02 & +.2 & 1250 \\
\hline & O5GU08040 & $\mathrm{A}$ & 2000 Mar 07 & G430M & $52 \times .2$ & 4961 & -75.02 & +.2 & 1192 \\
\hline & O5GU08050 & $\mathrm{A}$ & 2000 Mar 07 & G430M & $52 \times .2$ & 4961 & -75.02 & -.2 & 1250 \\
\hline & O5GU08060 & A & 2000 Mar 07 & G430M & $52 \times .2$ & 4961 & -75.02 & -.2 & 1189 \\
\hline \multirow[t]{4}{*}{ Mrk 34} & O5G404010 & A & 2000 Feb 17 & G430M & $52 \times .2$ & 5216 & 152.48 & 0. & 1500 \\
\hline & O5G404010 & $\mathrm{A}$ & 2000 Feb 17 & G430L & $52 \times .2$ & 4300 & 152.48 & 0. & 627 \\
\hline & O5G404010 & A & 2000 Feb 17 & G430M & $52 \times .2$ & 5216 & 152.48 & +.28 & 1460 \\
\hline & O5G404010 & $\mathrm{A}$ & 2000 Feb 17 & G430M & $52 \times .2$ & 5216 & 152.48 & -.28 & 1460 \\
\hline \multirow[t]{3}{*}{ Mrk 279} & OBGU05010 & $\mathrm{C}$ & 2011 May 10 & G430M & $52 \times .2$ & 5093 & 124.65 & 0. & 712 \\
\hline & OBGU05020 & $\mathrm{C}$ & 2011 May 10 & G430M & $52 \times .2$ & 5093 & 124.65 & 0. & 712 \\
\hline & OBGU05030 & $\mathrm{C}$ & 2011 May 10 & G430M & $52 \times .2$ & 5093 & 124.65 & 0. & 712 \\
\hline \multirow[t]{2}{*}{ Mrk 348} & O5G405010 & A & 1999 Sep 28 & G430M & $52 \times .2$ & 5093 & 145.98 & 0 . & 1410 \\
\hline & O5G405020 & $\mathrm{A}$ & 1999 Sep 28 & G430L & $52 \times .2$ & 4300 & 145.98 & 0. & 600 \\
\hline \multirow{2}{*}{ Mrk 463e } & O5G406010 & A & 2000 Mar 14 & G430M & $52 \times .2$ & 5216 & -178.03 & 0. & 1200 \\
\hline & O5G406020 & A & 2000 Mar 14 & G430L & $52 \times .2$ & 4300 & -178.03 & 0. & 556 \\
\hline
\end{tabular}

Continued on next page 
Table 6.1 Continued from previous page

\begin{tabular}{|c|c|c|c|c|c|c|c|c|c|}
\hline Target & STScI Data Set & Source & $\begin{array}{l}\text { Date } \\
\text { (UT) }\end{array}$ & Grating & Aperture & $\begin{array}{c}\text { Central } \lambda \\
(\AA)\end{array}$ & $\begin{array}{l}\text { P.A. } \\
\text { (deg) }\end{array}$ & $\begin{array}{c}\text { Offset } \\
\text { (arcsec) }\end{array}$ & $\begin{array}{c}\text { Exposure } \\
(\mathrm{s})\end{array}$ \\
\hline \multirow[t]{3}{*}{ Mrk 493} & O92X16010 & $\mathrm{A}$ & 2004 Jul 28 & G430L & $52 \times .2$ & 4300 & 74.65 & 0. & 720 \\
\hline & O92X16020 & A & 2004 Jul 28 & G430L & $52 \times .2$ & 4300 & 74.65 & 0 . & 720 \\
\hline & O92X16030 & A & 2004 Jul 28 & G430L & $52 \times .2$ & 4300 & 74.65 & 0 . & 720 \\
\hline \multirow[t]{3}{*}{ Mrk 509} & OBGU07010 & $\mathrm{C}$ & 2011 Aug 07 & G430M & $52 \times .2$ & 5093 & 74.65 & 0 . & 695 \\
\hline & OBGU07020 & $\mathrm{C}$ & 2011 Aug 07 & G430M & $52 \times .2$ & 5093 & 74.65 & 0 . & 695 \\
\hline & OBGU07030 & C & 2011 Aug 07 & G430M & $52 \times .2$ & 5093 & 74.65 & 0. & 695 \\
\hline \multirow[t]{2}{*}{ Mrk 705} & OB1105010 & B & 2010 Dec 10 & G430M & $52 \times .1$ & 5093 & -100.35 & 0 . & 2148 \\
\hline & OB1106010 & B & 2011 Feb 10 & G430M & $52 \times .1$ & 5093 & 7.92 & 0 . & 2148 \\
\hline \multirow[t]{2}{*}{ Mrk 766} & OB1101010 & B & 2010 Mar 21 & G430M & $52 \times .1$ & 5093 & 129.65 & 0. & 2148 \\
\hline & OB1102010 & B & 2010 Dec 23 & G430M & $52 \times .1$ & 5093 & -120.35 & 0 . & 2148 \\
\hline \multirow[t]{2}{*}{ Mrk 1040} & OB1103010 & B & 2009 Jul 02 & G430M & $52 \times .1$ & 5093 & -144.20 & 0 . & 2148 \\
\hline & OB1104010 & B & 2009 Nov 14 & G430M & $52 \times .1$ & 5093 & 119.65 & 0 . & 2148 \\
\hline \multirow[t]{3}{*}{ Mrk 1044} & OBGU01010 & $\mathrm{C}$ & 2011 Nov 06 & G430M & $52 \times .2$ & 5093 & -36.23 & 0. & 695 \\
\hline & OBGU01020 & C & 2011 Nov 06 & G430M & $52 \times .2$ & 5093 & -36.23 & 0 & 695 \\
\hline & OBGU01030 & $\mathrm{C}$ & 2011 Nov 06 & G430M & $52 \times .2$ & 5093 & -36.23 & 0. & 695 \\
\hline \multirow{2}{*}{ Mrk 1066} & O5G407010 & A & 2000 Oct 30 & G430M & $52 \times .2$ & 4961 & 130.65 & 0 . & 1440 \\
\hline & O5G407020 & A & 2000 Oct 30 & G430L & $52 \times .2$ & 4300 & 130.65 & 0 . & 600 \\
\hline \multirow[t]{5}{*}{ NGC 1358} & O6BU03010 & A & $2002 \operatorname{Jan} 25$ & G750M & $52 \times .2$ & 6581 & 23.89 & 0. & 1080 \\
\hline & O6BU03020 & A & 2002 Jan 25 & G750M & $52 \times .2$ & 6581 & 23.89 & 0 . & 1080 \\
\hline & O6BU03030 & A & 2002 Jan 25 & G750M & $52 \times .2$ & 6581 & 23.89 & 0. & 840 \\
\hline & O6BU03040 & A & 2002 Jan 25 & G430L & $52 \times .2$ & 4300 & 23.89 & 0 & 840 \\
\hline & O6BU03050 & A & 2002 Jan 25 & G430L & $52 \times .2$ & 4300 & 23.89 & 0 . & 805 \\
\hline NGC 1386 & O5F402030 & $\mathrm{D}$ & 2000 Jun 23 & G430M & 50CCD & 4961 & 175.82 & 0 . & 2106 \\
\hline \multirow[t]{5}{*}{ NGC 1667} & O6BU04010 & A & 2001 Oct 14 & G750M & $52 \times .2$ & 6581 & -120.21 & 0 . & 1080 \\
\hline & O6BU04020 & A & 2001 Oct 14 & G750M & $52 \times .2$ & 6581 & -120.21 & 0 . & 1080 \\
\hline & O6BU04030 & A & 2001 Oct 14 & G750M & $52 \times .2$ & 6581 & -120.21 & 0 . & 840 \\
\hline & O6BU04040 & A & 2001 Oct 14 & G430L & $52 \times .2$ & 4300 & -120.21 & 0 . & 840 \\
\hline & O6BU04050 & A & 2001 Oct 14 & G430L & $52 \times .2$ & 4300 & -120.21 & 0 . & 805 \\
\hline \multirow[t]{5}{*}{ NGC 2110} & O5G401010 & A & 2000 Dec 24 & G430M & $52 \times .2$ & 4961 & -36.20 & 0 . & 1522 \\
\hline & O5G401020 & A & 2000 Dec 24 & G430M & $52 \times .2$ & 4961 & -36.20 & +.68 & 600 \\
\hline & O64F02010 & A & 2000 Dec 30 & G750M & $52 \times .2$ & 6581 & -24.35 & 0 . & 1440 \\
\hline & O64F02020 & A & 2000 Dec 30 & G750M & $52 \times .2$ & 6581 & -24.35 & 0 . & 1440 \\
\hline & O64F02030 & A & 2000 Dec 30 & G750M & $52 \times .2$ & 6581 & -24.35 & 0 . & 1440 \\
\hline \multirow{2}{*}{ NGC 2273} & O6BU05010 & A & 2001 Nov 04 & G750M & $52 \times .2$ & 6581 & -151.61 & 0. & 1140 \\
\hline & O6BU05020 & A & 2001 Nov 04 & G750M & $52 \times .2$ & 6581 & -151.61 & 0. & 1226 \\
\hline
\end{tabular}


Table 6.1 Continued from previous page

\begin{tabular}{|c|c|c|c|c|c|c|c|c|c|}
\hline Target & STScI Data Set & Source & $\begin{array}{l}\text { Date } \\
\text { (UT) }\end{array}$ & Grating & Aperture & $\begin{array}{c}\text { Central } \lambda \\
(\AA)\end{array}$ & $\begin{array}{l}\text { P.A. } \\
\text { (deg) }\end{array}$ & $\begin{array}{c}\text { Offset } \\
\text { (arcsec) }\end{array}$ & $\begin{array}{l}\text { Exposure } \\
\text { (s) }\end{array}$ \\
\hline & O6BU05030 & $\mathrm{A}$ & 2001 Nov 04 & G750M & $52 \times .2$ & 6581 & -151.61 & 0 & 900 \\
\hline & O6BU05040 & $\mathrm{A}$ & 2001 Nov 04 & G430L & $52 \times .2$ & 4300 & -151.61 & 0. & 840 \\
\hline & O6BU05050 & $\mathrm{A}$ & 2001 Nov 04 & G430L & $52 \times .2$ & 4300 & -151.61 & 0. & 951 \\
\hline \multirow{5}{*}{ NGC 3081} & O6BU06010 & $\mathrm{A}$ & 2001 Dec 04 & G750M & $52 \times .2$ & 6581 & -110.52 & 0. & 1080 \\
\hline & O6BU06020 & $\mathrm{A}$ & 2001 Dec 04 & G750M & $52 \times .2$ & 6581 & -110.52 & 0. & 1080 \\
\hline & O6BU06030 & $\mathrm{A}$ & 2001 Dec 04 & G750M & $52 \times .2$ & 6581 & -110.52 & 0. & 840 \\
\hline & O6BU06040 & $\mathrm{A}$ & 2001 Dec 04 & G430L & $52 \times .2$ & 4300 & -110.52 & 0 . & 840 \\
\hline & O6BU06050 & $\mathrm{A}$ & 2001 Dec 04 & G430L & $52 \times .2$ & 4300 & -110.52 & 0. & 812 \\
\hline \multirow{8}{*}{ NGC 3227} & O57204010 & $\mathrm{A}$ & 1999 Jan 31 & G750M & $52 \times .2$ & 6581 & -137.62 & -.75 & 2105 \\
\hline & O57204020 & $\mathrm{A}$ & 1999 Jan 31 & G750M & $52 \times .2$ & 6581 & -137.62 & -.5 & 1600 \\
\hline & O57204030 & A & 1999 Jan 31 & G750M & $52 \times .2$ & 6581 & -137.62 & -.25 & 1884 \\
\hline & O57204040 & $\mathrm{A}$ & 1999 Jan 31 & G750M & $52 \times .2$ & 6581 & -137.62 & 0 & 1890 \\
\hline & O57204050 & $\mathrm{A}$ & 1999 Jan 31 & G750M & $52 \times .2$ & 6581 & -137.62 & +.25 & 1600 \\
\hline & O57204060 & $\mathrm{A}$ & 1999 Jan 31 & G750M & $52 \times .2$ & 6581 & -137.62 & +.5 & 1884 \\
\hline & O57204070 & $\mathrm{A}$ & 1999 Jan 31 & G750M & $52 \times .2$ & 6581 & -137.62 & +.75 & 1887 \\
\hline & O5KP01020 & $\mathrm{A}$ & 2000 Feb 08 & G430L & $52 \times .2$ & 4300 & -150.34 & 0. & 120 \\
\hline \multirow[t]{5}{*}{ NGC 3393} & $\mathrm{O} 56 \mathrm{C} 02010$ & $\mathrm{~A}$ & 1999 Apr 22 & G750M & $52 \times .2$ & 6581 & 39.98 & -.3 & 1080 \\
\hline & $\mathrm{O} 56 \mathrm{C} 02030$ & $\mathrm{~A}$ & 1999 Apr 22 & G750M & $52 \times .2$ & 6581 & 39.98 & 0. & 865 \\
\hline & O56C02040 & $\mathrm{A}$ & 1999 Apr 22 & G750M & $52 \times .2$ & 6581 & 39.98 & 0. & 600 \\
\hline & O56C02050 & $\mathrm{A}$ & 1999 Apr 22 & G430L & $52 \times .2$ & 4300 & 39.98 & 0. & 600 \\
\hline & O56C02060 & $\mathrm{A}$ & 1999 Apr 22 & G750M & $52 \times .2$ & 6581 & 39.98 & +.3 & 1021 \\
\hline \multirow[t]{2}{*}{ NGC 3516} & O5F406030 & $\mathrm{D}$ & 2000 Jan 18 & G430M & $50 \mathrm{CCD}$ & 5093 & -154.98 & 0. & 2154 \\
\hline & O56C01050 & $\mathrm{A}$ & 2000 Jun 18 & G430L & $52 \times .2$ & 4300 & 38.98 & 0. & 600 \\
\hline \multirow[t]{2}{*}{ NGC 3783} & OBGU03010 & $\mathrm{C}$ & 2011 Mar 23 & G430M & $52 \times .2$ & 4961 & -20.35 & 0. & 696 \\
\hline & OBGU03020 & $\mathrm{C}$ & 2011 Mar 23 & G430M & $52 \times .2$ & 4961 & -20.35 & 0. & 696 \\
\hline \multirow[t]{2}{*}{ NGC 4051} & O5G402010 & $\mathrm{A}$ & 2000 Apr 15 & G430M & $52 \times .2$ & 4961 & 89.78 & -.05 & 1796 \\
\hline & O5G402020 & $\mathrm{A}$ & 2000 Apr 15 & G430M & $52 \times .2$ & 4961 & 89.78 & +.2 & 600 \\
\hline \multirow[t]{2}{*}{ NGC 4303} & O6LC01010 & $\mathrm{A}$ & 2003 Mar 04 & G430M & $52 \times .2$ & 4961 & -140.35 & 0. & 2156 \\
\hline & O6LC01020 & $\mathrm{A}$ & 2003 Mar 04 & G430L & $52 \times .2$ & 4961 & -140.35 & 0. & 1200 \\
\hline \multirow[t]{3}{*}{ NGC 4395} & OBGU04010 & $\mathrm{C}$ & 2011 May 25 & G430M & $52 \times .2$ & 4961 & 64.65 & 0. & 693 \\
\hline & OBGU04020 & $\mathrm{C}$ & 2011 May 25 & G430M & $52 \times .2$ & 4961 & 64.65 & 0. & 693 \\
\hline & OBGU04030 & $\mathrm{C}$ & 2011 May 25 & G430M & $52 \times .2$ & 4961 & 64.65 & 0 . & 693 \\
\hline \multirow[t]{2}{*}{ NGC 4507} & O5DF03010 & $\mathrm{A}$ & 2001 Apr 04 & G430M & $52 \times .2$ & 4961 & -34.35 & 0. & 1440 \\
\hline & O5DF03010 & $\mathrm{A}$ & 2001 Apr 04 & G430L & $52 \times .2$ & 4300 & -34.35 & 0. & 624 \\
\hline NGC 5135 & O6BU07010 & A & 2002 Jan 11 & G750M & $52 \times .2$ & 6581 & -115.81 & 0. & 1080 \\
\hline
\end{tabular}


Table 6.1 Continued from previous page

\begin{tabular}{|c|c|c|c|c|c|c|c|c|c|}
\hline Target & STScI Data Set & Source & $\begin{array}{l}\text { Date } \\
\text { (UT) }\end{array}$ & Grating & Aperture & $\begin{array}{c}\text { Central } \lambda \\
(\AA)\end{array}$ & $\begin{array}{l}\text { P.A. } \\
\text { (deg) }\end{array}$ & $\begin{array}{c}\text { Offset } \\
\text { (arcsec) }\end{array}$ & $\begin{array}{c}\text { Exposure } \\
(\mathrm{s})\end{array}$ \\
\hline & O6BU07020 & A & 2002 Jan 11 & G750M & $52 \times .2$ & 6581 & -115.81 & 0 & 1104 \\
\hline & O6BU07030 & $\mathrm{A}$ & 2002 Jan 11 & G750M & $52 \times .2$ & 6581 & -115.81 & 0 . & 832 \\
\hline & O6BU07040 & A & 2002 Jan 11 & G430L & $52 \times .2$ & 4300 & -115.81 & 0 . & 840 \\
\hline & O6BU07040 & A & 2002 Jan 11 & G430L & $52 \times .2$ & 4300 & -115.81 & 0 . & 829 \\
\hline \multirow{5}{*}{ NGC 5252} & O56C04010 & A & 1999 Jan 27 & G750M & $52 \times .2$ & 6581 & -135.52 & -.2 & 1080 \\
\hline & O56C04030 & A & 1999 Jan 27 & G750M & $52 \times .2$ & 6581 & -135.52 & 0 . & 841 \\
\hline & O56C04040 & A & 1999 Jan 27 & G750M & $52 \times .2$ & 6581 & -135.52 & 0 . & 600 \\
\hline & O56C04050 & $\mathrm{A}$ & 1999 Jan 27 & G430L & $52 \times .2$ & 4300 & -135.52 & 0 . & 600 \\
\hline & O56C04060 & A & 1999 Jan 27 & G750M & $52 \times .2$ & 6581 & -135.52 & +.2 & 997 \\
\hline \multirow[t]{5}{*}{ NGC 5283} & O6BU08010 & $\mathrm{A}$ & 2001 Oct 11 & $\mathrm{G} 750 \mathrm{M}$ & $52 \times .2$ & 6581 & -37.06 & 0. & 1200 \\
\hline & O6BU08020 & A & 2001 Oct 11 & G750M & $52 \times .2$ & 6581 & -37.06 & 0 . & 1213 \\
\hline & O6BU08030 & A & 2001 Oct 11 & G750M & $52 \times .2$ & 6581 & -37.06 & 0 . & 900 \\
\hline & O6BU08040 & A & 2001 Oct 11 & G430L & $52 \times .2$ & 4300 & -37.06 & 0 . & 900 \\
\hline & O6BU08050 & A & 2001 Oct 11 & G430L & $52 \times .2$ & 4300 & -37.06 & 0 . & 900 \\
\hline \multirow[t]{5}{*}{ NGC 5347} & O6BU09010 & $\mathrm{A}$ & 2001 Dec 24 & $\mathrm{G} 750 \mathrm{M}$ & $52 \times .2$ & 6581 & -102.20 & 0 . & 1080 \\
\hline & O6BU09020 & A & 2001 Dec 24 & G750M & $52 \times .2$ & 6581 & -102.20 & 0 . & 1126 \\
\hline & O6BU09030 & A & 2001 Dec 25 & G750M & $52 \times .2$ & 6581 & -102.20 & 0 . & 840 \\
\hline & O6BU09040 & A & 2001 Dec 25 & G430L & $52 \times .2$ & 4300 & -102.20 & 0 . & 840 \\
\hline & O6BU09050 & A & 2001 Dec 25 & G430L & $52 \times .2$ & 4300 & -102.20 & 0 . & 851 \\
\hline \multirow{5}{*}{ NGC 5427} & O6BU10010 & A & 2002 Jan 04 & G750M & $52 \times .2$ & 6581 & -113.38 & 0 . & 1080 \\
\hline & O6BU10020 & A & 2002 Jan 04 & G750M & $52 \times .2$ & 6581 & -113.38 & 0 . & 1080 \\
\hline & O6BU10030 & A & 2002 Jan 04 & G750M & $52 \times .2$ & 6581 & -113.38 & 0 . & 840 \\
\hline & O6BU10040 & A & 2002 Jan 04 & G430L & $52 \times .2$ & 4300 & -113.38 & 0 . & 840 \\
\hline & O6BU10050 & $\mathrm{A}$ & 2002 Jan 04 & G430L & $52 \times .2$ & 4300 & -113.38 & 0 . & 805 \\
\hline NGC 5506 & O5F407030 & D & 2000 Mar 18 & G430M & 50CCD & 4961 & -153.39 & 0 . & 2096 \\
\hline \multirow[t]{3}{*}{ NGC 5548} & OBGU06010 & $\mathrm{C}$ & 2011 Mar 11 & G430M & $52 \times .2$ & 5093 & -160.35 & 0 . & 695 \\
\hline & OBGU06020 & $\mathrm{C}$ & 2011 Mar 11 & G430M & $52 \times .2$ & 5093 & -160.35 & 0 . & 695 \\
\hline & OBGU06030 & $\mathrm{C}$ & 2011 Mar 11 & G430M & $52 \times .2$ & 5093 & -160.35 & 0 . & 695 \\
\hline \multirow[t]{6}{*}{ NGC 5643} & O5F408030 & $\mathrm{D}$ & 2000 Feb 23 & G430M & $50 \mathrm{CCD}$ & 4961 & -99.98 & 0 . & 2107 \\
\hline & O6BU11010 & $\mathrm{A}$ & 2000 Mar 12 & G750M & $52 \times .2$ & 6581 & -128.04 & 0 . & 1140 \\
\hline & O6BU11020 & $\mathrm{A}$ & 2000 Mar 12 & G750M & $52 \times .2$ & 6581 & -128.04 & 0 . & 1223 \\
\hline & O6BU11030 & $\mathrm{A}$ & 2000 Mar 12 & G750M & $52 \times .2$ & 6581 & -128.04 & 0 . & 900 \\
\hline & O6BU11040 & $\mathrm{A}$ & 2000 Mar 12 & G430L & $52 \times .2$ & 4300 & -128.04 & 0. & 840 \\
\hline & O6BU11050 & A & 2000 Mar 12 & G430L & $52 \times .2$ & 4300 & -128.04 & 0. & 868 \\
\hline NGC 5695 & O6BU12010 & A & 2001 Aug 11 & G750M & $52 \times .2$ & 6581 & 50.65 & 0 . & 1080 \\
\hline
\end{tabular}


Table 6.1 Continued from previous page

\begin{tabular}{|c|c|c|c|c|c|c|c|c|c|}
\hline Target & STScI Data Set & Source & $\begin{array}{l}\text { Date } \\
\text { (UT) }\end{array}$ & Grating & Aperture & $\begin{array}{c}\text { Central } \lambda \\
(\AA)\end{array}$ & $\begin{array}{l}\text { P.A. } \\
\text { (deg) }\end{array}$ & $\begin{array}{l}\text { Offset } \\
\text { (arcsec) }\end{array}$ & $\begin{array}{c}\text { Exposure } \\
\text { (s) }\end{array}$ \\
\hline & O6BU12020 & $\mathrm{A}$ & 2001 Aug 11 & G750M & $52 \times .2$ & 6581 & 50.65 & 0 & 1158 \\
\hline & O6BU12030 & $\mathrm{A}$ & $2001 \mathrm{Aug} 11$ & G750M & $52 \times .2$ & 6581 & 50.65 & 0. & 900 \\
\hline & O6BU12040 & A & 2001 Aug 11 & G430L & $52 \times .2$ & 4300 & 50.65 & 0 . & 840 \\
\hline & O6BU12050 & A & 2001 Aug 11 & G430L & $52 \times .2$ & 4300 & 50.65 & 0 . & 823 \\
\hline NGC 5728 & O5F409030 & D & 2000 Apr 24 & G430M & $50 \mathrm{CCD}$ & 5093 & -79.98 & 0 . & 2110 \\
\hline NGC 5929 & O5G403010 & A & 2000 Feb 07 & G430M & $52 \times .2$ & 4961 & -134.62 & 0. & 1524 \\
\hline \multirow[t]{5}{*}{ NGC 6300} & O6BU13010 & A & 2001 Nov 08 & G750M & $52 \times .2$ & 6581 & 90.28 & 0. & 1140 \\
\hline & O6BU13020 & A & 2001 Nov 08 & G750M & $52 \times .2$ & 6581 & 90.28 & 0 . & 1226 \\
\hline & O6BU13030 & A & 2001 Nov 08 & G750M & $52 \times .2$ & 6581 & 90.28 & 0. & 840 \\
\hline & O6BU13040 & A & 2001 Nov 08 & G430L & $52 \times .2$ & 4300 & 90.28 & 0. & 900 \\
\hline & O6BU13050 & A & 2001 Nov 08 & G430L & $52 \times .2$ & 4300 & 90.28 & 0 . & 951 \\
\hline NGC 7212 & O5F410030 & $\mathrm{D}$ & 2000 Jun 04 & G430M & 50CCD & 5093 & -174.98 & 0 . & 2112 \\
\hline \multirow[t]{3}{*}{ NGC 7469} & OBGU09010 & $\mathrm{C}$ & 2010 Oct 05 & G430M & $52 \times .1$ & 5093 & 34.65 & 0. & 697 \\
\hline & OBGU09020 & $\mathrm{C}$ & 2010 Oct 05 & G430M & $52 \times .1$ & 5093 & 34.65 & 0. & 697 \\
\hline & OBGU09030 & $\mathrm{C}$ & 2010 Oct 05 & G430M & $52 \times .1$ & 5093 & 34.65 & 0. & 697 \\
\hline \multirow[t]{2}{*}{ NGC 7674} & O5DF04010 & A & 2000 Sep 12 & G430M & $52 \times .2$ & 5093 & 124.65 & 0 . & 1140 \\
\hline & O5DF04020 & A & 2000 Sep 12 & G430L & $52 \times .2$ & 4300 & 124.65 & 0. & 600 \\
\hline \multirow[t]{5}{*}{ NGC 7682} & O6BU14010 & A & 2001 Oct 23 & G750M & $52 \times .2$ & 6581 & 17.85 & 0. & 1080 \\
\hline & O6BU14020 & A & 2001 Oct 23 & G750M & $52 \times .2$ & 6581 & 17.85 & 0 . & 1080 \\
\hline & O6BU14030 & A & 2001 Oct 23 & G750M & $52 \times .2$ & 6581 & 17.85 & 0. & 840 \\
\hline & O6BU14040 & A & 2001 Oct 23 & G430L & $52 \times .2$ & 4300 & 17.85 & 0. & 840 \\
\hline & O6BU14050 & A & 2001 Oct 23 & G430L & $52 \times .2$ & 4300 & 17.85 & 0. & 805 \\
\hline \multirow{5}{*}{ NGC 788} & O6BU15010 & A & 2001 Sep 17 & G750M & $52 \times .2$ & 6581 & -129.56 & 0 . & 1080 \\
\hline & O6BU15020 & A & 2001 Sep 17 & G750M & $52 \times .2$ & 6581 & -129.56 & 0. & 1080 \\
\hline & O6BU15030 & A & 2001 Sep 17 & G750M & $52 \times .2$ & 6581 & -129.56 & 0. & 840 \\
\hline & O6BU15040 & A & 2001 Sep 17 & G430L & $52 \times .2$ & 4300 & -129.56 & 0 . & 840 \\
\hline & O6BU15050 & A & 2001 Sep 17 & G430L & $52 \times .2$ & 4300 & -129.56 & 0. & 805 \\
\hline \multirow[t]{5}{*}{ UM 146} & O6BU16010 & A & 2001 Dec 20 & G750M & $52 \times .2$ & 6581 & 21.33 & 0. & 1080 \\
\hline & O6BU16020 & A & 2001 Dec 20 & G750M & $52 \times .2$ & 6581 & 21.33 & 0. & 1080 \\
\hline & O6BU16030 & A & 2001 Dec 20 & G750M & $52 \times .2$ & 6581 & 21.33 & 0 . & 840 \\
\hline & O6BU16040 & A & 2001 Dec 20 & G430L & $52 \times .2$ & 4300 & 21.33 & 0. & 840 \\
\hline & O6BU16050 & A & 2001 Dec 20 & G430L & $52 \times .2$ & 4300 & 21.33 & 0. & 805 \\
\hline
\end{tabular}

a'Sources: (A) MAST Archive (B) HST Prop ID: 11611 (C) HST Prop ID: 12212 (D) Ruiz et al. (2005) 


\subsubsection{Imaging}

Table 6.2 lists the HST instruments used to image each AGN, as well as their redshifts and corresponding distances and transverse scales assuming $\mathrm{H}_{o}=73 \mathrm{~km} \mathrm{Mpc} \mathrm{s}{ }^{-1}$. Images with overplotted STIS slit positions are depicted in Appendix A. Images taken with filters containing [O III] $\lambda 5007$ or H $\alpha \lambda 6563$ emission were prefered, otherwise wide-band continuum filters were used. All redshifts were taken from the NASA/IPAC Extragalactic Database (NED).

\subsection{Analysis}

Appendix C shows radial velocities, FWHM, and fluxes for each observation of the expanded sample as a function of projected distance from the nucleus using the line analysis procedure described in Section 2.2. Each figure depicts lines measured using G430L, G430M, or G750M gratings with green stars, blue triangles, and red diamonds respectively. Kinematic classifications for our entire sample, as described below, are listed in Table 6.3. From the expanded sample, we found only 12 additional AGN that clearly show distinct asymmetrical red/blue shifts in their [O III] kinematics that are characteristic of biconical outflows seen in our previously modeled targets. The resultant models for each of these AGN are detailed in Section 6.3, with the final parameter values of each modeled AGN, including the five previously observed AGN, listed in Table 6.4. When comparing properties of our 17 successfully versus the remaining unsuccessfully modeled AGN, we find no bias in the ability to model the kinematics due to Seyfert type (Figure 6.1) or distance from Earth (Figure 6.2)

Of the other targets, 17 show kinematics that we have deemed 'Ambiguous'. Targets with ambiguous kinematics have a symmetrical component on each side of the nucleus traveling in opposite directions, similar to only seeing the two, near-systemic velocity components of Mrk 573. These components possibly correspond to parallel sides of a half of the bicone, 
Table 6.2 Expanded Sample: HST Imaging Observations and Redshifts

\begin{tabular}{|c|c|c|c|c|c|c|c|}
\hline Target & $\bar{Z}$ & Source & $\begin{array}{c}\text { Dist. } \\
\text { (Mpc) }\end{array}$ & $\begin{array}{c}\text { Scale } \\
\left(\mathrm{pc} /{ }^{\prime \prime}\right)\end{array}$ & Type & Instr. & Filter \\
\hline Akn 564 & 0.024684 & $21 \mathrm{~cm}$ & 101.37 & 491.46 & 1 & WFC3 & FQ508N \\
\hline Circinus & 0.001453 & $21 \mathrm{~cm}$ & 5.96 & 28.92 & 2 & WFPC2 & F606W \\
\hline IC 3639 & 0.010924 & $21 \mathrm{~cm}$ & 44.86 & 217.49 & 2 & WFPC2 & F606W \\
\hline IRAS 11058-1131 & 0.054828 & Stellar & 225.16 & 1091.63 & 2 & WFPC2 & $\mathrm{F} 547 \mathrm{M}$ \\
\hline MCG-6-30-15 & 0.007749 & Stellar & 31.82 & 154.28 & 1 & WFPC2 & FR533N \\
\hline Mrk 34 & 0.051167 & Stellar & 210.13 & 1018.74 & 2 & WFPC2 & FR533N \\
\hline Mrk 279 & 0.030451 & Stellar & 125.05 & 606.28 & 1 & WFC3 & FQ508N \\
\hline Mrk 348 & 0.015034 & $21 \mathrm{~cm}$ & 61.74 & 299.32 & 2 & WFPC2 & FR533N \\
\hline Mrk 463e & 0.050000 & - & 205.33 & 995.50 & 2 & WFPC2 & FR533N \\
\hline Mrk 493 & 0.031485 & $21 \mathrm{~cm}$ & 129.30 & 626.86 & 1 & WFPC2 & F606W \\
\hline Mrk 509 & 0.034397 & Stellar & 141.26 & 684.84 & 1 & WFC3 & FQ508N \\
\hline Mrk 705 & 0.029150 & Stellar & 119.71 & 580.37 & 1 & WFPC2 & FR533N \\
\hline Mrk 766 & 0.012929 & Stellar & 53.09 & 257.41 & 1 & WFPC2 & FR533N \\
\hline Mrk 1040 & 0.016642 & $21 \mathrm{~cm}$ & 68.34 & 331.34 & 1 & WFPC2 & FR533N \\
\hline Mrk 1066 & 0.011858 & Stellar & 48.69 & 236.09 & 2 & $\mathrm{WF} / \mathrm{PC}$ & $\mathrm{F} 492 \mathrm{M}$ \\
\hline NGC 1358 & 0.013436 & $21 \mathrm{~cm}$ & 55.17 & 267.51 & 2 & WFPC2 & F606W \\
\hline NGC 1386 & 0.002895 & Stellar & 11.88 & 57.63 & 2 & WFPC2 & F502N \\
\hline NGC 1667 & 0.015257 & $21 \mathrm{~cm}$ & 62.65 & 303.76 & 2 & WFPC2 & F606W \\
\hline NGC 2110 & 0.007789 & Stellar & 31.98 & 155.07 & 2 & WFPC2 & F606W \\
\hline NGC 2273 & 0.006138 & $21 \mathrm{~cm}$ & 25.20 & 122.20 & $\overline{2}$ & WFPC2 & FR533N \\
\hline NGC 3081 & 0.007988 & $21 \mathrm{~cm}$ & 32.80 & 159.04 & 2 & WFPC2 & FR533N \\
\hline NGC 3227 & 0.003859 & $21 \mathrm{~cm}$ & 15.84 & 76.83 & 1 & WFPC2 & F606W \\
\hline NGC 3393 & 0.012509 & $21 \mathrm{~cm}$ & 51.37 & 249.05 & 2 & WFC3 & FQ508N \\
\hline NGC 3516 & 0.008836 & Stellar & 36.28 & 175.92 & 1 & WFPC2 & FR533N \\
\hline NGC 3783 & 0.009730 & $21 \mathrm{~cm}$ & 39.95 & 193.72 & 1 & WFPC2 & FR533N \\
\hline NGC 4051 & 0.002418 & $21 \mathrm{~cm}$ & 9.93 & 48.14 & 1 & WFC3 & $\mathrm{F} 502 \mathrm{~N}$ \\
\hline NGC 4303 & 0.005234 & $21 \mathrm{~cm}$ & 21.49 & 104.20 & 2 & WFPC2 & F606W \\
\hline NGC 4395 & 0.001064 & $21 \mathrm{~cm}$ & 4.36 & 21.18 & 1 & WFC3 & F502N \\
\hline NGC 4507 & 0.011829 & $21 \mathrm{~cm}$ & 48.57 & 235.51 & 2 & WFPC2 & FR533N \\
\hline NGC 5135 & 0.013693 & $21 \mathrm{~cm}$ & 56.23 & 272.62 & 2 & WFPC2 & F606W \\
\hline NGC 5252 & 0.023093 & $21 \mathrm{~cm}$ & 94.83 & 459.78 & 1.9 & WFPC2 & FR533N \\
\hline NGC 5283 & 0.010404 & Stellar & 42.72 & 207.14 & 2 & WFPC2 & F606W \\
\hline NGC 5347 & 0.007959 & $21 \mathrm{~cm}$ & 32.68 & 158.46 & 2 & WFPC2 & FR533N \\
\hline NGC 5427 & 0.008733 & $21 \mathrm{~cm}$ & 35.86 & 173.87 & 2 & WFPC2 & F606W \\
\hline NGC 5506 & 0.006084 & $21 \mathrm{~cm}$ & 24.98 & 121.13 & 2 & $\mathrm{FOC}$ & F501N \\
\hline NGC 5548 & 0.017175 & $21 \mathrm{~cm}$ & 70.53 & 341.95 & 1 & WFC3 & FQ508N \\
\hline NGC 5643 & 0.003999 & $21 \mathrm{~cm}$ & 16.42 & 79.62 & 2 & WFPC2 & F502N \\
\hline NGC 5695 & 0.014093 & $21 \mathrm{~cm}$ & 57.87 & 280.59 & 2 & WFPC2 & F606W \\
\hline NGC 5728 & 0.009316 & $21 \mathrm{~cm}$ & 38.25 & 185.48 & 2 & $\mathrm{WF} / \mathrm{PC}$ & F492M \\
\hline NGC 5929 & 0.008543 & $21 \mathrm{~cm}$ & 35.08 & 170.09 & 2 & WFPC2 & F606W \\
\hline NGC 6300 & 0.003699 & $21 \mathrm{~cm}$ & 15.19 & 73.64 & 2 & WFPC2 & F606W \\
\hline NGC 7212 & 0.026001 & Stellar & 106.78 & 517.68 & 2 & WFPC2 & FR533N \\
\hline NGC 7469 & 0.016268 & $21 \mathrm{~cm}$ & 66.80 & 323.89 & 1 & WFPC2 & FR533N \\
\hline NGC 7674 & 0.029030 & $21 \mathrm{~cm}$ & 119.21 & 577.98 & 2 & WFPC2 & FR533P15 \\
\hline NGC 7682 & 0.017140 & $21 \mathrm{~cm}$ & 70.38 & 341.25 & 2 & WFPC2 & F606W \\
\hline NGC 788 & 0.013603 & Stellar & 55.86 & 270.83 & 2 & WFPC2 & F606W \\
\hline UM 146 & 0.017225 & $21 \mathrm{~cm}$ & 70.73 & 342.95 & 1.9 & WFPC2 & F606W \\
\hline
\end{tabular}




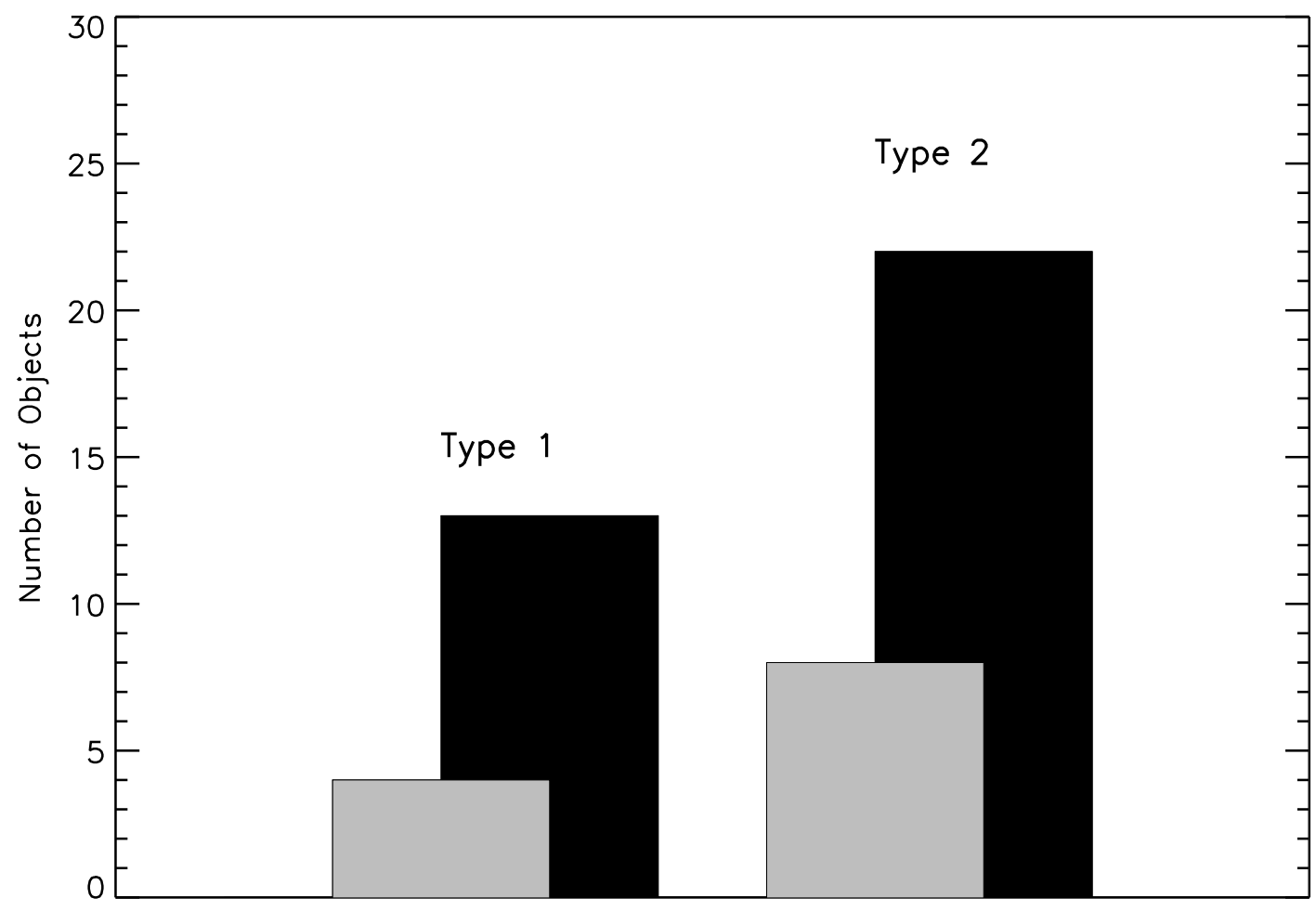

Figure 6.1 Histogram displaying the distribution of Seyfert type for AGN with modeled (grey) and unmodeled (black) kinematics in our sample. The fractions of modeled to unmodeled Seyfert 1s and Seyfert 2s appear to be approximately proportionate.

though, as many do not decelerate after a certain distance, they could also be due to rotation or some other factor not incorporated into our current model. Even with confirmation that the kinematics were half of an outflowing system, we would be unable to create a successful kinematic model as the components are not adjacent to one another (as mentioned in Section $3.4)$.

Six additional targets show kinematics that reside near systemic velocity, possibly containing large gradients in velocity similar to those caused by in situ acceleration in Mrk 573. These knotty, turbulent NLRs are too complex to identify any kinematic components within the data, and are thus identified as 'Complex' kinematics targets. 


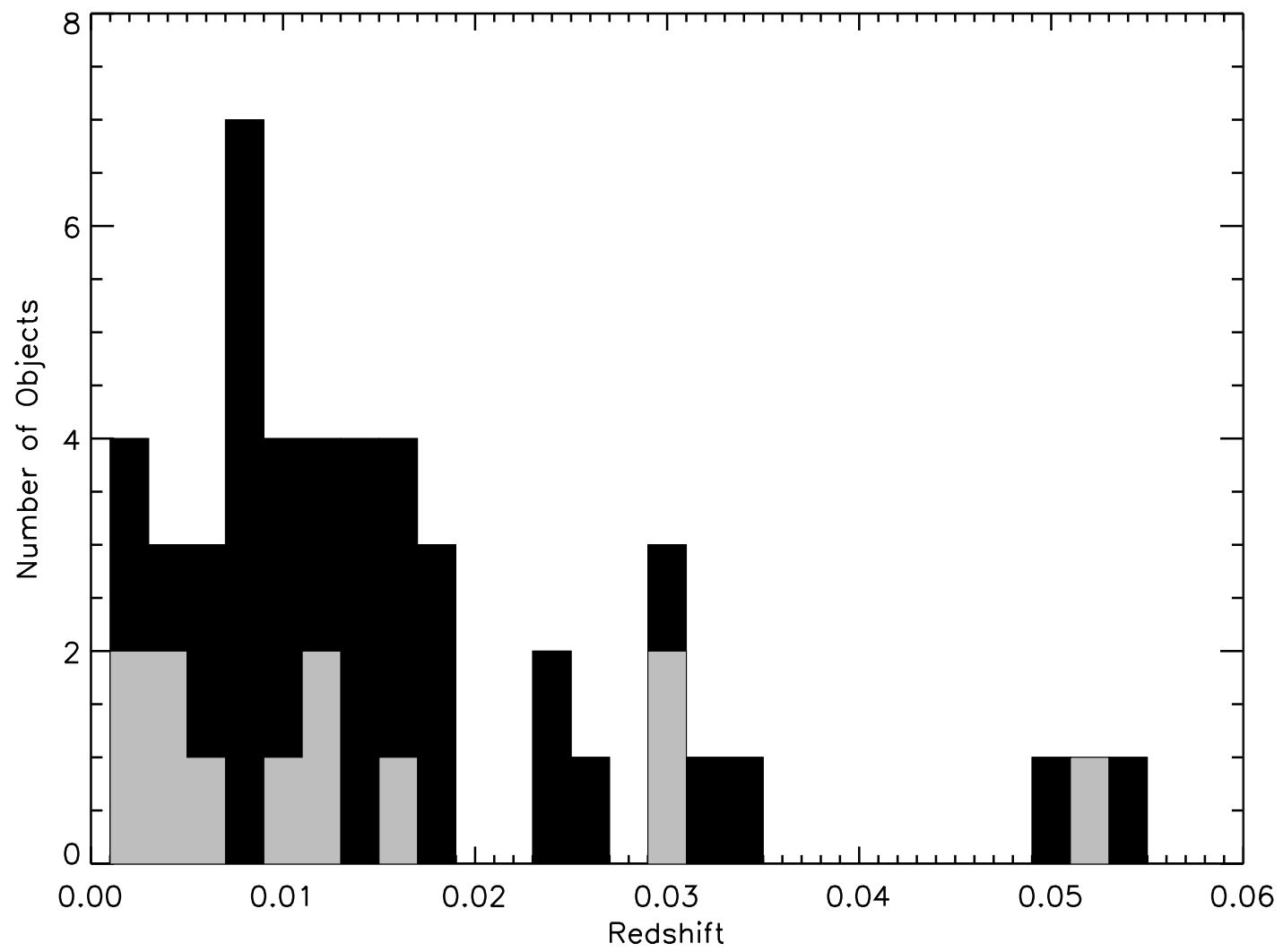

Figure 6.2 Histogram displaying the distribution of redshifts for AGN with modeled (grey) and unmodeled (black) kinematics in our sample. AGN with modeled and unmodeled kinematics appear to be evenly distributed.

There are 9 targets that do not contain enough data points in their kinematic plots to fit a model, and their kinematics are simply defined as 'Compact'. Some of these contain highly blueshifted velocities near the nucleus, suggesting outflow, but they are not resolvable into individual components. Therefore, it is unclear what portion of the NLR we are viewing, e.g., whether we are looking at the edge of an intermediately inclined cone or possibly an entire blueshifted cone.

Finally, the remaining 3 targets cannot be modeled as their observations do not detect the NLR. Two targets, Mrk 348 and NGC 5347, were observed with the slit position outside the NLR, thus only detecting the nucleus emission. Observations for NGC 4303 contain only 
Table 6.3 Total sample AGN classifications

\begin{tabular}{c|ccc}
\hline Outflow & Circinus & Mrk 3 & Mrk 34 \\
& Mrk 78 & Mrk 279 & Mrk 573 \\
& Mrk 1066 & NGC 1068 & NGC 1667 \\
& NGC 3227 & NGC 3783 & NGC 4051 \\
& NGC 4151 & NGC 4507 & NGC 5506 \\
& NGC 5643 & NGC 7674 & \\
\hline Ambiguous & Akn 564 & IC 3639 & MCG-6-30-15 \\
& Mrk 493 & Mrk 509 & NGC 1358 \\
& NGC 2110 & NGC 2273 & NGC 3516 \\
& NGC 4395 & NGC 5252 & NGC 5283 \\
& NGC 5728 & NGC 5929 & NGC 7682 \\
& NGC 788 & UM 146 & \\
\hline Complex & IRAS 11058-1131 & NGC 1386 & NGC 3081 \\
& NGC 3393 & NGC 5135 & NGC 7212 \\
\hline Compact & Mrk 463e & Mrk 705 & Mrk 766 \\
& Mrk 1040 & NGC 5427 & NGC 5548 \\
& NGC 5695 & NGC 6300 & NGC 7469 \\
\hline Poor & Mrk 348 & NGC 4303 & NGC 5347 \\
& & & \\
\hline
\end{tabular}

continuum emission and no [O III] lines are clearly present.

\subsection{Targets With Clear Outflow Signatures}

Table 6.4 contains the full set of kinematic model parameters of the twelve AGN with clear biconical outflow signatures from the expanded sample as well as the five previously modeled AGN (Mrk 3, Mrk 78, Mrk 573, NGC 1068, and NGC 4151). The table also gives position angles and inclinations of each host galaxy disk required for our geometric models.

\subsubsection{Circinus}

Figure A.1 shows that this is a Seyfert 2 galaxy with a single NLR cone visible in [O III] imaging near perpendicular to the major axis of the host disk. As shown in Figures 6.3, D.1 and D.2, the modeled kinematics provide a good fit to the data, matching well to both visible 

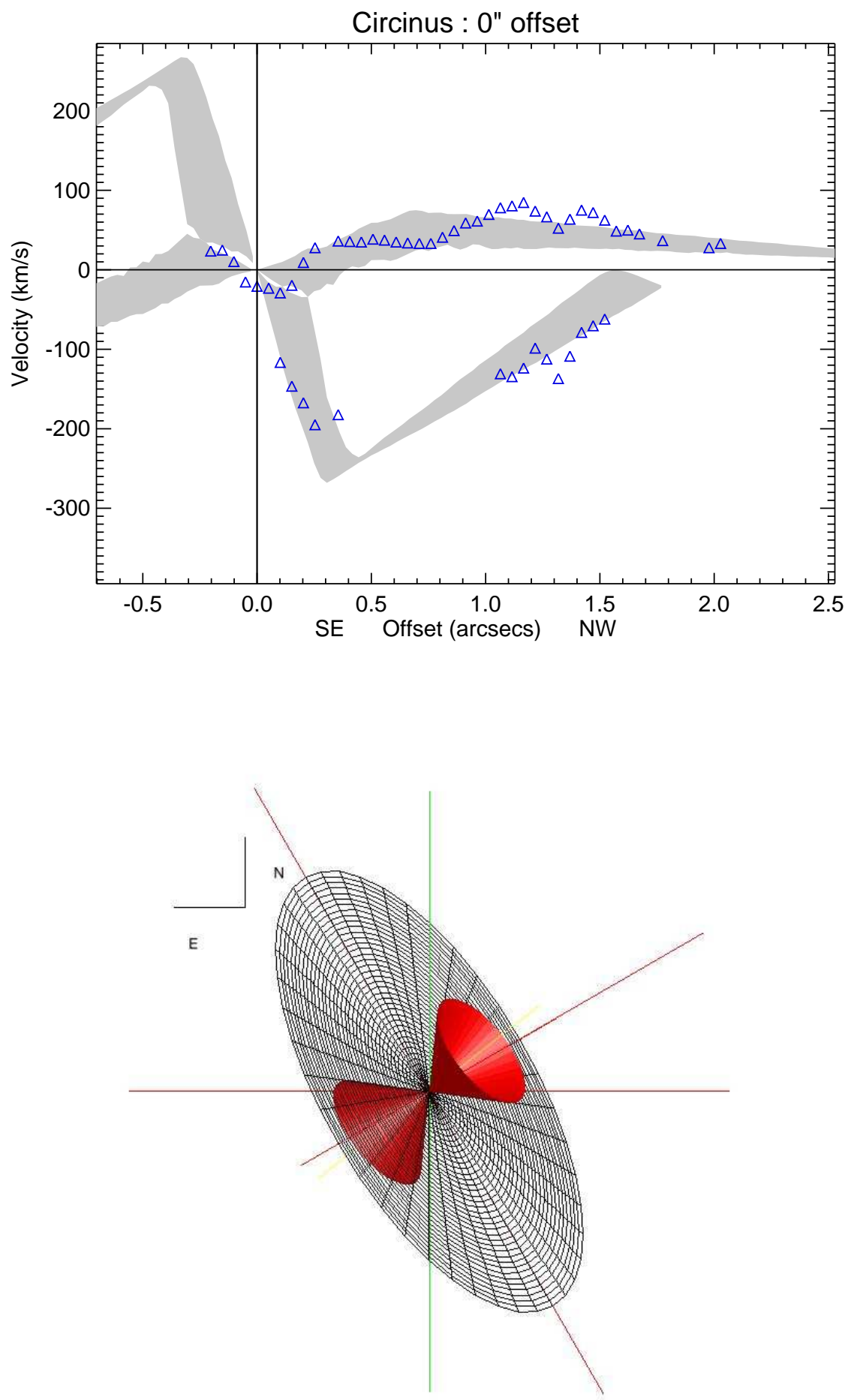

Figure 6.3 Above: Kinematic model for the central slit position of Circinus. Additional models for adjacent slits are shown in Appendix D. Below: Corresponding geometric model of the NLR with disk geometry. 
Table 6.4 Total Sample Modeled AGN Parameters. Inclination direction specifies which end of the NLR bicone is inclined out of the plane of the sky toward Earth.

References: (1) Freeman et al. (1977), (2) Schmitt \& Kinney (2000), (3) Kinney et al. (2000), (4) NED, (5) Xilouris \& Papadakis (2002), (6) Hicks et al. (2009), (7) Das et al. (2005), (8) Das et al. (2006), (9) Crenshaw et al. (2010a)

\begin{tabular}{|c|c|c|c|c|c|c|c|c|c|c|}
\hline \multirow[t]{2}{*}{ Target } & \multicolumn{7}{|c|}{ NLR Bicone } & \multicolumn{3}{|c|}{ Host Disk } \\
\hline & $\begin{array}{c}P . A . \\
\left.{ }^{\circ}\right)\end{array}$ & $\begin{array}{c}i \\
\left(^{\circ}\right)\end{array}$ & $\begin{array}{c}\theta_{\min } \\
\left(^{\circ}\right)\end{array}$ & $\begin{array}{c}\theta_{\max } \\
\left({ }^{\circ}\right)\end{array}$ & $\begin{array}{c}v_{\max } \\
(\mathrm{km} / \mathrm{s})\end{array}$ & $\begin{array}{l}z_{\max } \\
(\mathrm{pc})\end{array}$ & $\begin{array}{c}r_{t} \\
(\mathrm{pc})\end{array}$ & $\begin{array}{c}\text { P.A. } \\
\left(^{\circ}\right)\end{array}$ & $\begin{array}{c}i \\
\left(^{\circ}\right)\end{array}$ & $\begin{array}{l}\text { Disk } \\
\text { Ref. }\end{array}$ \\
\hline Circinus & -52 & $25(\mathrm{NW})$ & 36 & 41 & 300 & 50 & 13 & 30 & 65 & 1 \\
\hline Mrk 3 & 71 & $05(\mathrm{NE})$ & - & 51 & 800 & 270 & 80 & 129 & 64 & 9 \\
\hline Mrk 34 & -32 & 25 (SE) & 30 & 40 & 1500 & 1750 & 1000 & 65 & 30 & 2 \\
\hline Mrk 78 & 65 & $30(\mathrm{SW})$ & 10 & 35 & 1200 & 3200 & 700 & 84 & 55 & 2 \\
\hline Mrk 279 & -24 & $55(\mathrm{SE})$ & 59 & 62 & 1800 & 300 & 250 & 33 & 56 & 3,4 \\
\hline Mrk 573 & -36 & $30(\mathrm{NW})$ & 51 & 53 & 400 & 1200 & 800 & 103 & 30 & 3,2 \\
\hline Mrk 1066 & -41 & $10(\mathrm{NW})$ & 15 & 25 & 900 & 400 & 80 & 90 & 54 & 3 \\
\hline NGC 1068 & 30 & $05(\mathrm{NE})$ & 20 & 40 & 2000 & 400 & 140 & 115 & 28 & 8 \\
\hline NGC 1667 & 55 & $18(\mathrm{NW})$ & 45 & 58 & 300 & 100 & 60 & 5 & 39 & 4 \\
\hline NGC 3227 & 30 & $75(\mathrm{SW})$ & 40 & 55 & 500 & 120 & 60 & -31 & 63 & 2,5 \\
\hline NGC 3783 & -20 & 75 (SE) & 45 & 55 & 130 & 175 & 50 & -15 & 35 & 6 \\
\hline NGC 4051 & 80 & $78(\mathrm{NE})$ & 10 & 25 & 550 & 100 & 30 & 50 & 05 & 6 \\
\hline NGC 4151 & 60 & 45 (SW) & 15 & 33 & 800 & 400 & 96 & 33 & 20 & 7 \\
\hline NGC 4507 & -37 & 43 (NW) & 30 & 50 & 1000 & 200 & 90 & 65 & 28 & 2 \\
\hline NGC 5506 & 22 & $10(\mathrm{SW})$ & 10 & 40 & 550 & 250 & 75 & -89 & 76 & 3 \\
\hline NGC 5643 & 80 & $25(\mathrm{SE})$ & 50 & 55 & 500 & 400 & 100 & 136 & 30 & 3 \\
\hline NGC 7674 & -63 & $30(\mathrm{NW})$ & 35 & 40 & 1000 & 700 & 200 & 76 & 40 & 2 \\
\hline
\end{tabular}

outflow components. At this orientation, it is unlikely that an intersection exists between the extended host disk and the NLR. As the projected opening angle of the model NLR $\left(91^{\circ}\right)$ is close to that of the opening angle seen in available imaging $\left(96^{\circ}\right)$ and the size of the NLR in this very nearby AGN is only $\sim 50 \mathrm{pc}$, it is likely that the NLR is ionizing a dense medium in the galactic disk. The south-east cone is undetectable at optical wavelengths as it is hidden behind the heavy extinction of the galactic disk (Ruiz et al. 2000). Extended filimentary [O III] emission $>10^{\prime \prime}$ from the nucleus is not included in our model as slit positions containing this emission are displaced several arcseconds away from the nucleus, and the remote emission would have been difficult to accurately incorporate into a biconical 
model. An available G750M long-slit spectrum perpendicular to the NLR runs along the major axis and detects $\mathrm{H} \alpha$ emission from H II regions surrounding the nucleus in the plane of the disk (Wilson et al. 2000), peaking at an observed velocity of $\sim 175 \mathrm{~km} \mathrm{~s}^{-1}$. This allows us to determine that the rotation of the host disk, blueshifted to the north and redshifted to the south, combined with NLR imaging confirms that the south-east side of the host disk is closer to us, obscuring the south-east NLR cone. Greenhill et al. (2003) found that $\mathrm{H}_{2} \mathrm{O}$ maser emission traces a warped, edge-on accretion disk at radii between $\sim .1$ and $.4 \mathrm{pc}$ and suggest that the warping of the accretion disk collimates the NLR outflow. The resultant model from their hypothesis is similar to our own, though their full opening angle appears to be over $130^{\circ}$, much larger than our $82^{\circ}$. Slit positions and corresponding spectral images of Circinus STIS obserations are available in Figures A.1, B.1 and B.2.

\subsubsection{Mrk 34}

This is a Seyfert 2 galaxy with a backwards S-shape NLR, similar to Mrk 3 as shown in Figure A.2. The modeled kinematics provide a good fit to the data, matching well to all four components save for a few high-velocity points. Employing our geometric model, we see that the S-shaped formation is likely an intersection between a gas spiral in the host disk and the ionization bicone as the projected area of the host disk enclosed by the outer opening angle matches well with emission seen in the [O III] imaging. Similar to Mrk 573, we believe rotation does not play a role in the kinematics we observe as the low velocity components would correspond to a disk rotating clockwise while the host disk spiral arms are winding up in the counterclockwise direction. Kondratko et al. (2006) identified water maser emission in Mrk 34 due to an edge-on accretion disk. Thus, because our model gives the inclination of the bicone axis to be $25^{\circ}$ out of the plane of the sky, it is possible that Mrk 34 experiences the same warped disk scenario as Circinus. Slit positions and corresponding spectral images of Mrk 34 STIS observations are available in Figures A.2 and B.3. 

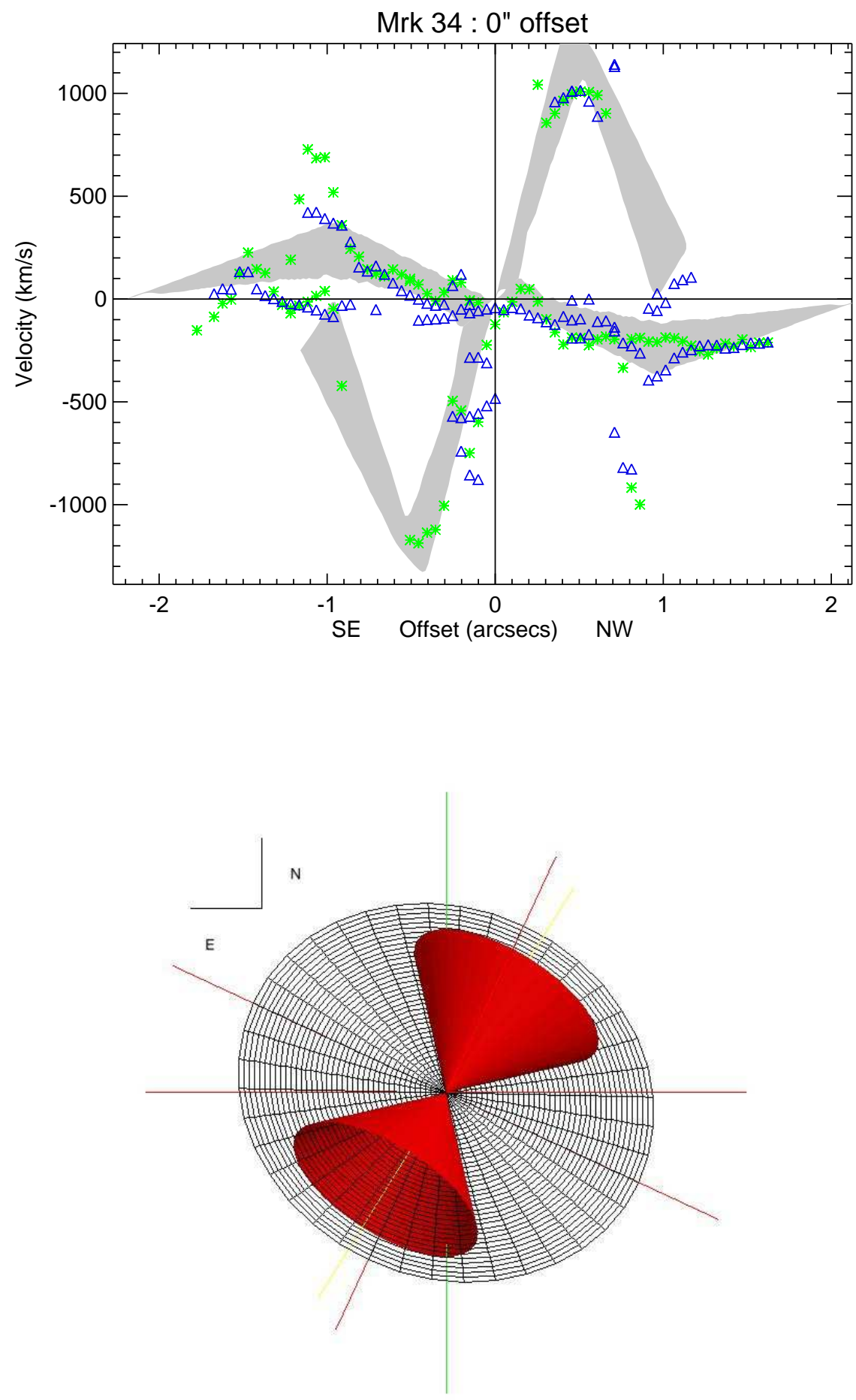

Figure 6.4 Above: Kinematic model for the central slit position of Mrk 34. STIS [O III] observations using the G430M and G430L gratings are shown as blue triangles and green stars respectively. Additional models for adjacent slits shown in Appendix D. Below: Corresponding geometric model of the NLR with disk geometry. 


\subsubsection{Mrk 279}

Mrk 279 is a Seyfert 1 residing in a moderately inclined host. The modeled kinematics provide a good fit to the data, as we are able to account for three components within a small area over the nucleus. Imaging of the NLR shows a compact area slightly more than $1^{\prime \prime}$ in diameter. The opening angle seen in the [O III] imaging has a similar width of $140^{\circ}$ as the intersection between the host disk and NLR in the geometric model, though this orientation results in a bisection of the NLR ionization cone by the galactic disk. This creates a discrepancy between the model and imaging data as we should only see a ' $\mathrm{V}$ ' of emission across the disk without seeing the central portion illuminated because our model assumes the center of the cone is hollow. Slit positions and corresponding spectral images of Mrk 279 STIS obserations are available in Figures A.2 and B.3.

\subsubsection{Mrk 1066}

Mrk 1066 hosts the Seyfert 2 AGN with the smallest opening angle in our sample with a $\theta_{\max }$ of $25^{\circ}$. The modeled kinematics provide a good fit to the data. The majority of the detected emission resides in three knots northwest of the nucleus, which can be modeled as a single cone inclined $10^{\circ}$ out of the plane of the sky. Bower et al. (1995) creates an [O III $]+\mathrm{H} \beta$ emission-line image that depicts a similar single cone to the northwest of the AGN nucleus with a $\theta_{\max }$ of $23^{\circ}$. At the modeled orientation, including the disk geometry determined by Kinney et al. (2000), the bicone does not intersect with the host disk and obscures the unobserved second cone, similar to Circinus. Likewise, the close proximity between the projected model opening angle $\left(51^{\circ}\right)$ and the opening angle seen in available imaging $\left(47^{\circ}\right)$ suggests that the NLR is ionizing a large medium above the host disk. Slit positions and corresponding spectral images of Mrk 1066 STIS obserations are available in Figures A.2 and B.3. 

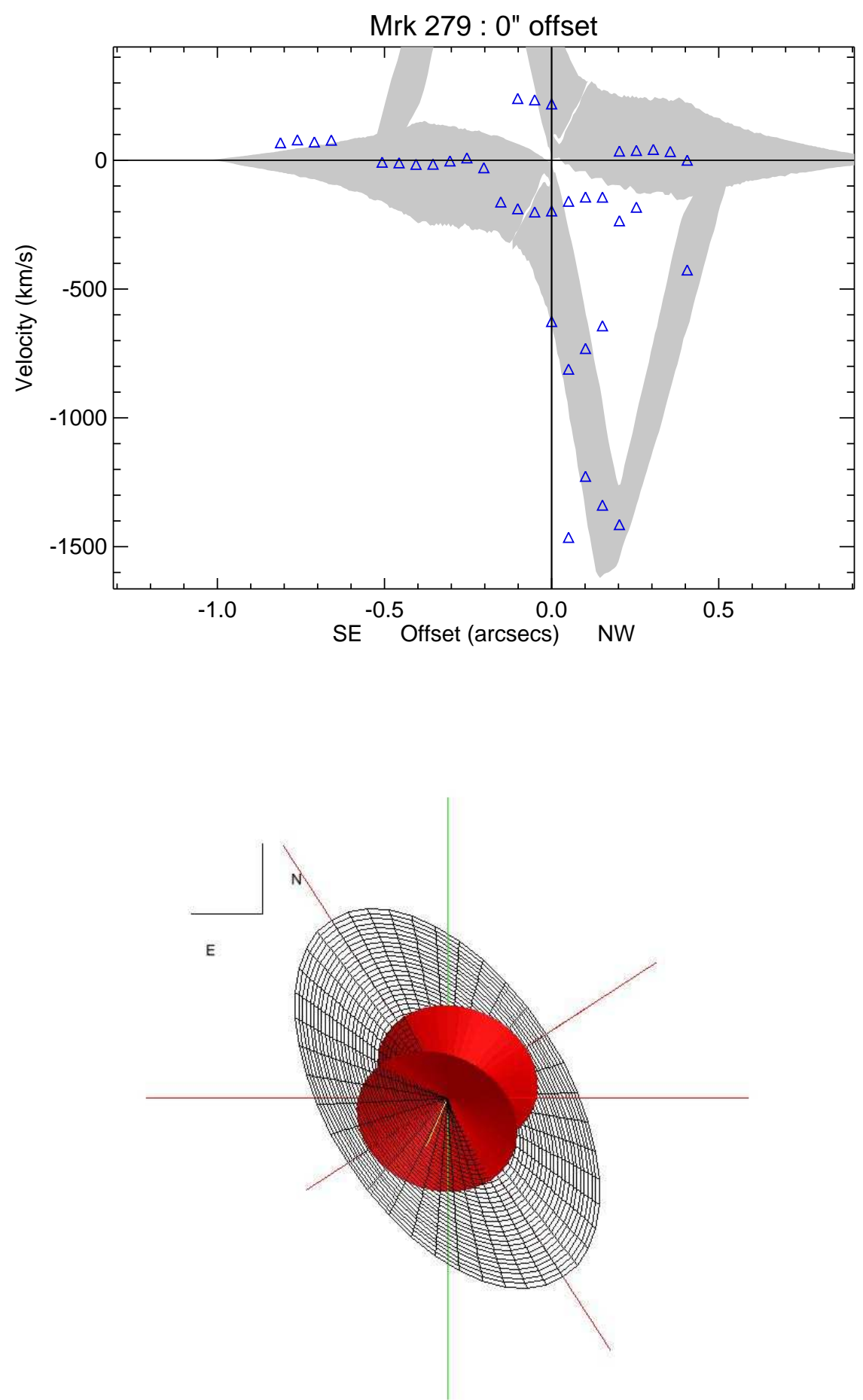

Figure 6.5 Above: Kinematic model of Mrk 279. Below: Corresponding geometric model of the NLR with disk geometry. 

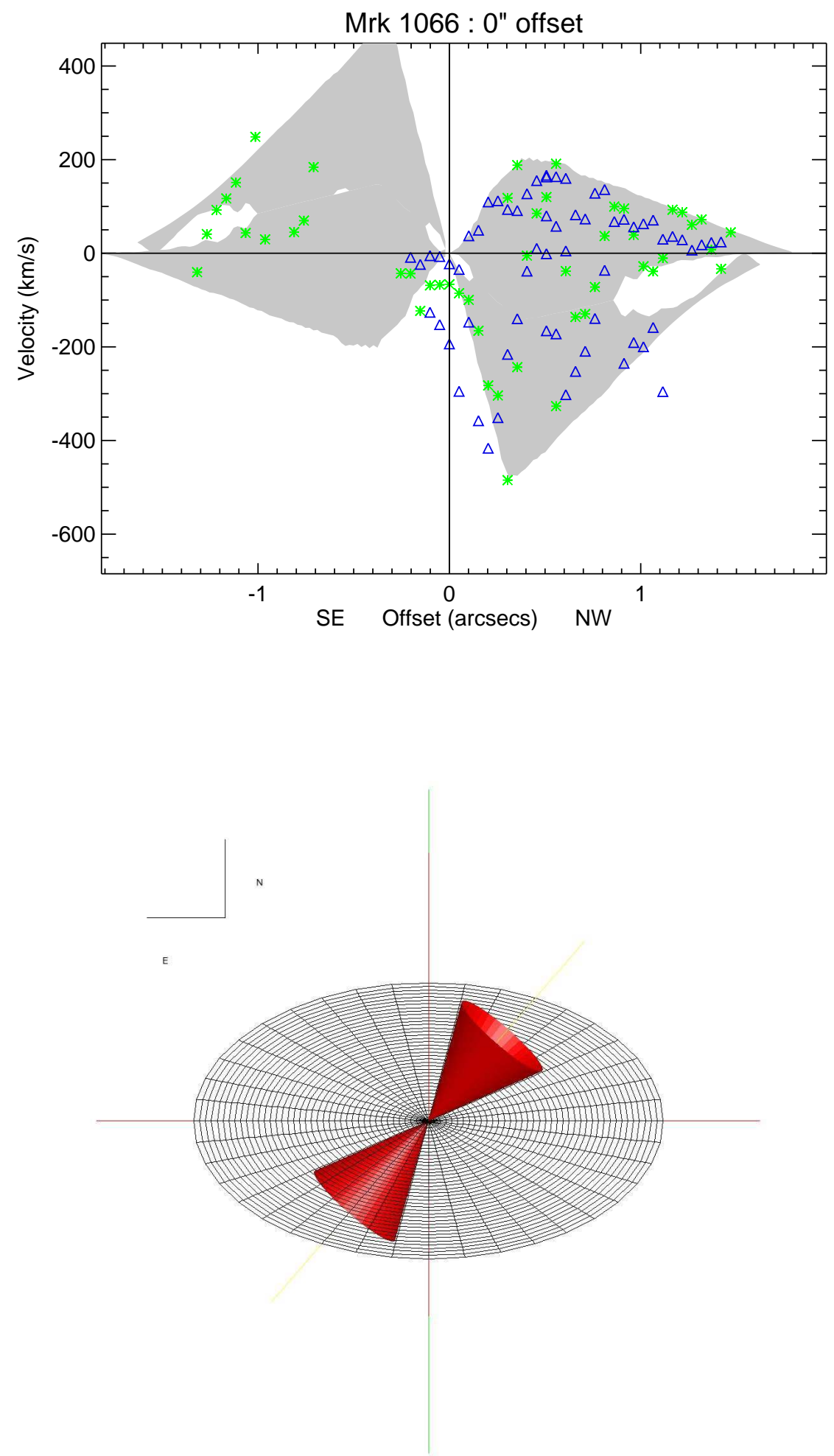

Figure 6.6 Above: Kinematic model of Mrk 1066. Below: Corresponding geometric model of the NLR with disk geometry. 


\subsubsection{NGC 1667}

NGC 1667 is a Seyfert 2 with a compact NLR spanning $\sim 1^{\prime \prime}$ in diameter in our STIS observation. NGC 1667 is unique in our sample in that we do not possess any images of its NLR. Continuum imaging shows a fairly unspectacular nucleus, with a bright central region overlapped by several dustlanes. The modeled kinematics provide a good fit to the data, although we cannot compare our modeled NLR geometry (bottom of Figure 6.7) with the available continuum filter imagin as it is difficult to distinguish where [O III] emission is present. As correspondance between imaging and our models is a parameter we use to create our most accurate fit, this discrepancy leaves room for improvement. Slit positions and corresponding spectral images of NGC 1667 STIS obserations are available in Figures A.5 and B.7.

\subsubsection{NGC 3227}

This is a highly reddened Seyfert 1 galaxy with an inclined disk that constrains NLR emission to the northeast of the nucleus. The modeled kinematics provide a fair fit to the data, with less successful fits in slit positions $\geq .5^{\prime \prime}$ from the nucleus. Modeling would not be possible using [OIII] spectra alone, as the single available G430L slit position does not return enough kinematic information to fit to a model, as shown in Figure D.6. Fortunately, supplementary G750M spectra map out a large portion of the NLR, which allow us to see prominent doppler-shifted $\mathrm{H} \alpha$ emission northeast of the nucleus. Though corresponding [O III] emission is lacking, we can assume that the observed emission is due to outflows in the NLR versus ionization and rotation within the host disk as the kinematics are asymmetric, contain blueshifted velocities $>400 \mathrm{~km} \mathrm{~s}^{-1}$, and contain double-peaked profiles in the narrow $\mathrm{H} \alpha$ emission line (see also Walsh et al. (2008)). The kinematics initially suggest that emission from both sides of a single cone are visible which would place the bicone axis near the plane of the sky. By constraining our model to fit the unified model, restricting the inclination from 

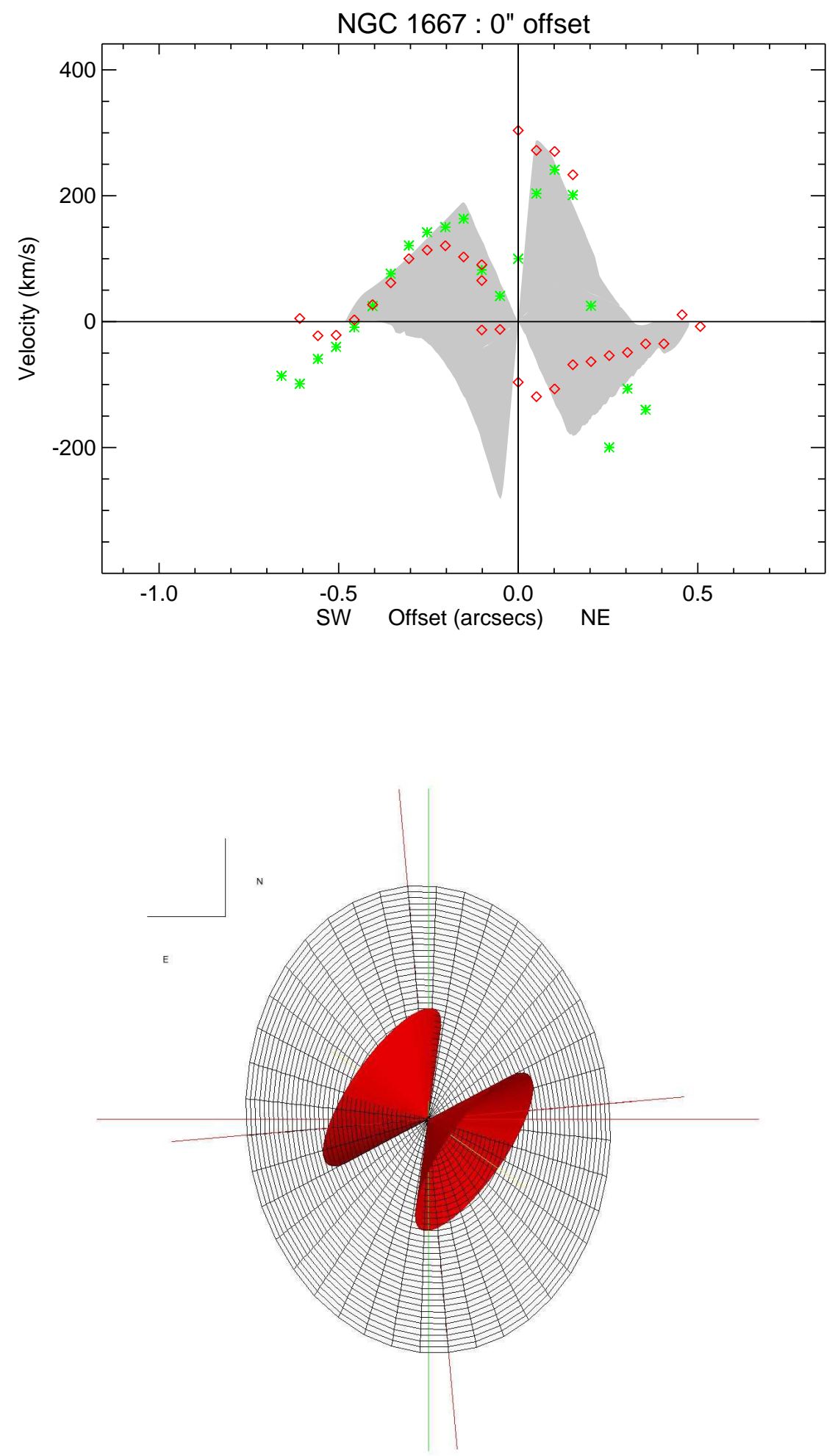

Figure 6.7 Above: Kinematic model of NGC 1667. Below: Corresponding geometric model of the NLR with disk geometry. 

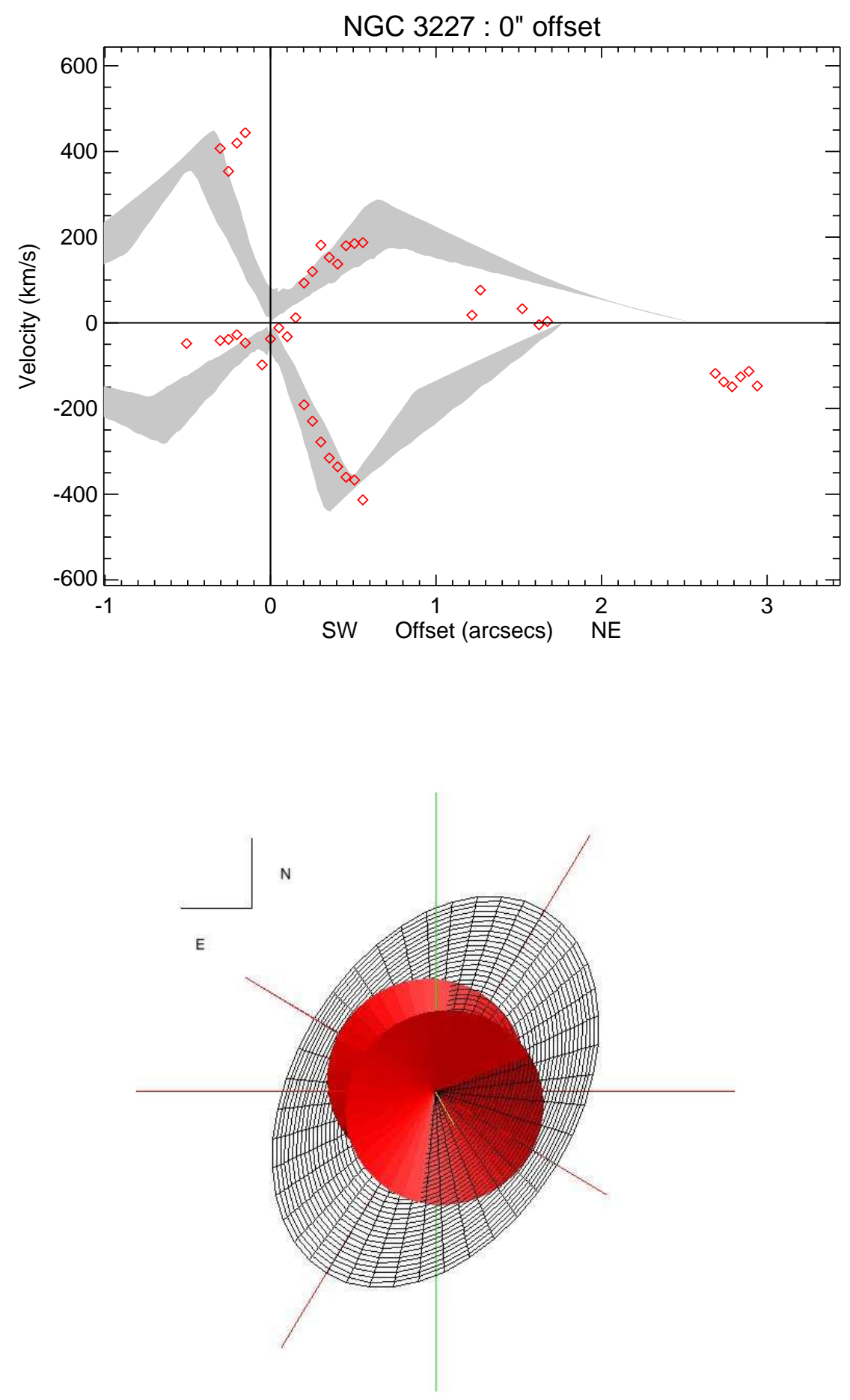

Figure 6.8 Above: Kinematic model for the central slit position of NGC 3227. Additional models for adjacent slits shown in Appendix D. Below: Corresponding geometric model of the NLR with disk geometry. 
obscuring the central engine, we find that the blue and redshifted kinematic components can be attributed to a single side of both cones and that the bicone axis is instead near our line of sight. This concurs with analysis in Crenshaw et al. (2002) which concludes that reddening is due to lukewarm absorbers coplanar with the host disk. Slit positions and corresponding spectral images of NGC 3227 STIS obserations are available in Figures A.6, B.8, and B.9.

\subsubsection{NGC 3783}

HST [OIII] imaging depicts Seyfert 1 galaxy NGC 3783 as an unresolved nuclear point source less than an arcsecond $(\sim 200 \mathrm{pc})$ in diameter. Emission detected with STIS reaches out to nearly twice that distance, with the $[\mathrm{OIII}] \lambda 5007$ line remaining visible at fluxes less than $1 \%$ of that at the continuum source. The resultant kinematics depict red and blue shifts on either side of the nucleus which, assuming a unified model, fits well with a biconical outflow axis nearly perpendicular to the plane of the sky such that the kinematics for each cone are entirely red/blue shifted. This pole-on geometry agrees fairly well the symmetric, compact point source in the [OIII] imaging and the Type 1 designation of the AGN.

This target was also observed by Müller-Sánchez et al. (2011), using the Keck OHSuppressing Infrared Integral Field Spectrograph (OSIRIS) and similar models based on the work in Crenshaw \& Kraemer (2000), where they publish a parameter set with a more edge-on inclination. Initially, the largest problem posed with this result is that imaging shows no elongated structure along the proposed bicone axis (P.A. $=-177^{\circ}$ ), where one should expect emission on either side of the nucleus corresponding to a single cone. Analysis of their technique shows that each spectrum that is observed is only fit with a single Gaussian, making it impossible to detect two separate components that are projected onto one another in the plane of the sky. This technique then at best can provide two kinematic components, one on each side of the nucleus, from which to fit a model. As they propose the outflow of the southern cone to be obstructed by the host disk, their model is only fit 

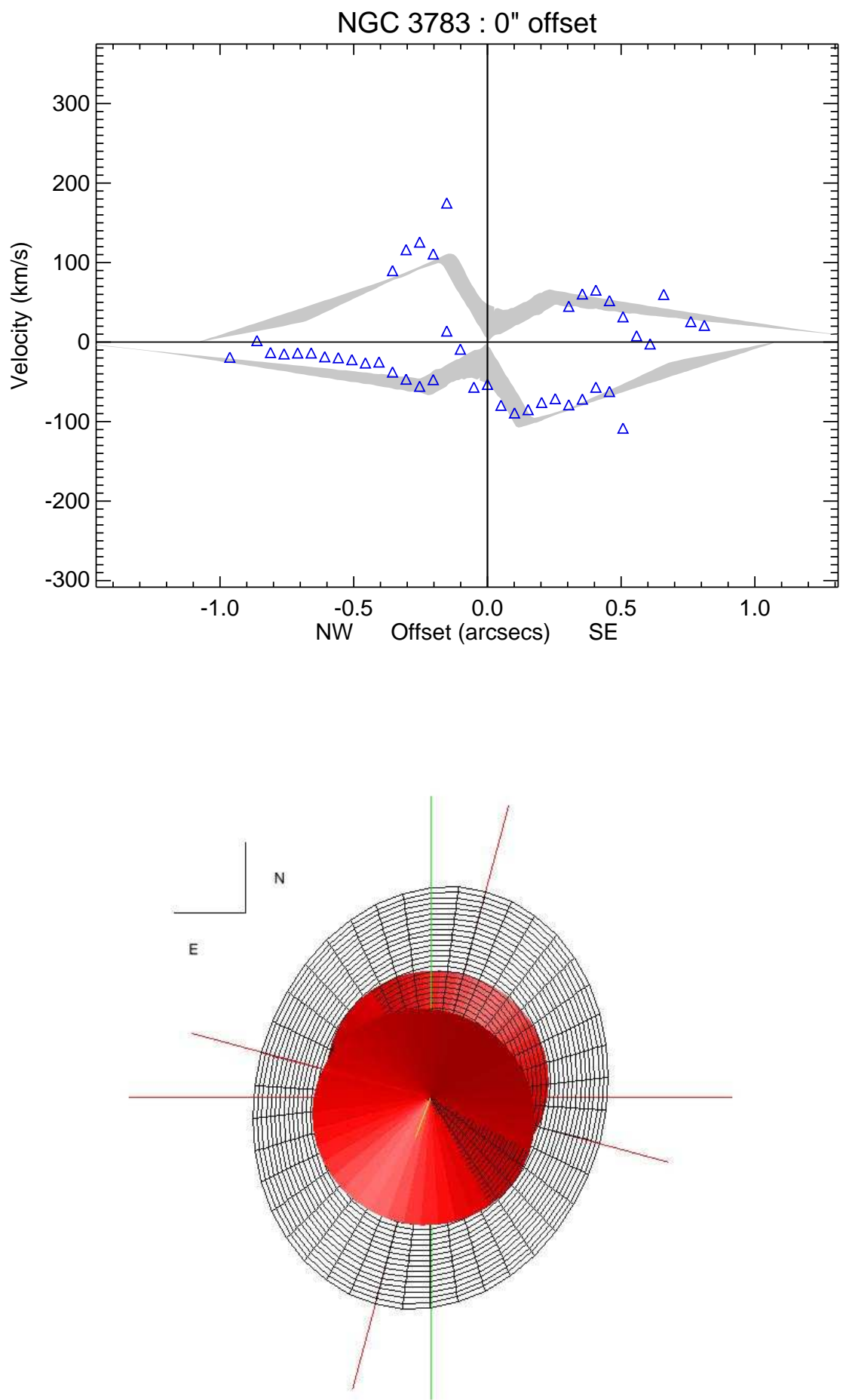

Figure 6.9 Above: Kinematic model of NGC 3783. Below: Corresponding geometric model of the NLR with disk geometry. 


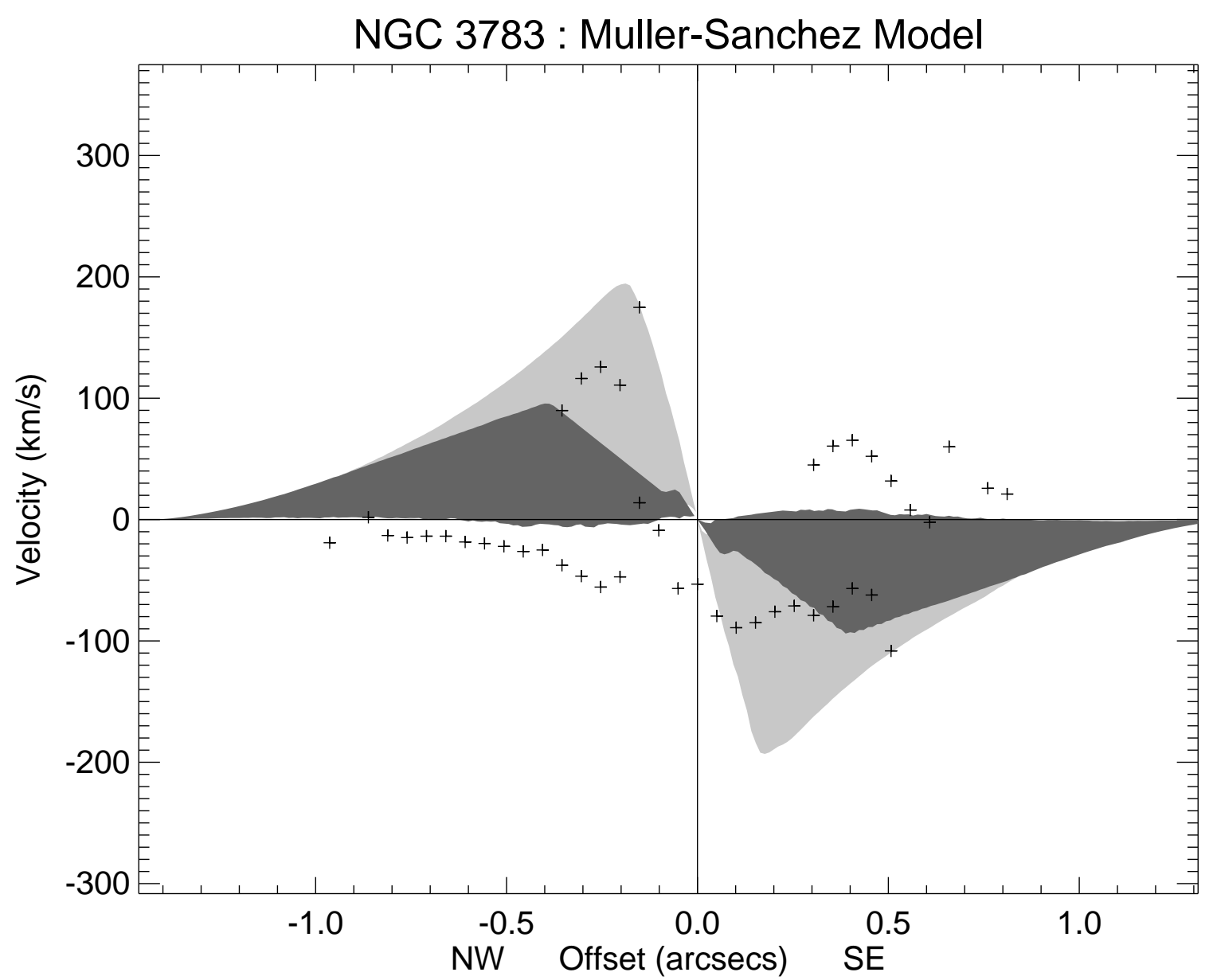

Figure 6.10 Radial velocities of [O III] measurements for the central slit position of NGC 3783 with overlayed model using parameters from Müller-Sánchez et al. (2011).

to a single kinematic component of the biconical outflow. As mentioned in Section 3.4, it should be impossible to determine essential parameters, including inclination and opening angle, without at least two adjacent kinematic components. Additionally, they do not employ their geometrical model as a parameter as we do. Though their kinematic model for NGC 3783 clearly states that the southern cone is completely obstructed, they provide a geometric model where the NLR outflow is bisected by the host disk, similar to our own model for NGC 3227. Slit positions and corresponding spectral images of NGC 3227 STIS obserations are available in Figures A.6, B.8, and B.9. 
Fitting their model to our kinematics, shown in Figure 6.10, shows that half of the kinematics are not accounted for using their parameters and by comparing the kinematics with the spectral image in Figure B.10 we see that the kinematics avoided by their model are the higher flux lines, suggesting they do not account for a significant portion of the NLR. More importantly, their model indicates a view far outside of the bicone, which is not consistent with the unified model view of a Seyfert 1.

\subsubsection{NGC 4051}

Observations of NGC 4051 are of particular interest as it is a NLS1 (see Section 6.1.2). The modeled kinematics provide a fair qualitative fit to the data, matching well to the inner accelerating blueshifted outflows. Using our final model parameters, we find that the axis of the bicone is inclined $12^{\circ}$ away from pole-on, with our line of sight running near the edge of the NLR, between the inner and outer opening angles of the outflow. As we assume the highly blueshifted radial velocities are due to a biconical outflow, there is a noticeable lack of corresponding highly redshifted velocities near the nucleus. Combining the outer

opening angle of the kinematic model with the inner disk geometry (Barbosa et al. 2009), suggests that the lack of redshifted outflow is likely due to disk obscuration. Slit positions and corresponding spectral images of NGC 4051 STIS observations are available in Figures A.7, B.10 and B.11.

While we do find that the inclination of NGC 4051 is near pole-on at $12^{\circ}$, we also find that the angle between the outer edge and our line of sight to be nearly identical. Analysis done for the three additional availabe NLS1s, Akn 564, Mrk 766 and Mrk 1040, show similar highly blueshifted velocities near their nuclei, suggesting that a near pole-on orientation may be common. Unfortunately, the emission for these targets is too compact to fit with an accurate outflow model. Further study on the asymmetrical distribution of outflow velocities on either side of nuclei for both NLS1s and BLS1s will be required to test the pole-on hypothesis for 

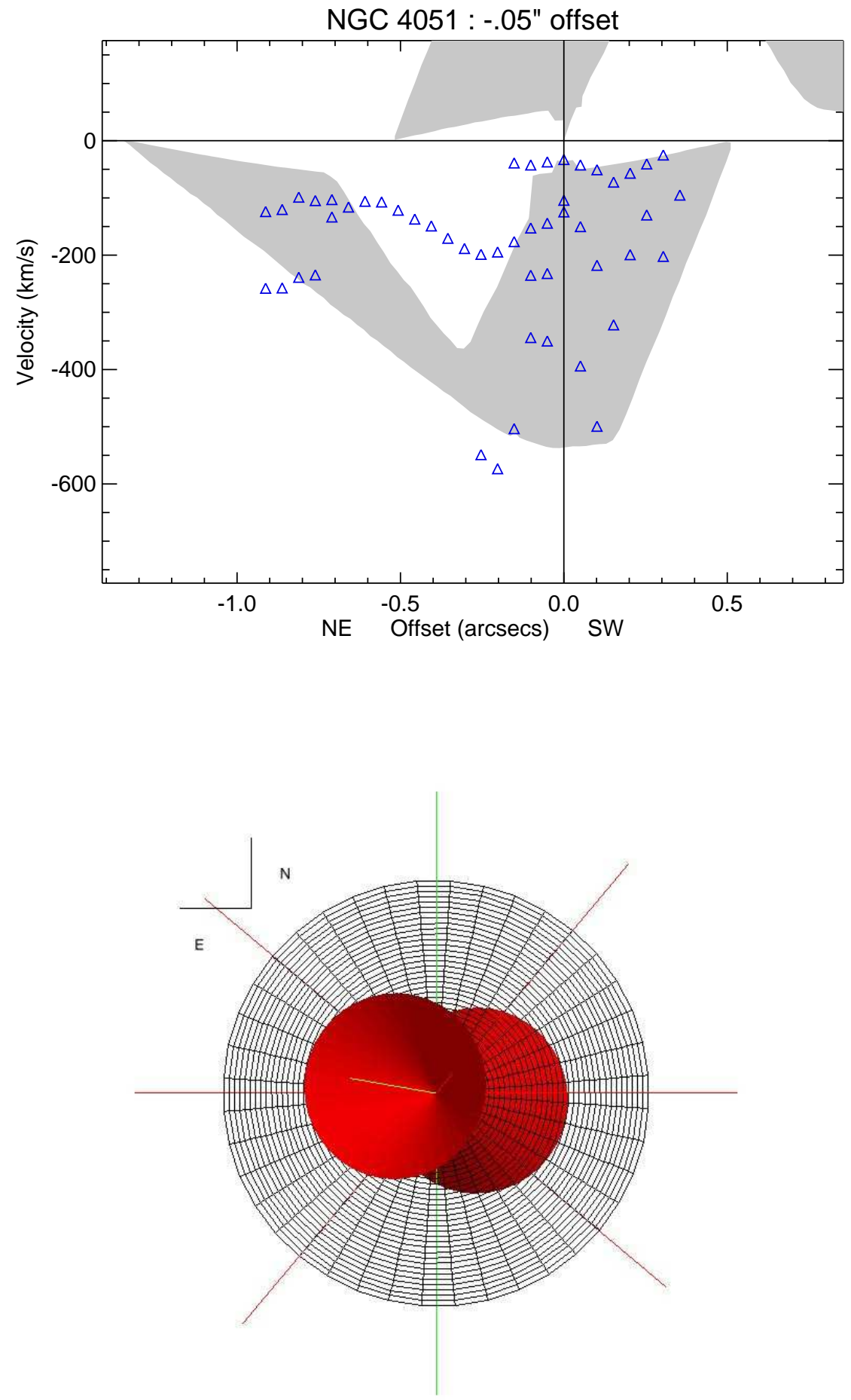

Figure 6.11 Above: Kinematic model for the central slit position of NGC 4051. Additional models for adjacent slits shown in Appendix D. Below: Corresponding geometric model of the NLR with disk geometry. 
NLS1s.

\subsubsection{NGC 4507}

This is a Seyfert 2 galaxy that features two extended knots of emission. The first, $0.5^{\prime \prime}$ northwest of the nucleus along the STIS slit, is traveling near systemic velocity and fits well in the final kinematic model. The second knot, at $1^{\prime \prime}$ northwest of the nucleus, is highly blueshifted at $1000 \mathrm{~km} \mathrm{~s}^{-1}$. This second knot is abnormal in that it is a spatially resolved, high velocity knot at a large distance from the nucleus such that it cannot be fit with our model. Rogue high velocity clouds such as this have also been documented in the kinematics of NGC 4151 (Das et al. 2005), though they are located at radii near the peak of the velocity curve versus the end of the curve in this case. Two other kinematic components exist in the blueshifted quadrant to the northwest of the nucleus, thus it is unlikely to be a part of the typical NLR outflow and is possibly a cloud near the axis of the bicone. Though many highly blueshifted velocities exist near the nucleus, the blueshifted component was fit such that the higher flux velocities (Figure C.26) took priority. Additionally, opening the bicone to accommodate the high, southeast velocities would allow for the viewing of the central engine, which does not currently occur as our line of sight runs in between the inner and outer opening angles of the NLR. Slit positions and corresponding spectral images of NGC 4507 STIS obserations are available in Figures A.7 and B.11.

\subsubsection{NGC 5506}

This is a Seyfert 2 AGN that resides in a near edge-on host. From imaging (Figure A.9), a single, well-defined NLR cone axis appears to be near the plane of the sky and perpendicular to the host disk. Spectra available for this target consist solely of the slitless Ruiz et al. (2005) observations which can be fit well with a model that also agrees with the imaging. Though we were successful in modeling the NLR, the neutral column density $\left(\mathrm{N}_{H}\right)$ for NGC 

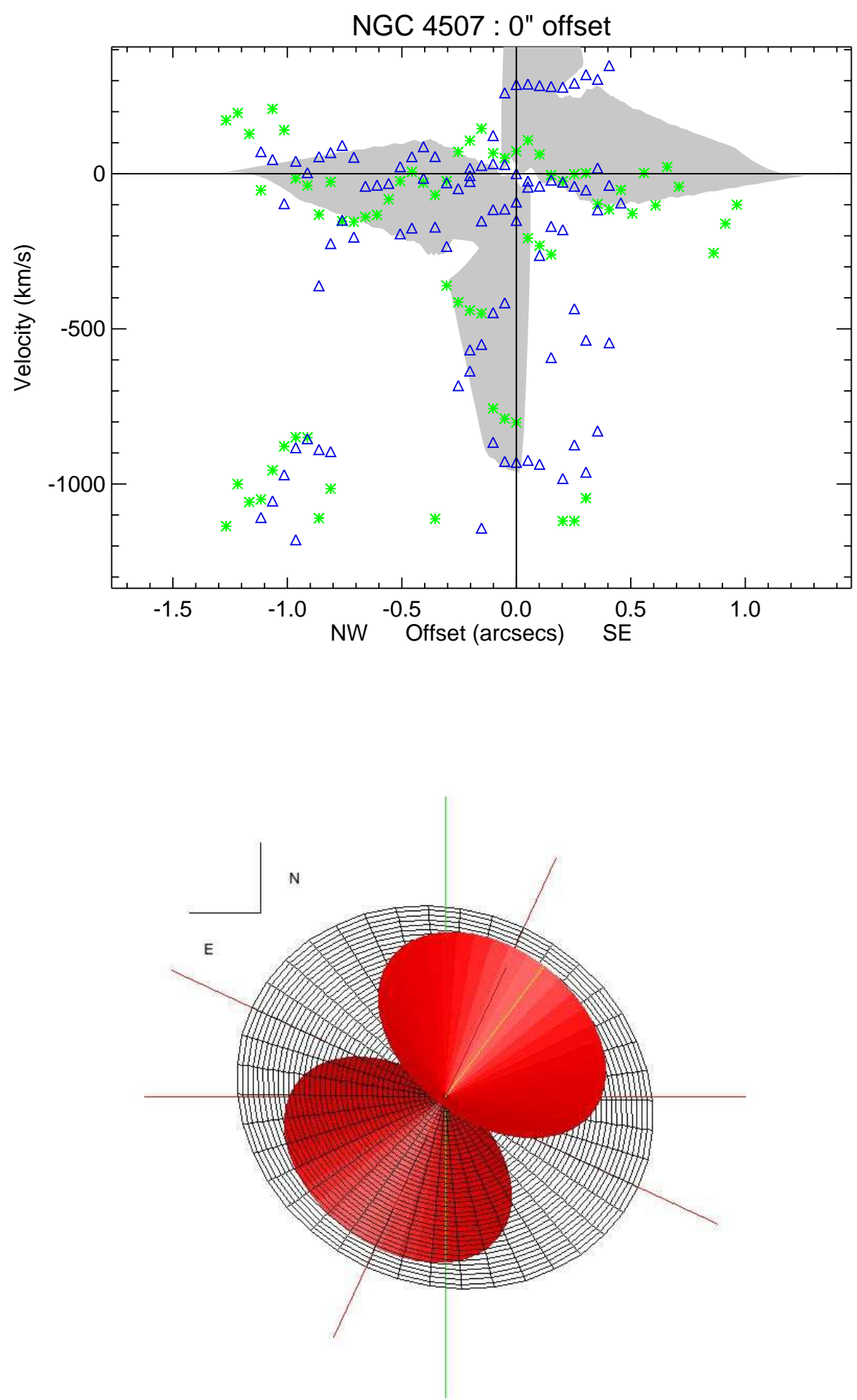

Figure 6.12 Above: Kinematic model of NGC 4507. Below: Corresponding geometric model of the NLR with disk geometry. 

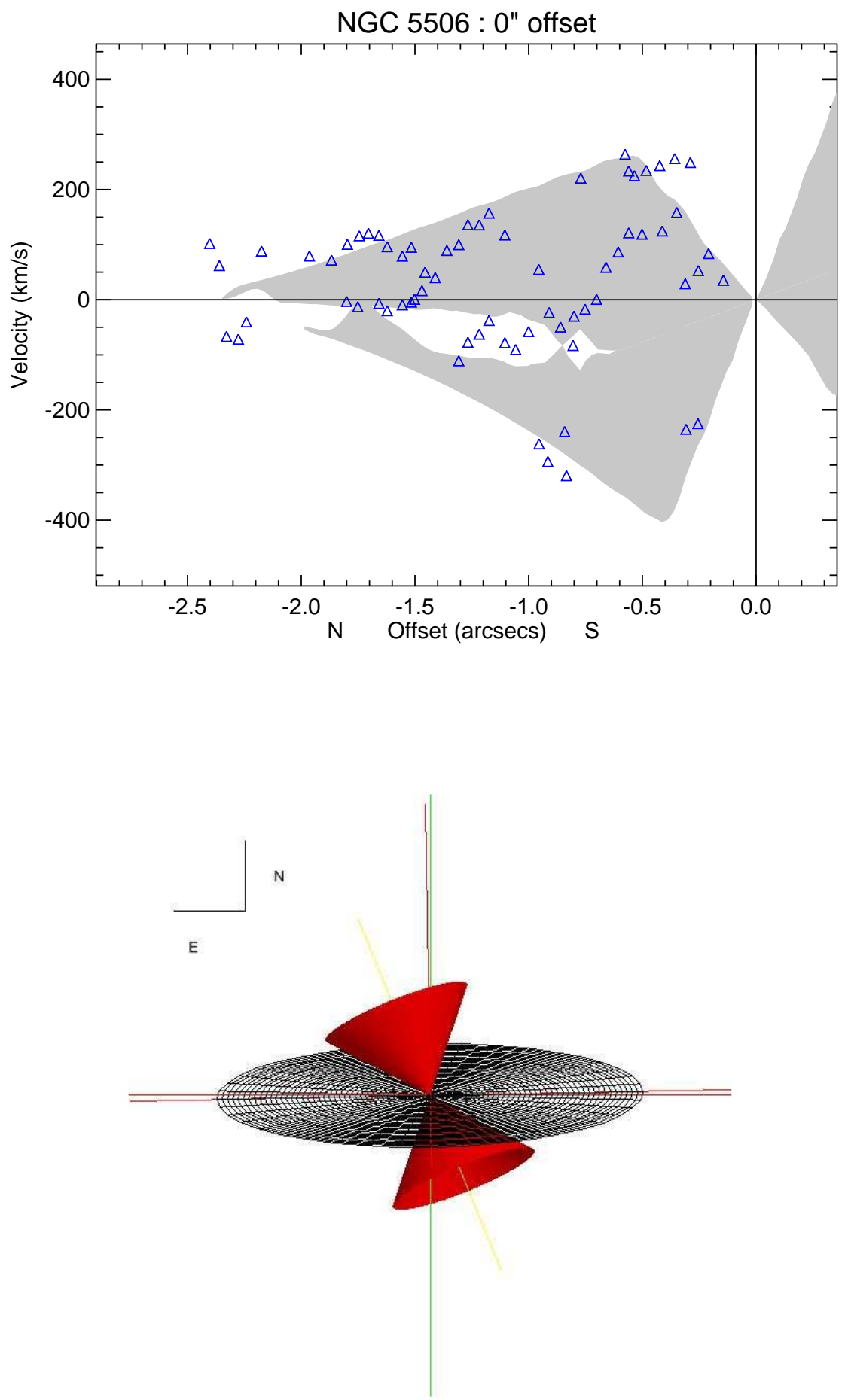

Figure 6.13 Above: Kinematic model of NGC 5506. Below: Corresponding geometric model of the NLR with disk geometry. 
5506 is near $3 \times 10^{22} \mathrm{~cm}^{-2}$ (Trippe et al. 2010; Shu et al. 2007) which, as explained in Section 7.1, is exceedingly low for an AGN we would be observing near edge-on. As this is the only edge-on Seyfert 2 in our modeled sample, it is difficult to determine if the AGN itself is truly a Type 2 AGN or if the host disk is extinguishing BLR emission and we are seeing a NLR carved by the disk. Further research in the infrared, detailed in Section 8.2.1, would help clarify whether or not extinction is affecting the perceived NLR geometry.

\subsubsection{NGC 5643}

This is a Seyfert 2 which has a well-defined triangular emission region east of the nucleus, as seen in Figure A.9. Filamentary structure within this ionized region appear to follow spiral arms in the host disk (Morris et al. 1985). While G430L and G750M long-slit observations are available, their position angle of $-128^{\circ}$ places them outside the NLR and only detects nuclear emission. Kinematics from slitless observations show that the majority of the extended emission is redshifted, with blueshifted velocities only visible near the nucleus. Two $1^{\prime \prime}$ wide pseudo-slit positions of $110^{\circ}$ and $125^{\circ}$ (Figures D.11 and D.12) were used to cover the majority of the extended emission. We fit a model to these kinematics that corresponds to a single, wide opening-angle cone inclined $65^{\circ}$ from our line of sight. Similar to Mrk 573, the extended [O III] emission seen in the imaging appears to result from the intersection between the host disk and the ionization bicone. While the geometric model suggests that extended blueshifted emission should be observed west of the nucleus, the emission may be quenched by perhaps a warped disk or the presence of a dust lane to the southwest of the nucleus where traces of emission $4.5^{\prime \prime}$ and $8.2^{\prime \prime}$ west of the nucleus could represent emission seen through less obscured portions of the disk (Simpson et al. 1997). Spectroscopic observations by Schmitt et al. (1994) support these postulations as line emission to the west of the nucleus is more heavily reddened than that to the east. 

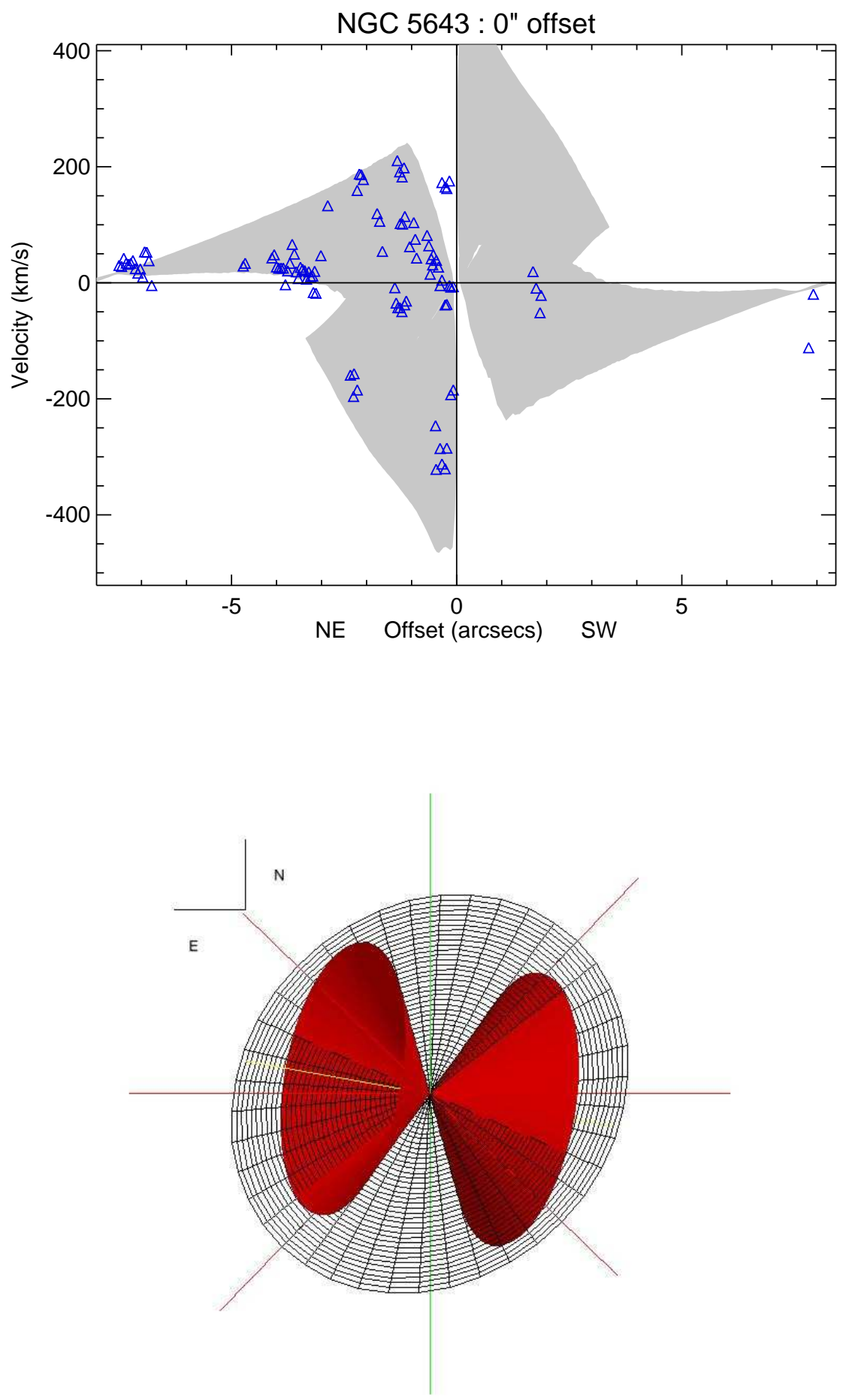

Figure 6.14 Above: Kinematic model of NGC 5643. Below: Corresponding geometric model of the NLR with disk geometry. 


\subsubsection{NGC 7674}

GNC 7674 is a Seyfert 2 with a distribution of radial velocities similar to Circinus with asymmetric extended emission to the southeast with high blueshifts and near systemic redshifts. The modeled kinematics provide an excellent fit to the data, clearly fitting the two observed components and what remains of the other velocities. Our model suggests the extended emission is due to a single cone, with the other cone obstructed by the host disk. Slit positions and corresponding spectral images of NGC 4507 STIS obserations are available in Figures A.11 and B.15.

\subsection{Distinct Unmodeled Targets}

\subsubsection{IRAS 11058-1131}

This is a Seyfert 2 that shows a likely intersection between the NLR and host disk in the visible S-shaped NLR seen in Figure A.1. The kinematics of this target have been deemed 'complex' as no individual kinematic components can be seen in the radial velocity data. Velocities shown in Figure C.5 appear to be roughly rotational, as velocities further from the nucleus do not decelerate back toward systemic, but end with blueshifted values to the northeast and redshifted values to the southwest, reaching maximum velocities of $\sim 200 \mathrm{~km}$

$\mathrm{s}^{-1}$. This implies a counter-clockwise rotation, which agrees with the apparent winding-up of the spiral arms that are illuminated. Velocity gradients responsible for the knotty, 'complex' kinematics observed may be due to in situ acceleration similar to Mrk 573, though these gradients fail to align with specific flux peaks in the data.

\subsubsection{Mrk 509}

This is a Seyfert 1 that likely contains a tidal tail in its central regions. Seen in both optical continuum and [O III] imaging (Figure A.3), this feature is composed of several knots $2.2^{\prime \prime}$ 

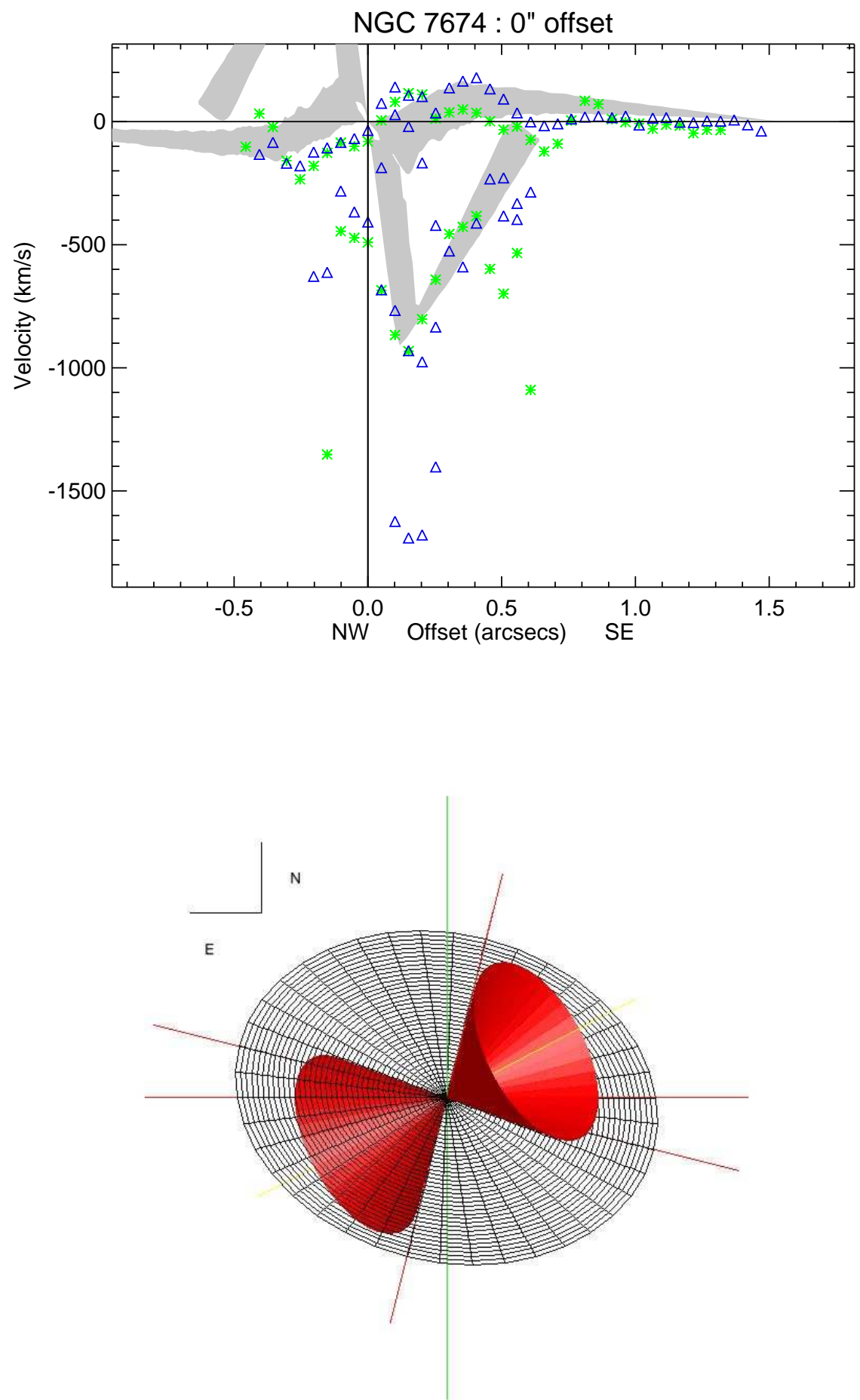

Figure 6.15 Above: Kinematic model of NGC 7674. Below: Corresponding geometric model of the NLR with disk geometry. 


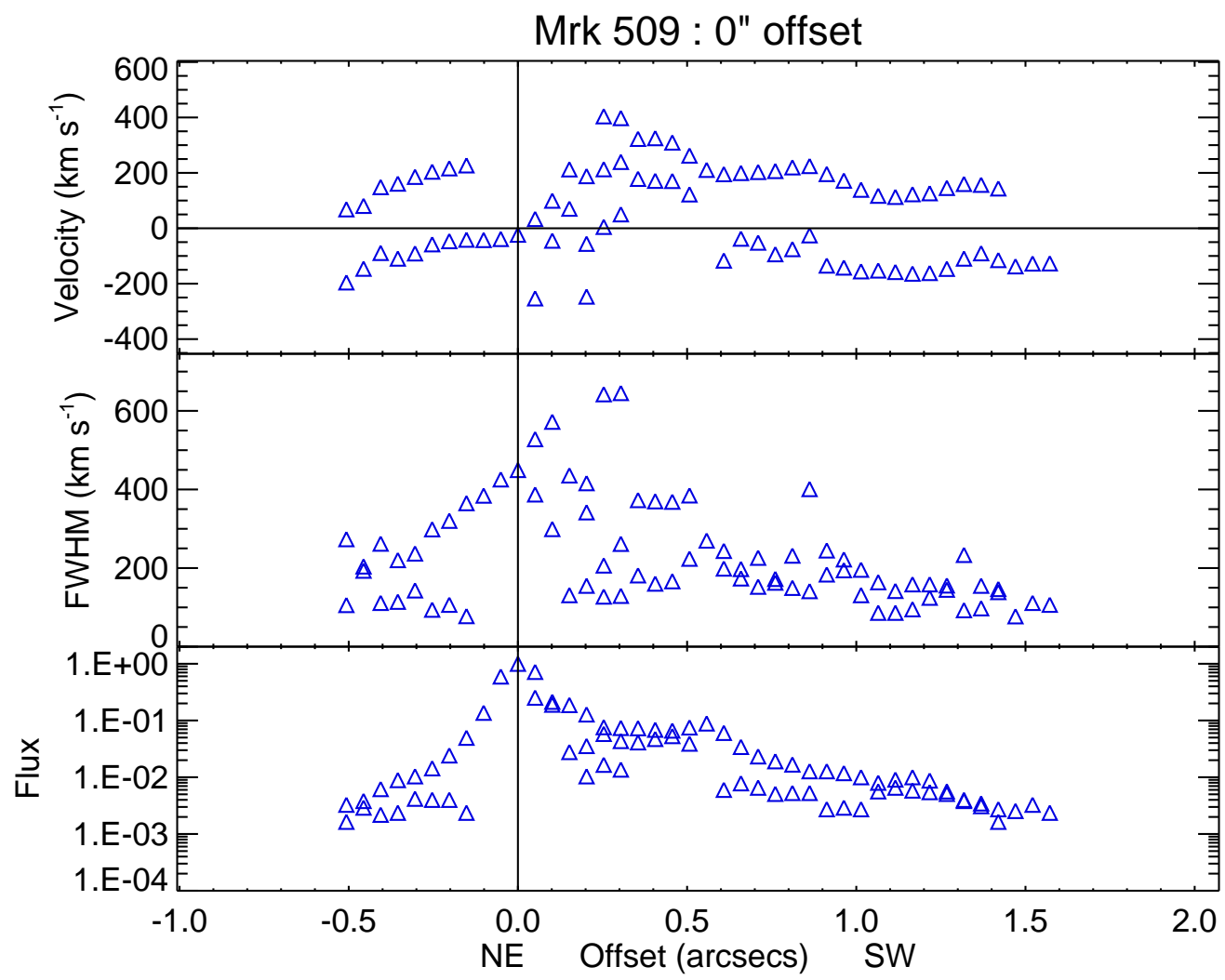

Figure 6.16 [O III] radial velocities, FWHM, and normalized fluxes of Mrk 509. Kinematics for the observed tidal tail reside in the inner $.5^{\prime \prime}$ southwest of the nucleus.

in length at a position angle of $-13^{\circ}$ before jutting towards the nucleus from the southwest at a position angle of $-110^{\circ}$. As the $H S T$ STIS slit was positioned favorably at $75^{\circ}$, we are able to observe the inner portion of the tail and analyze its kinematics. Kinematics to the southwest of the nucleus show redshifted velocities peaking near $400 \mathrm{~km} \mathrm{~s}^{-1}$, which correspond to the highest flux lines at those positions. Coupling this data with the apparent projection of the tidal tail above the host galaxy disk suggests that the bright, inner portion of the tidal tail is inflowing. Should this be the case, we may be viewing a minor merger with a dwarf galaxy, and this system would provide a great opportunity to study the fueling of an AGN by a minor merger in progress. Assuming that the redshifted kinematic component southwest of the nucleus is an inflowing tidal tail, the remaining velocities do not provide 


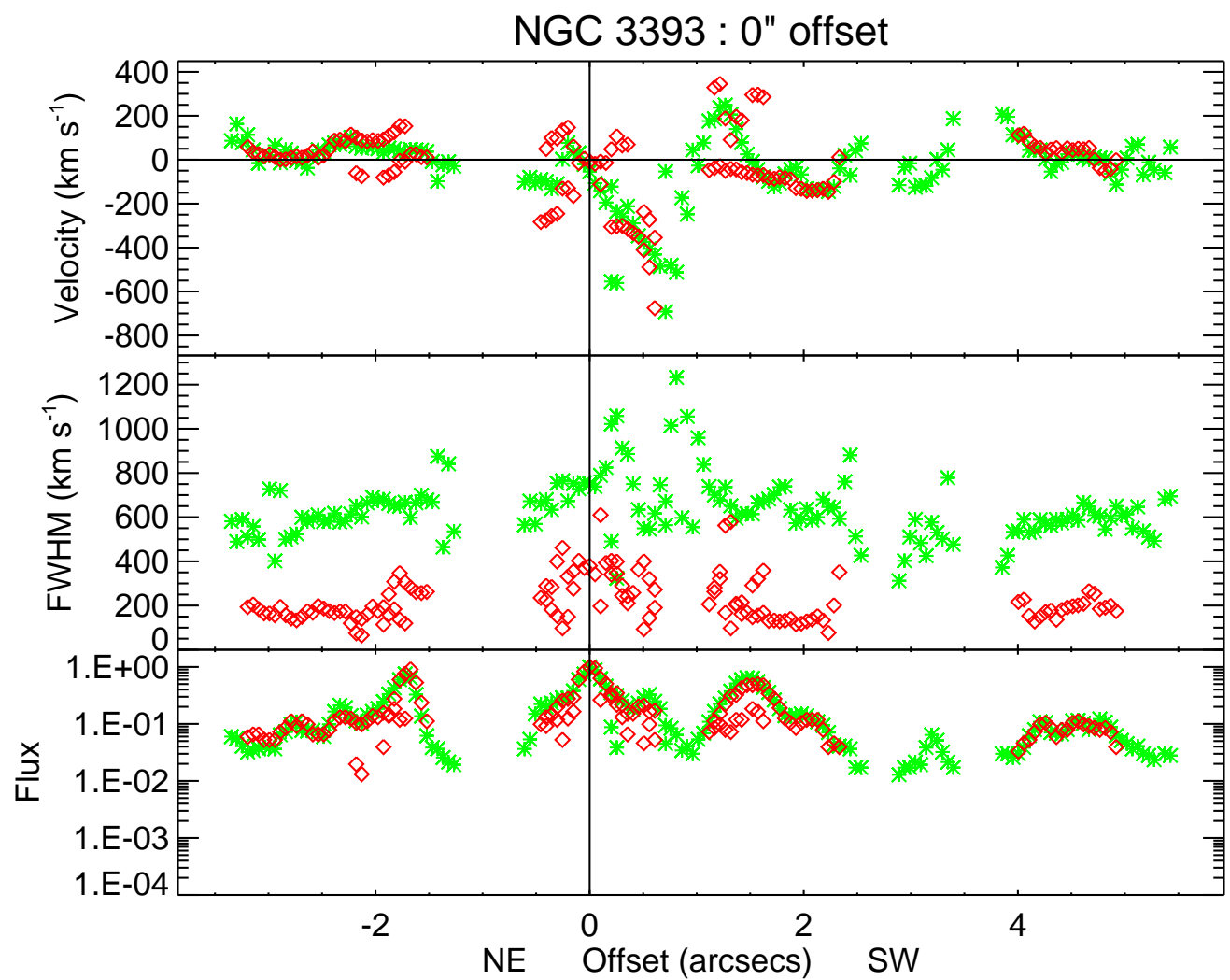

Figure 6.17 Radial velocities, FWHM, and normalized fluxes of NGC 3393. Green stars depict [O III] lines measured using the G430L grating, red diamonds depict $\mathrm{H} \alpha$ lines measured using the G750M grating.

enough information to fit a model to the true outflowing kinematics. Further observations of the entire tail, particularly the extended linear feature, would clarify which direction the feature is moving and whether it is inflowing.

\subsubsection{NGC 3393}

NGC 3393 is another Seyfert 2 that contains an S-shaped NLR, shown in Figure A.6 which shows turbulent kinematics across several knots of emission, similar to Mrk 573 and IRAS 11058-1131. Though the NLR is well resolved (distinct knots visible over several arcseconds), it is unclear whether any distinct kinematic components are present. Two sets of high velocities exist to the southwest of the nucleus, one blueshifted component peaking at . $75^{\prime \prime}$ 
and one redshifted component peaking at 1.25", which for a Seyfert 2 AGN would likely be two components for one cone to the southwest, inclined close to the plane of the sky. We believe this is unlikely as imaging shows symmetric lobes of emission on either side of the nucleus which would not be replicated in the kinematic data. An alternative method to fitting the kinematics is to move the central position of the nucleus southwest $\sim .9^{\prime \prime}$. This would imply that the location of the continuum peak in the spectral image would not suffice as the true position of the AGN, possibly due to obscuration and reflection of the central engine. Peaks in flux to either side of the new center support the possibility of obscuration over the AGN. At this new position, the two previous high velocity peaks are now on each side of the center, combined with the extended, near-systemic velocities further out to provide four kinematic components to model. Figure 6.18 shows a model fit to the altered velocity dataset.

Though the model fits NLR velocities well, there is not enough evidence to justify the altered placement of the kinematic center. Additionally, the difficult nature of fitting the observed NLR kinematics with a model of a single outflowing AGN may be due to possible dual AGN residing within NGC 3393. Separated by $\sim 150$ pc ( .6" $)$, Fabbiano et al. (2011) report the central knot of emission in imaging to house two active black holes using Chandra observations in the 6-7 keV band. They discount either centroid as an interaction between a single AGN and its environment, as the northeast source has a far-IR luminosity comparible to that of the entire estimated intrinsic nuclear emission, thus too bright to be reflected emission, and the southwest source contains no $\mathrm{H} \alpha$ and [O III] emission which would be required in a reflection scenario. As Fe-K $\alpha$ emission is seen from the southwest source, obscuration cannot be used to explain the missing optical emission. 


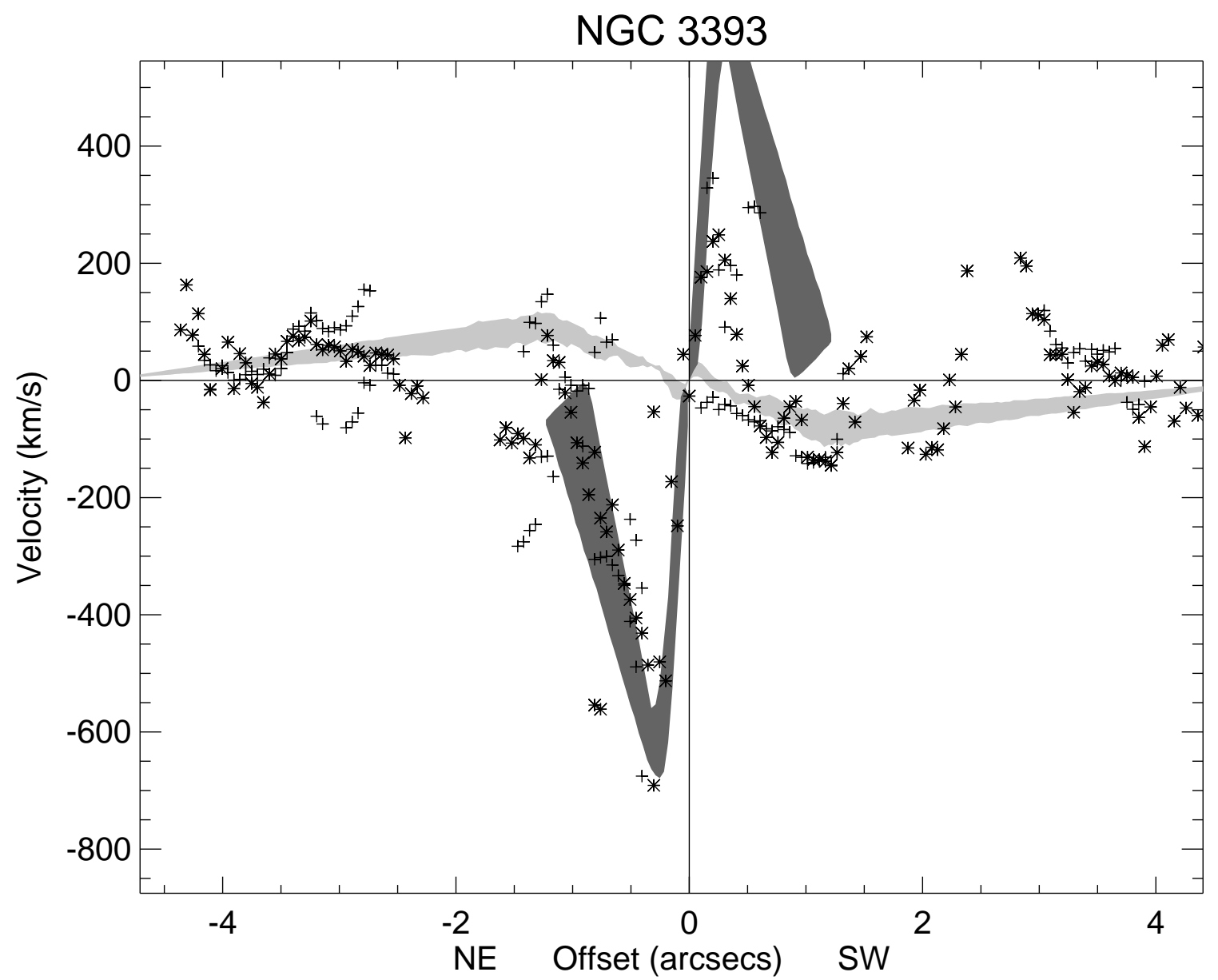

Figure 6.18 Radial velocities measurements of [O III] using G430M (*) and G430L (+) gratings for the central slit position of NGC 3393 with overlayed model assuming a shifted central location of $1^{\prime \prime}$ southwest.

\subsubsection{NGC 3516}

An atypical Seyfert 1 AGN as it contains an S-shaped NLR (Figure A.6) more often seen in Type 2 AGN, NGC 3516 is our prototypical ambiguous outflow system. Similar to Mrk 3 and Mrk 573, this S-shape is likely due to dust lanes of the host disk becoming ionized as they intercept the NLR bicone. Figure 6.19 shows a well-defined symmetric kinematic component on either side of the nucleus traveling in opposite directions which correspond to this extended emission seen in imaging. While the kinematics seen in these components match signatures of outflow employed by our model, we cannot fit the data as the components are not adjacent to 


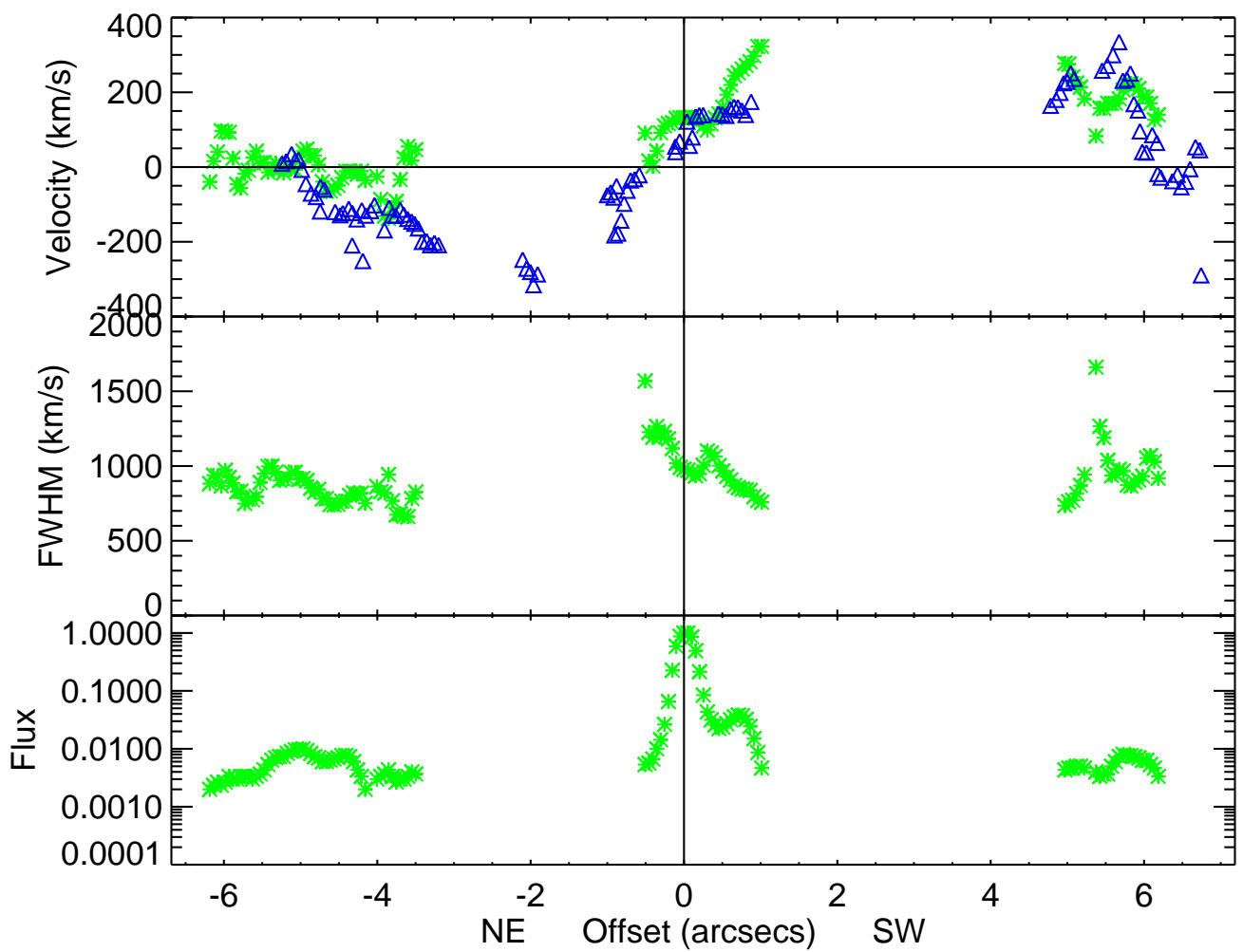

Figure 6.19 [O III] radial velocities, FWHM, and normalized fluxes of NGC 3393. Green stars designate G430L long-slit data, blue triangles designates a $1^{\prime \prime}$ width psuedo-slit observation from G430M slitless data (Ruiz et al. 2005) along the same position angle.

each other (see Section 3.4). It is not clear why components that correspond to the opposite side of each cone are present, though it may be possible that they reside near the nucleus and are outshone by its flux or that there is a lack of an ionizing medium to detect where the components exist.

Though we cannot fit a kinematic model to NGC 3516, we attempted to recreate the NLR geometrically using the following assumptions:

1.) The side of each cone nearer the plane of the sky must intercept the host disk (using a Kinney et al. (2000) disk geometry) such that the resultant intersection geometry matches that seen in the [O III] imaging. 
2.) The opening angle must be wide enough such that we are viewing the central engine (i.e. the geometric model must be a Type 1 AGN).

Using these parameters, we were able to construct a geometric model that agreed with the [O III] imaging using a bicone position angle, inclination, and outer opening angle of $40^{\circ}, 50^{\circ}$, and $50^{\circ}$ respectively. Unfortunately, the resultant kinematic model did not agree with the observed radial velocities and attempts to reconcile the two models have been unsuccessful. 


\section{CHAPTER 7}

\section{EXPANDED SAMPLE RESULTS}

After analyzing the entirety of the remaining targets using the same techniques as with Mrk 78 and Mrk 573, we have found that 12 additional AGN show distinct kinematics characteristic of biconical outflows seen in our previously modeled targets. Excluding NGC 5506 until further clarification can be made on how the host disk affects its NLR, our AGN sample includes 16 targets in total. In this section, we compare our modeled AGN parameters with several observed AGN parameters likely to be affected by viewing angle, listed in Table 7.1, and determine how NLR geometry might be correlated with observable parameters of AGNs.

Bolometric luminosities, integrated across each AGNs spectral energy distribution (SED), were obtained from the literature (Woo \& Urry 2002). SMBH masses of all Seyfert 1 were determined using reverberation mapping, where BLR size, estimated from the time lag between corresponding ionizing continuum and broad line emission fluxes, and broad-line velocities are combined to calculate the AGN black hole mass. Seyfert 2 SMBH masses were calculated using either maser kinematics, observed in NGC 1068 and Circinus, to measure the spatially resolved dynamics near the $\mathrm{SMBH}$, or stellar velocity dispersions in the remaining Seyfert 2 AGN to indirectly constrain SMBH mass as they correlate well with black holes masses determined from spatially resolved kinematics (Tremaine et al. 2002).

In Figures 7.1 and 7.2 we show the distribution of AGN polar angles (the inclination from our LOS, or the angle between the bicone axis and our LOS) and opening angles respectively of our total modeled sample. Note that while our sample size is small, it also covers a full range in both inclination and opening angle. The distribution of polar angles shows a trend of Seyfert 1s having lesser inclinations from our line of sight and Seyfert 2s being more inclined. It should be noted that a requisite for our kinematic model was that it agree with the unified model. As each of our models is also constrained by available [O III] 
Table 7.1 Physical properties of modeled AGN sample. H $\beta$ FWHM values gathered for Seyfert 1s only.

References: (1) Woo \& Urry (2002) and references therein, (2) Peterson et al. (2004), (3) Bentz et al. (2006), (4) Denney et al. (2009), (5) Denney et al. (2010), (6) Meléndez et al. (2008), (7) Greenhill et al. (2003), (8) Heckman et al. (2005), (9) Bian \& Gu (2007), (10) Gu et al. (2006), (11) Alonso-Herrero et al. (2011), (12) Shu et al. (2007) and references therein, (13) Crenshaw \& Kraemer (2012), (14) Markowitz et al. (2009), (15) Vestergaard \& Peterson (2006), (16) Grupe et al. (2004), (17) This work

\begin{tabular}{|c|c|c|c|c|c|c|c|c|c|c|}
\hline Target & Type & 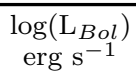 & Ref. & $\begin{array}{c}\log (\mathrm{M}) \\
\mathrm{M}_{\odot}\end{array}$ & Ref. & $\mathrm{L} / \mathrm{L}_{e d d}$ & $\begin{array}{c}\mathrm{N}_{H} \\
\times 10^{22} \mathrm{~cm}^{-2}\end{array}$ & Ref. & $\begin{array}{c}\mathrm{H} \beta \mathrm{FWHM} \\
\mathrm{km} \mathrm{s}^{-1}\end{array}$ & Ref. \\
\hline Circinus & 2 & 42.08 & 6 & 6.23 & 7 & 0.0056 & 430 & 12 & - & 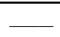 \\
\hline Mrk 3 & 2 & 44.54 & 1 & 8.65 & 1 & 0.0061 & 136 & 12 & - & - \\
\hline Mrk 34 & 2 & 46.13 & 8 & - & - & - & $>100$ & 12 & - & - \\
\hline Mrk 78 & 2 & 44.59 & 1 & 7.87 & 1 & 0.041 & 57.5 & 12 & - & \\
\hline Mrk 279 & 1 & 45.04 & 8 & 7.54 & 2 & 0.25 & $>0.034$ & 13 & 5411 & 15 \\
\hline Mrk 573 & 2 & 44.44 & 1 & 7.28 & 1 & 0.11 & $>100$ & 12 & - & 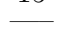 \\
\hline Mrk 1066 & 2 & 44.55 & 1 & 7.01 & 1 & 0.27 & $>100$ & 12 & - & - \\
\hline NGC 1068 & 2 & 44.98 & 1 & 7.23 & 1 & 0.44 & $>1000$ & 12 & - & - \\
\hline NGC 1667 & 2 & 44.69 & 1 & 7.88 & 1 & 0.051 & $>100$ & 12 & - & ـ \\
\hline NGC 3227 & 1 & 43.86 & 1 & 6.88 & 5 & 0.013 & 0.35 & 14 & 3823 & 17 \\
\hline NGC 3783 & 1 & 44.59 & 1 & 7.47 & 2 & 0.047 & 0.57 & 13 & 2612 & 17 \\
\hline NGC 4051 & 1 & 43.56 & 1 & 6.24 & 4 & 0.17 & 4.11 & 13 & 1170 & 16 \\
\hline NGC 4151 & 1 & 43.73 & 1 & 7.66 & 3 & 0.031 & 5.32 & 13 & 6421 & 15 \\
\hline NGC 4507 & 2 & 42.92 & 6 & 6.13 & 10 & 0.048 & 43.9 & 12 & - & \\
\hline NGC 5506 & 2 & 44.05 & 6 & 6.88 & 10 & 0.11 & 2.8 & 12 & - & - \\
\hline NGC 5643 & 2 & 43.98 & 6 & 6.79 & 10 & 0.12 & 70.7 & 12 & - & - \\
\hline NGC 7674 & 2 & 45.00 & 11 & 7.58 & 9 & 0.27 & $>1000$ & 12 & - & 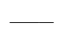 \\
\hline
\end{tabular}

imaging, we are able to remove the ambiguity of models that could be fit with two individual parameter sets that are inclined perpendicular to one another (as discussed in Section C.34). Thus, we believe this distribution to be significant and unbiased by our initial unified model requirement. The second distribution of opening angles show Seyfert 1s and Seyfert 2s have no preference for specific angles. This broad range of opening angles points to the differences in the inner torus-like structure geometry surrounding each AGN.

\subsection{Column Density}

The unified model assumes the presence of a torus-like structure surrounding the AGN that obstructs our view to the central engine in Type 2 targets. Thus, a simple prediction is that as we observe an AGN further from the axis of its NLR and closer to the toroidal 


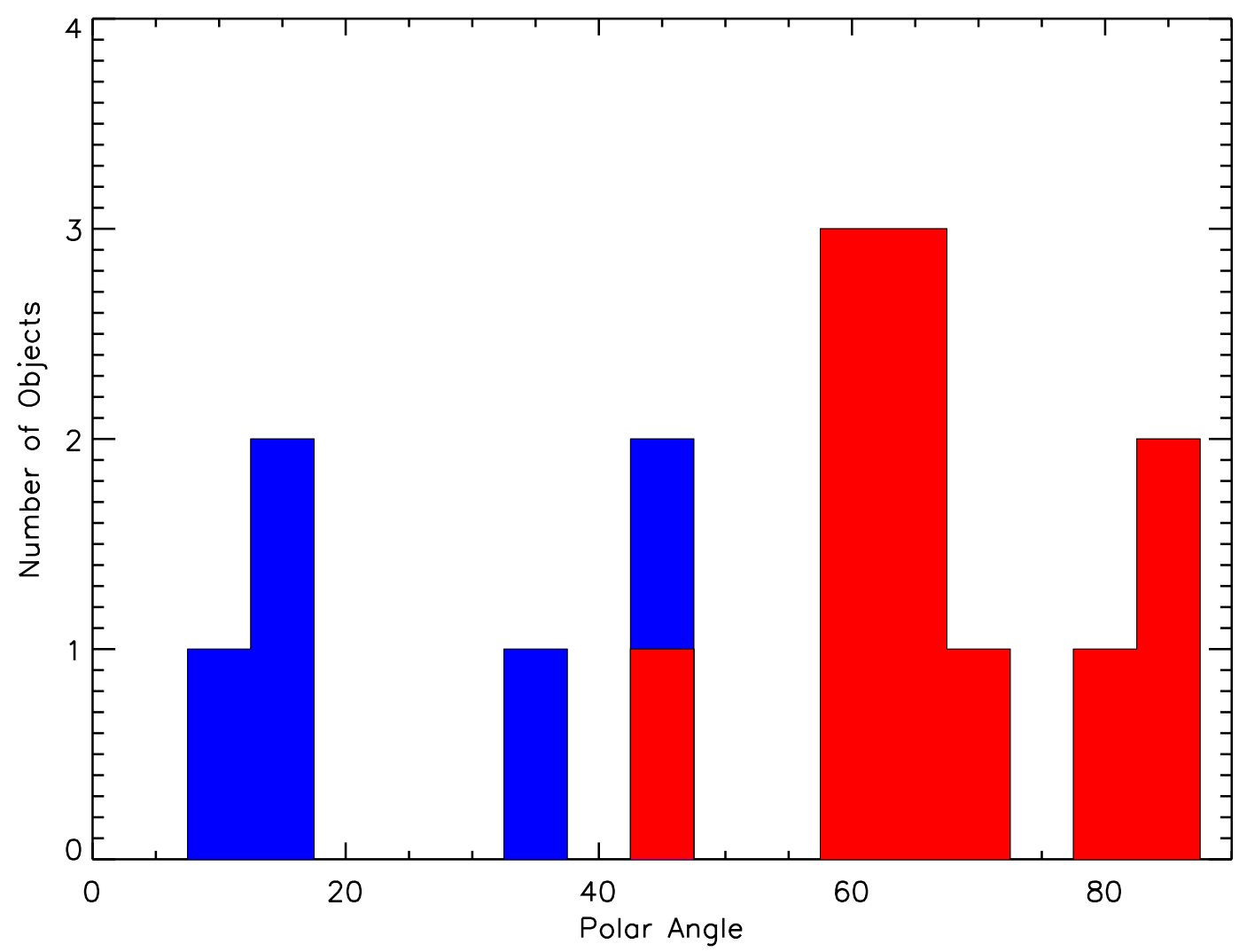

Figure 7.1 Histogram displaying the distribution of inclinations for Seyfert 1s (blue) and Seyfert 2s (red) in our sample, where the bicone axes of Seyfert 1s tend to be less inclined than Seyfert 2s.

structure, the neutral hydrogen column density $\left(\mathrm{N}_{H}\right)$ along our line of sight to the central engine increases. With our sample of inclinations and column densities from the literature, we are given the opportunity to determine the relationship between these two parameters for the first time.

For Type 1 AGN, where we assume that we are looking into the inner portion of the AGN, we do not observe large neutral hydrogen column densities representative of the material found in the torus-like structure surrounding the central engine. Instead, we typically measure ionized column densities from "AGN winds" (Crenshaw et al. 2003) to determine $N_{H}$ from photoionization models. In short, intensities of two or more resonance doublet line 


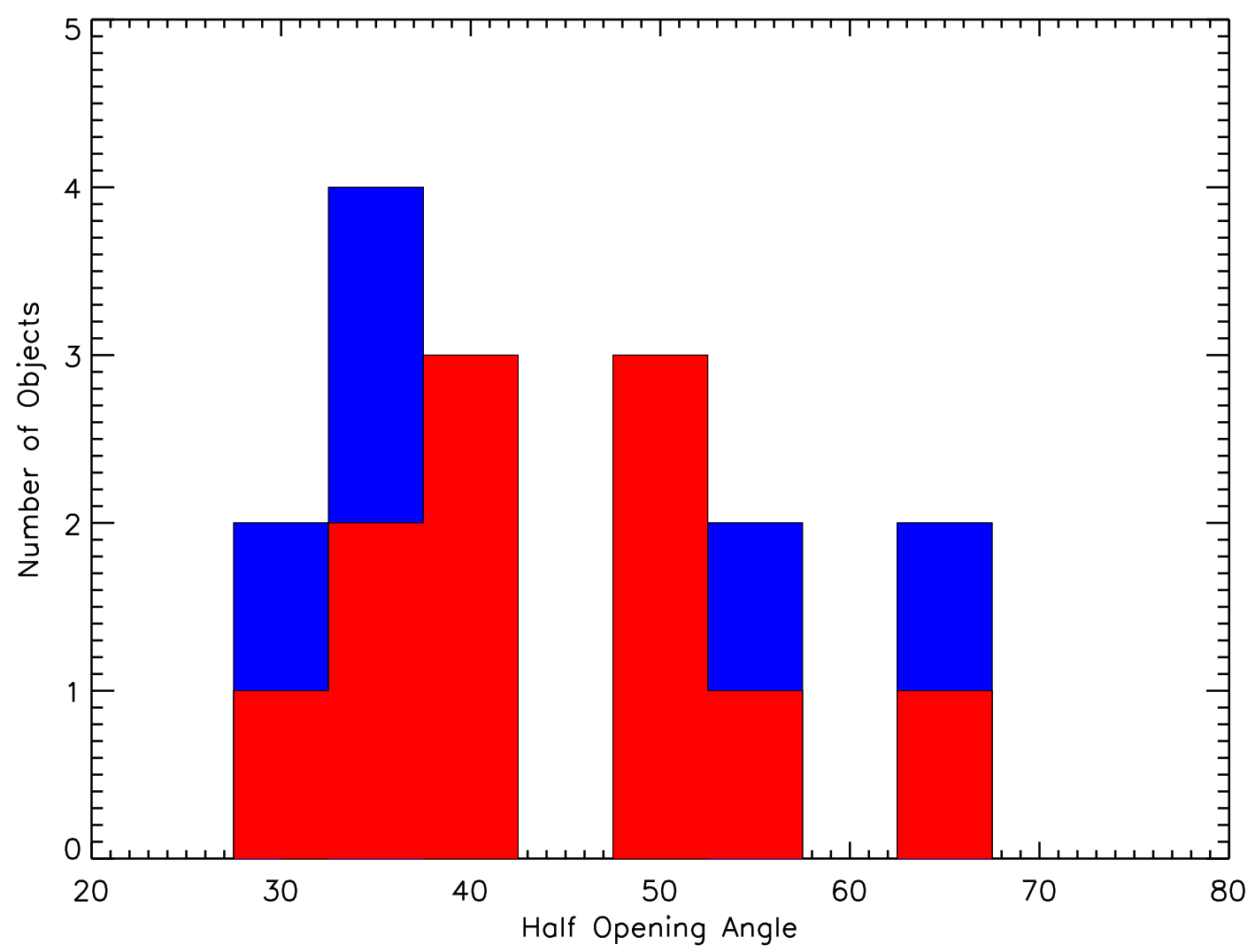

Figure 7.2 Histogram displaying the distribution of opening angles for Seyfert 1s (blue) and Seyfert 2s (red) in our sample. Seyfert 1s and Seyfert 2s appear to be evenly distributed.

species (i.e. C IV, S IV) are measured which allow for the calculation of the covering factor of the outflowing absorbers with respect to the background emission, which in turn allows for the calculation of the optical depth of each absorption feature. The ionic column density of each absorption line is then obtained by integrating the optical depth across the line profile (Crenshaw et al. 2003). These ionic column densities are used to determine the ionization parameter (ratio of ionizing photon density to gas density, $\mathrm{U})$ and $N_{H}\left(=N_{H I}+N_{H I I}\right)$ via CLOUDY photoionization modeling (Kraemer et al. 2009, 2012). $N_{H}$ values for each Sy 1 in our sample are summed column densities from individual absorption components as determined by Crenshaw \& Kraemer (2012) and references therein. 
Type 2 AGN neutral column densities are measured directly through X-ray observations. As the column density of material, containing mostly hydrogen, within the obscuring toruslike structure surrounding the AGN increases, more ionizing radiation is absorbed. This effect can be most prominently observed in the X-ray using the Chandra, XMM-Newton, and Suzaku observatories, where emission from an obscured AGN is absorbed below a specific energy due primarily to the $\mathrm{H}$ and He edges (Turner \& Miller 2009). What emission remains can be fit with an absorbed power law model using X-ray analysis software XSPEC to determine $N_{H}$ (Winter et al. 2009). All values used in this work were taken from Shu et al. (2007) and references therein. Values with lower limits are due to X-ray absorption extending to energies higher than could be observed by a specific observatory, which could therefore not be fit with a power law. In cases where the absorber is Compton thick, the lower limit is $\mathrm{N}_{H} \approx 10^{25} \mathrm{~cm}^{-2}$.

Figure 7.3 compares the column density and inclination of the bicone axis away from our line of sight for each of our modeled AGN, which suggests a distinct correlation between the two parameters. Using all plotted targets, we calculate a Pearson correlation coefficient of $r=0.78$. If we remove Mrk 279, as column densities from several its absorbers have yet to be determined (Crenshaw \& Kraemer 2012), we then calculate a coefficient of $r=0.86$. Both coefficient values correspond to a probabilty of exceeding $r$ in a random sample of observations taken from an uncorrelated parent population of $P=0.1 \%$.

As our line of sight with respect to the bicone axis (polar angle) increases, the torus surrounding the AGN becomes closer to edge-on and we see an increase in column densities for both Seyfert 1s and Seyfert 2s. Seyfert 1s and 2s can reside at similar inclinations as long as our line of sight is respectively inside or outside the NLR bicone created by the obscuring torus surrounding the AGN. Surprisingly, there appears to be a seamless transition between the Seyfert 1 ionized column densities and Seyfert 2 cold column densities, despite the supposedly different nature of these two AGN components. 


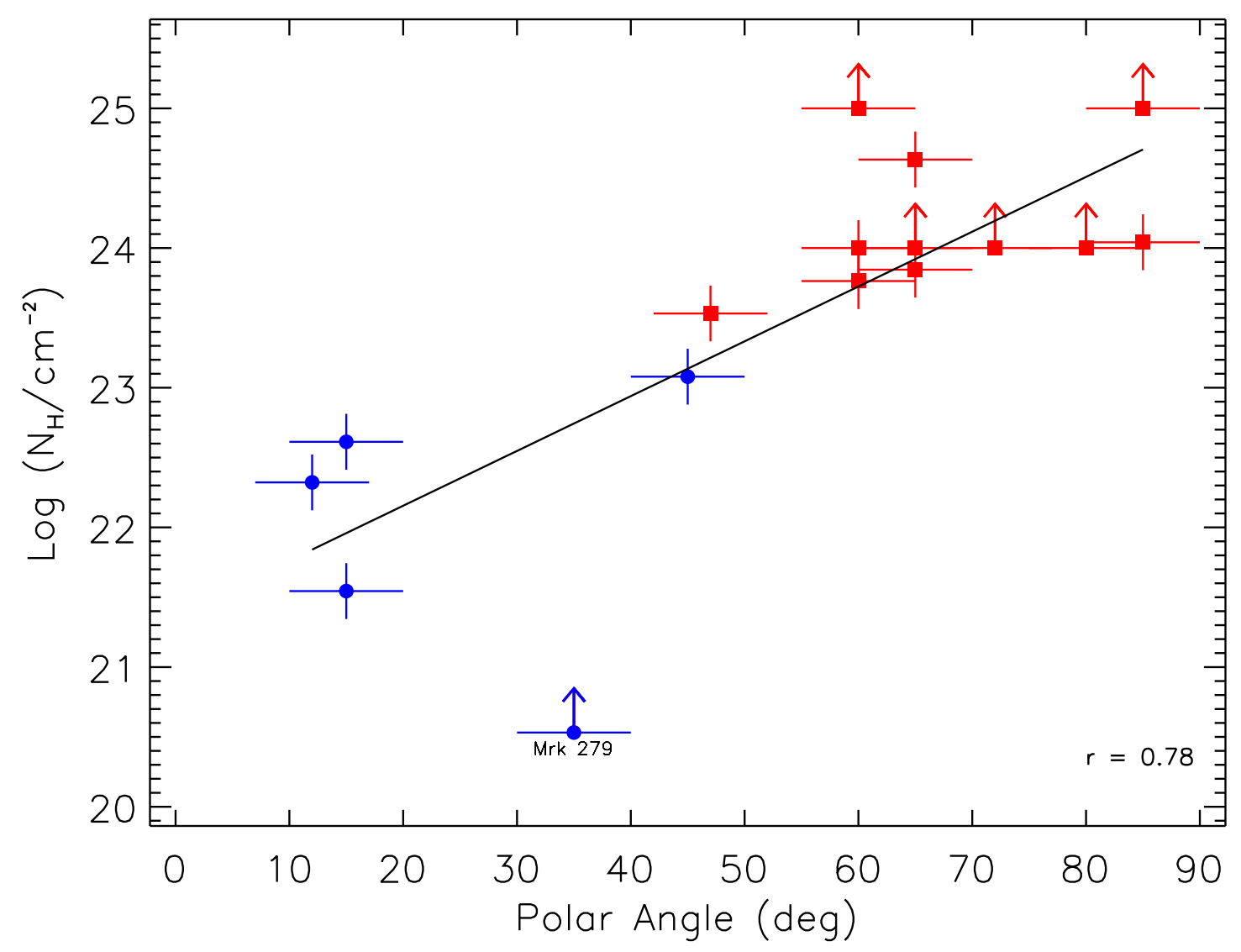

Figure 7.3 Inclination from our line of sight to the bicone axis versus the x-ray column density (ionized for Seyfert 1s, cold for Seyfert 2s), where a continuous trend can be seen between Seyfert 1s and 2s. Circles represent Sy 1s; squares represent Sy2s. Points with arrows show lower limits of column densities.

\subsection{Mid-IR Flux}

Deo et al. (2009) analyzed mid-infrared (mid-IR) spectra of Seyfert galaxies using archival Spitzer Space Telescope observations in order to characterize the nature of the mid-IR active nuclear continuum. They found that Seyfert 2 s typically have weaker short-wavelength (5.5 - $14.7 \mu \mathrm{m})$ active nuclear continua than comparable Seyfert 1s, which suggests a relationship between this mid-IR flux ratio and inclination, as short-wavelength mid-IR emission is likely absorbed to a certain degree in Type 2 objects by the obstructing torus-like structure 
surrounding the AGN. According to the unified model, as our view becomes more pole-on, the $5 \mu \mathrm{m}$ emission increases as we see more of the hot, inner throat of the torus.

Thus, we retrieved archival Spitzer spectra of our modeled sample from the Cornell Atlas of Spitzer/IRS Sources (CASSIS) (Lebouteiller et al. 2011) to compare their mid-IR color $(5 \mu \mathrm{m} / 30 \mu \mathrm{m}$ flux $)$ with their modeled inclinations. CASSIS provides reduced lowresolution spectra $(R \sim 60-127$ over $5.2 \mu \mathrm{m}$ to $38 \mu \mathrm{m})$ taken by the Infrared Spectrograph (IRS) instruments aboard Spitzer. Spectra were extracted through a dedicated pipeline from which we chose a regular "tapered" extraction suggested for extended sources. Each observation was obtained in four segments which required scaling before each segment was combined into a single spectrum. Segments were scaled such that overlapping ends of each segment were equal in flux, creating a continous spectrum when combined. As we measured relative fluxes, alteration of true flux values due to scaling were not a concern. Continuum fluxes were then measured over small bins at $5.5 \mu \mathrm{m}, 13.7 \mu \mathrm{m}, 20 \mu \mathrm{m}$, and $30 \mu \mathrm{m}$ in order to determine the mid-IR color of each AGN.

Comparing our results with Deo et al. (2009) for targets that are contained in both their sample and CASSIS (Mrk 3, NGC 4151, NGC 4507), we find our measured fluxes to be consistant to within $10 \%$. As observations of certain sample AGN were not available in CASSIS, we then use flux ratios from Deo et al. (2009) for NGC 1667, NGC 4051, and NGC 7674 .

Figure 7.5 shows a negative correlation between mid-IR color and polar angle with a correlation coefficient of $r=-0.61(P \approx 2 \%)$, indicating that a lesser polar angle allows for a better view of the hot inner 'throat' of the obscuring torus. Removing NGC 3227, a Seyfert 1 galaxy known to be heavily reddened by its host galaxy (Crenshaw \& Kraemer $2001)$, increases the correlation to $r=-0.74(P<0.5 \%)$. 


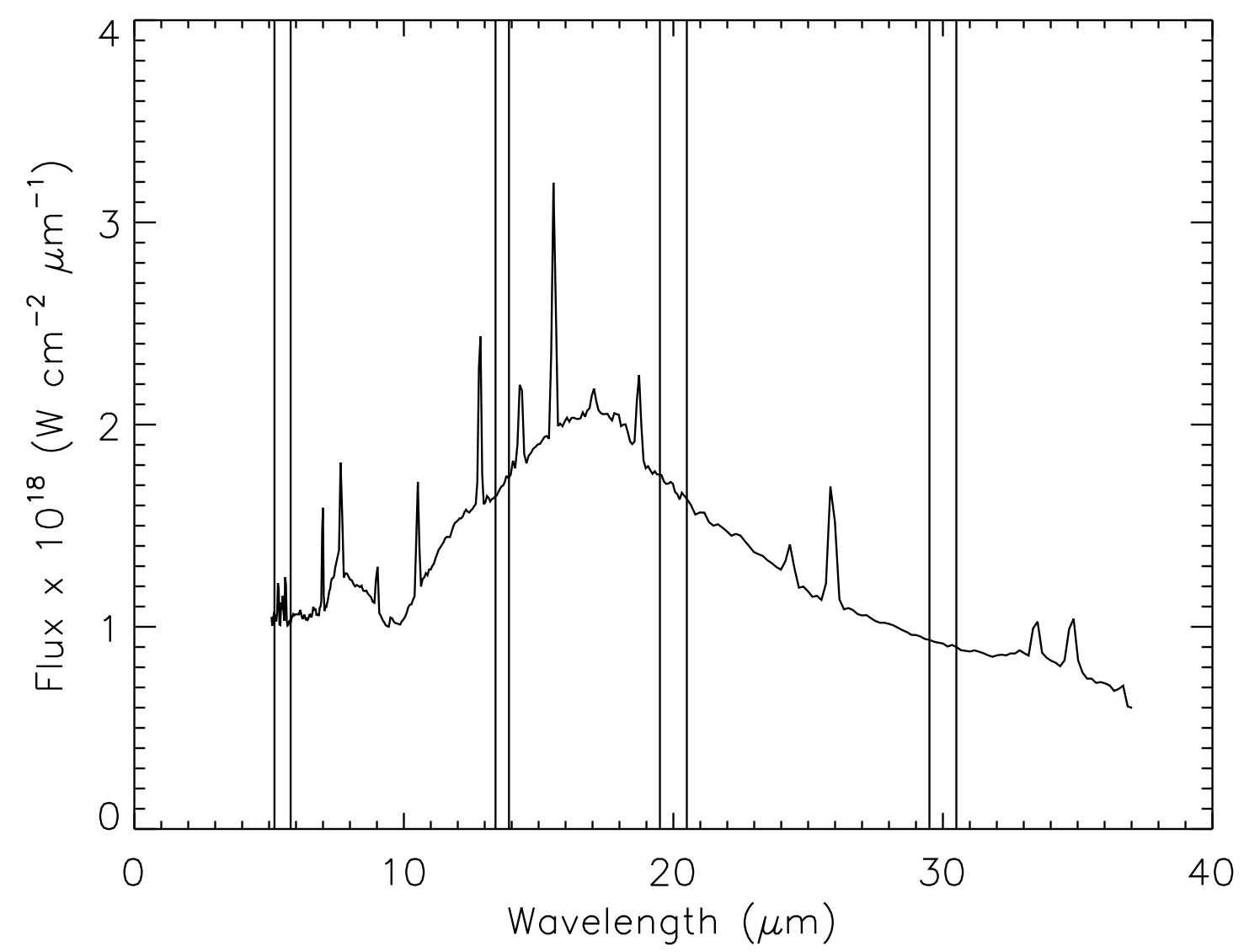

Figure 7.4 Mid-IR Spitzer spectrum of Mrk 3 gathered from CASSIS. Vertical lines from left to right denote $5.5 \mu \mathrm{m}, 13.7 \mu \mathrm{m}, 20 \mu \mathrm{m}$, and $30 \mu \mathrm{m}$ flux bins.

\section{$7.3 \mathrm{H} \beta$ FWHM}

By observing the FWHM of broad $\mathrm{H} \beta$ emission-lines in Sy 1 targets and comparing them with their modeled inclinations, we can gain a better understanding of the BLR kinematics.

We obtained FWHM from the literature except for two Seyferts and so we obtained spectra for them. Observations for NGC 3227 were obtained using the DeVeny spectrograph on the $1.8 \mathrm{~m}$ Perkins telescope at the Lowell Observatory in Flagstaff, Arizona. Spectra were taken 2010 December 9 using a slit width of $2^{\prime \prime}$ for a total exposure of 1200s and dispersed into spectral images using the 'blue' 300 line $\mathrm{mm}^{-1}$ grating with a resolving power of $\sim 2145$ 
Table 7.2 Measured Mid-IR fluxes of the modeled AGN sample.

\begin{tabular}{lcc}
\hline Target & $\begin{array}{c}\mathrm{F}_{5.5 \mu m} \\
\left(\mathrm{~W} \mathrm{~cm}^{-2} \mu \mathrm{m}^{-1}\right)\end{array}$ & $\begin{array}{c}\mathrm{F}_{30 \mu m} \\
\left.\mathrm{~cm}^{-2} \mu \mathrm{m}^{-1}\right)\end{array}$ \\
\hline This Work & & \\
Mrk 3 & $1.08 \times 10^{-18}$ & $8.96 \times 10^{-19}$ \\
Mrk 34 & $2.75 \times 10^{-19}$ & $1.85 \times 10^{-19}$ \\
Mrk 78 & $3.53 \times 10^{-19}$ & $2.10 \times 10^{-19}$ \\
Mrk 279 & $7.47 \times 10^{-19}$ & $1.35 \times 10^{-19}$ \\
Mrk 573 & $7.45 \times 10^{-19}$ & $2.58 \times 10^{-19}$ \\
Mrk 1066 & $7.45 \times 10^{-19}$ & $1.10 \times 10^{-18}$ \\
NGC 3227 & $1.42 \times 10^{-18}$ & $6.52 \times 10^{-19}$ \\
NGC 3783 & $2.37 \times 10^{-18}$ & $7.07 \times 10^{-19}$ \\
NGC 4151 & $6.23 \times 10^{-18}$ & $1.39 \times 10^{-18}$ \\
NGC 4507 & $2.09 \times 10^{-18}$ & $5.50 \times 10^{-19}$ \\
NGC 5643 & $4.73 \times 10^{-19}$ & $1.01 \times 10^{-18}$ \\
Deo et al. (2009) & & \\
NGC 1667 & $7.50 \times 10^{-19}$ & $2.22 \times 10^{-19}$ \\
NGC 4051 & $2.09 \times 10^{-18}$ & $4.13 \times 10^{-19}$ \\
NGC 7674 & $1.87 \times 10^{-18}$ & $6.10 \times 10^{-19}$ \\
\hline
\end{tabular}

$\left(\sim 140 \mathrm{~km} \mathrm{~s}^{-1}\right)$.

Observations for NGC 3783 was obtained using the R-C spectrograph on the 1.5 SMARTS telescope at the Cerro Tololo Inter-American Observatory (CTIO) in La Serena, Chile. Longslit spectra were taken 2003 April 25 for a total exposure time of 1800s using the 600 line $\mathrm{mm}^{-1}$ '\#26' grating with a resolving power of $\sim 1165\left(\sim 257 \mathrm{~km} \mathrm{~s}^{-1}\right)$.

Spectra for both observations were reduced two-dimensionally and collapsed to a single spectrum using IRAF, resulting in wavelength calibrated spectra in flux units from which FWHMs could be measured.

Figure 7.6 shows a positive correlation $(r=0.94, P<2 \%)$ between FWHM and polar angle, which suggests a non-spherical component to the BLR kinematics. If the BLR kinematics are due to a strong rotational component, as suggested by a number of studies (Gaskell 2000), this correlation suggests that pole-on and near pole-on AGN contain ve- 


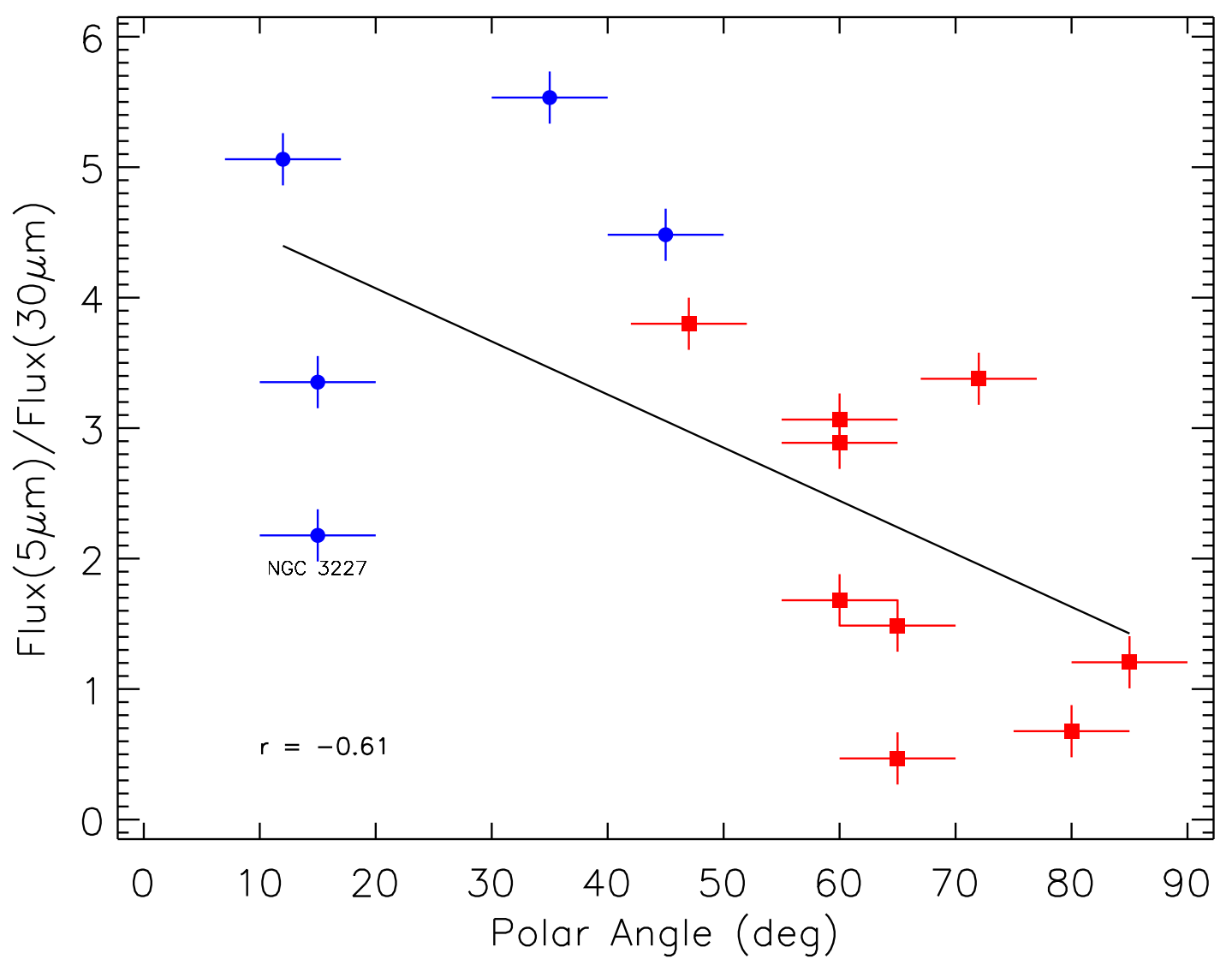

Figure 7.5 Inclination from our line of sight to the bicone axis versus AGN mid-IR color $(5 \mu \mathrm{m} / 30 \mu \mathrm{m}$ flux $)$. Circles represent Sy 1s; squares represent Sy2s.

locities that are underestimated. Thus, using the mass relation via reverberation mapping (Peterson 1997),

$$
M=f \frac{R \Delta V^{2}}{G}
$$

where $R$ is the size of the BLR, $\Delta V$ is the emission line width, and $f$ is a scale factor of order unity dependant on the inclination, amongst other things, of the BLR, the correlation also suggests that black-hole masses may be underestimated in some AGN. For example, using the measured mass, assuming a scale factor, $f$, of 5.5 (Collin et al. 2006; Denney 


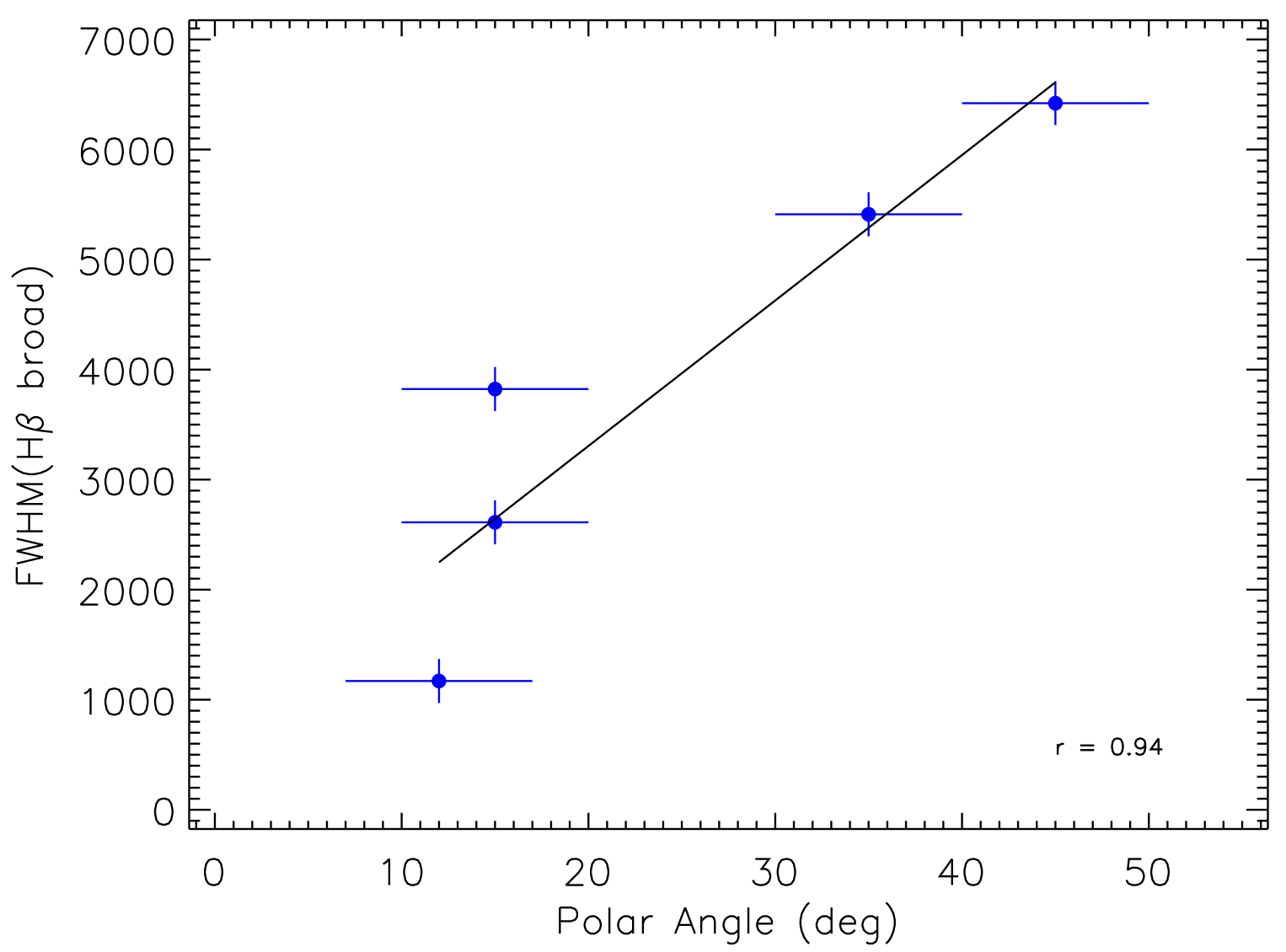

Figure 7.6 Inclination from our line of sight to the bicone axis versus H $\beta$ FWHM for modeled Seyfert 1s.

et al. 2010), and inclination modeled for NGC 4051 of $1.73 \times 10^{6} M \odot$ and $12^{\circ}$ respectively and assuming full dependence of the FWHM on rotation, we can calculate a deprojected $\mathrm{H} \beta$ FWHM of $\approx 5600 \mathrm{~km} \mathrm{~s}^{-1}$ and a deprojected $\mathrm{M}_{B H} \approx 3.96 \times 10^{7} M_{\odot}$. Furthermore, increased FWHM and thus mass would show that current values for AGN Eddington ratios are overestimated as $L / L_{E d d} \propto L / M$, this effect would decrease the Eddington ratio of NGC 4051 from $L / L_{E d d}=.17$ to $L / L_{E d d}=.0074$. 


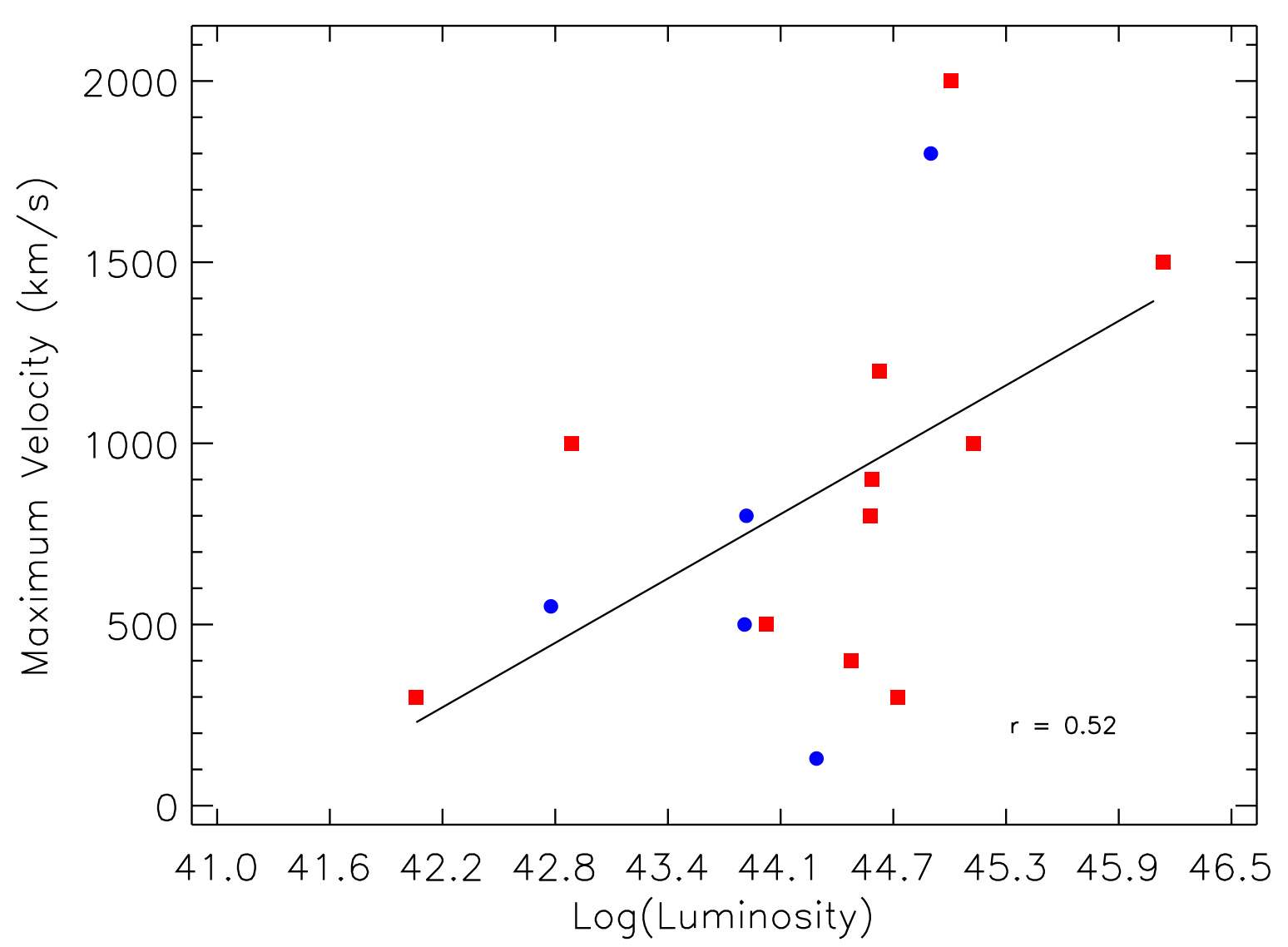

Figure 7.7 Bolometric luminosity versus maximum deprojected outflow velocity of the NLR for each of our modeled targets. Seyfert 1s are designated as blue circles, Seyfert 2s are designated as red squares.

\subsection{Possible Correlations}

Figure 7.7 suggests that as the AGN increases in luminosity, the maximum outflow velocity in the NLR also increases. This trend $(r=0.52, P<5 \%)$ supports AGN driven outflow as the source of the observed kinematics, though it remains unclear where the outflowing gas originates.

Figure 7.8 suggests a dependance of luminosity on the height of the NLR bicone. While there is not a distinct correlation between these two parameters $(r=0.43, P \approx 10 \%)$, an 


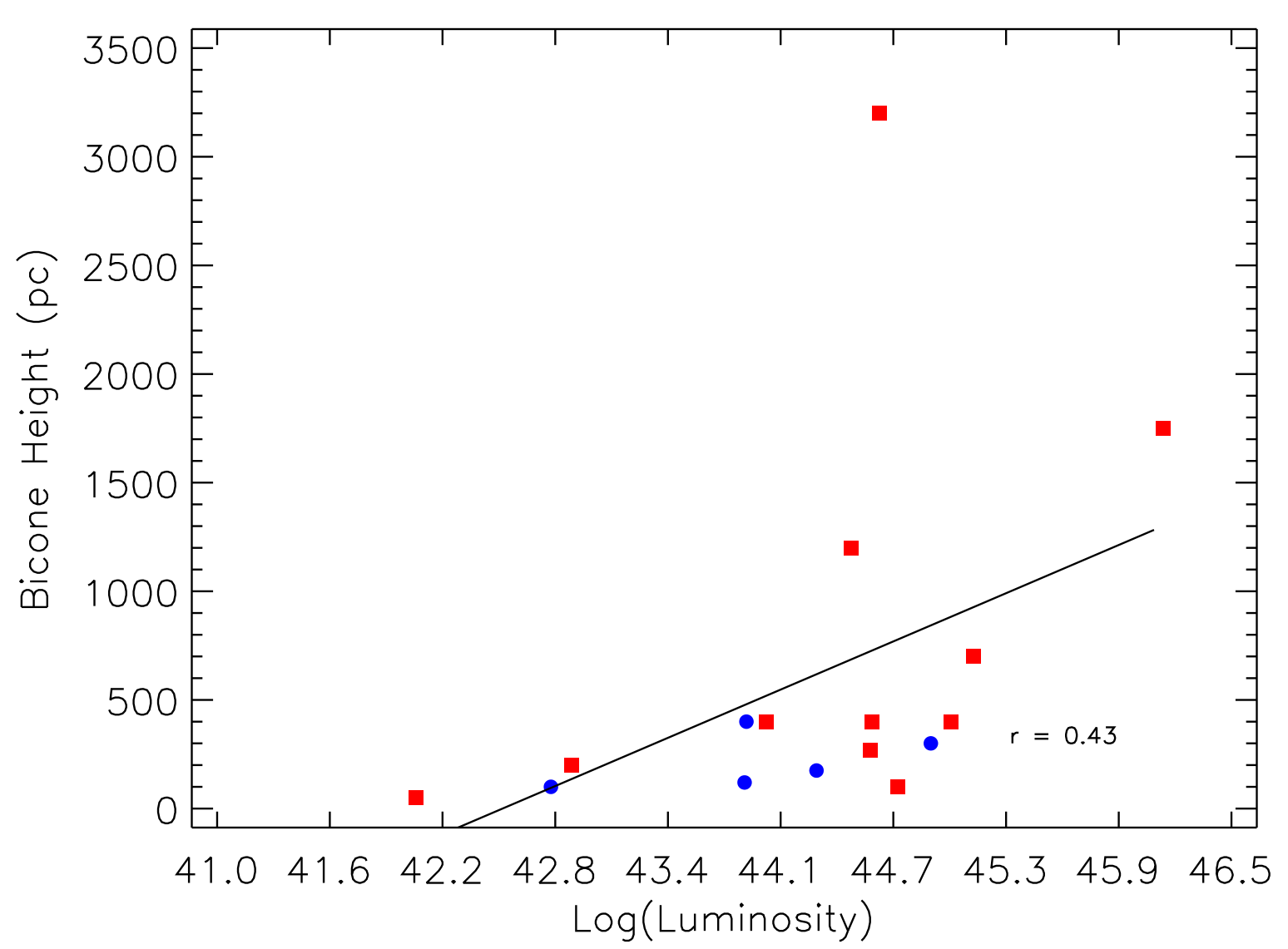

Figure 7.8 Bolometric luminosity versus bicone height for each of our modeled targets. Seyfert $1 \mathrm{~s}$ are designated as blue circles, Seyfert $2 \mathrm{~s}$ are designated as red squares.

upper limit on height can be established out to $\mathrm{L}_{B o l} \approx 44.5 \operatorname{ergs~s}^{-1}$ where Mrk 78 resides with a bicone height of $z=3200$ pc.

\subsection{Non-Correlations}

While the observable parameters above appear to be correlated with inclination, other relations we thought might exist are not found. Correlations of bolometric luminosity $\left(\mathrm{L}_{b o l}\right)$ versus inclination and outer opening angle and black hole mass versus maximum outflow velocity show no relation between each parameter. These results are not to say that such correlations do not exist, but that each correlation may be more complex than comparing two 
parameters or that we do not have enough dynamic range. For example, though we see no relation between luminosity and opening angle, where one may assume increased radiation pressure would force the opening angle of the NLR into a wider geometry, our comparison does not account for the varied amount of material surrounding each AGN, with more dense surroundings possibly constraining NLR to smaller opening angles.

\subsection{Discussion}

In targets Mrk 279 and NGC 3227, our model, when paired with the host disk, depict NLRs that are nearly bisected by the disk. While the resulting wedge geometry of the intersection matches well with the imaging, our model assumes that the inner portion of each NLR cone is hollow. Why then, do these two targets not contain any velocities that would be expected for a filled cone? Understanding this discrepency between model and observation will lead to a more sophisticated outflow model.

Many of the observed NLRs in imaging are intersections between the NLR ionizing radiation and the host disk and are projections of the true opening angle of each bicone per our models. It can be assumed for these targets that the emitting gas did not originate in the nucleus of the AGN, but rather it was accelerated off the host disk. Does any NLR gas originate in the nucleus or is it mainly in situ acceleration? Perhaps the NLR results from a stream of unquenched ionizing radiation unleashed upon the galaxy, ionizing and accelerating material that it intersects? The NLR in Circinus, which is near perpendicular to the host galaxy, is only $50 \mathrm{pc}$ in height. As our own Milky Way has a scale height of less than $100 \mathrm{pc}$, could we be seeing the matter-bounded ionization of an abnormally rich (Freeman et al. 1977) host disk above the AGN?

While determining inclinations for 16 Seyfert AGN is encouraging, a large mystery now presents itself as to why we cannot model the remaining 41 AGN in our sample. The answer is clear for AGN with compact NLRs: with high velocity components of these NLR observed 
in less than a $0.5^{\prime \prime}$ diameter over the nucleus, we simply do not have the resolution to observe individual kinematic components. To better understand the 17 ambiguous kinematics AGN, developing a rotational kinematic model to fit the data would be the most simple way to proceed. After fitting and removing all AGN exhibiting rotational kinematics, we can begin to search for the truly absent outflow components in the remaining AGN. As these data already exist, improving our models will be the most effective way to illuminate AGN physics moving forward without needing additional observations.

Osterbrock \& Shaw (1988) used statistics of numbers of Seyfert 1, 1.5, 1.8, 1.9, and 2 galaxies in Wasilewski field to find that $78 \%$ of field AGN were type 2, leading them to calculate an average torus half-opening angle of approximately $35^{\circ}$. From our sample, we find that the average opening angle of $44^{\circ}$ is bigger than this calculated opening angle. Schmitt et al. (2001) calculated a half opening angle of $48^{\circ}$, a value similar to our results, by binning Seyfert $1.8 \mathrm{~s}$ and $1.9 \mathrm{~s}$ with Seyfert $2 \mathrm{~s}$ to determine the ratio of Seyfert $1 \mathrm{~s}$ to $2 \mathrm{~s}$. With values consistently above those determined from the Seyfert 1 / Seyfert 2 ratio, we must question if simple obscuration by tori explains the relative fraction of Seyfert 1s to Seyfert 2s. For the opening angle to be so large and depend solely on the torus for its definition, the estimated percentage of Seyfert 2 s observed would be less than what we actually see. It is possible that some secondary property could be accountable for the fraction of Seyfert $2 \mathrm{~s}$ that we see, such as the presence of obscuring gas in the host disk (i.e. NGC 5506) which is separate from the torus, although possibly connected to the creation of dusty circumnuclear gas that makes a torus.

The most important correlation discovered is likely the relation between inclination and column density because it provides extension of the unified model to indicate a continuous variation of gas column density as a function of viewing angle, thus revealing the structure of the circumnuclear regions in AGN. Interestingly, Seyfert 1s and 2s can reside at similar inclinations as long as our line of sight is respectively inside or outside the NLR. Surprisingly, 
there appears to be a seamless transition between the Seyfert 1 ionized column densities and Seyfert 2 cold column densities suggesting a connection between the obscuring matter and the outflowing ionized winds.

Observing the relation between bolometric luminosity and maximum outflow velocity, is it possible that outflow kinematics cease at $\mathrm{L}_{b o l}<10^{41} \mathrm{erg} \mathrm{s}^{-1}$ ? HST STIS H $\alpha$ studies similar to our own in Walsh et al. (2008) depict observed low-ionization nuclear emissionline regions (LINERs), AGN with luminosities generally lower than Seyferts, containing 'ambiguous' kinematics with blueshifted velocities $<250 \mathrm{~km} \mathrm{~s}^{-1}$. Though these velocities may be due to projected outflows, they could instead be a combination of rotation and ionization via star formation or post-AGB stars (Yan \& Blanton 2012).

With accurate, continuous correlations between AGN inclination and several independant, observable parameters, it may soon be possible to use these comparitively simple to obtain observational measurements as a proxy for inclination in AGN with unmodelable kinematics.

With evidence of inclination dependant correlations, what other observable parameters could be related to inclination? Could the amount of polarized light depend on inclination? As Antonucci (1993) observed, polarization of type 1 galaxies tends to be parallel to the AGN jet axis and polarization of type 2 galaxies tends to be perpendicular to the jet axis. Could this also apply to the AGN itself, such that a correlation between increasing perpendicular polarization and polar angle exists?

This relation could also be studied by analyzing AGN radio jets and how they correlate with inclination when they are present. [O III] imaging overplotted with radio jet axes in Schmitt et al. (2003) show that they are usually oriented in the same direction. We can compare the position angle of the radio axis with the actual position angle of the NLR determined by our models, instead of apparent position angle seen in imaging, a difference often due to an intersection between the ionizing bicone and host disk as mentioned above. 


\section{CHAPTER 8}

\section{CONCLUSIONS}

\subsection{Lessons Learned}

\subsubsection{In Situ Acceleration in NLRs}

We determined the inclination of the AGN in Mrk 573 and found that the bicone of ionizing radiation from the AGN intersects with the host disk, illuminating a section of the disk including inner segments of spiral arms. Combining the kinematic data with [O III] image analysis suggests that the peculiar velocities are either due to rotation or, more likely, in situ acceleration of gas off the previously non-emitting inner dust/gas spiral arms. The latter case more easily explains the large velocity dispersions at each emission arc. Given the large mass outflow rates likely present in NLRs (Collins et al. 2009), it is likely that in situ acceleration is widely important.

\subsubsection{Double Peaked Emission Lines Do Not Necessarily Equal Double AGN}

We employed a similar method to determine the inclination of the AGN in Mrk 78 and found that the double peaked emission lines seen in ground-based spectra of Mrk 78 are due to an asymmetric distribution of outflowing gas in the NLR and were able to fit a single outflow model to the kinematics of Mrk 78. This provides strong evidence against the use of double narrow emission lines in AGN spectra alone as a signature for dual AGN, as we showed it is possible that many double peaked profiles may be due to outflows in a single AGN.

\subsubsection{Inclination Dependent AGN Properties}

We measured radial velocities across NLRs for all remaining Seyfert AGN observed with HST/STIS G430L/M gratings. From our measurements, we found an additional 11 AGN that contained kinematics characteristic of biconical outflow. Biconical outflow may still exist 
in the remaining targets, though the required kinematic components are not currently visible because they may be too compact or faint. Similar to observations of Mrk 3 and Mrk 573, NLR emission of many modeled targets comes from the intersection between host disk and ionizing radiation bicone, which suggests that NLRs may more often ionize and accelerate the medium they contact rather than accelerate clouds of material from the nucleus.

With inclinations and geometries of 16 Seyfert galaxies, our model results agree with the unified model in that Seyfert 1 AGN are inclined futher toward our LOS than Seyfert 2 AGN. Knowing the inclinations of these AGN also allows us to determine how their observed properties vary as a function of polar angle with respect to the accretion disk and/or torus axes. We have established a strong connection between column density and polar angle, which probes the structure of the obscuring torus, the ionized gas outflows, and the connection between the two. We have also determined correlations between polar angle and FWHM (broad $\mathrm{H} \beta$ ), suggesting a non-spherical component to the BLR kinematics, and increasing mid-IR color ( $5 \mu \mathrm{m} / 30 \mu \mathrm{m}$ flux) with decreasing polar angle, presumably indicating a better view of the hot inner "throat" of the obscuring torus.

With the discovery of three independant correlations on inclination, we can see that our line of sight likely affects how we observe specific parameters of our sample and thus that the obstructing torus-like structure surrounding the central engine plays a significant role in how we observe these AGN. Alternatively, we find little correlation between SMBH characteristics and the NLR kinematics and geometry, an observation that may be due to the small range in mass and luminosity within our sample. Nonetheless, this suggests that the mechanics behind outflowing systems in AGN rely not only on the central SMBH, but the environment surrounding them. 


\subsection{Future Work}

Data for additional AGN will help to confirm and clarify the connection between inclination and column density, as well as test the significance and quantify the exact relationships of the latter correlations. As mentioned earlier, our technique for determining inclinations of AGN is not a simple task and correlating inclination with parameters that are more easily observable could also provide astronomers a way to estimate the inclination of an AGN without modeling its NLR kinematics. Thus, the motivation for future study then is to expand beyond our current results by 1) determining the inclinations of the NLRs in a much larger sample of AGN, and 2) determining the multiwavelength properties of these AGN to test the above correlations and identify others that will probe the structure of the AGN components.

\subsubsection{Increase the Sample of AGN Inclinations}

Recent works that produce outflow models similar to our own (Storchi-Bergmann et al. 2010; Müller-Sánchez et al. 2011) include infrared IFU observations, as using multiple HST/STIS long-slit observations to map out the NLR and surrounding areas would prove inefficient. Aside from the ability to map out the entire NLR, infrared observations allows us to analyze the kinematics of the [S III] $0.9533 \mu \mathrm{m}$ emitting gas, which originates in the same gas that emits [O III] observable in HST long-slit optical spectra, avoiding extinction problems present at optical wavelengths and allowing us to observe the full extent of the biconical outflow. IFU studies can begin immediately as we have already received Z band observations (which

include [S III] $0.9533 \mu \mathrm{m}$ emission) for Mrk 3 and Mrk 573 using Gemini NIFS through a proposal by D.M. Crenshaw (we are exploring the NIFS archives for additional Z-band AGN observations). We will soon receive observations taken with the Keck OSIRIS IFU through a proposal by M. Malkan that will attempt to model the NLR in a similarly minded project 
by measuring narrow $\operatorname{Br} \gamma$ emission in the K-band.

In addition to mapping NLR emission lines, infrared IFU observations also contain additional emission and absorption lines that provide supplementary information about the AGN. K-band observations contain $\mathrm{H}_{2} 2.1218 \mu \mathrm{m}$ emission (see Storchi- Bergmann et al. 2009) that reveals the kinematics of the warm molecular gas and possible fueling flows close to the nucleus. If more than one $\mathrm{H}_{2}$ emission line is detected, the $\mathrm{H}_{2}$ temperature can be calculated. The $\mathrm{K}$ band also includes the $\mathrm{CO}$ stellar absorption bands which can be used to derive the stellar kinematics and assess the component of the gas kinematics due to gravitational motion in the central potential of the galaxy. Analysis of these additional lines could be done in conjunction with NLR emission lines.

We will propose for additional Gemini NIFS observations of new targets which would be drawn from the Revised Shapley Ames (RSA) and Ho Palomar flux limited galaxy samples, which do not bias against AGN type or property and span over five orders of magnitude in luminosities and a wide range in black hole masses and Eddington ratios. We will also propose for observations of AGN present in our full, 52 target sample that display signs of rotation and/or high velocity kinematics currently too complex for our current outflow model. A rotation component will then be added to our current model to isolate effects of rotation from outflows, as cases exist where both components are important, to determine the geometry of the latter.

Additionally, we will continue proposing for HST observations of AGN, focusing on those with recent [O III] imaging (requiring only STIS spectra, thus reducing the amount of HST orbits requested by half) and AGN that cover a range of column densities not probed by currently modeled AGN. As we can currently go no further analyzing Seyfert STIS [O III] spectra, having exhausted the archive database, we will also return to the HST archive to include QSOs that exhibit extended emission in order to expand our sample. 


\subsubsection{Determine the Multiwavelength Properties of the AGN Sample}

In addition to expanding our sample, we will also need accurate values for $\mathrm{N}_{H}, \mathrm{H} \beta \mathrm{FWHM}$, SEDs, and other AGN parameters. After a thorough literature search looking for previously measured values, we will identify archive observations that can be used for new measurements. Spitzer will be used to determine infrared colors and X-ray missions for column densities. Georgia State possesses its own optical database from Lowell and Cerro Tololo Inter-American Observatories useful for $\mathrm{H} \beta$ FWHM. In addition, we will also propose for new observations, specifically using Suzaku to get high column densities of Compton-thick Seyfert 2 s that will allow us to replace limits in the current sample with actual values, HST/Chandra/XMM for ionized absorption column densities, and ground-based observations for line widths. 


\section{REFERENCES}

Adams, T. F. 1973, ApJ, 179, 417

Afanasiev, V. L., Burenkov, A. N., Shapovalova, A. I., \& Vlasyuk, V. V. 1996, in Astronomical Society of the Pacific Conference Series, Vol. 91, IAU Colloq. 157: Barred Galaxies, ed. R. Buta, D. A. Crocker, \& B. G. Elmegreen, 218

Alonso-Herrero, A. et al. 2011, ApJ, 736, 82

Antonucci, R. 1993, ARA\&A, 31, 473

Antonucci, R. R. J., \& Miller, J. S. 1985, ApJ, 297, 621

Arribas, S., Mediavilla, E., \& Garcia-Lorenzo, B. 1996, ApJ, 463, 509

Barbosa, F. K. B., Storchi-Bergmann, T., Cid Fernandes, R., Winge, C., \& Schmitt, H. 2009, MNRAS, 396, 2

Bentz, M. C. et al. 2006, ApJ, 651, 775

Bian, W., \& Gu, Q. 2007, ApJ, 657, 159

Bower, G., Wilson, A., Morse, J. A., Gelderman, R., Whittle, M., \& Mulchaey, J. 1995, ApJ, 454, 106

Buta, R. J., Byrd, G. G., \& Freeman, T. 2003, AJ, 125, 634

Christopoulou, P. E., Holloway, A. J., Steffen, W., Mundell, C. G., Thean, A. H. C., Goudis, C. D., Meaburn, J., \& Pedlar, A. 1997, MNRAS, 284, 385

Collin, S., Kawaguchi, T., Peterson, B. M., \& Vestergaard, M. 2006, A\&A, 456, 75

Collins, N. R., Kraemer, S. B., Crenshaw, D. M., Bruhweiler, F. C., \& Meléndez, M. 2009, ApJ, 694, 765

Collins, N. R., Kraemer, S. B., Crenshaw, D. M., Ruiz, J., Deo, R., \& Bruhweiler, F. C. 2005, ApJ, 619, 116

Crenshaw, D. M., \& Kraemer, S. B. 2000, ApJ, 532, L101

—. 2001, ApJ, 562, L29 
—. 2005, ApJ, 625, 680

—. 2007, ApJ, 659, 250

—. 2012, ApJ, 753, 75

Crenshaw, D. M., Kraemer, S. B., \& George, I. M. 2003, ARA\&A, 41, 117

Crenshaw, D. M. et al. 2000, AJ, 120, 1731

Crenshaw, D. M., Kraemer, S. B., Schmitt, H. R., Jaffé, Y. L., Deo, R. P., Collins, N. R., \& Fischer, T. C. 2010a, AJ, 139, 871

Crenshaw, D. M. et al. 2002, ApJ, 566, 187

Crenshaw, D. M., Schmitt, H. R., Kraemer, S. B., Mushotzky, R. F., \& Dunn, J. P. 2010b, ApJ, 708, 419

Dahari, O., \& De Robertis, M. M. 1988, ApJ, 331, 727

Das, V. et al. 2005, AJ, 130, 945

Das, V., Crenshaw, D. M., \& Kraemer, S. B. 2007, ApJ, 656, 699

Das, V., Crenshaw, D. M., Kraemer, S. B., \& Deo, R. P. 2006, AJ, 132, 620

De Robertis, M. M., \& Shaw, R. A. 1990, ApJ, 348, 421

de Vaucouleurs, G. 1958, ApJ, 127, 487

Denney, K. D. et al. 2010, ApJ, 721, 715

—. 2009, ApJ, 702, 1353

Deo, R. P., Crenshaw, D. M., \& Kraemer, S. B. 2006, AJ, 132, 321

Deo, R. P., Richards, G. T., Crenshaw, D. M., \& Kraemer, S. B. 2009, ApJ, 705, 14

Elitzur, M., \& Shlosman, I. 2006, ApJ, 648, L101

Erkens, U., Appenzeller, I., \& Wagner, S. 1997, A\&A, 323, 707

Fabbiano, G., Wang, J., Elvis, M., \& Risaliti, G. 2011, Nature, 477, 431

Fischer, T. C., Crenshaw, D. M., Kraemer, S. B., Schmitt, H. R., Mushotsky, R. F., \& Dunn, J. P. 2011, ApJ, 727, 71 
Fischer, T. C., Crenshaw, D. M., Kraemer, S. B., Schmitt, H. R., \& Trippe, M. L. 2010, AJ, 140,577

Freeman, K. C., Karlsson, B., Lynga, G., Burrell, J. F., van Woerden, H., Goss, W. M., \& Mebold, U. 1977, A\&A, 55, 445

Gaskell, C. M. 2000, New A Rev., 44, 563

Greenhill, L. J. et al. 2003, ApJ, 590, 162

Grupe, D., Wills, B. J., Leighly, K. M., \& Meusinger, H. 2004, AJ, 127, 156

Gu, Q., Melnick, J., Cid Fernandes, R., Kunth, D., Terlevich, E., \& Terlevich, R. 2006, MNRAS, 366, 480

Heckman, T. M., Ptak, A., Hornschemeier, A., \& Kauffmann, G. 2005, ApJ, 634, 161

Hicks, E. K. S., Davies, R. I., Malkan, M. A., Genzel, R., Tacconi, L. J., Müller Sánchez, F., \& Sternberg, A. 2009, ApJ, 696, 448

Hutchings, J. B. et al. 1998, ApJ, 492, L115

Khachikian, E. Y., \& Weedman, D. W. 1974, ApJ, 192, 581

Kinney, A. L., Antonucci, R. R. J., Ward, M. J., Wilson, A. S., \& Whittle, M. 1991, ApJ, 377,100

Kinney, A. L., Schmitt, H. R., Clarke, C. J., Pringle, J. E., Ulvestad, J. S., \& Antonucci, R. R. J. 2000, ApJ, 537, 152

Kondratko, P. T., Greenhill, L. J., \& Moran, J. M. 2006, ApJ, 652, 136

Koski, A. T. 1978, ApJ, 223, 56

Kraemer, S. B., \& Crenshaw, D. M. 2000a, ApJ, 532, 256

—. 2000b, ApJ, 544, 763

Kraemer, S. B. et al. 2012, ApJ, 751, 84

Kraemer, S. B., Crenshaw, D. M., Hutchings, J. B., Gull, T. R., Kaiser, M. E., Nelson, C. H., \& Weistrop, D. 2000, ApJ, 531, 278 
Kraemer, S. B., Trippe, M. L., Crenshaw, D. M., Meléndez, M., Schmitt, H. R., \& Fischer, T. C. 2009, ApJ, 698, 106

Lawrence, A., \& Elvis, M. 2010, ApJ, 714, 561

Lebouteiller, V., Barry, D. J., Spoon, H. W. W., Bernard-Salas, J., Sloan, G. C., Houck, J. R., \& Weedman, D. W. 2011, ApJS, 196, 8

Markowitz, A., Reeves, J. N., George, I. M., Braito, V., Smith, R., Vaughan, S., Arévalo, P., \& Tombesi, F. 2009, ApJ, 691, 922

Meléndez, M., Kraemer, S. B., Schmitt, H. R., Crenshaw, D. M., Deo, R. P., Mushotzky, R. F., \& Bruhweiler, F. C. 2008, ApJ, 689, 95

Morris, S., Ward, M., Whittle, M., Wilson, A. S., \& Taylor, K. 1985, MNRAS, 216, 193

Müller-Sánchez, F., Prieto, M. A., Hicks, E. K. S., Vives-Arias, H., Davies, R. I., Malkan, M., Tacconi, L. J., \& Genzel, R. 2011, ApJ, 739, 69

Mundell, C. G., Wrobel, J. M., Pedlar, A., \& Gallimore, J. F. 2003, ApJ, 583, 192

Nagao, T., Kawabata, K. S., Murayama, T., Ohyama, Y., Taniguchi, Y., Sumiya, R., \& Sasaki, S. S. 2004, AJ, 128, 109

Nelson, C. H., \& Whittle, M. 1995, ApJS, 99, 67

Osterbrock, D. E. 1978, Proceedings of the National Academy of Science, 75, 540

Osterbrock, D. E., \& Shaw, R. A. 1988, ApJ, 327, 89

Peterson, B. M. 1997, An Introduction to Active Galactic Nuclei (Cambridge University Press)

Peterson, B. M. et al. 2004, ApJ, 613, 682

Pogge, R. W. 1988, ApJ, 328, 519

Pogge, R. W., \& De Robertis, M. M. 1995, ApJ, 451, 585

Pogge, R. W., \& Martini, P. 2002, ApJ, 569, 624

Pounds, K. A., Done, C., \& Osborne, J. P. 1995, MNRAS, 277, L5 
Quillen, A. C., Alonso-Herrero, A., Rieke, M. J., McDonald, C., Falcke, H., \& Rieke, G. H. 1999, ApJ, 525, 685

Ramos Almeida, C., Pérez García, A. M., Acosta-Pulido, J. A., Rodríguez Espinosa, J. M., Barrena, R., \& Manchado, A. 2006, ApJ, 645, 148

Rodríguez-Ardila, A., Prieto, M. A., Viegas, S., \& Gruenwald, R. 2006, ApJ, 653, 1098

Ruiz, J. R., Crenshaw, D. M., Kraemer, S. B., Bower, G. A., Gull, T. R., Hutchings, J. B., Kaiser, M. E., \& Weistrop, D. 2001, AJ, 122, 2961

- 2005, AJ, 129, 73

Ruiz, M., Alexander, D. M., Young, S., Hough, J., Lumsden, S. L., \& Heisler, C. A. 2000, MNRAS, 316, 49

Sargent, W. L. W. 1972, ApJ, 173, 7

Schlesinger, K., Pogge, R. W., Martini, P., Shields, J. C., \& Fields, D. 2009, ApJ, 699, 857

Schmitt, H. R., Antonucci, R. R. J., Ulvestad, J. S., Kinney, A. L., Clarke, C. J., \& Pringle, J. E. 2001, ApJ, 555, 663

Schmitt, H. R., Donley, J. L., Antonucci, R. R. J., Hutchings, J. B., \& Kinney, A. L. 2003, ApJS, 148, 327

Schmitt, H. R., \& Kinney, A. L. 1996, ApJ, 463, 498

—. 2000, ApJS, 128, 479

Schmitt, H. R., Storchi-Bergmann, T., \& Baldwin, J. A. 1994, ApJ, 423, 237

Seyfert, C. K. 1943, ApJ, 97, 28

Shu, X. W., Wang, J. X., Jiang, P., Fan, L. L., \& Wang, T. G. 2007, ApJ, 657, 167

Simpson, C., Wilson, A. S., Bower, G., Heckman, T. M., Krolik, J. H., \& Miley, G. K. 1997, ApJ, 474, 121

Sparke, L. S., \& Gallagher, III, J. S. 2000, Galaxies in the universe : an introduction (Cambridge University Press)

Springob, C. M., Haynes, M. P., Giovanelli, R., \& Kent, B. R. 2005, ApJS, 160, 149 
Storchi-Bergmann, T., Lopes, R. D. S., McGregor, P. J., Riffel, R. A., Beck, T., \& Martini, P. 2010, MNRAS, 402, 819

Storchi-Bergmann, T., Wilson, A. S., \& Baldwin, J. A. 1992, ApJ, 396, 45

Storchi-Bergmann, T., Wilson, A. S., Mulchaey, J. S., \& Binette, L. 1996, A\&A, 312, 357

Toomre, A. 1981, in Structure and Evolution of Normal Galaxies, ed. S. M. Fall \& D. LyndenBell (Cambridge University Press), 111

Tremaine, S. et al. 2002, ApJ, 574, 740

Trippe, M. L., Crenshaw, D. M., Deo, R. P., Dietrich, M., Kraemer, S. B., Rafter, S. E., \& Turner, T. J. 2010, ApJ, 725, 1749

Tsvetanov, Z., \& Walsh, J. R. 1992, ApJ, 386, 485

Turner, T. J., \& Miller, L. 2009, A\&A Rev., 17, 47

Ulvestad, J. S., Antonucci, R. R. J., \& Goodrich, R. W. 1995, AJ, 109, 81

Ulvestad, J. S., \& Wilson, A. S. 1984, ApJ, 285, 439

Unger, S. W., Pedlar, A., Axon, D. J., Whittle, M., Meurs, E. J. A., \& Ward, M. J. 1987, MNRAS, 228, 671

Vestergaard, M., \& Peterson, B. M. 2006, ApJ, 641, 689

Vrtilek, J. M., \& Carleton, N. P. 1985, ApJ, 294, 106

Walsh, J. L., Barth, A. J., Ho, L. C., Filippenko, A. V., Rix, H.-W., Shields, J. C., Sarzi, M., \& Sargent, W. L. W. 2008, AJ, 136, 1677

Weymann, R. J., Morris, S. L., Foltz, C. B., \& Hewett, P. C. 1991, ApJ, 373, 23

Whittle, M. 1985, MNRAS, 213, 1

Whittle, M., Pedlar, A., Meurs, E. J. A., Unger, S. W., Axon, D. J., \& Ward, M. J. 1988, ApJ, 326, 125

Whittle, M., Rosario, D. J., Silverman, J. D., Nelson, C. H., \& Wilson, A. S. 2005, AJ, 129, 104

Whittle, M., \& Wilson, A. S. 2004, AJ, 127, 606 
Whittle, M., Wilson, A. S., Nelson, C. H., Rosario, D., \& Silverman, J. D. 2002, in Revista Mexicana de Astronomia y Astrofisica, vol. 27, Vol. 13, Revista Mexicana de Astronomia y Astrofisica Conference Series, ed. W. J. Henney, W. Steffen, L. Binette, \& A. Raga, $230-235$

Wilson, A. S., Shopbell, P. L., Simpson, C., Storchi-Bergmann, T., Barbosa, F. K. B., \& Ward, M. J. 2000, AJ, 120, 1325

Wilson, A. S., \& Tsvetanov, Z. I. 1994, AJ, 107, 1227

Winter, L. M., Mushotzky, R. F., Reynolds, C. S., \& Tueller, J. 2009, ApJ, 690, 1322

Woo, J.-H., \& Urry, C. M. 2002, ApJ, 579, 530

Xilouris, E. M., Byun, Y. I., Kylafis, N. D., Paleologou, E. V., \& Papamastorakis, J. 1999, A\&A, 344, 868

Xilouris, E. M., \& Papadakis, I. E. 2002, A\&A, 387, 441

Yan, R., \& Blanton, M. R. 2012, ApJ, 747, 61 


\section{APPENDIX A}

\section{Extended Sample: HST STIS Slit Positions}

The appendix contains HST images of the extended sample. Filters centered on [O III] were used if available, otherwise F606W or F547M continuum filters are shown. Plus signs give the location of the optical continuum peaks. Solid lines outline the position of each STIS slit. Details for each observation are listed in Table 6.2. Images without slits are for targets using slitless STIS observations.

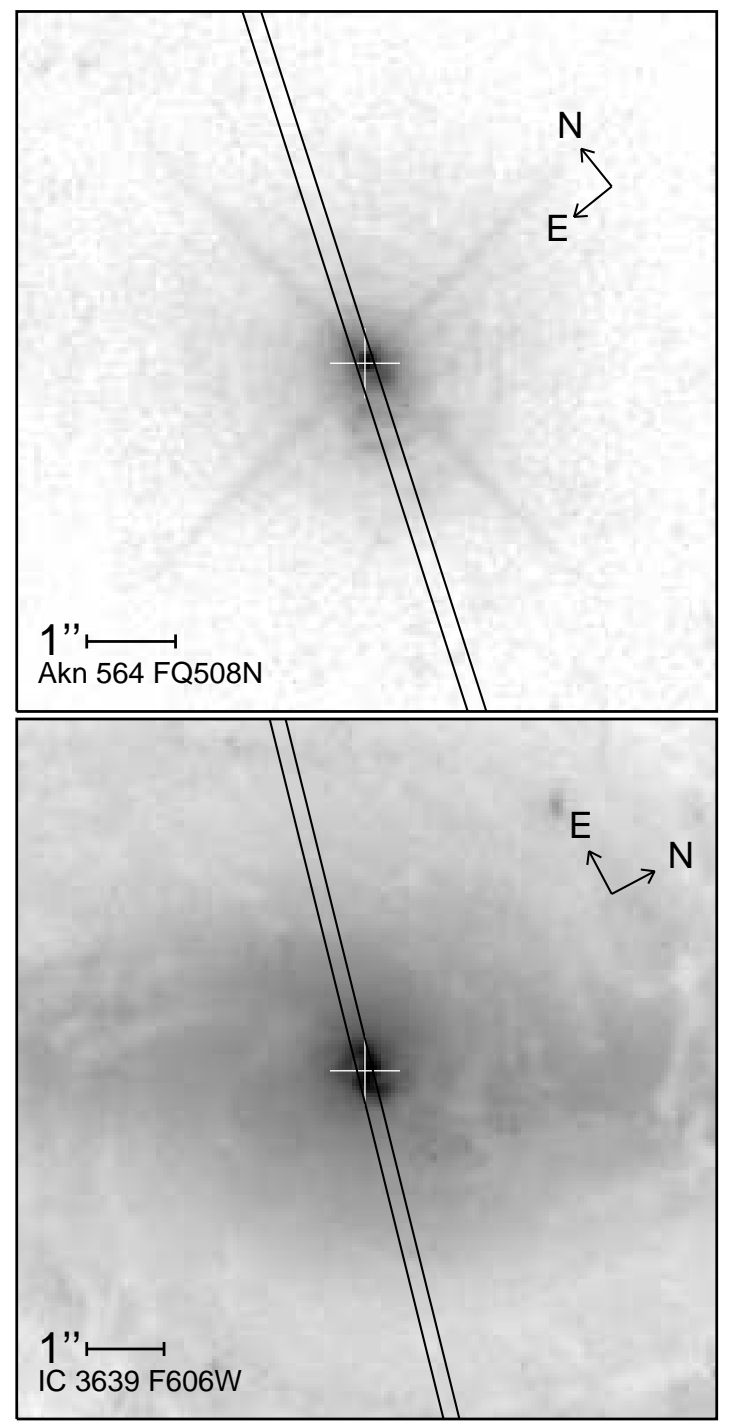

Figure A.1

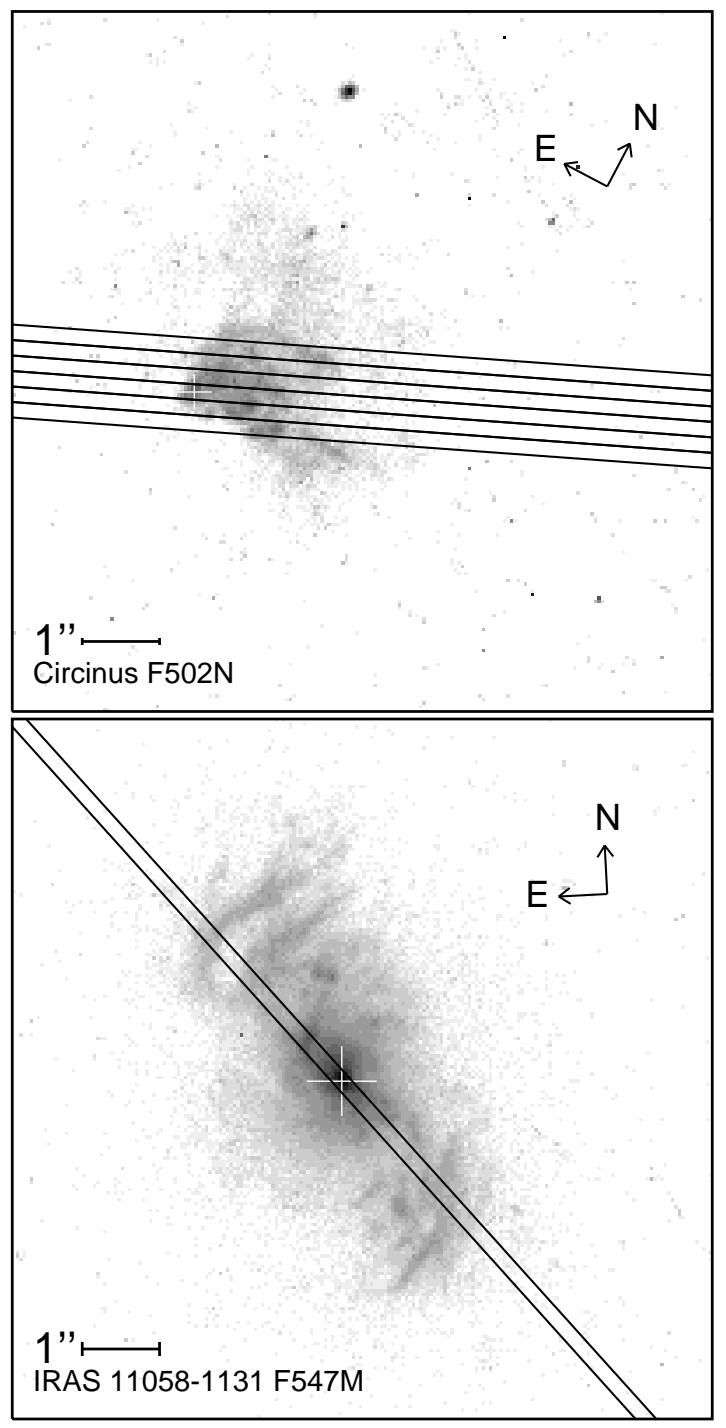



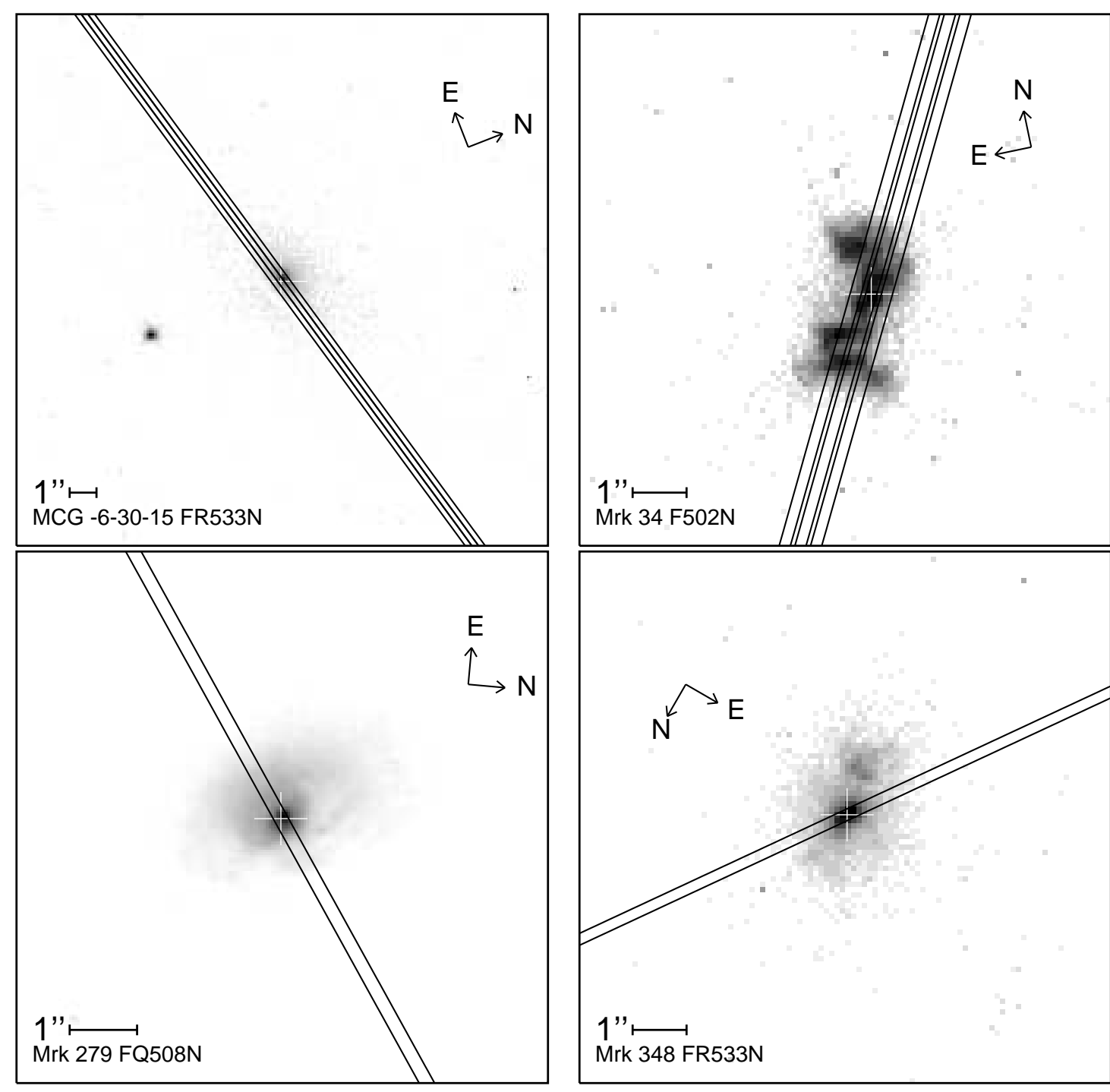

Figure A.2 

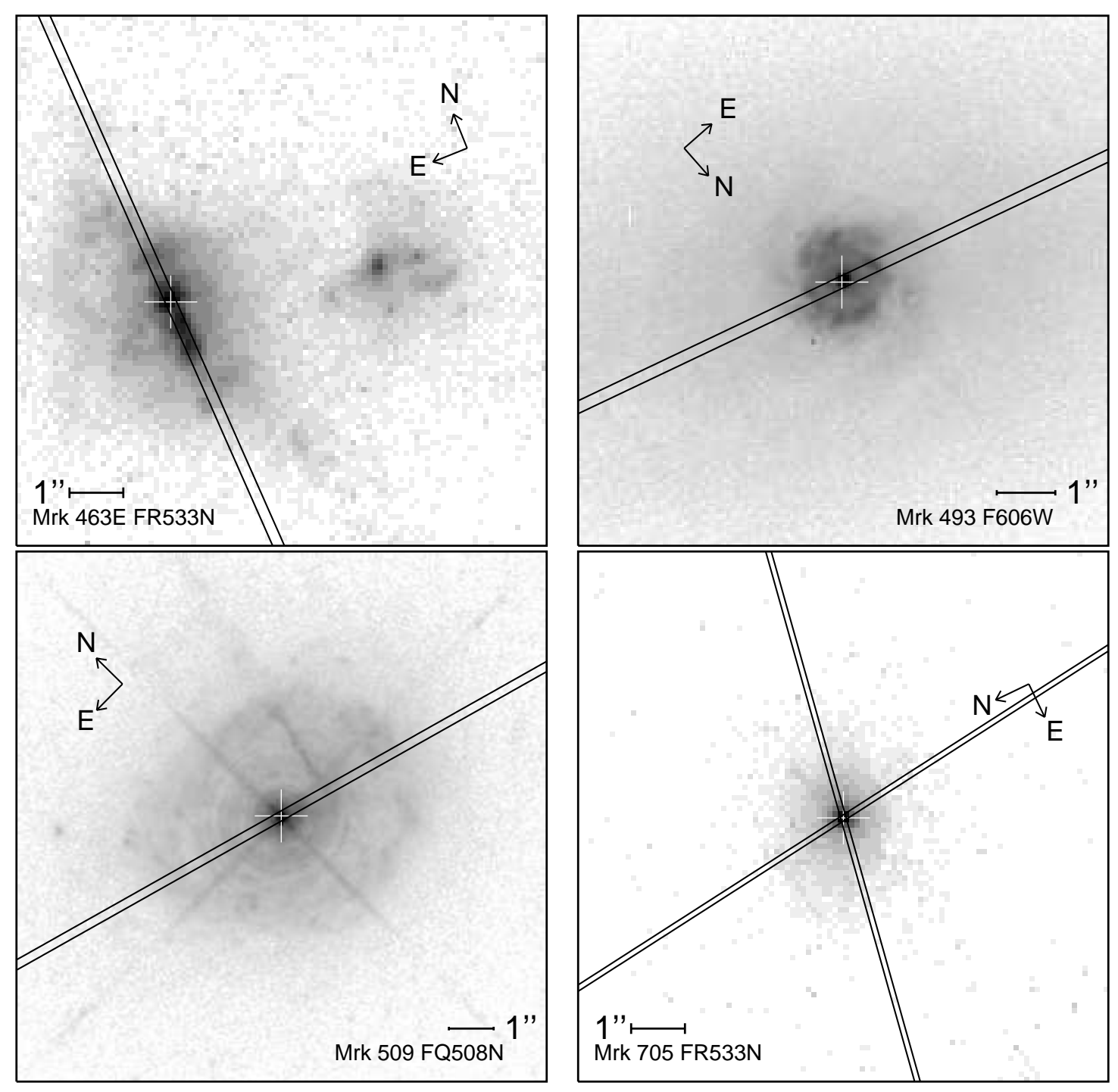

Figure A.3 

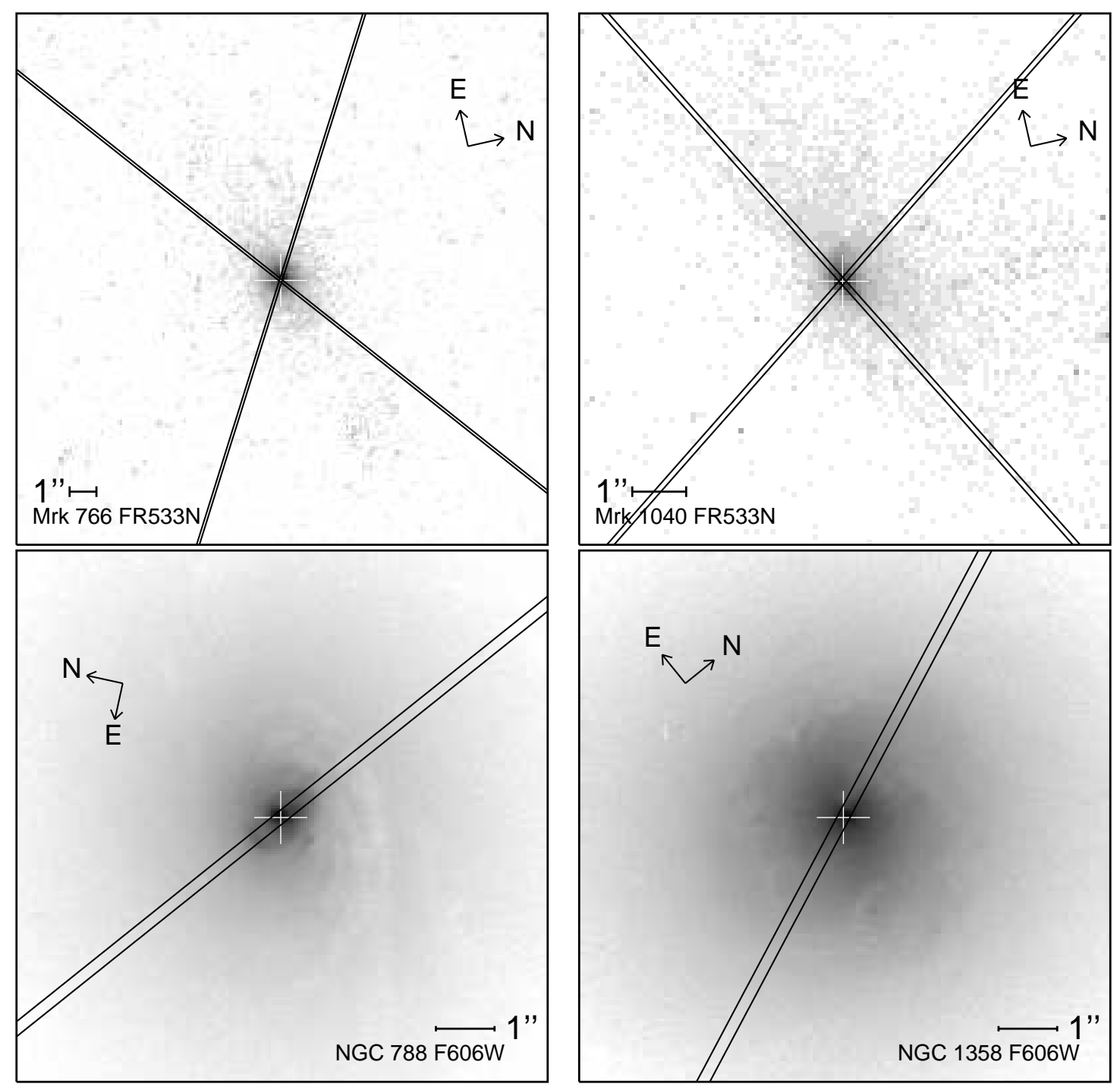

Figure A.4 

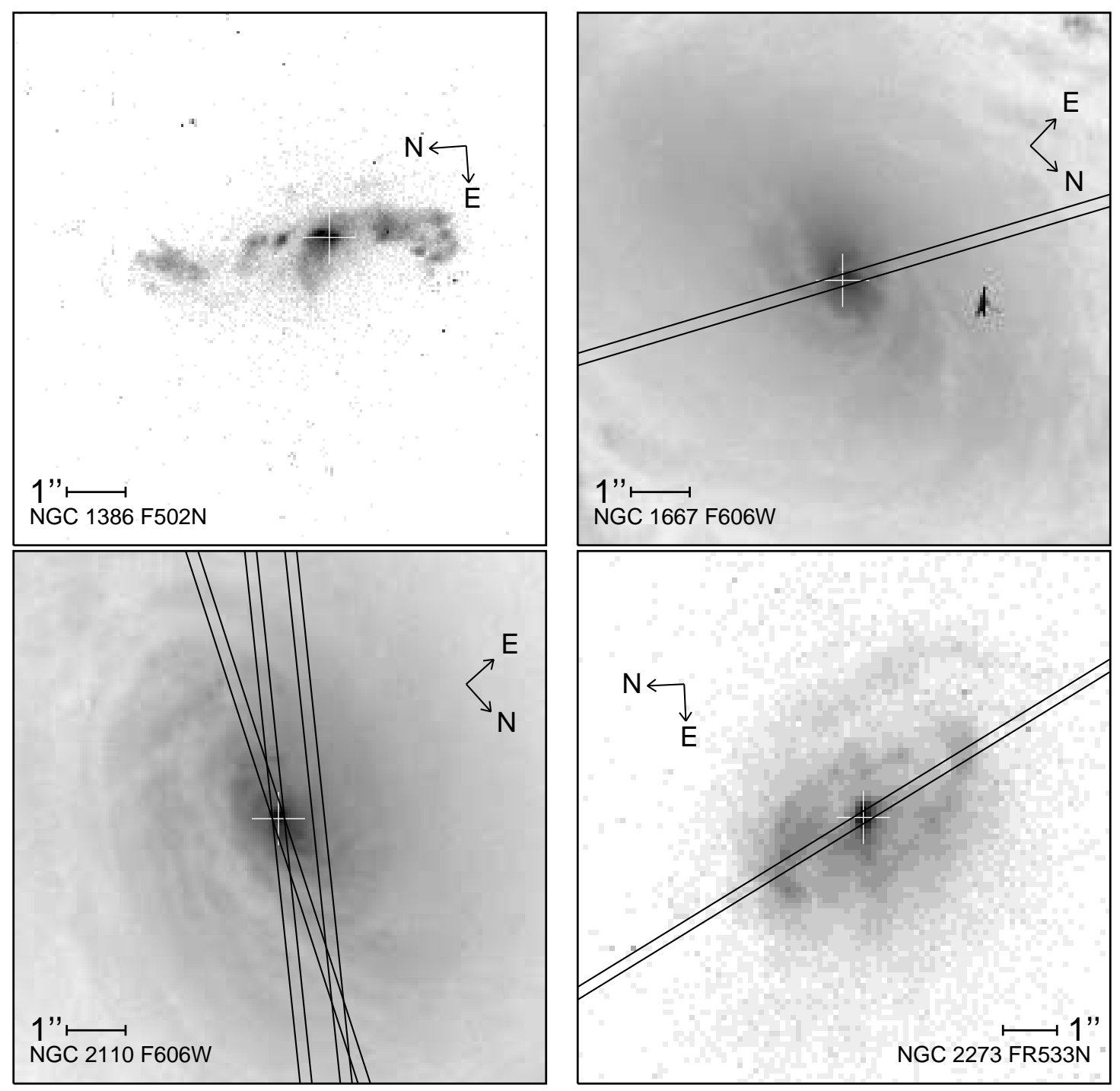

Figure A.5 

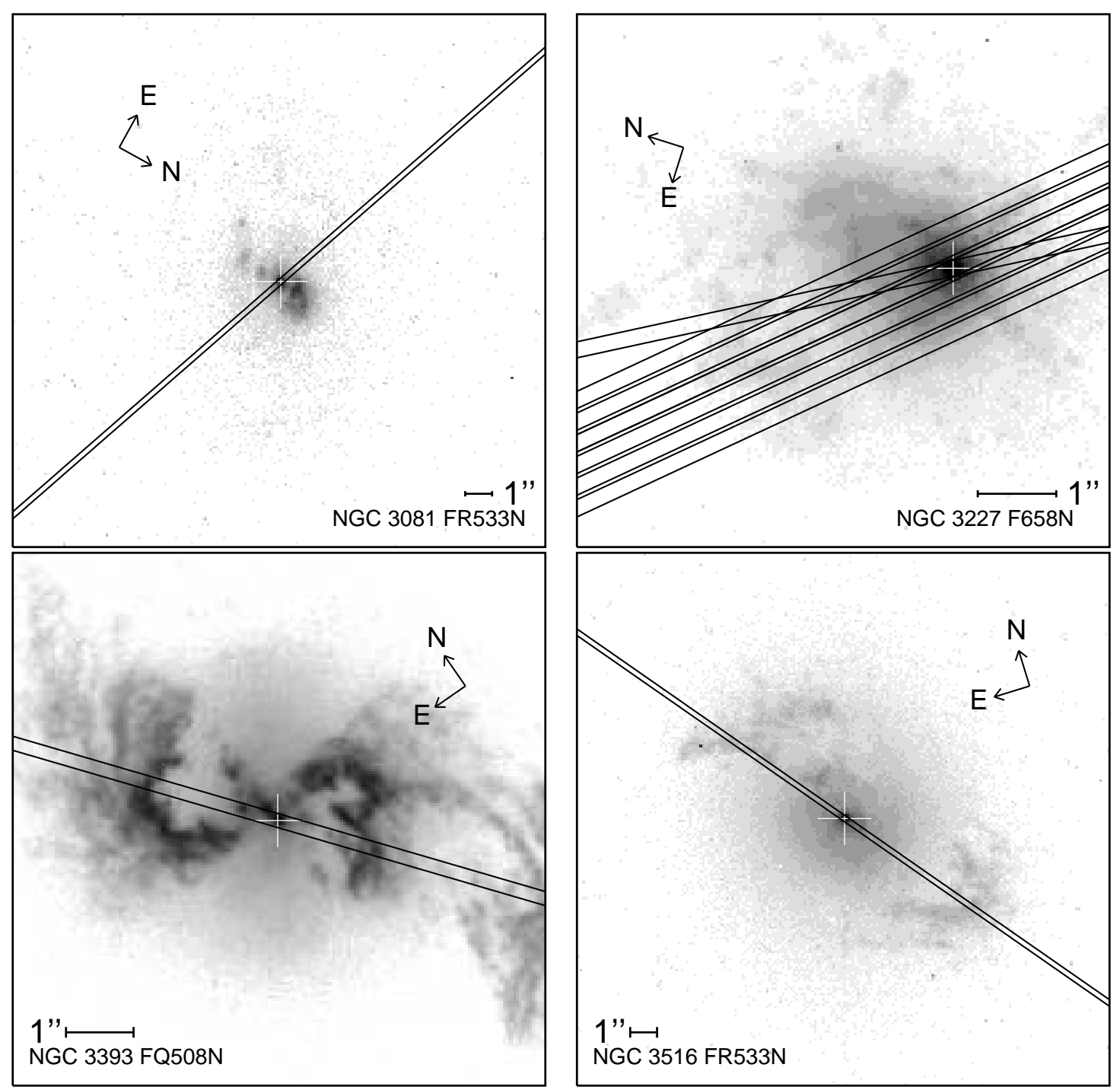

Figure A.6 

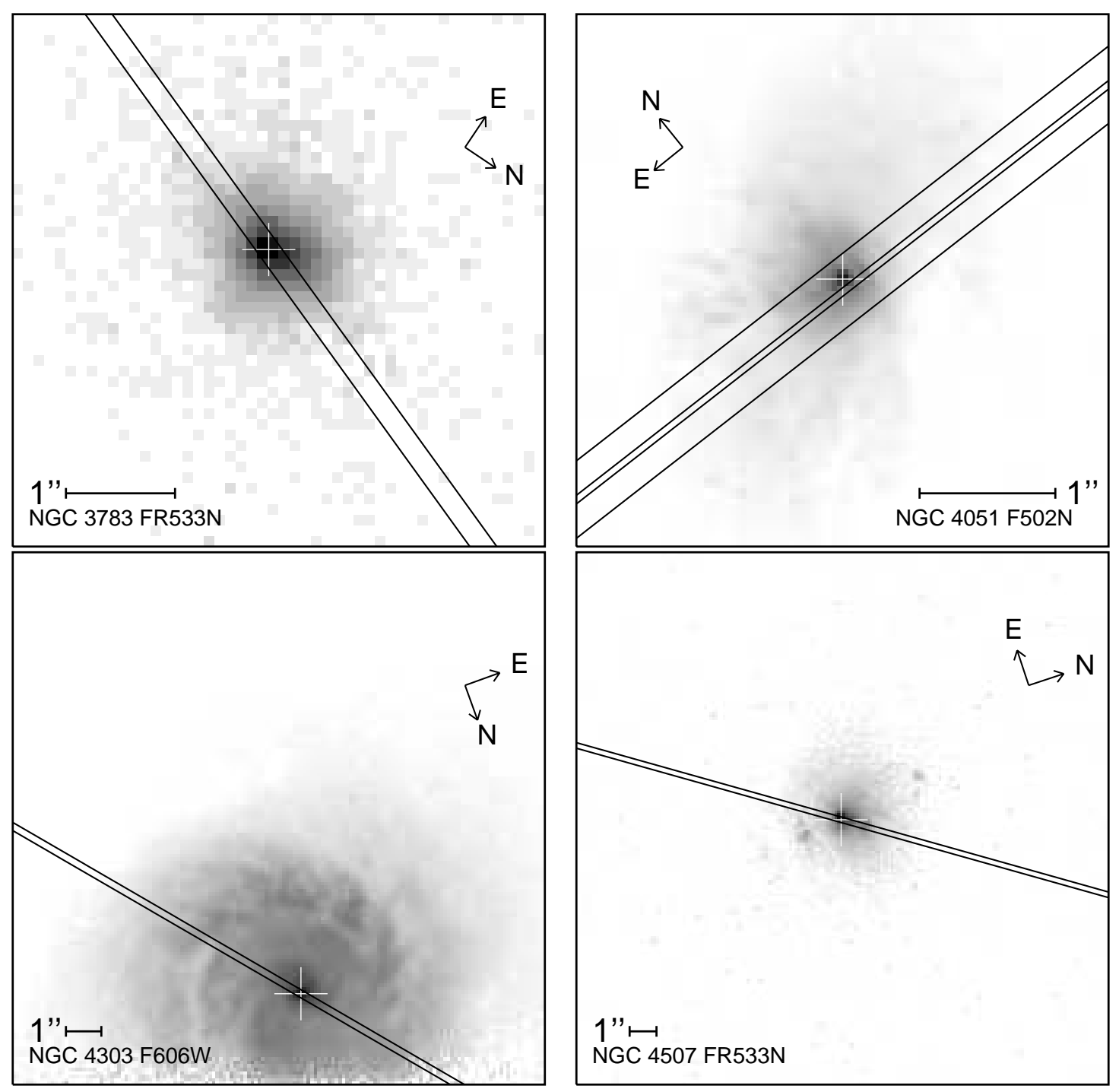

Figure A.7 

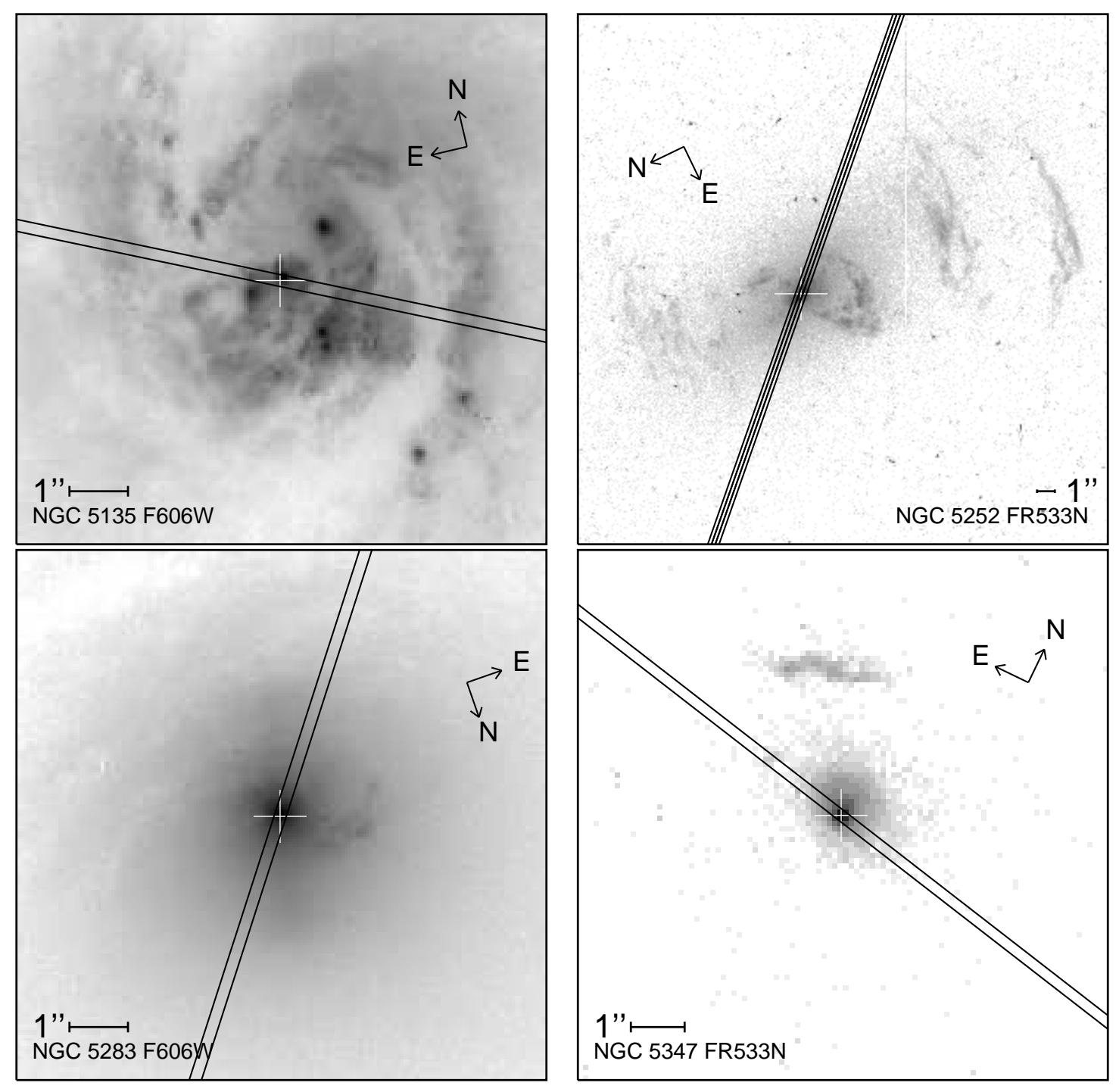

Figure A.8 

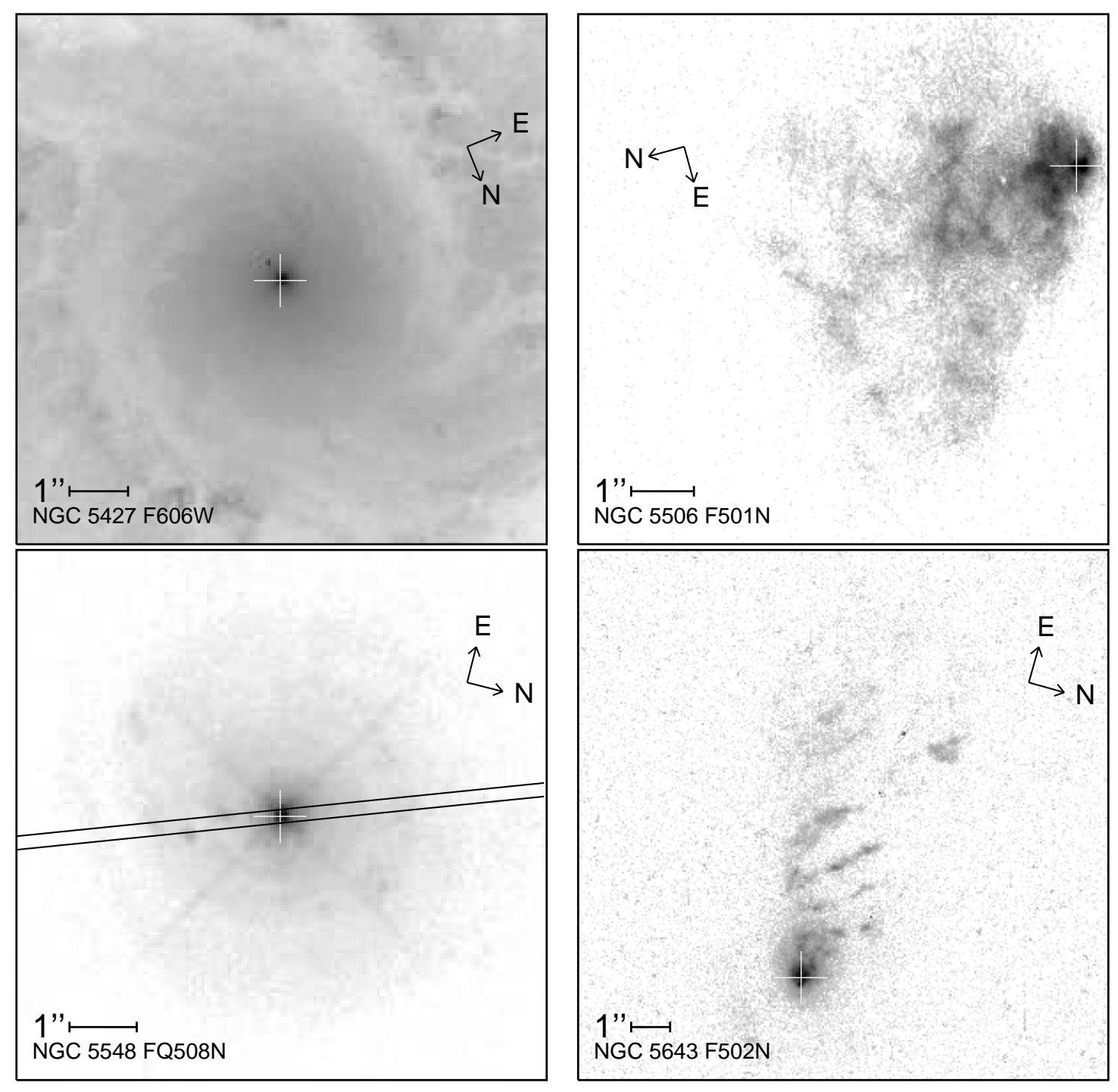

Figure A.9 

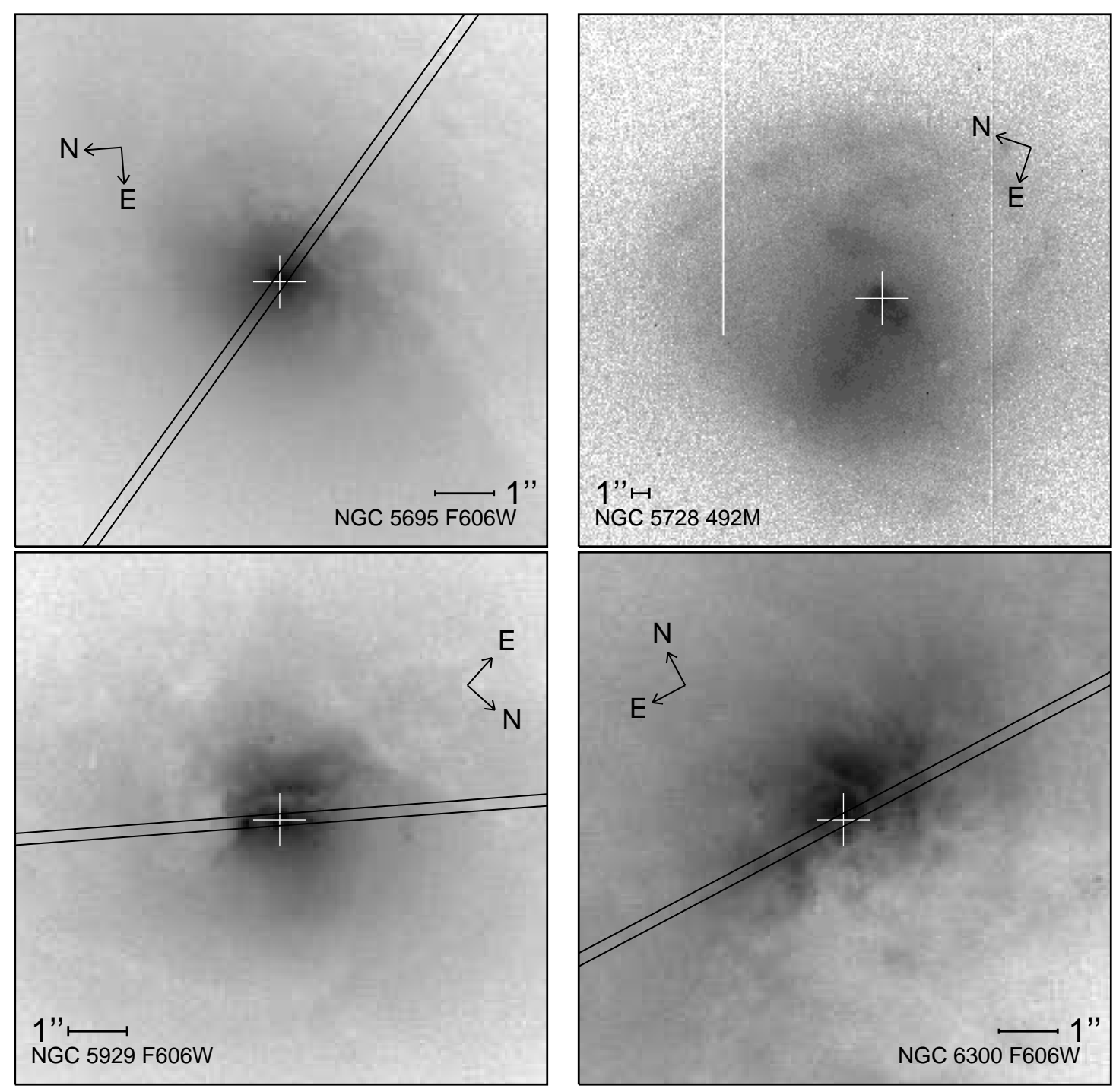

Figure A.10 

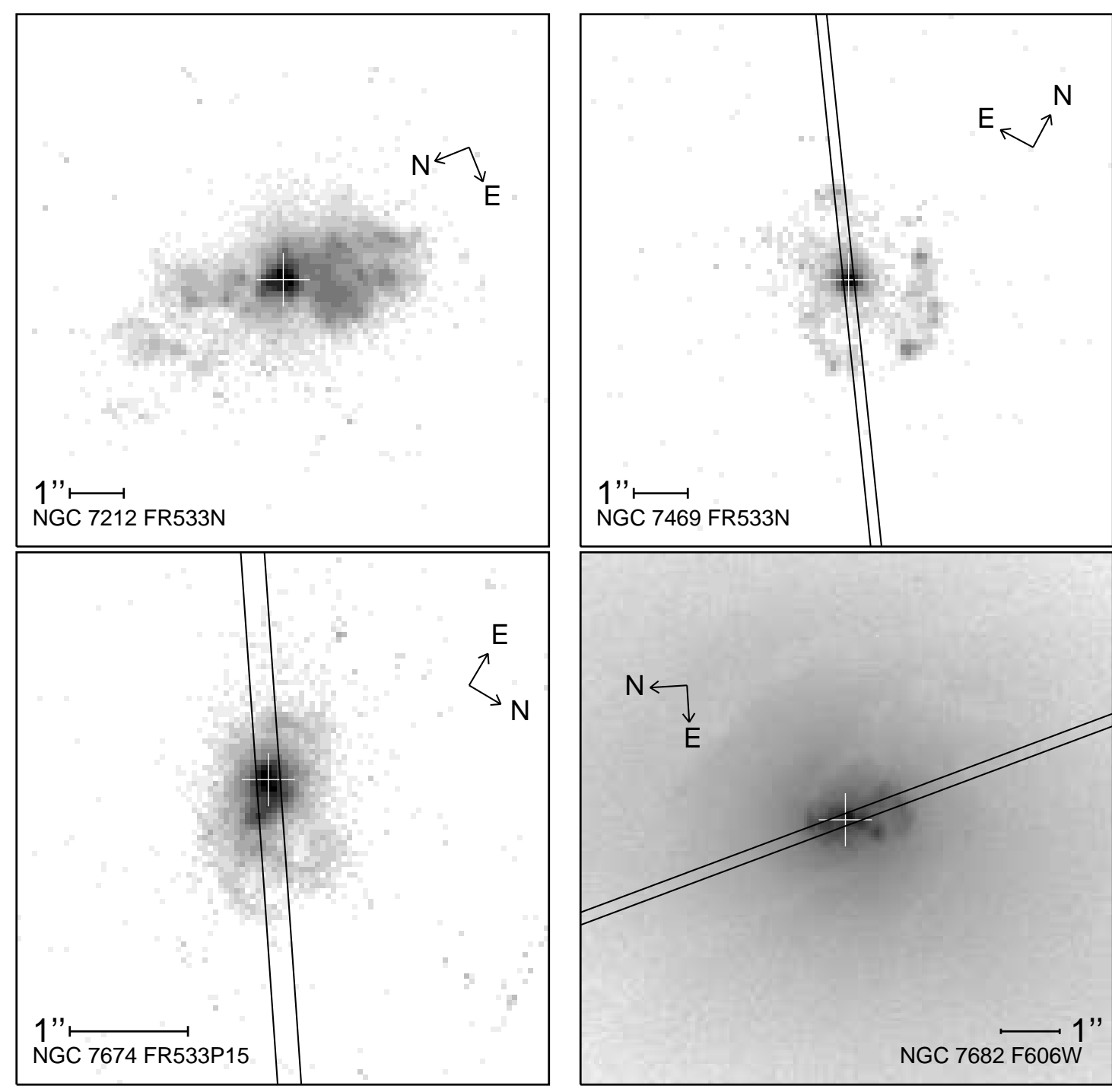

Figure A.11 


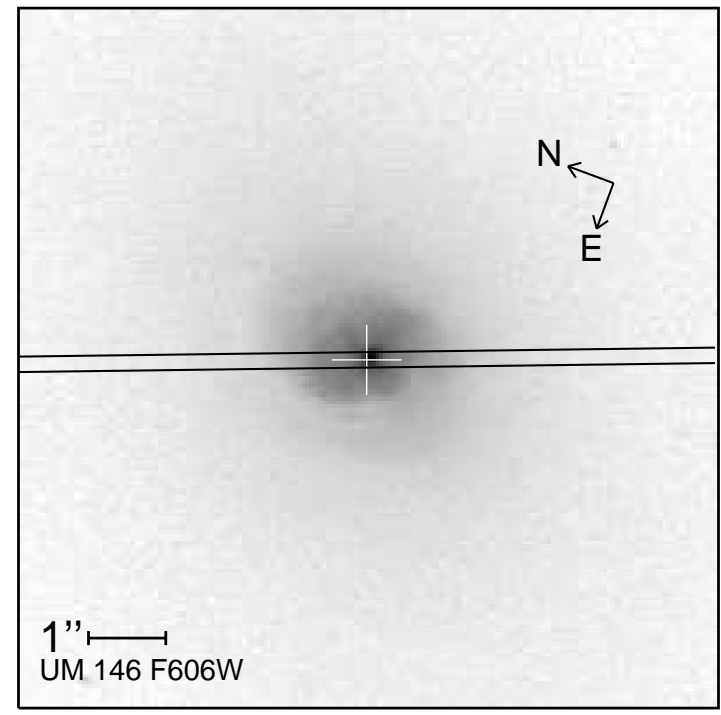

Figure A.12 


\section{APPENDIX B}

\section{Extended Sample: Spectral Images}

This appendix contains HST STIS long-slit spectral images of target sample. Wavelength increases from left to right. G430L images contain $\mathrm{H} \beta \lambda 4861$, [O III] $\lambda 4959$, and [O III] $\lambda 5007$ from left to right respectively. G430M images contain [O III] $\lambda 4959$ and [O III] $\lambda 5007$

to the left and right respectively. G750M images contain [N II] $\lambda 6548, \mathrm{H} \alpha \lambda 6563$, and [N II] $\lambda 6583$ from left to right respectively. The horizontal dashed line gives the location of the continuum centroid. 

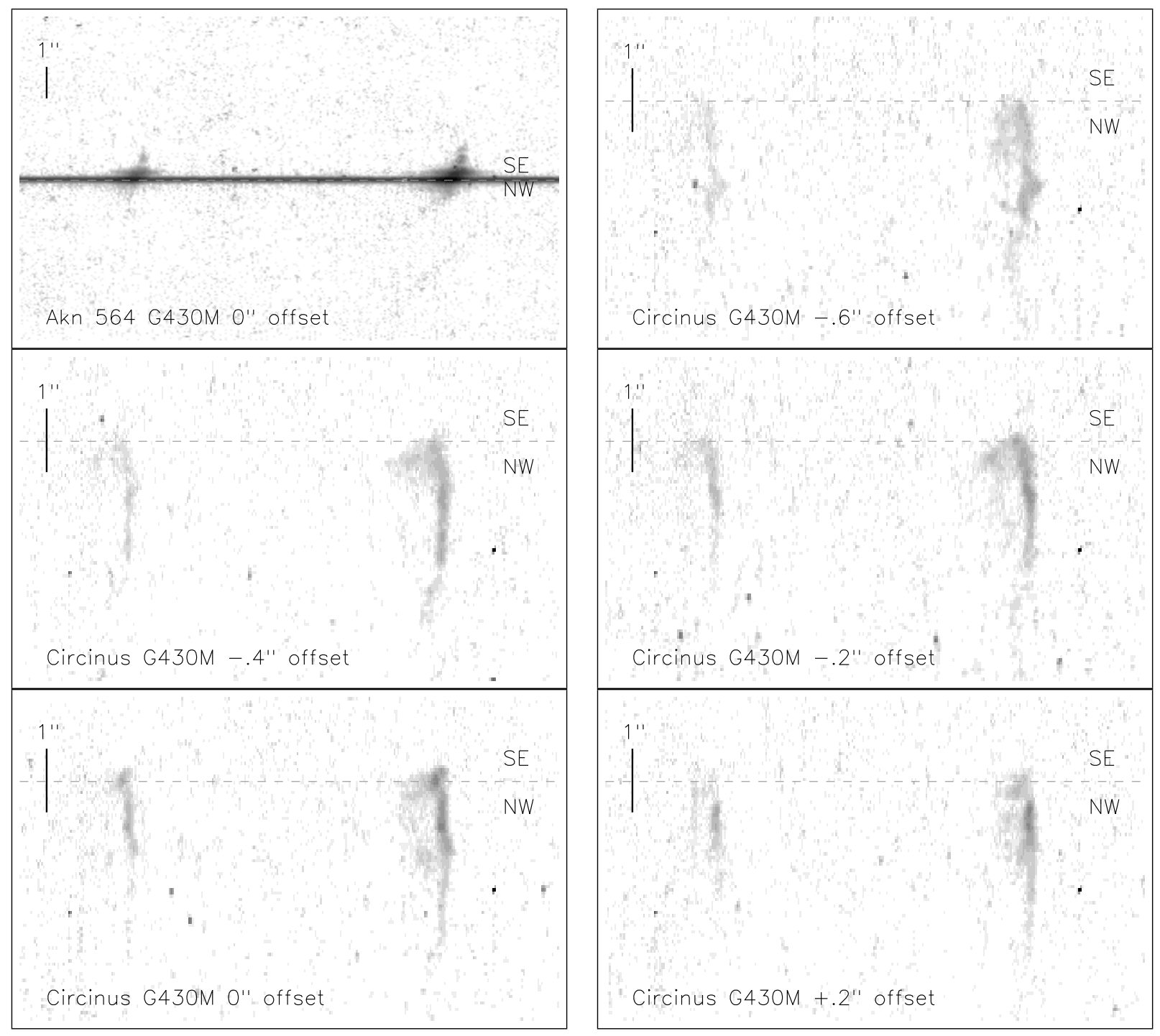

Figure B.1 

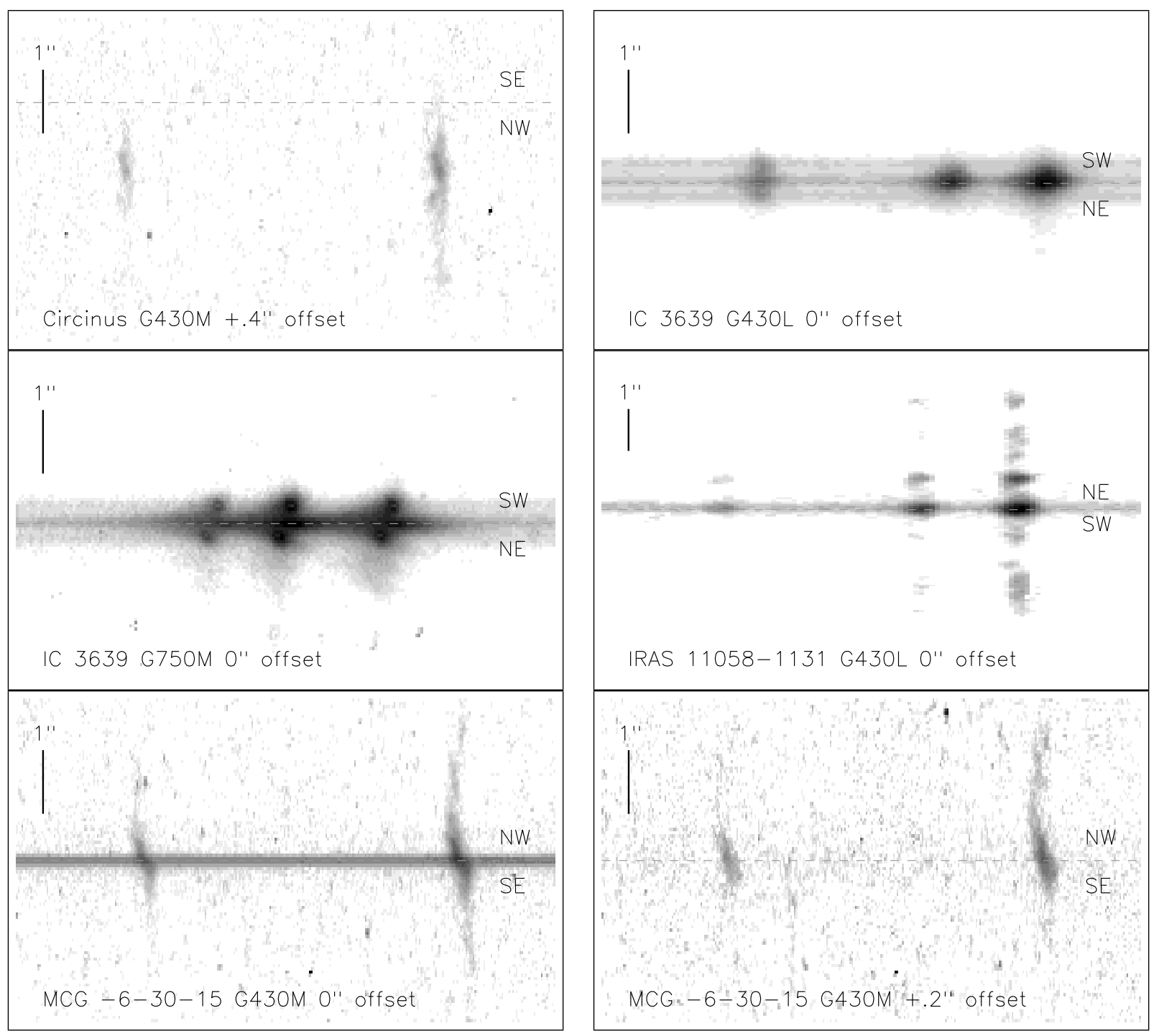

IRAS 11058-1131 G430L O" offset

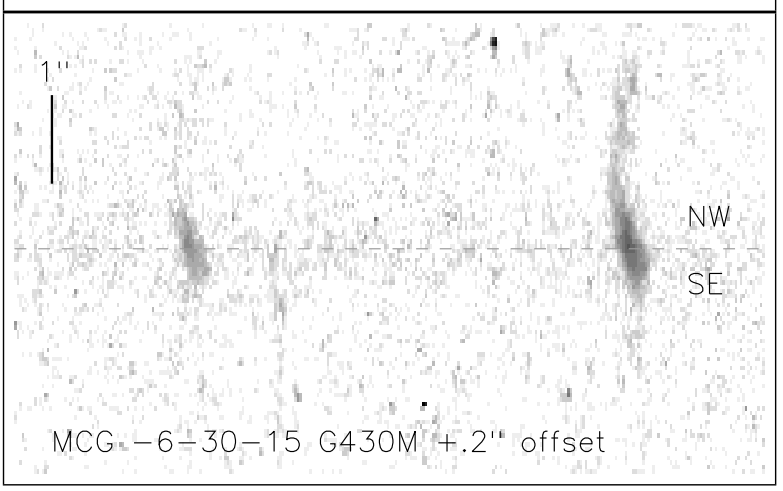

Figure B.2 

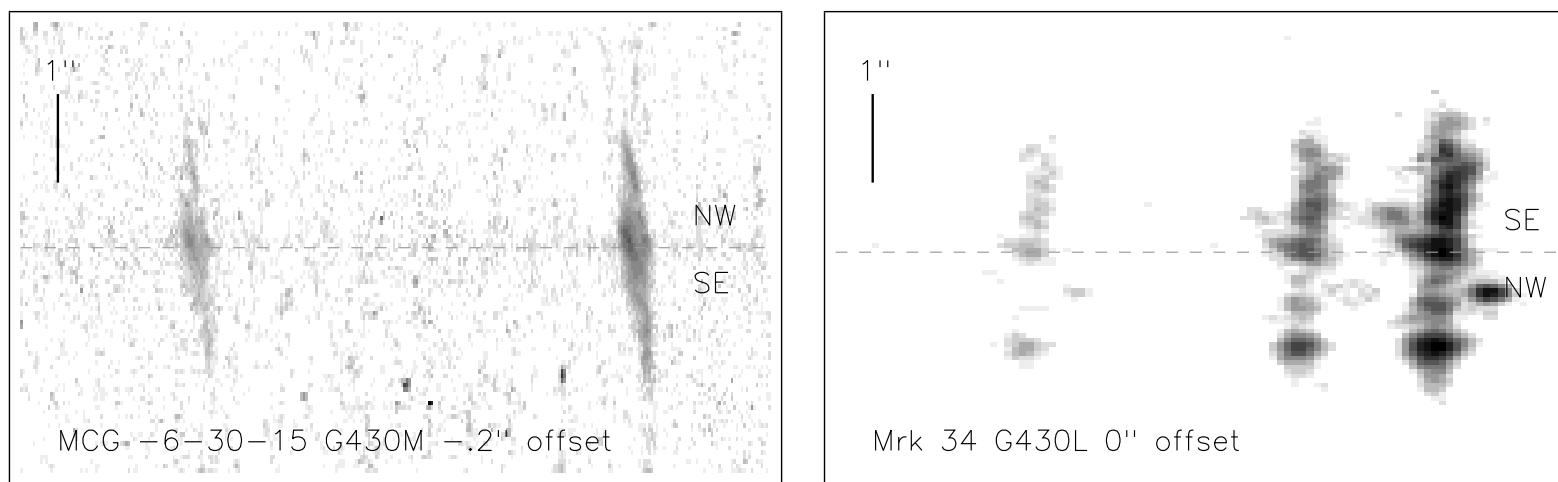

Mrk 34 G430L O" offset
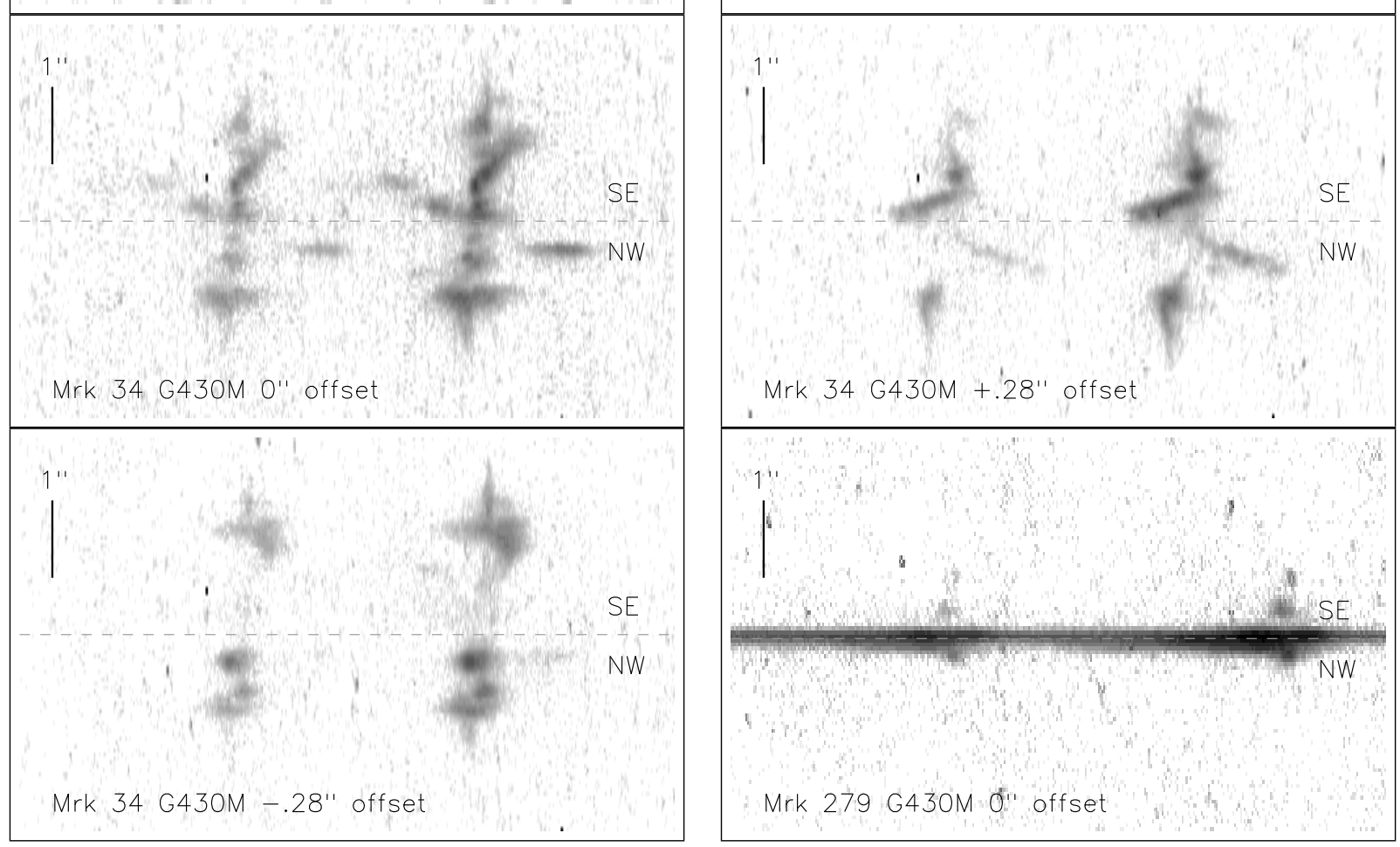

Figure B.3 

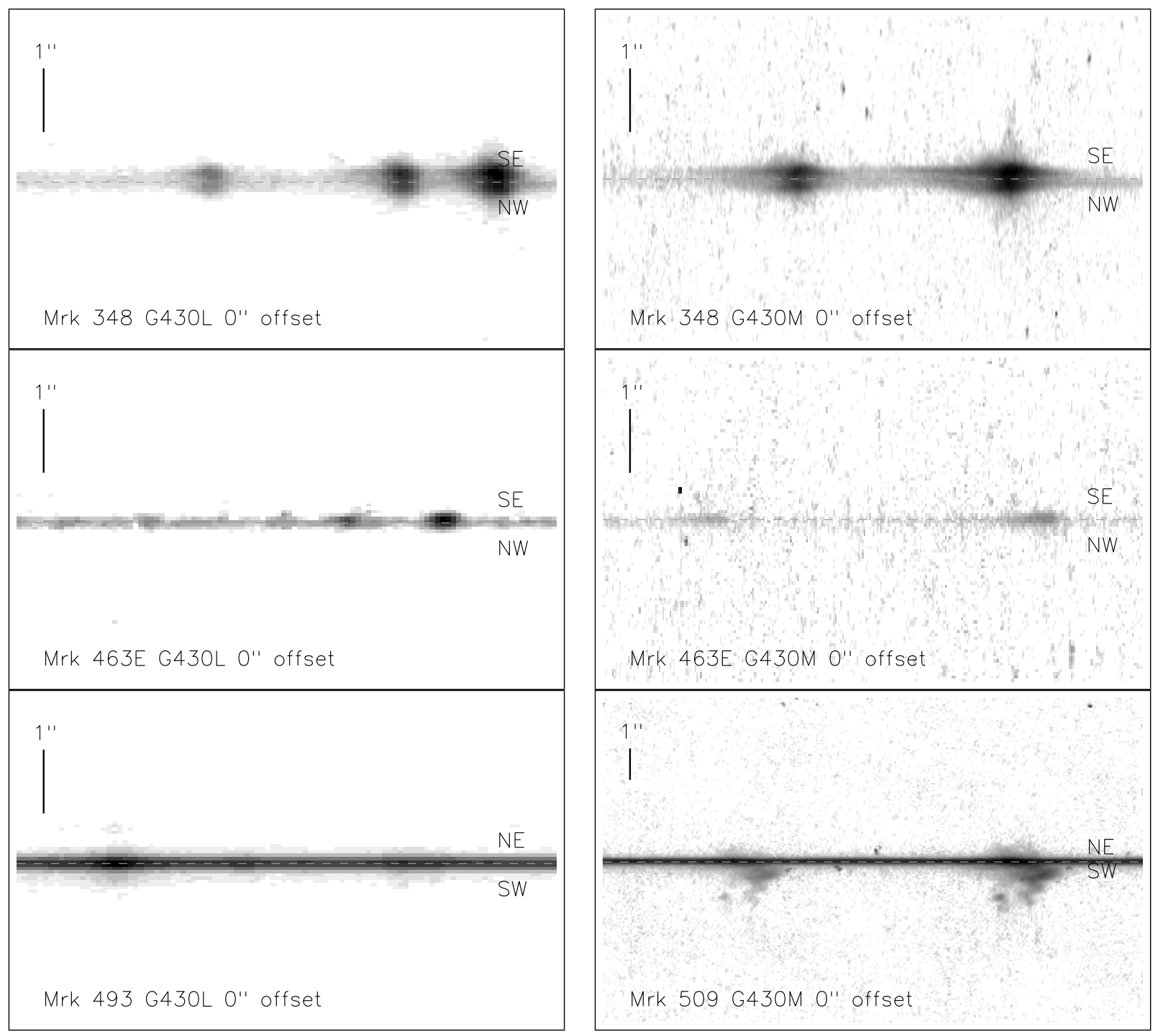

Figure B.4 

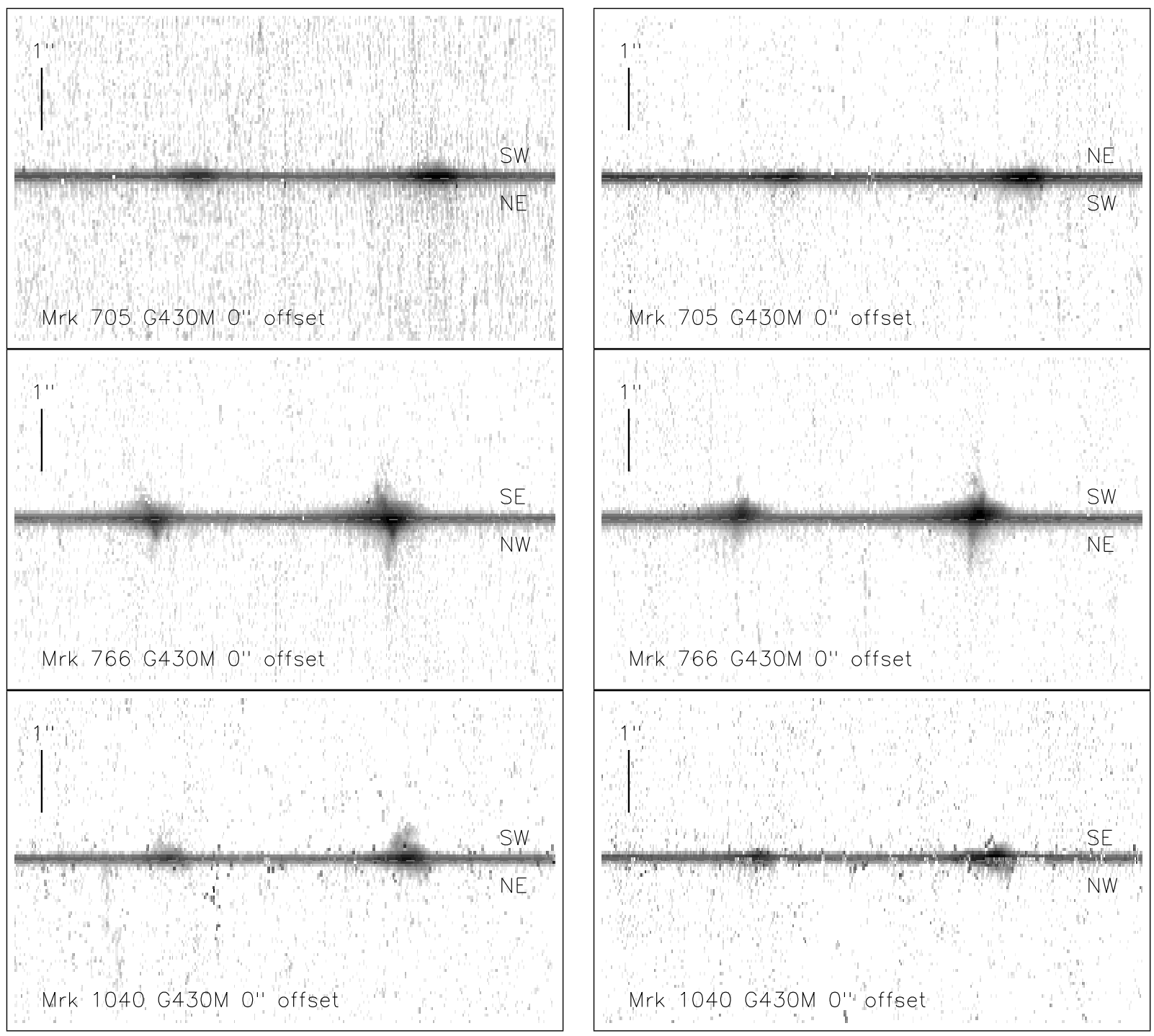

Figure B.5 

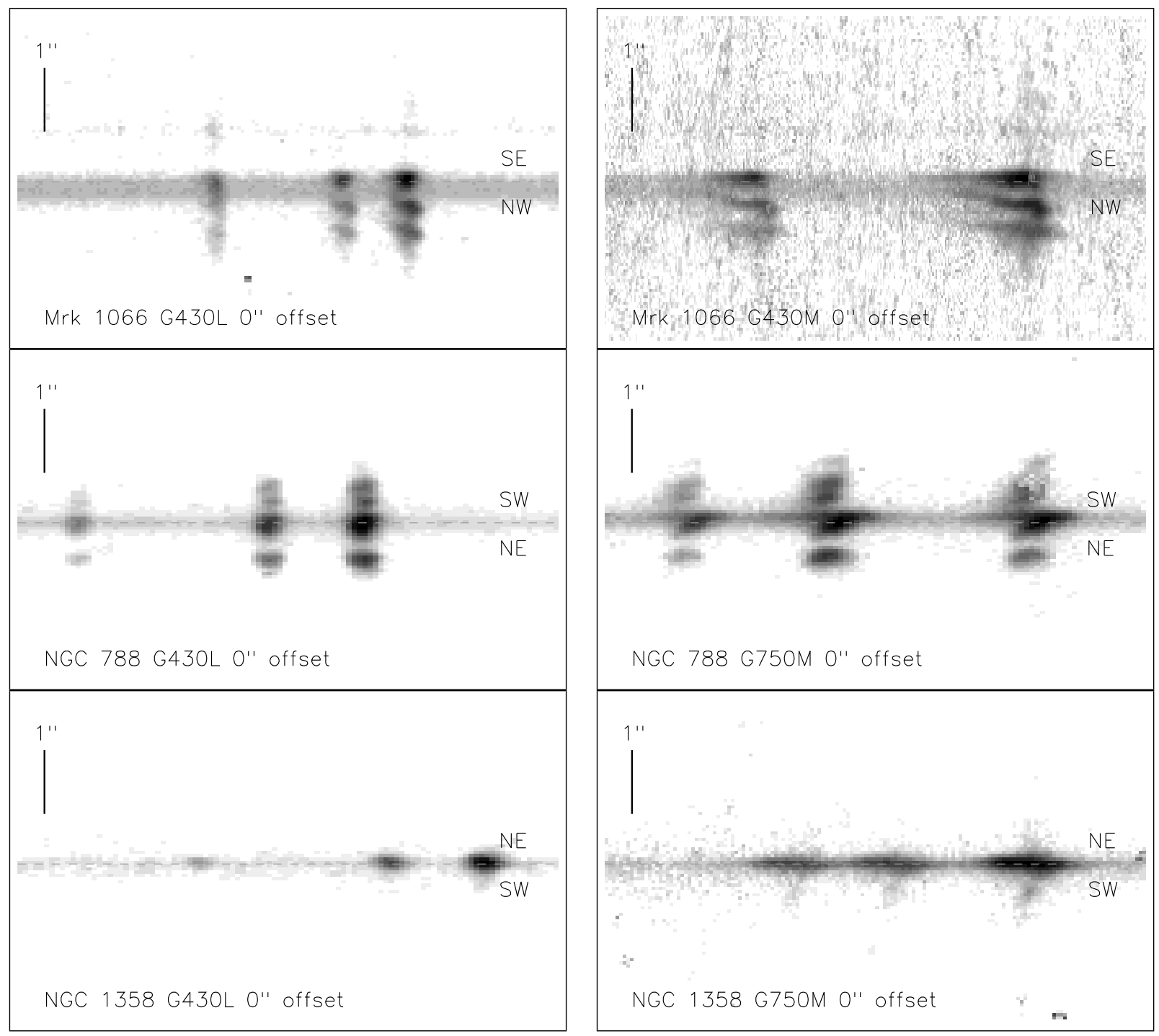

Figure B.6 

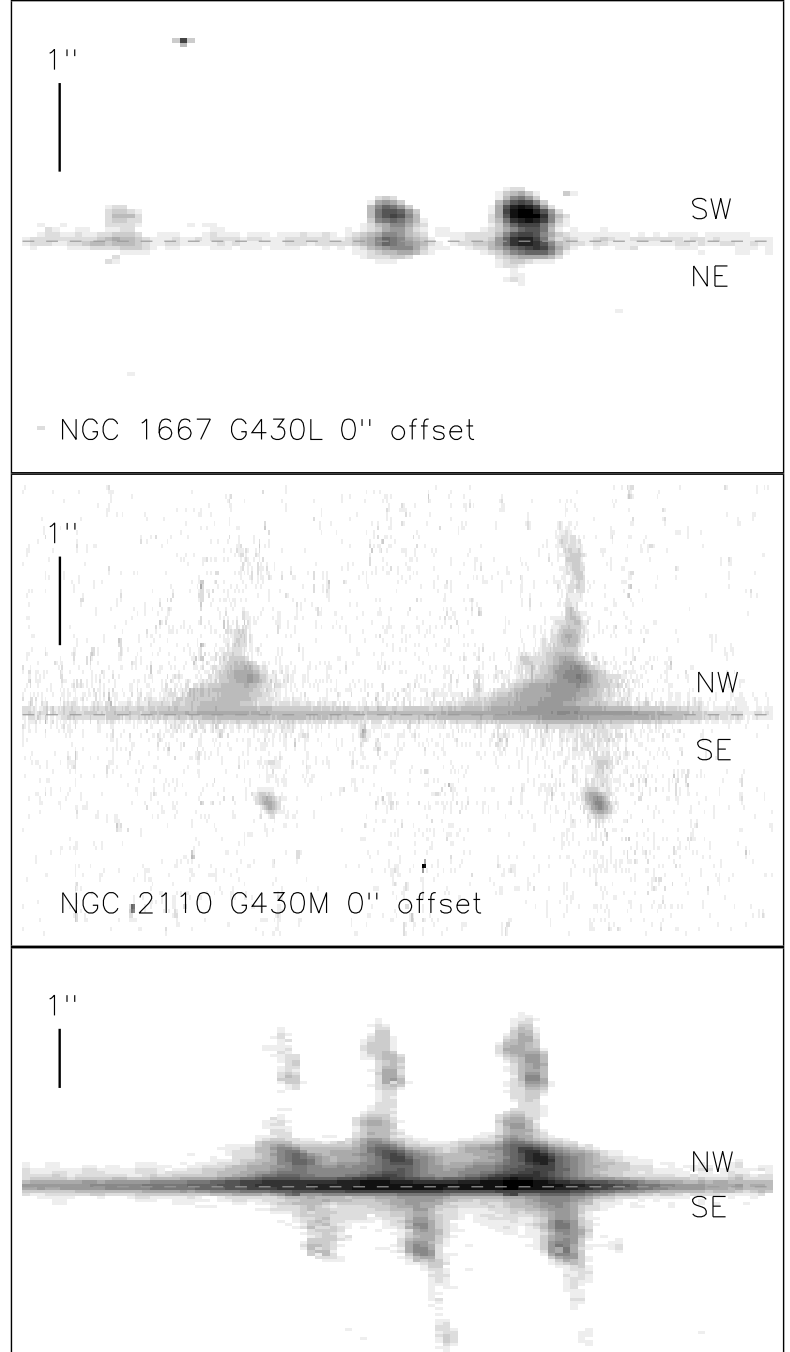

NGC 2110 G750M O" offset

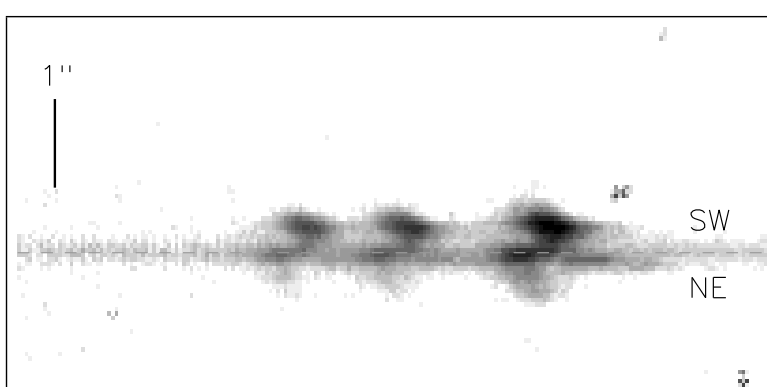

NGC 1667 G750M O" offset

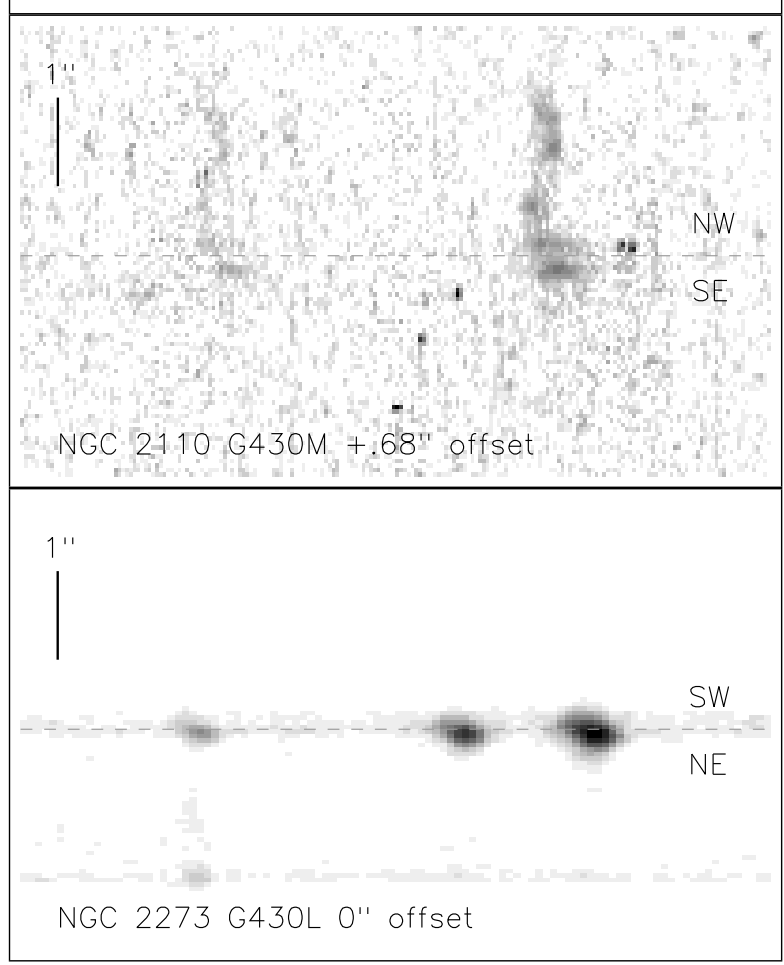

Figure $\quad$ B.7 

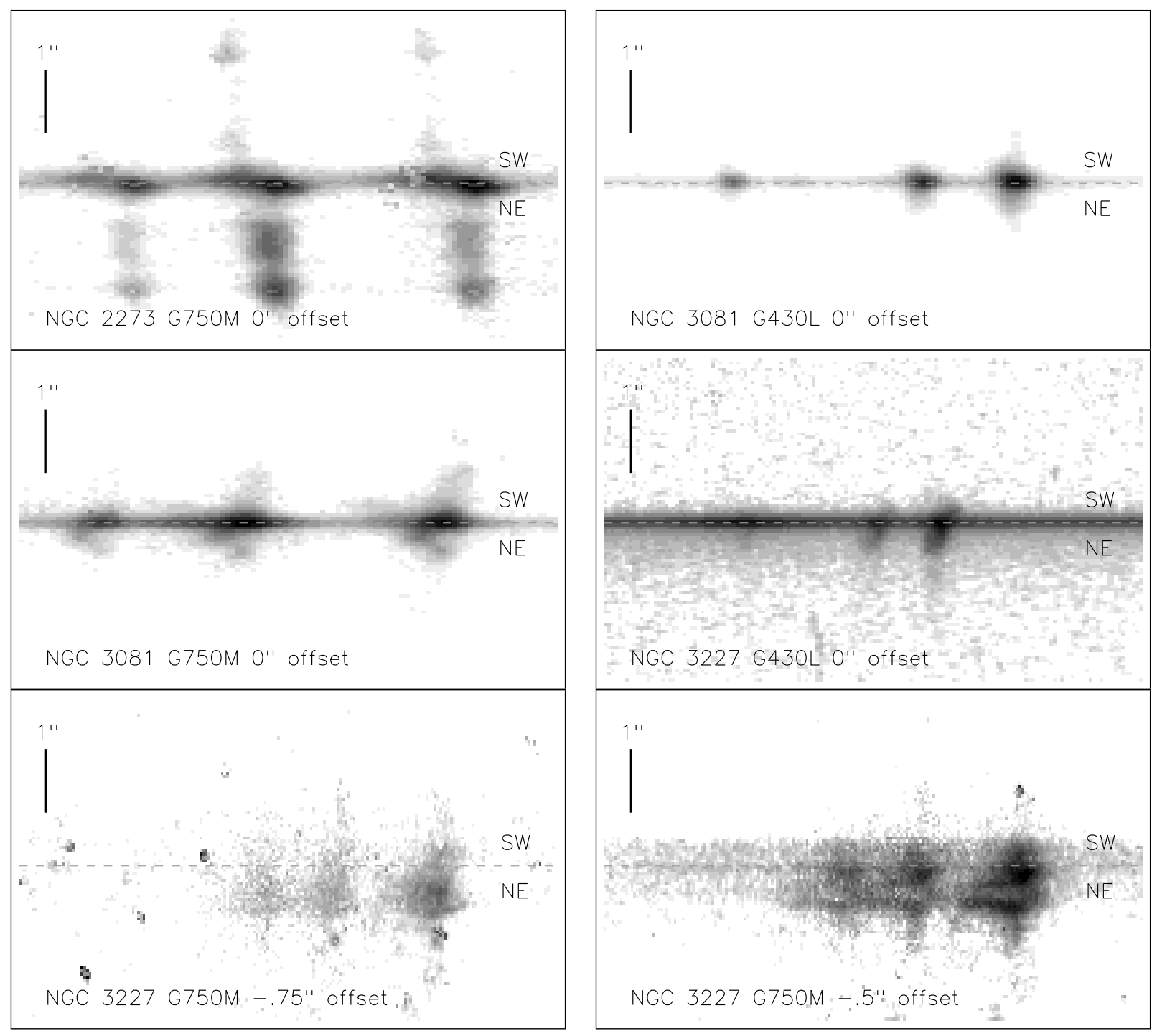

Figure B.8 

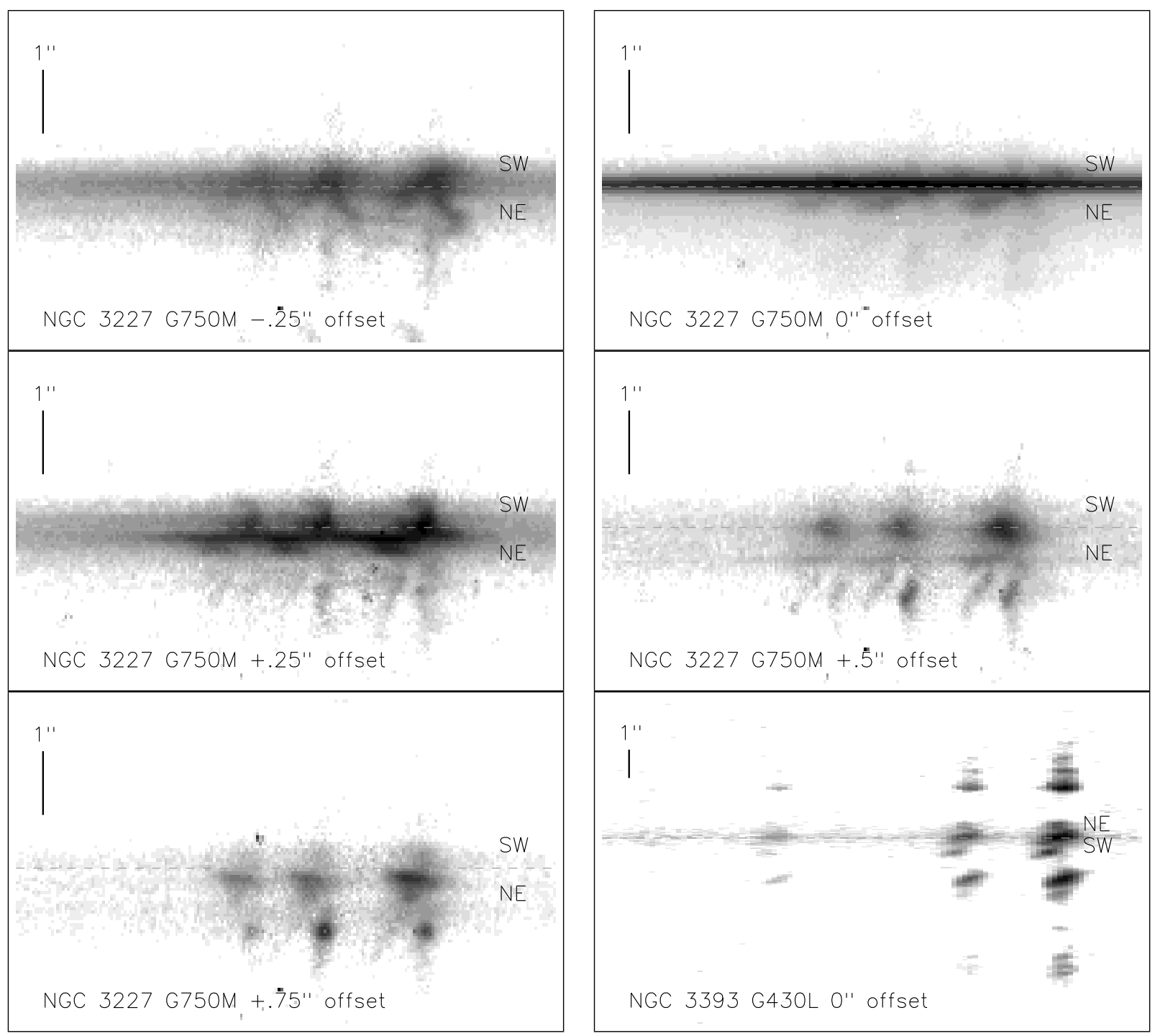

NGC 3227 G750M 0" offset

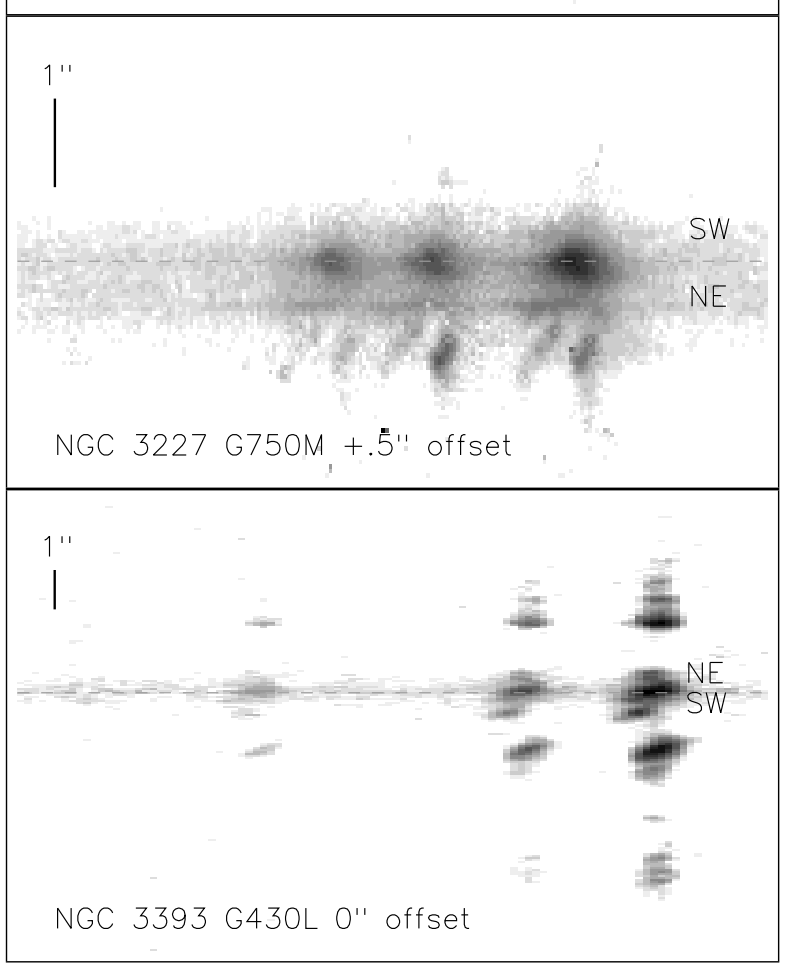

Figure B.9 


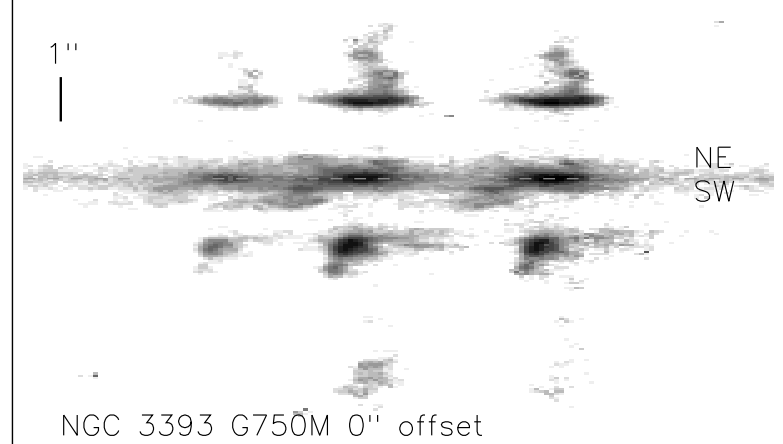

NGC 3393 G750M O" offset

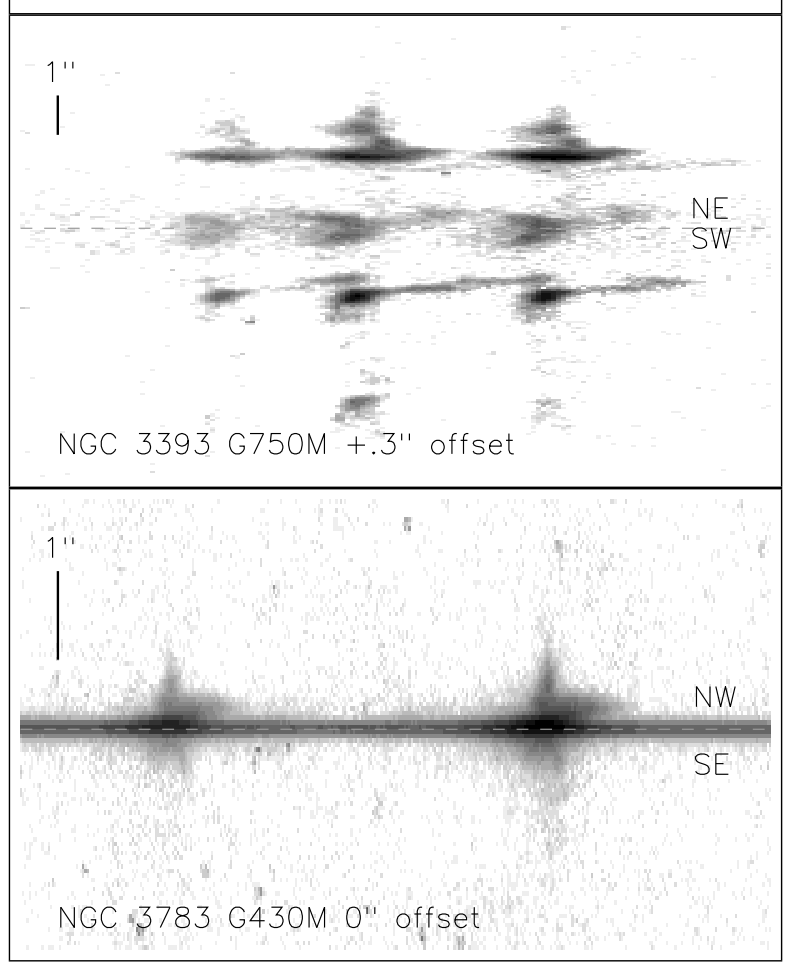

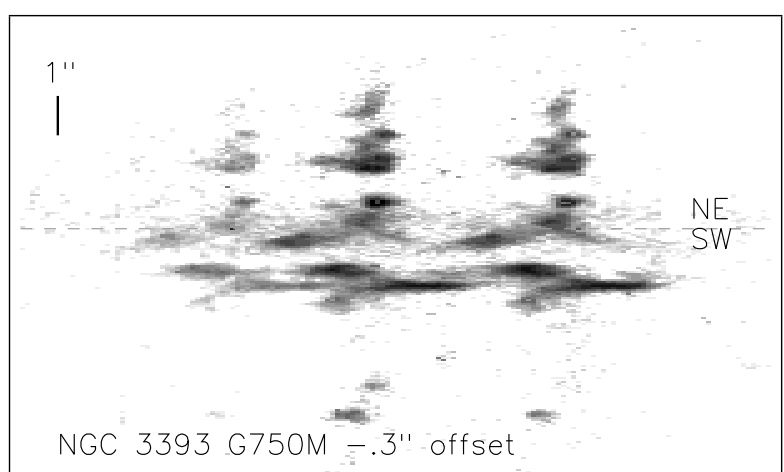

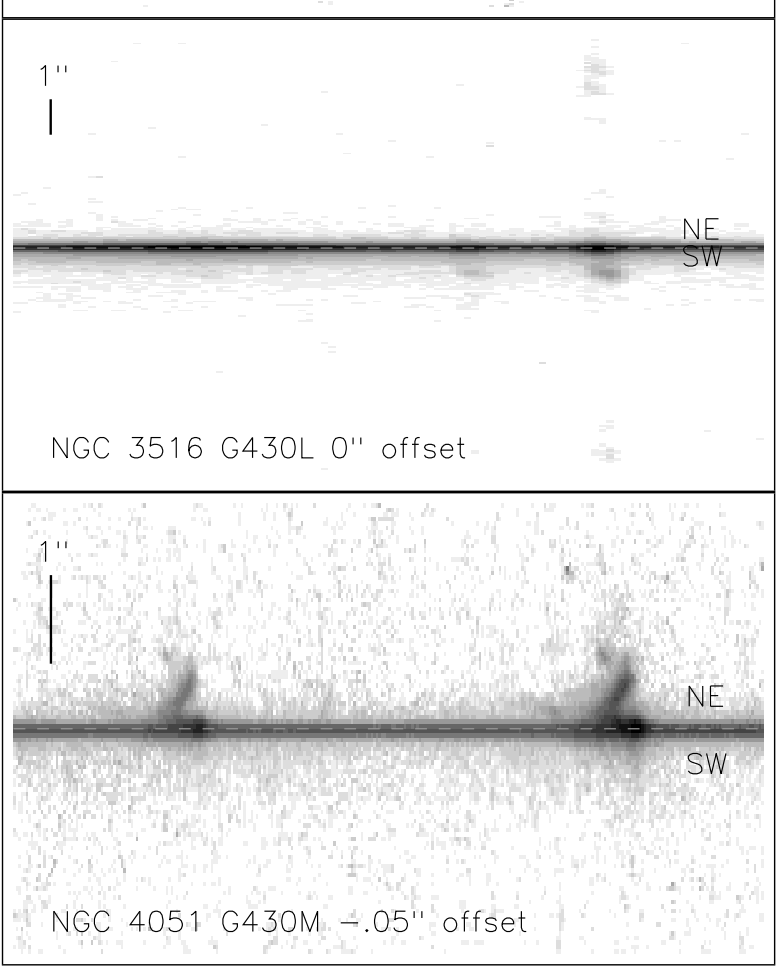

Figure B.10 

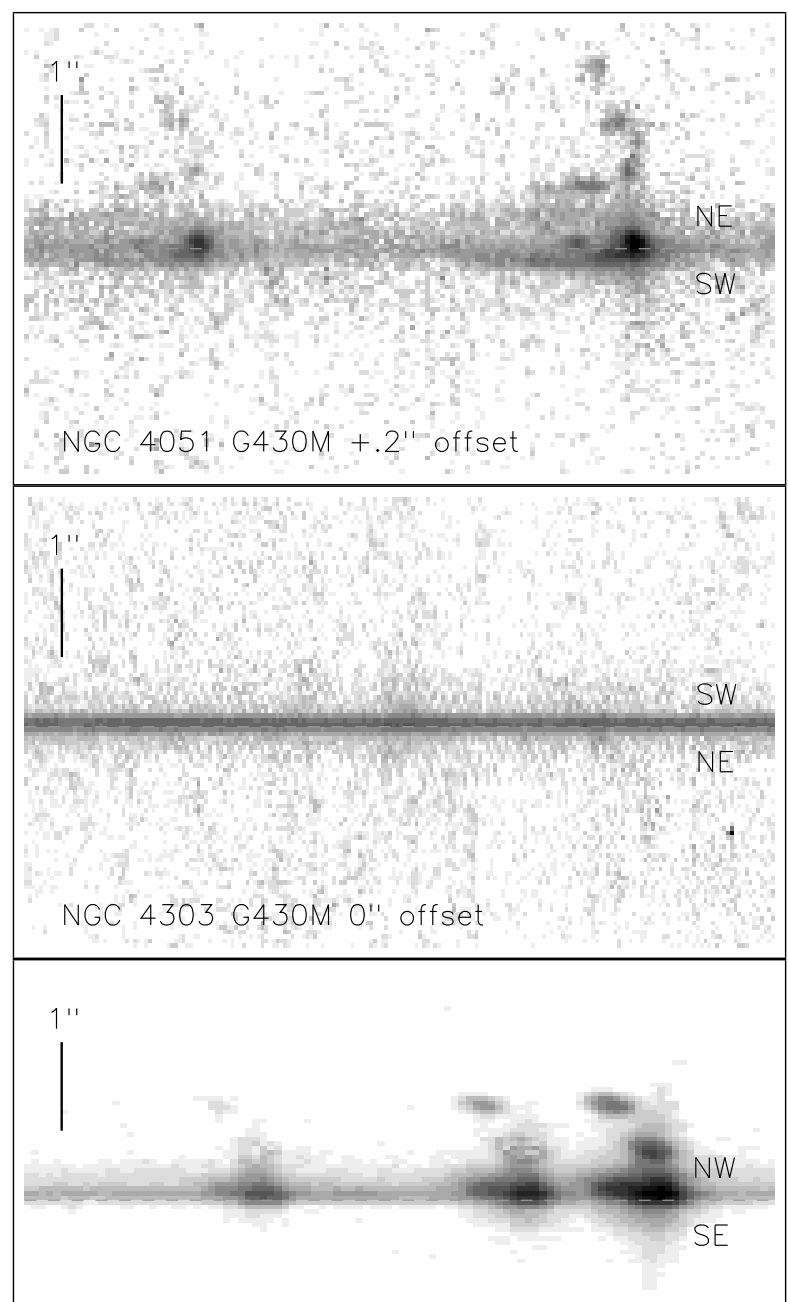

NGC 4507 G430L O" offset
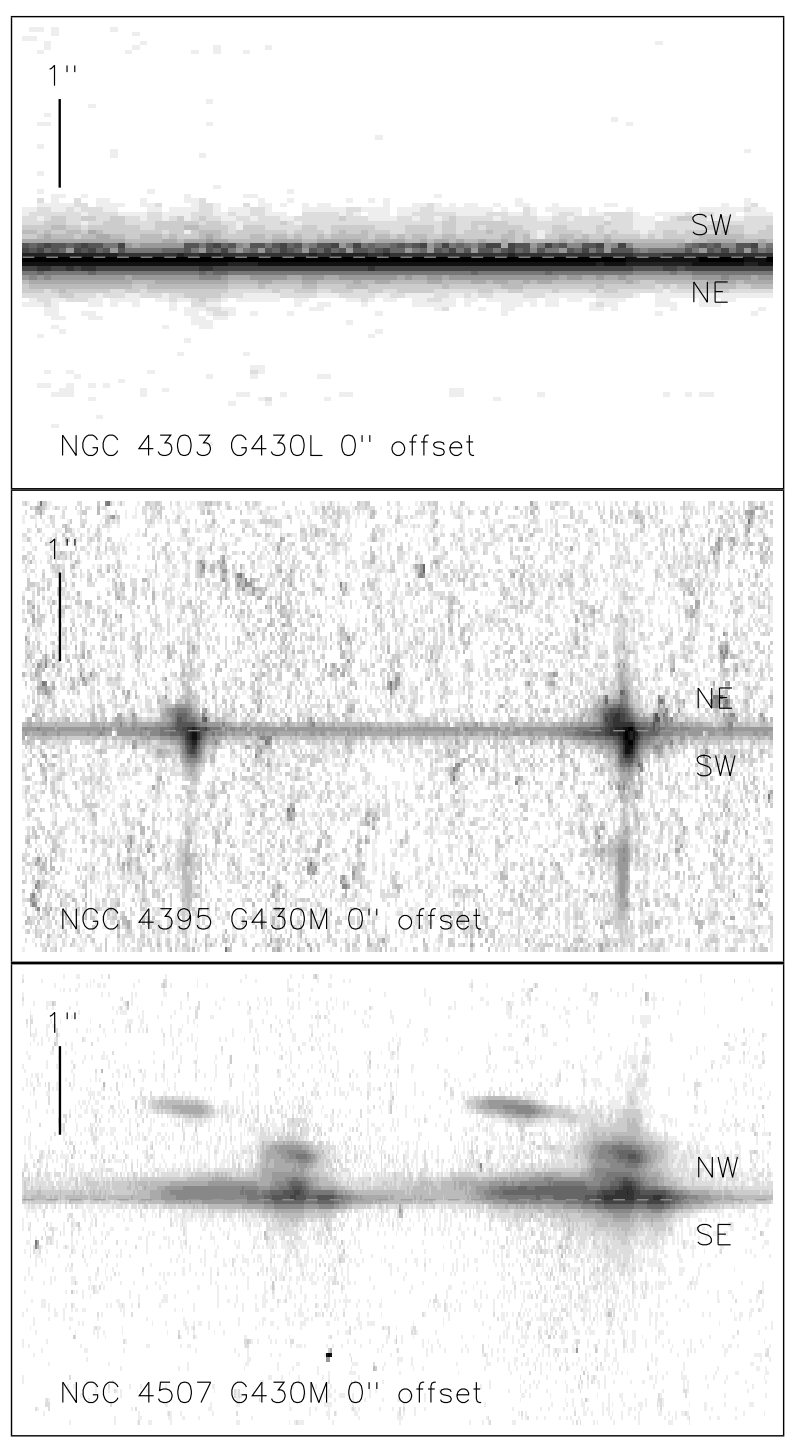

Figure B.11 


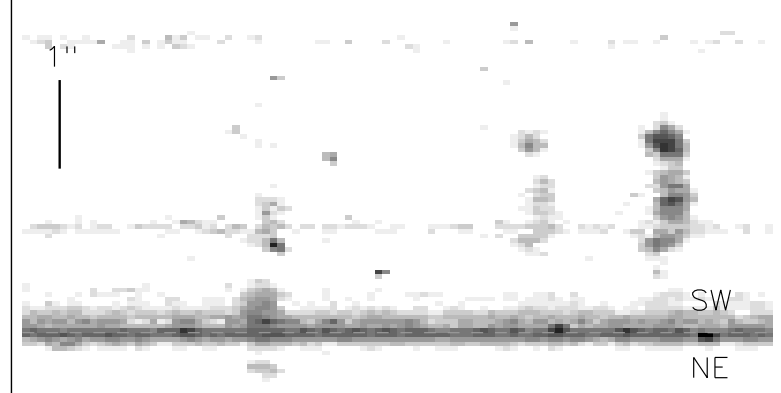

NGC 5135 G430L O" offset

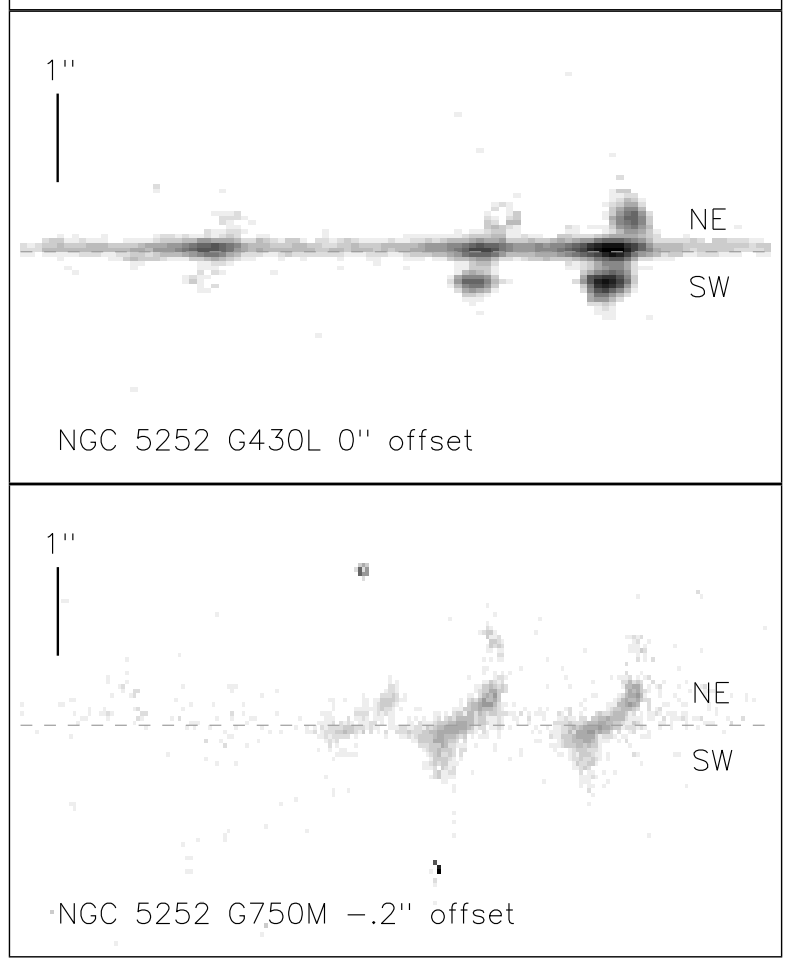

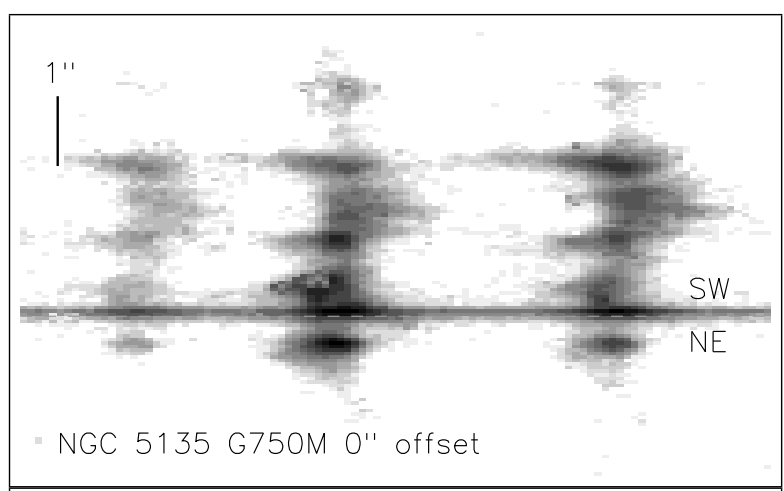

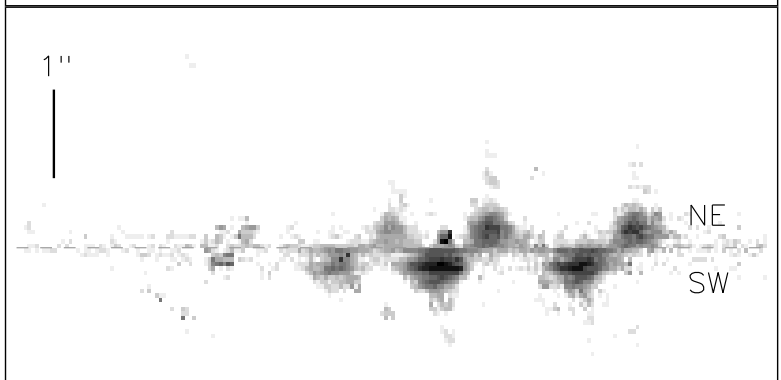

NGC 5252 G750M O" offset

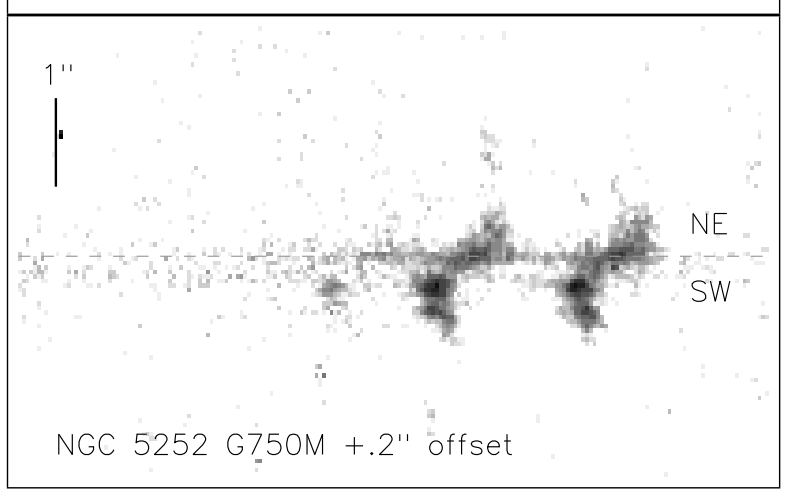

Figure B.12 

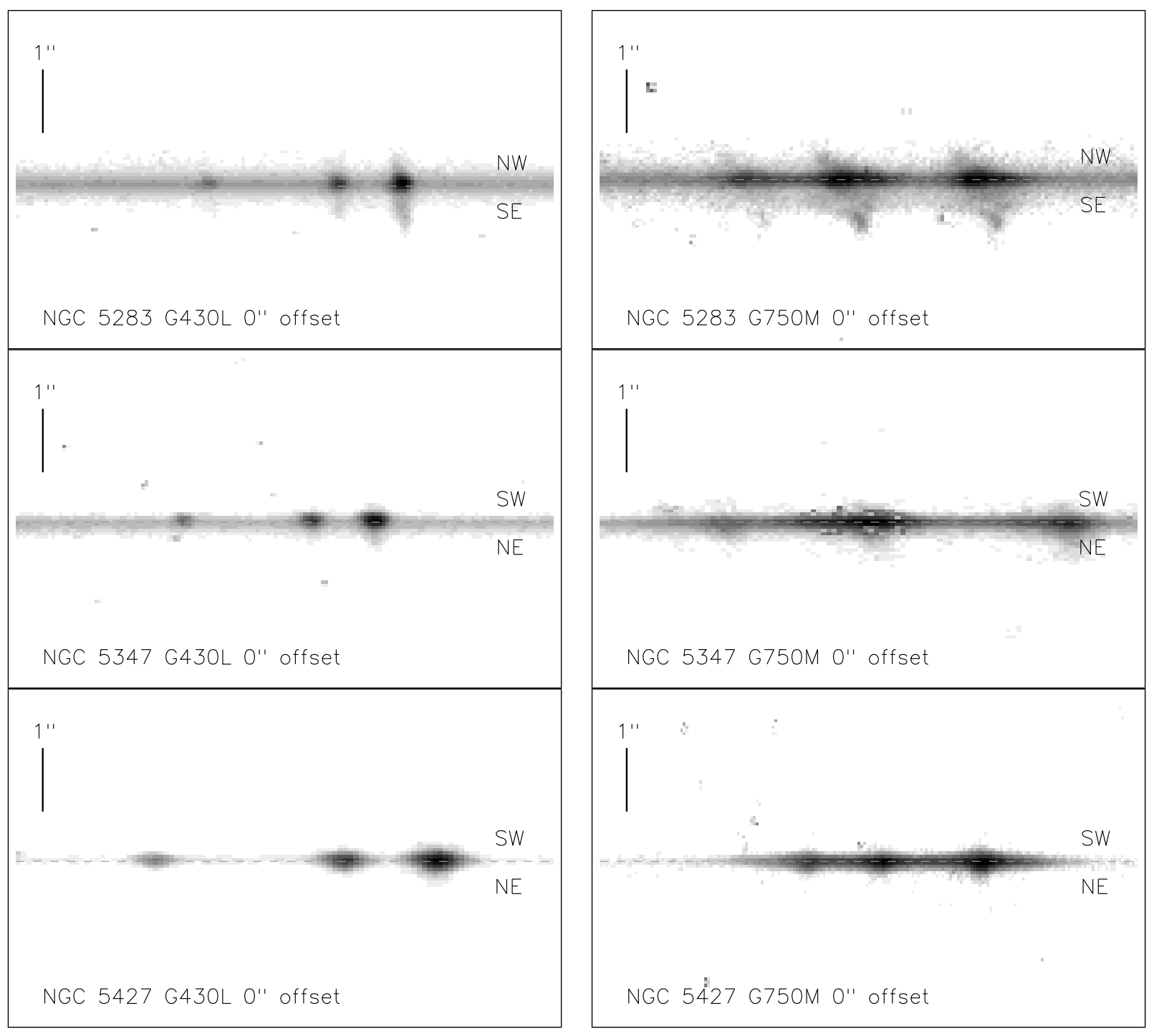

Figure B.13 

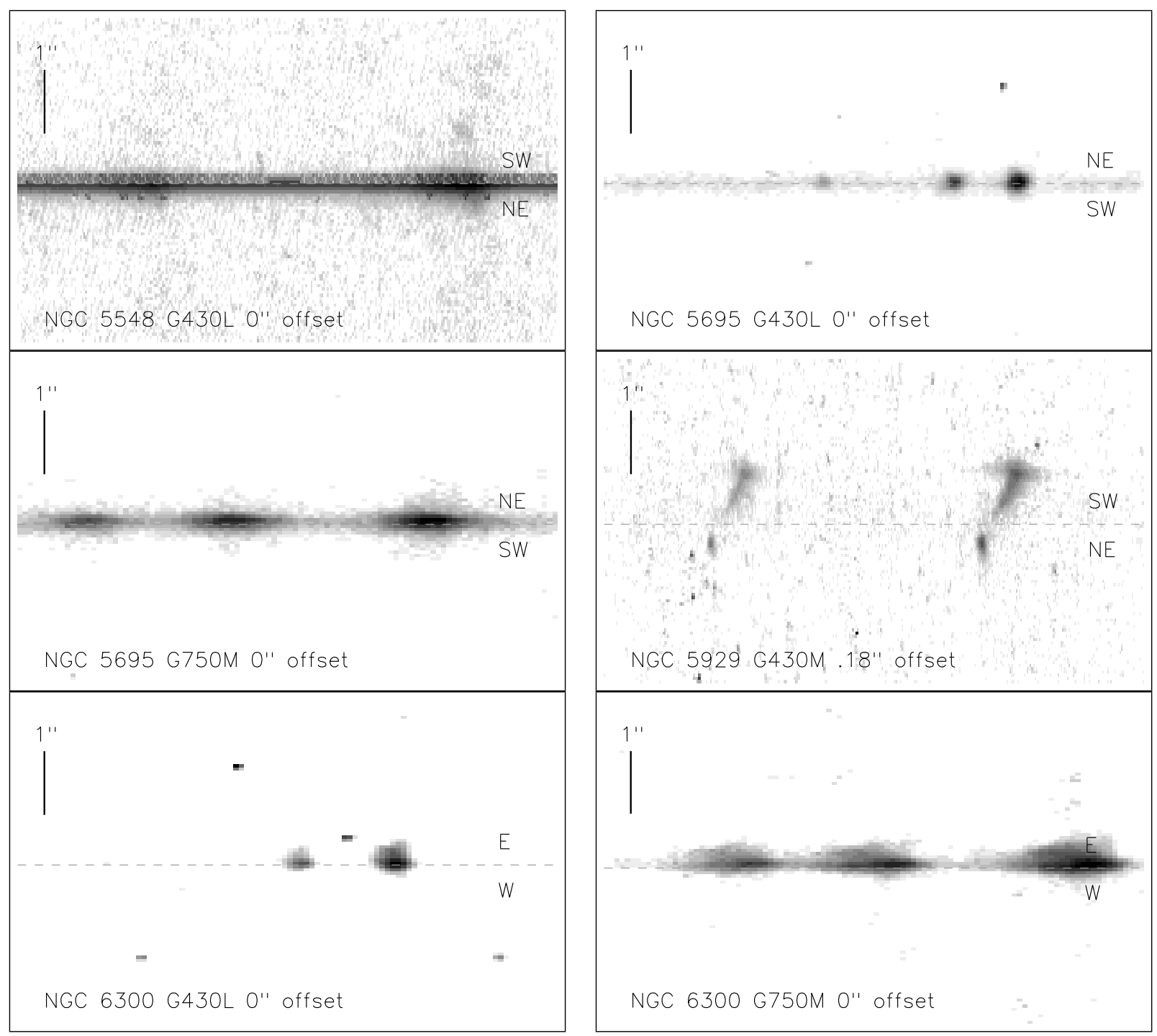

Figure B.14 

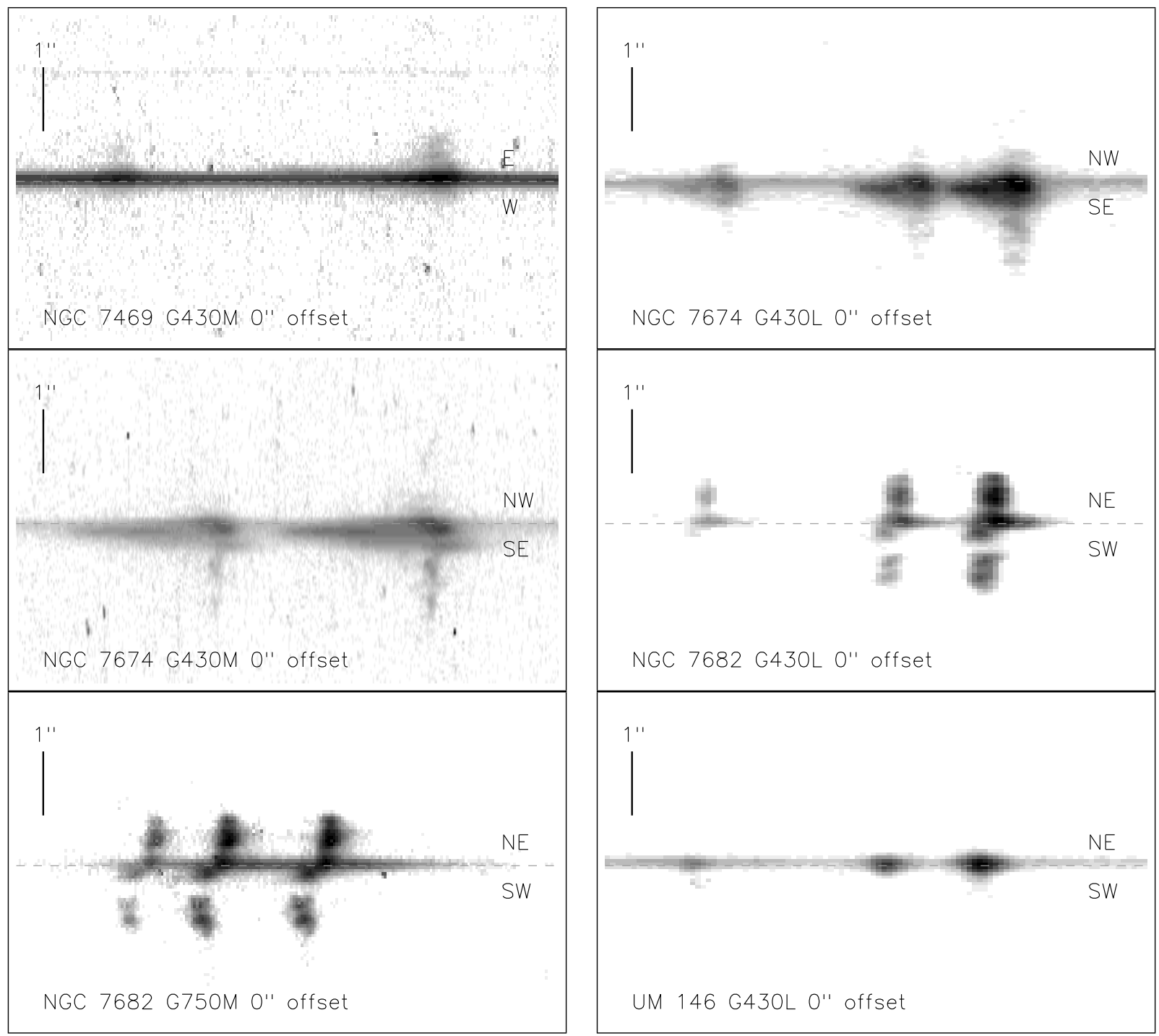

NGC 7682 G750M O" offset

NE

SW
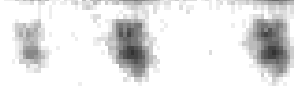

B.15 


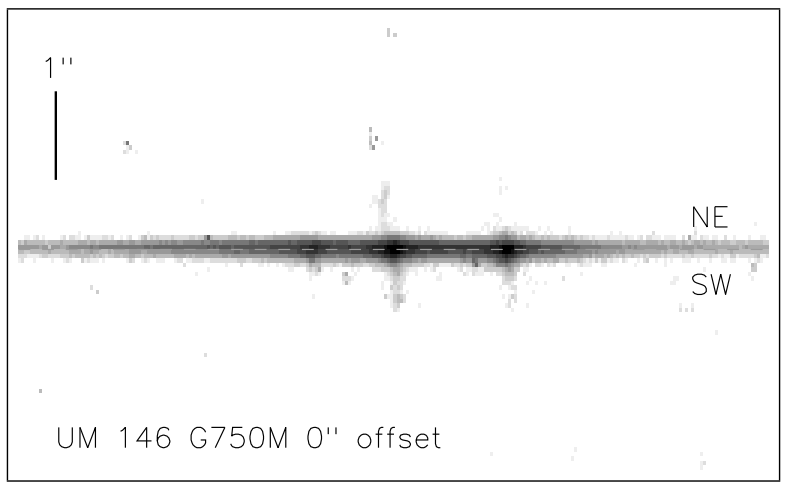

Figure B.16 


\section{APPENDIX C}

\section{Extended Sample: AGN Kinematics Data}

This appendix contains velocities, FWHMs, and normalized fluxes of each spectrum collected to created our expanded sample. Figures with multiple data sets signify observations using separate gratings at an identical position.

Kinematics data correspond to - HST gratings as follows:

Green Stars: [O III] $\lambda 5007$ emission line using G430L grating

Blue Triangles: [O III] $\lambda 5007$ emission line using G430M grating

Red Diamonds: H $\alpha$ 入6564 emission line using G750M grating 


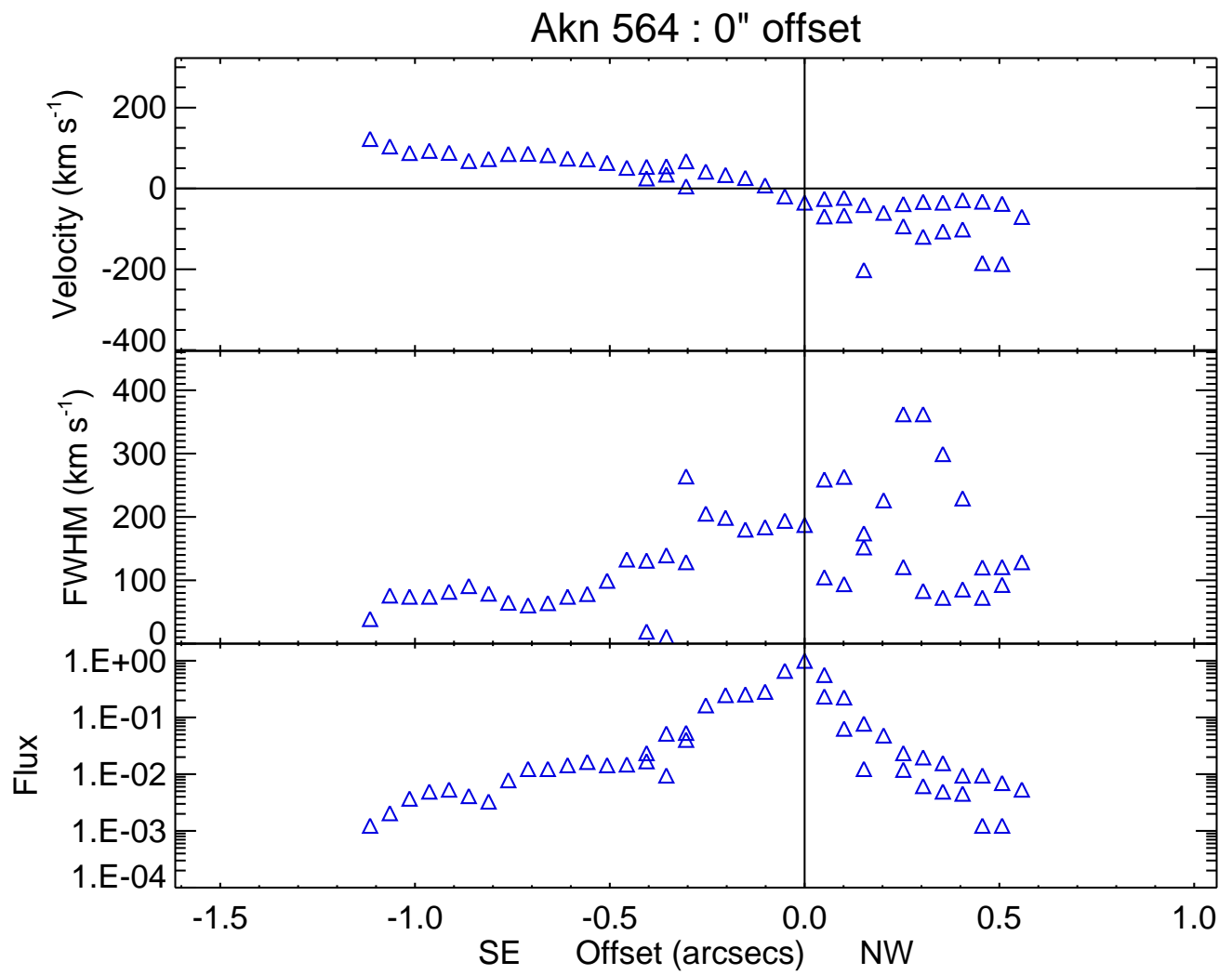

Circinus : - .6 " offset

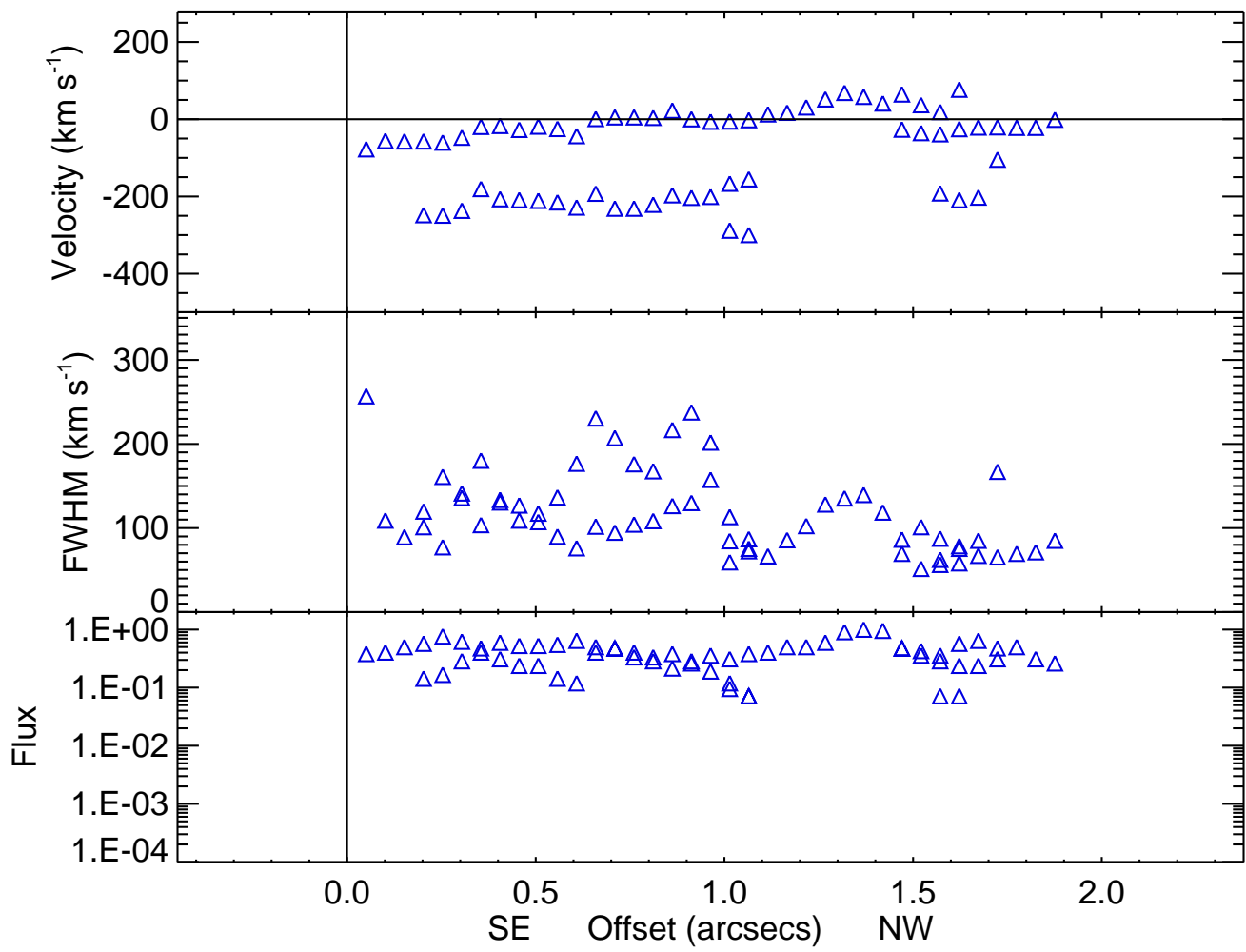

Figure C.1 
Circinus : -.4 " offset

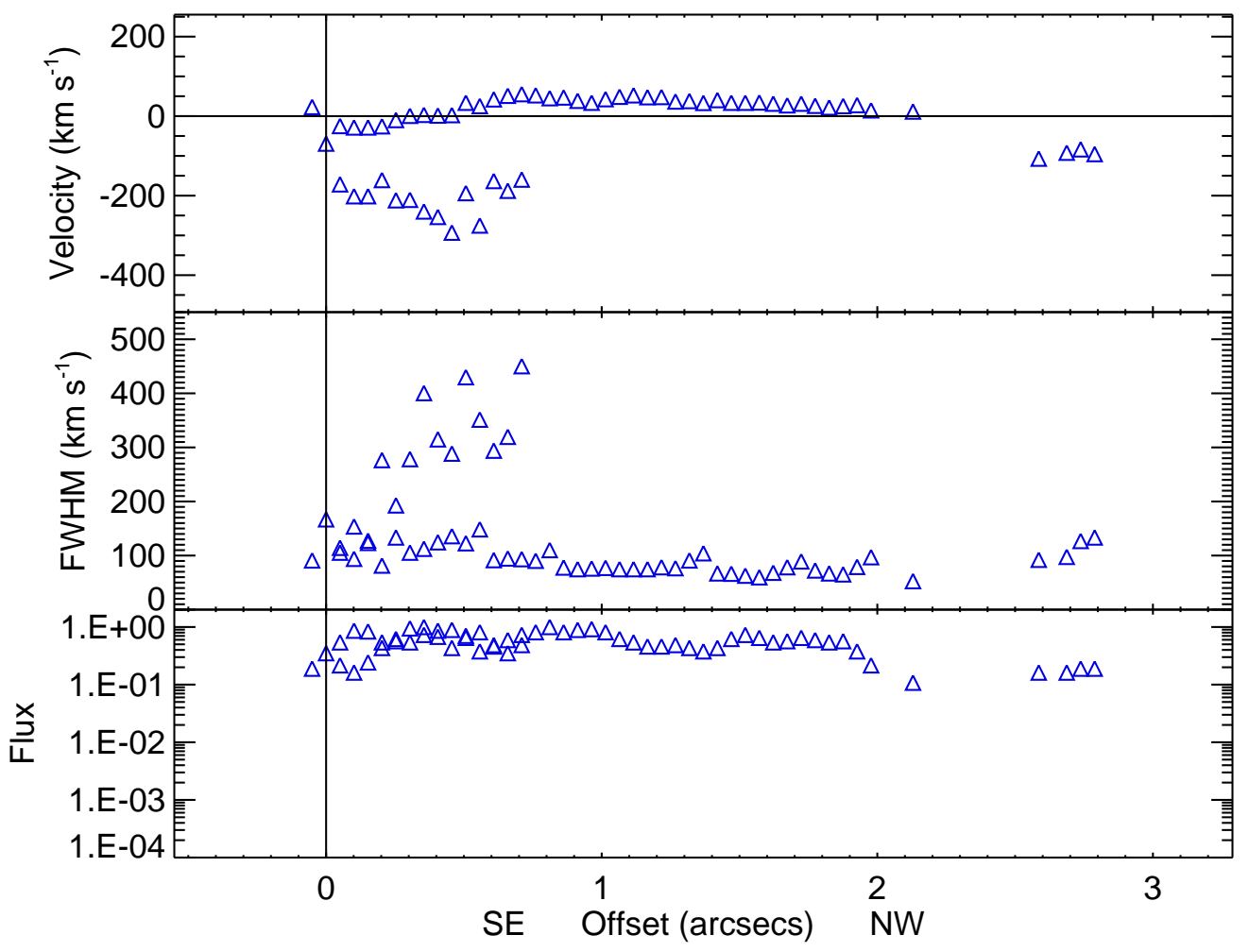

Circinus : -.2" offset

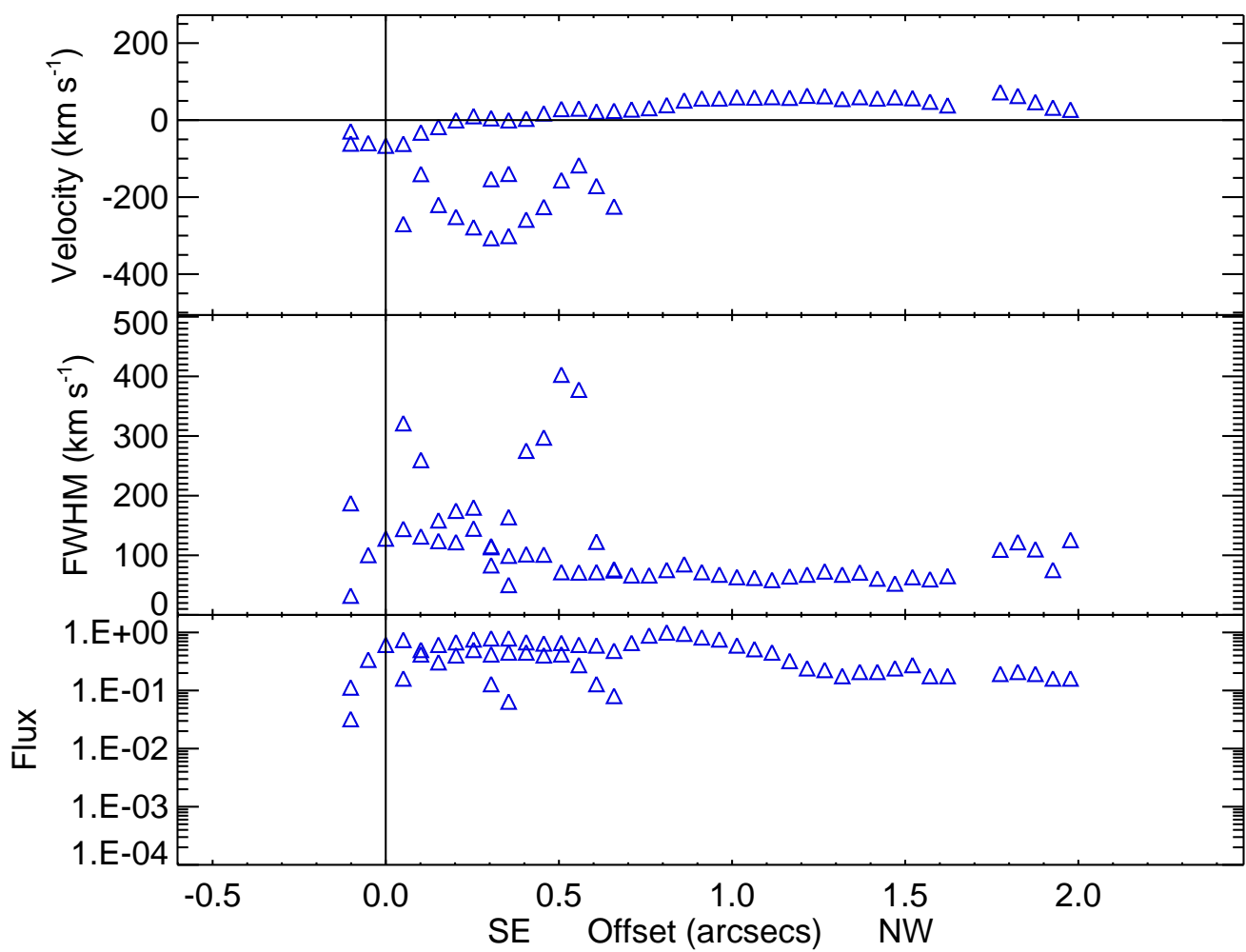

Figure C.2 
Circinus : 0" offset

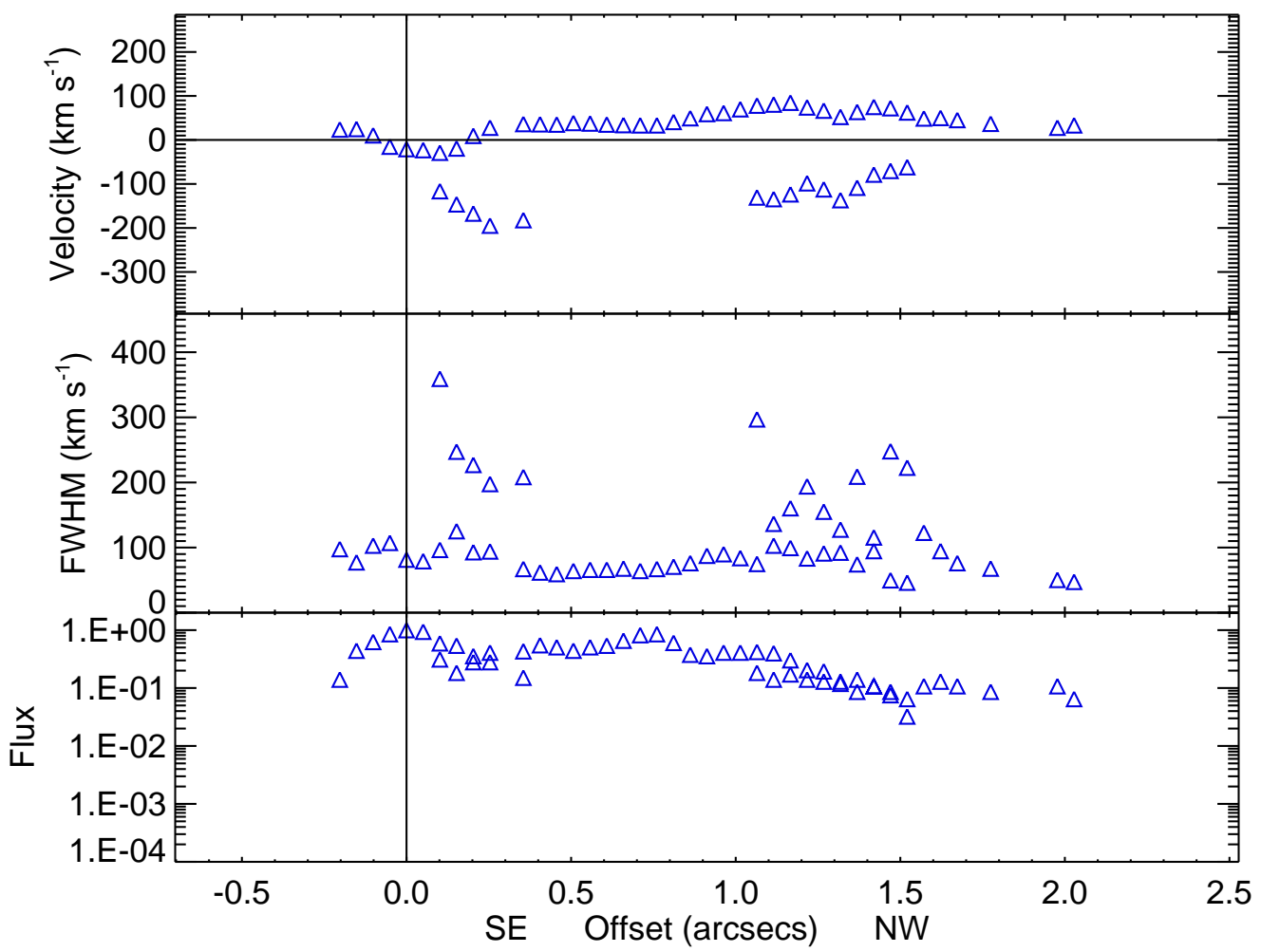

Circinus : +.2 " offset

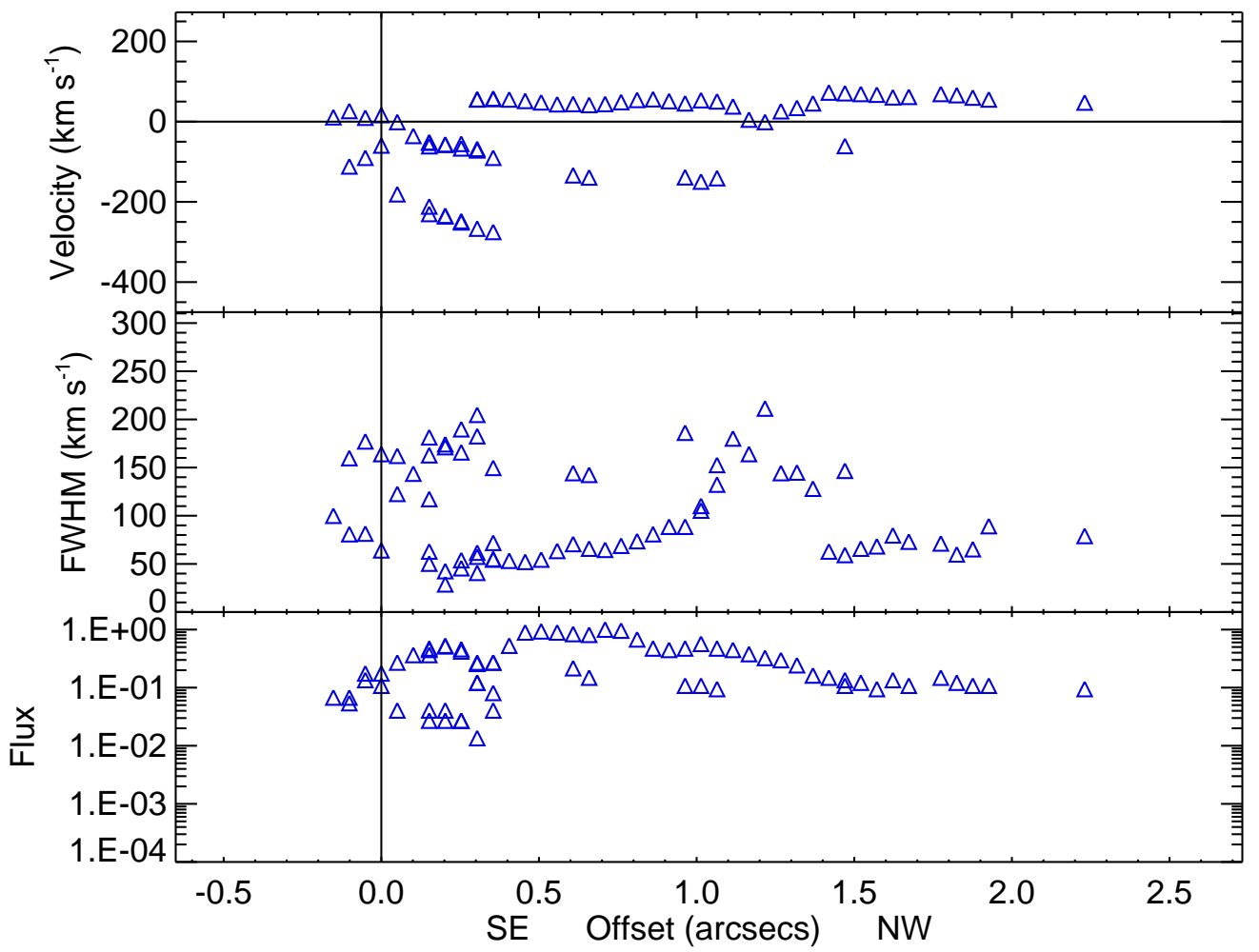

Figure C.3 

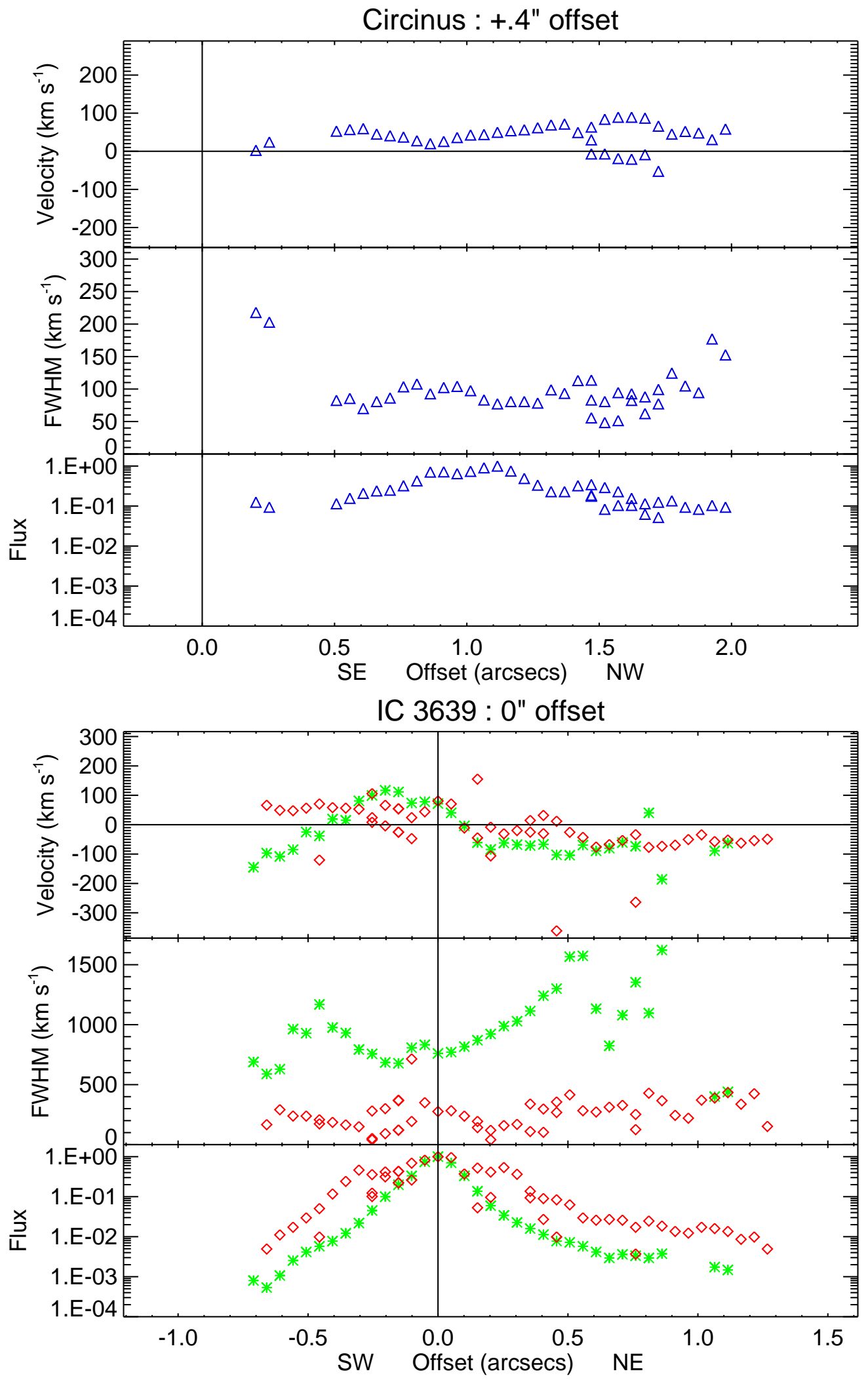

Figure C.4 
IRAS 11058-1131:0" offset
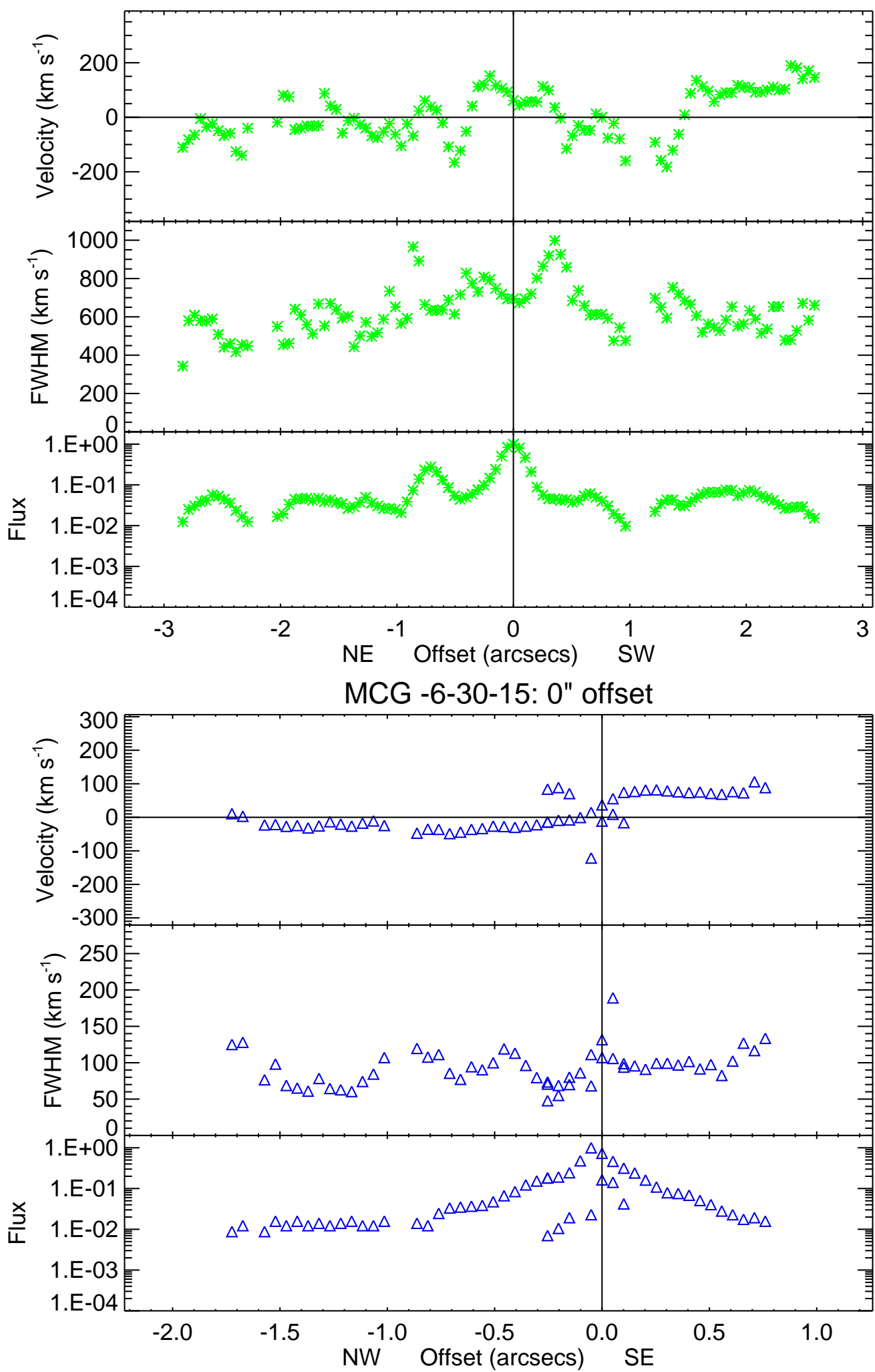

Figure C.5 

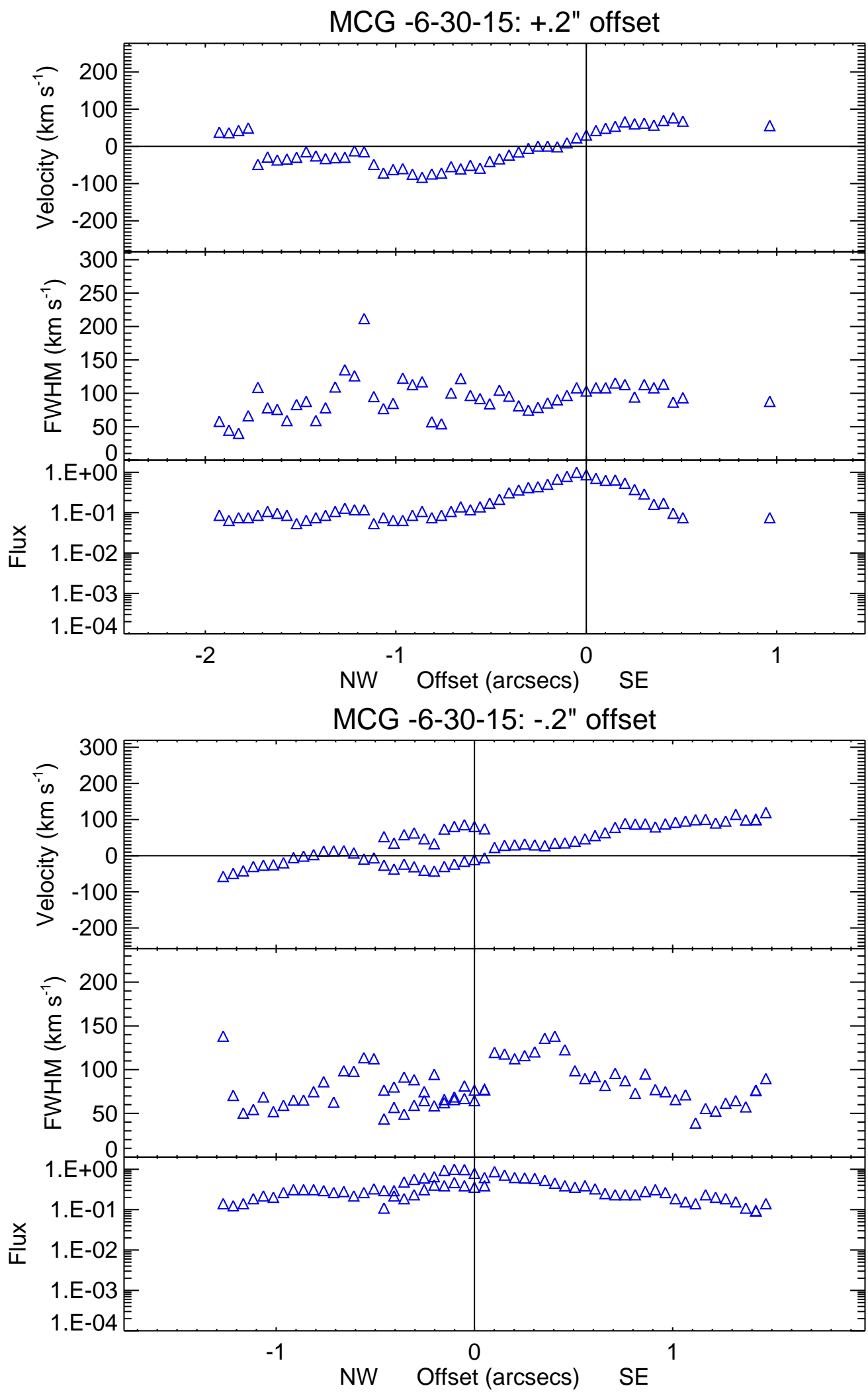

Figure C.6 
Mrk 34 : 0" offset

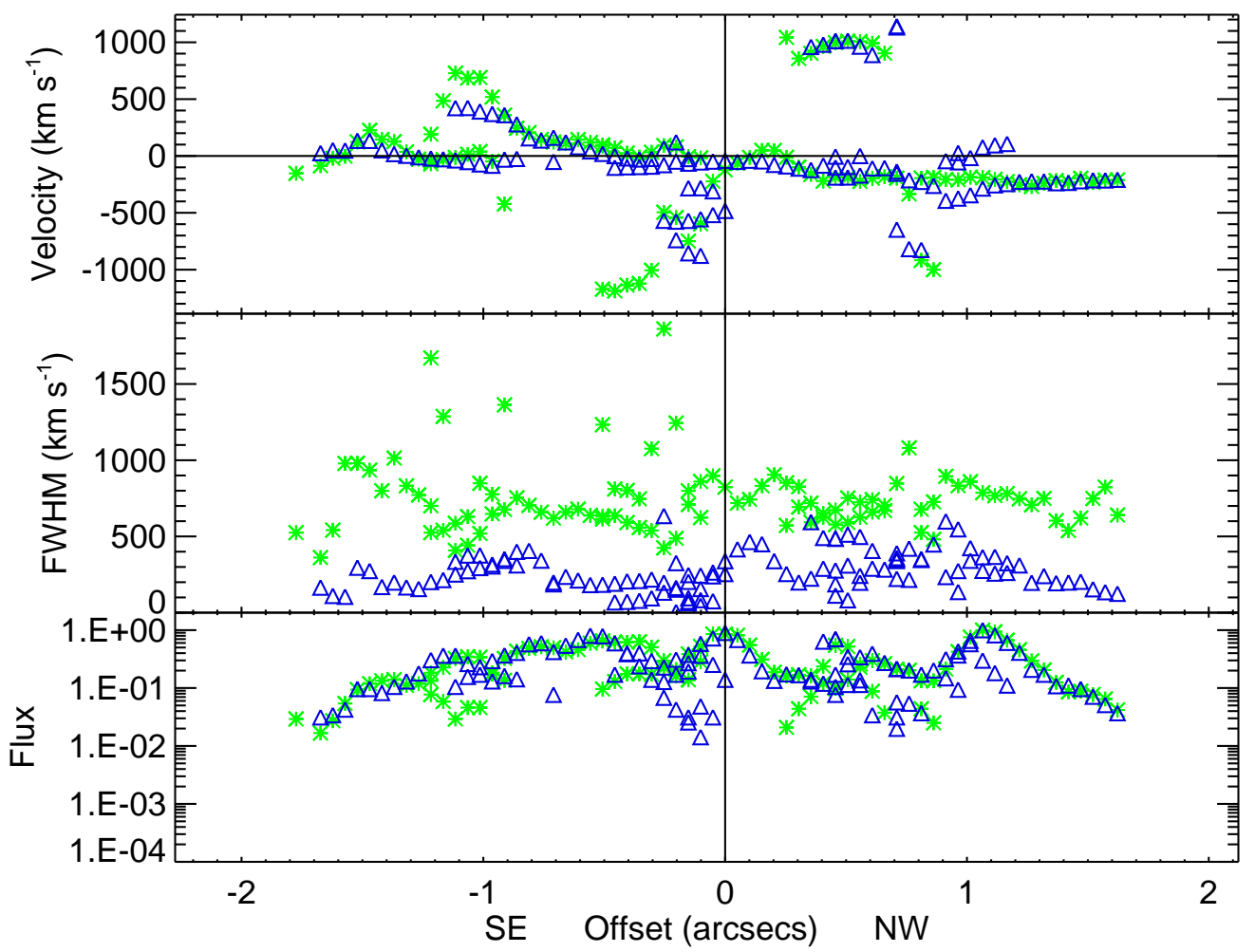

Mrk $34:+.28$ " offset

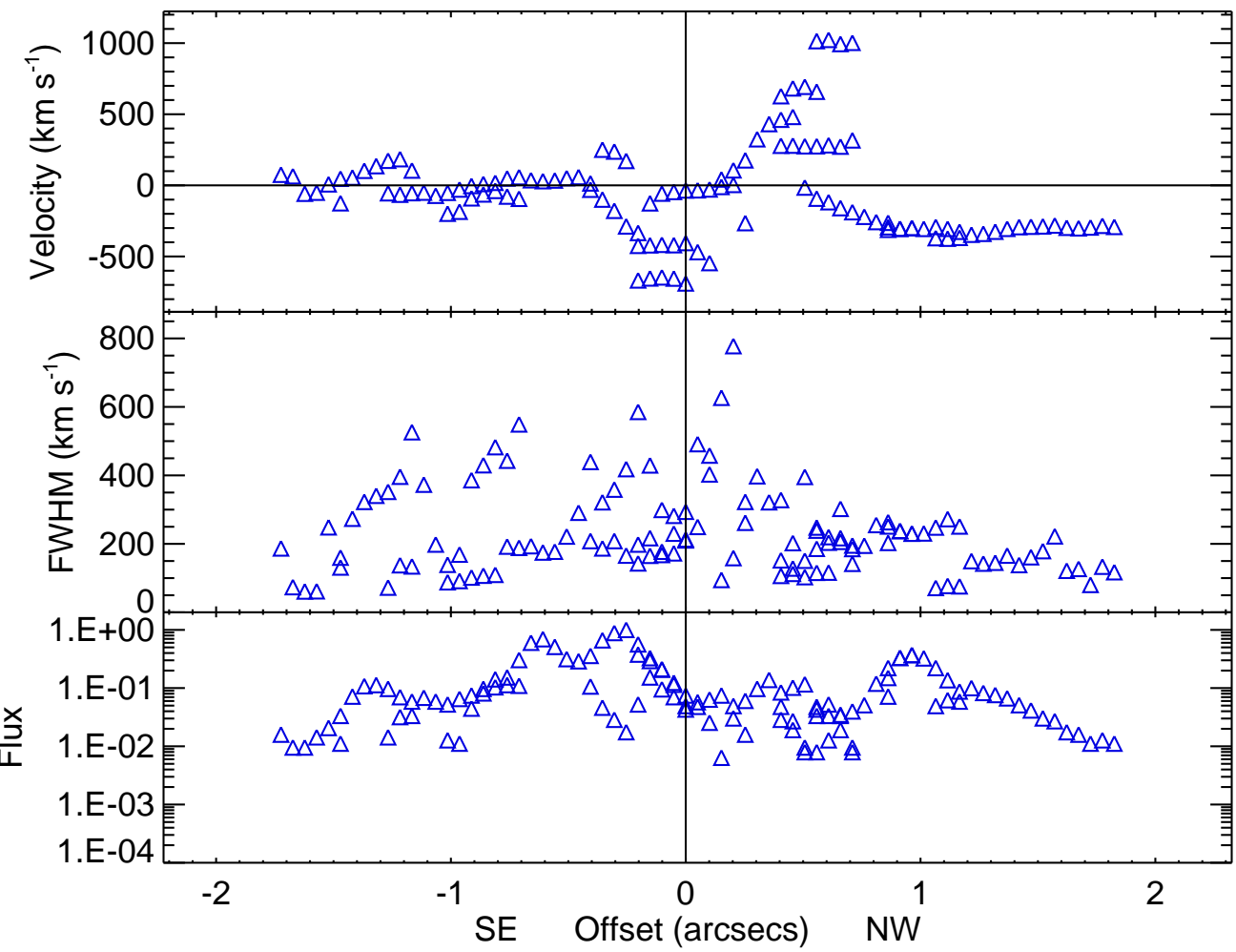

Figure C.7 
Mrk 34 : -.28" offset

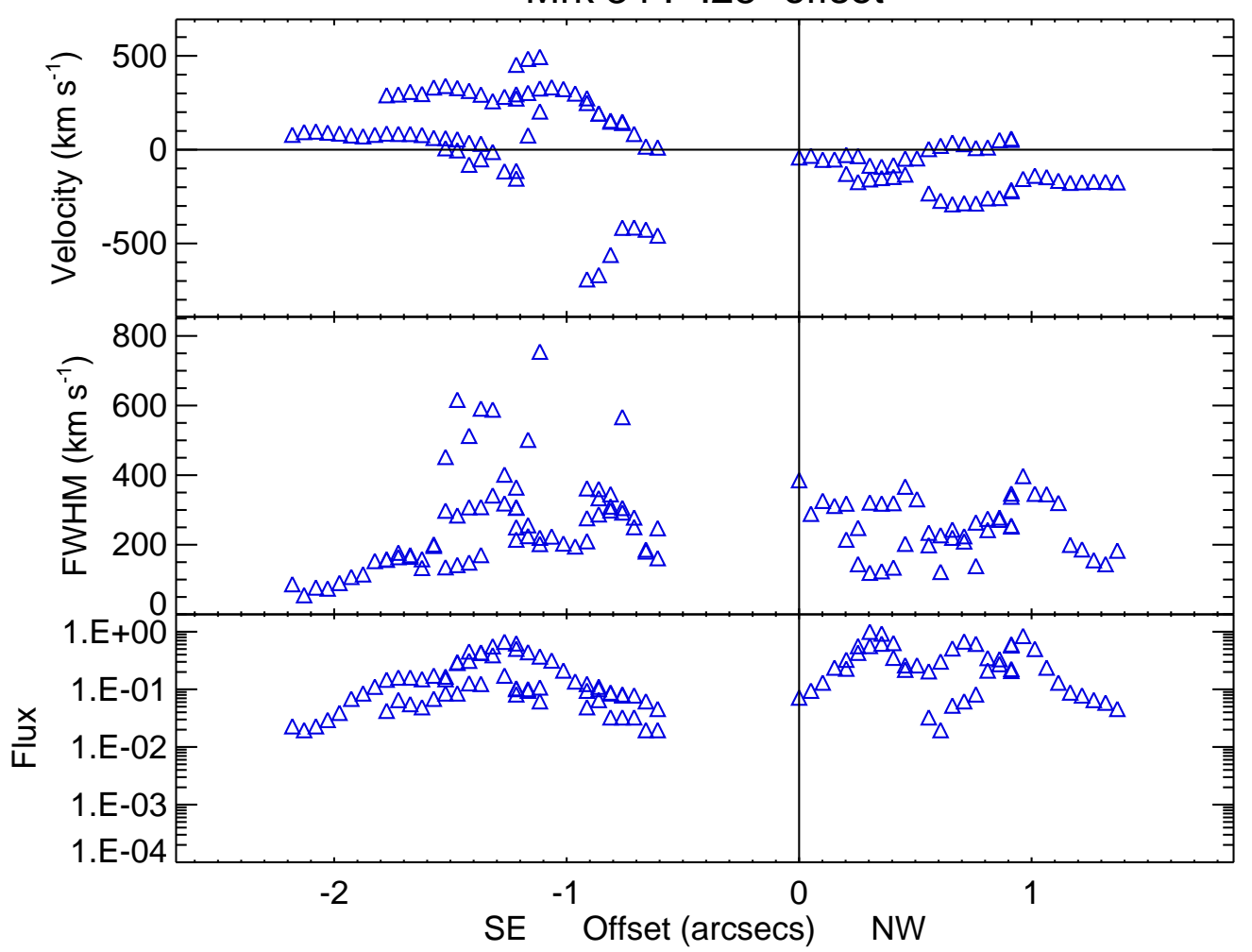

Mrk 279 : 0" offset

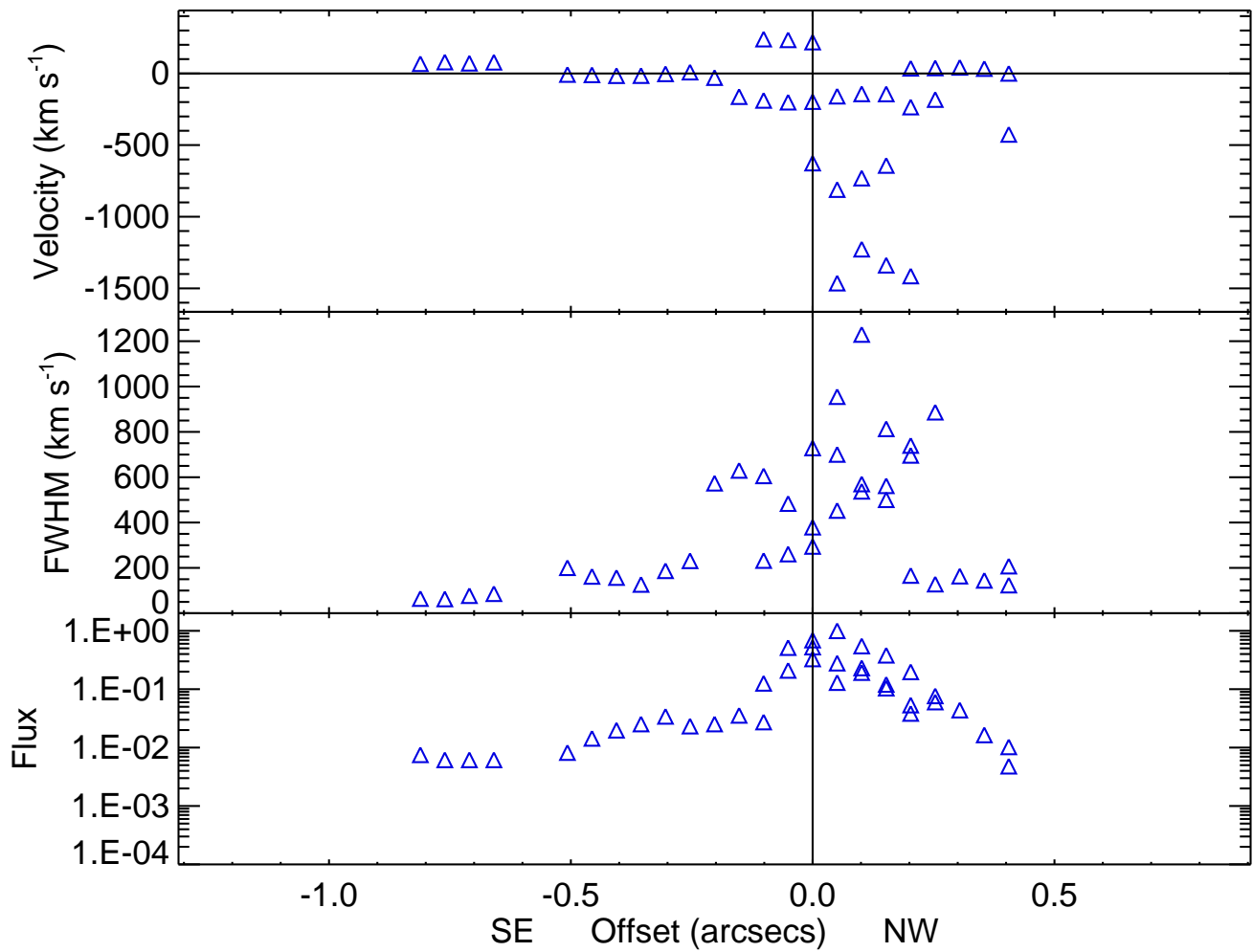

Figure C.8 
Mrk 348:0" offset

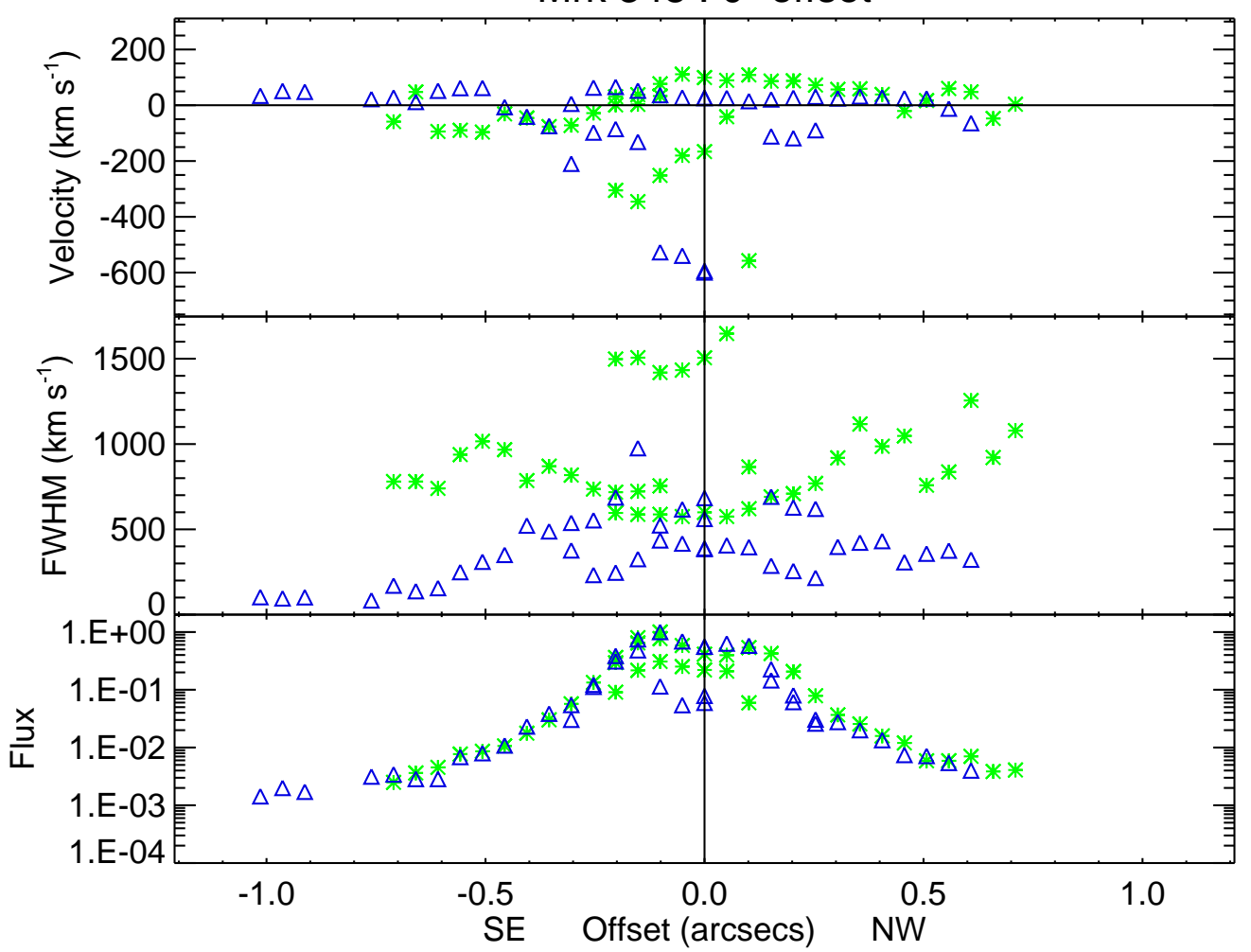

Mrk 463e : 0" offset

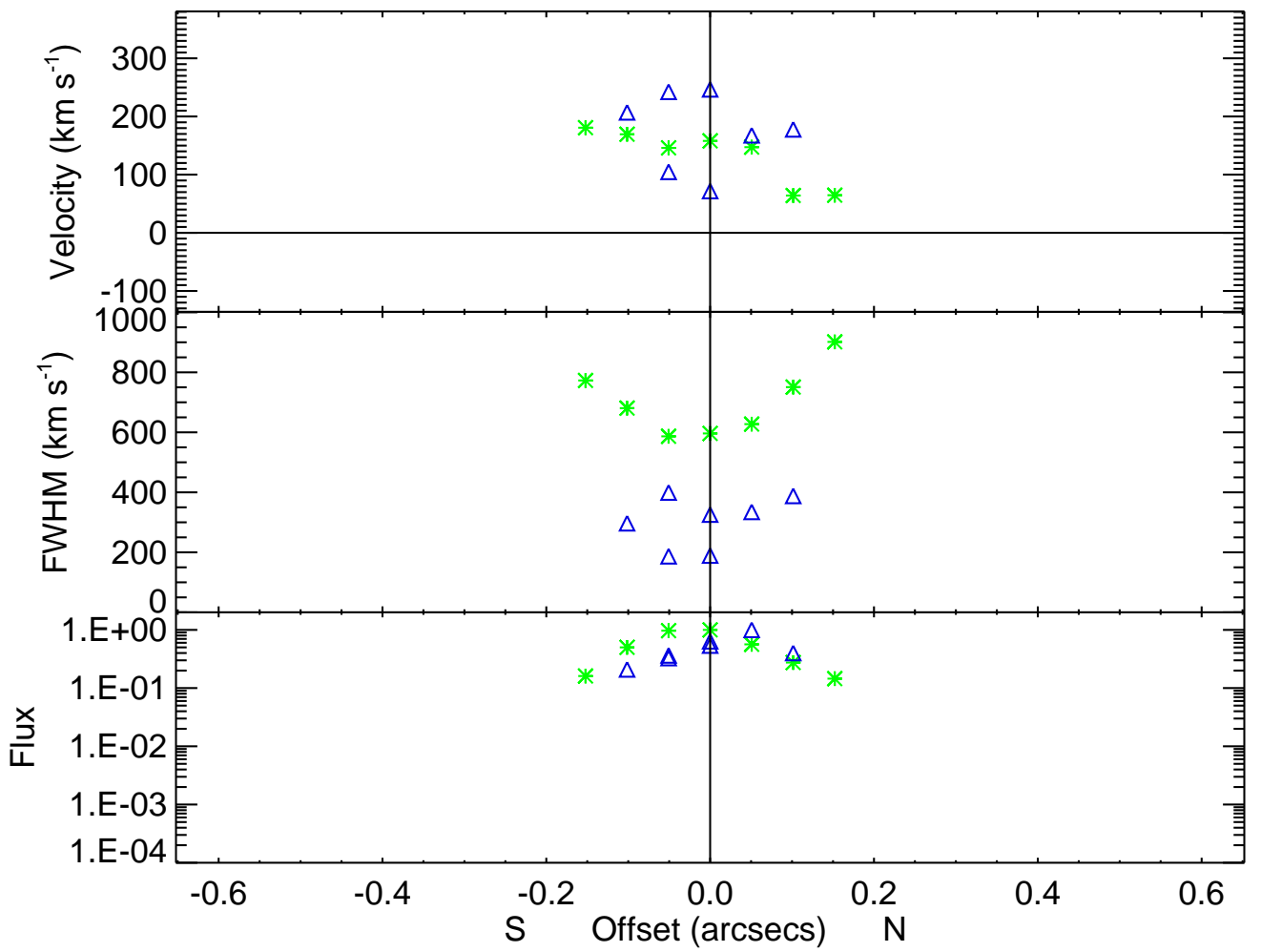

Figure C.9 
Mrk 493 : 0" offset

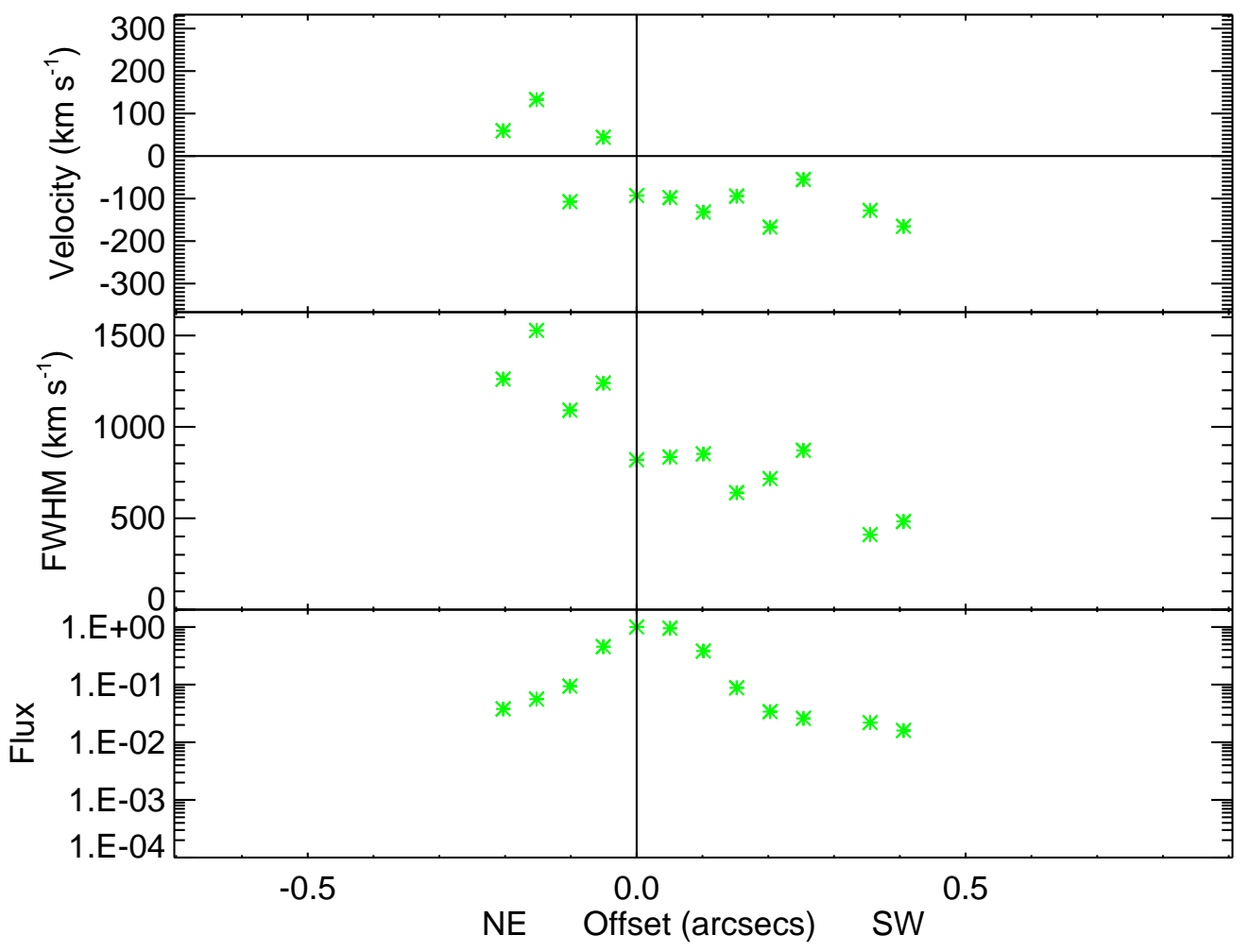

Mrk 509 : 0" offset

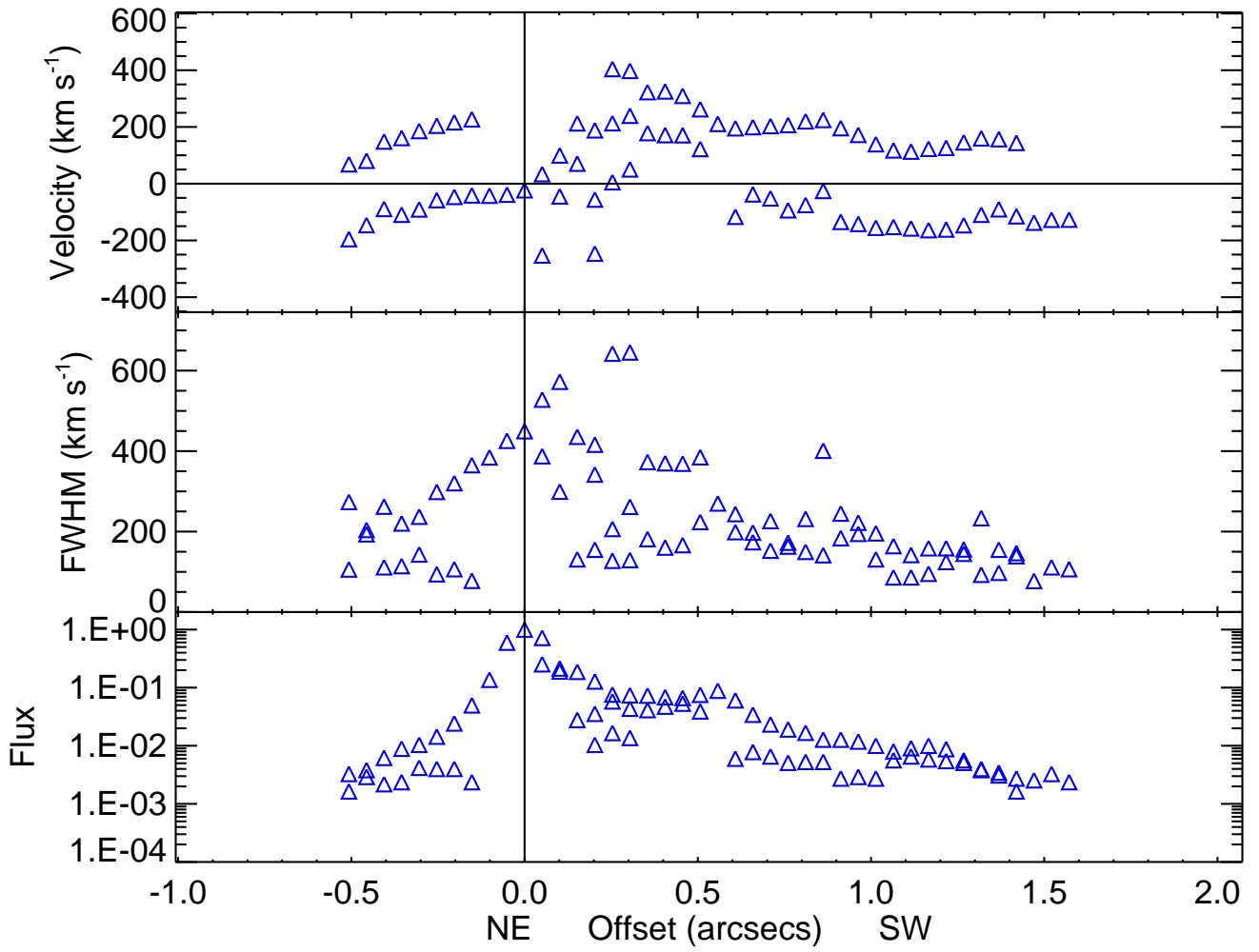

Figure C.10 
Mrk $705: 0 "$ offset

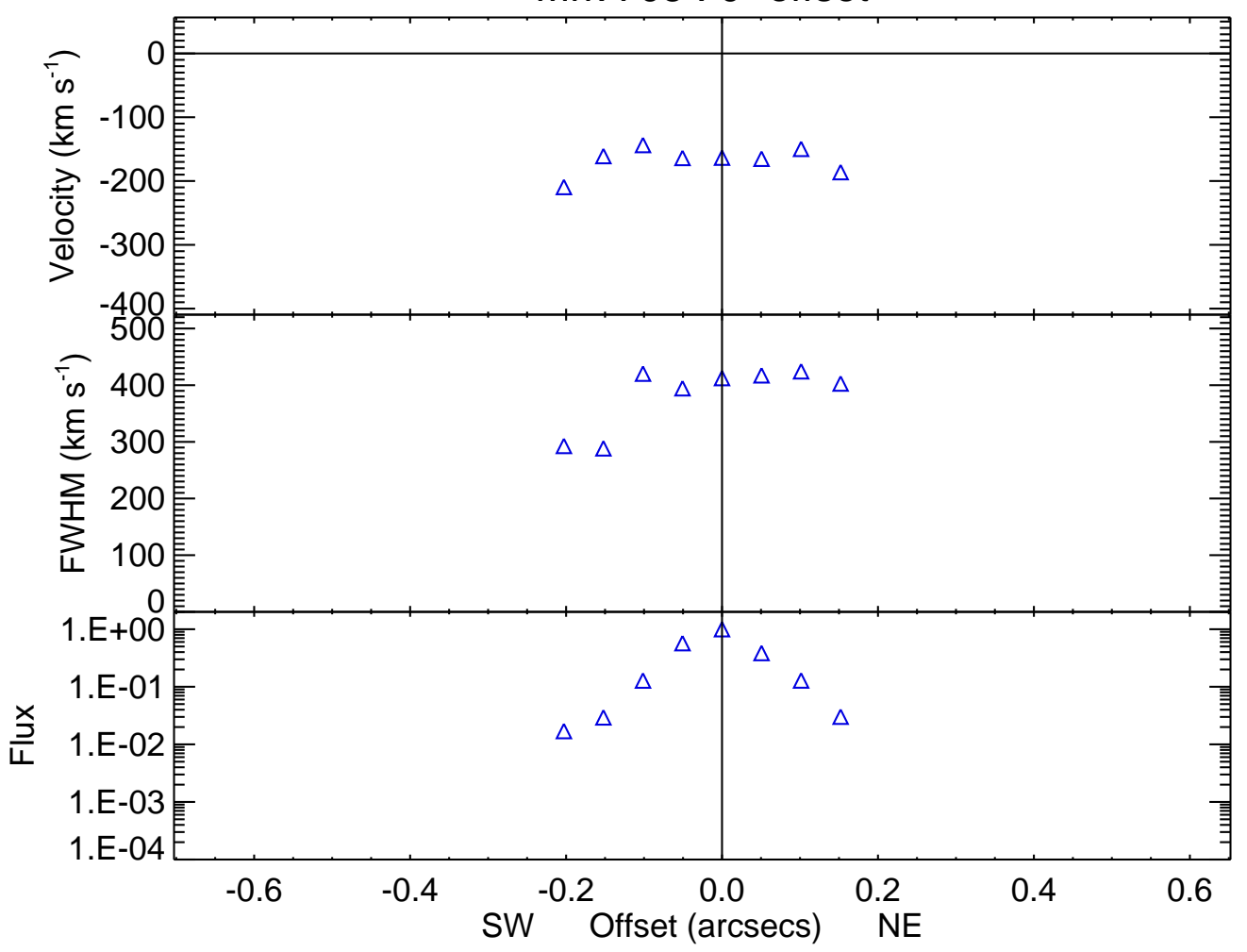

Mrk $705: 0$ " offset

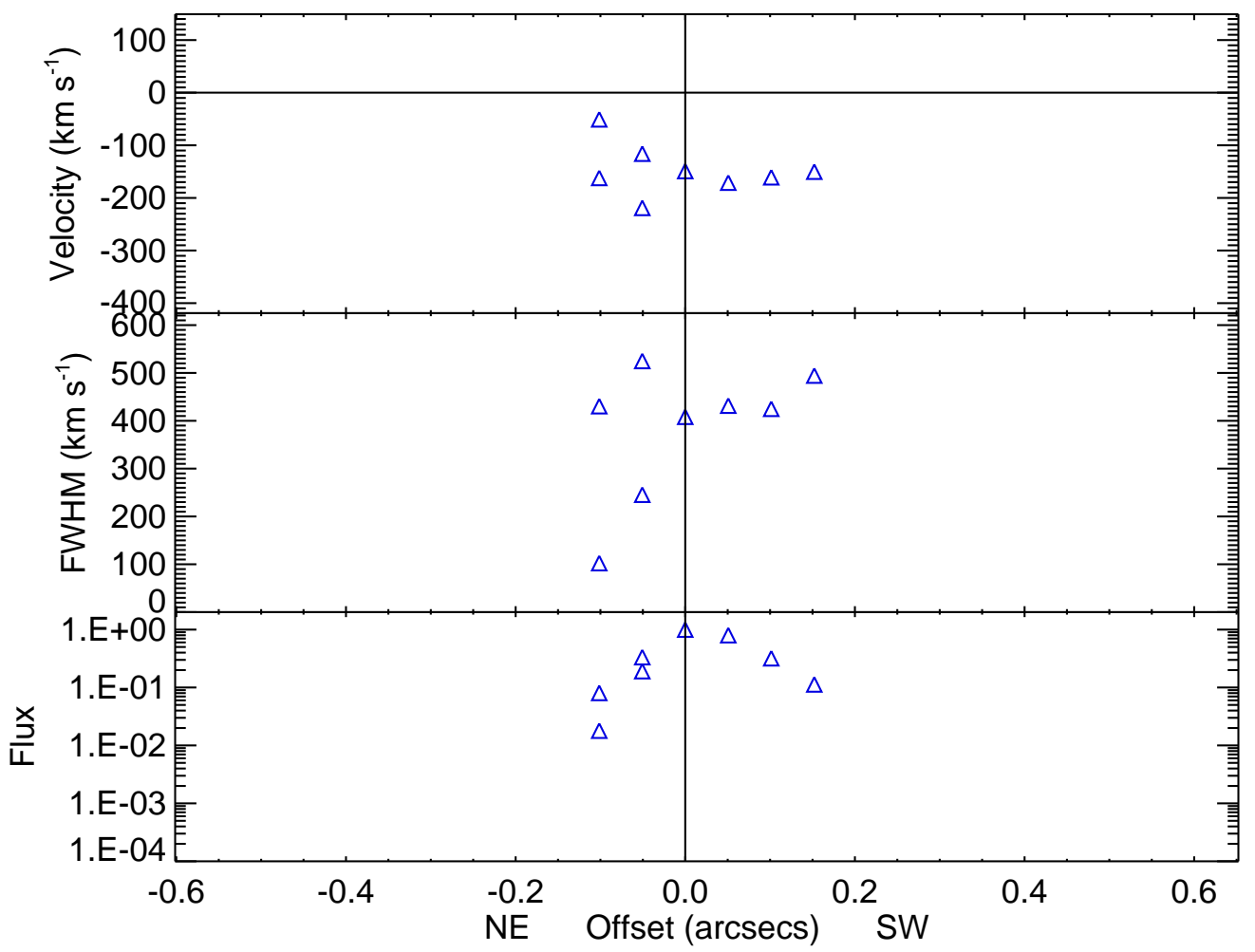

Figure C.11 Top: STIS slit P.A. $=-100.35$, Bottom: STIS slit P.A. $=7.92$ 
Mrk $766: 0 "$ offset

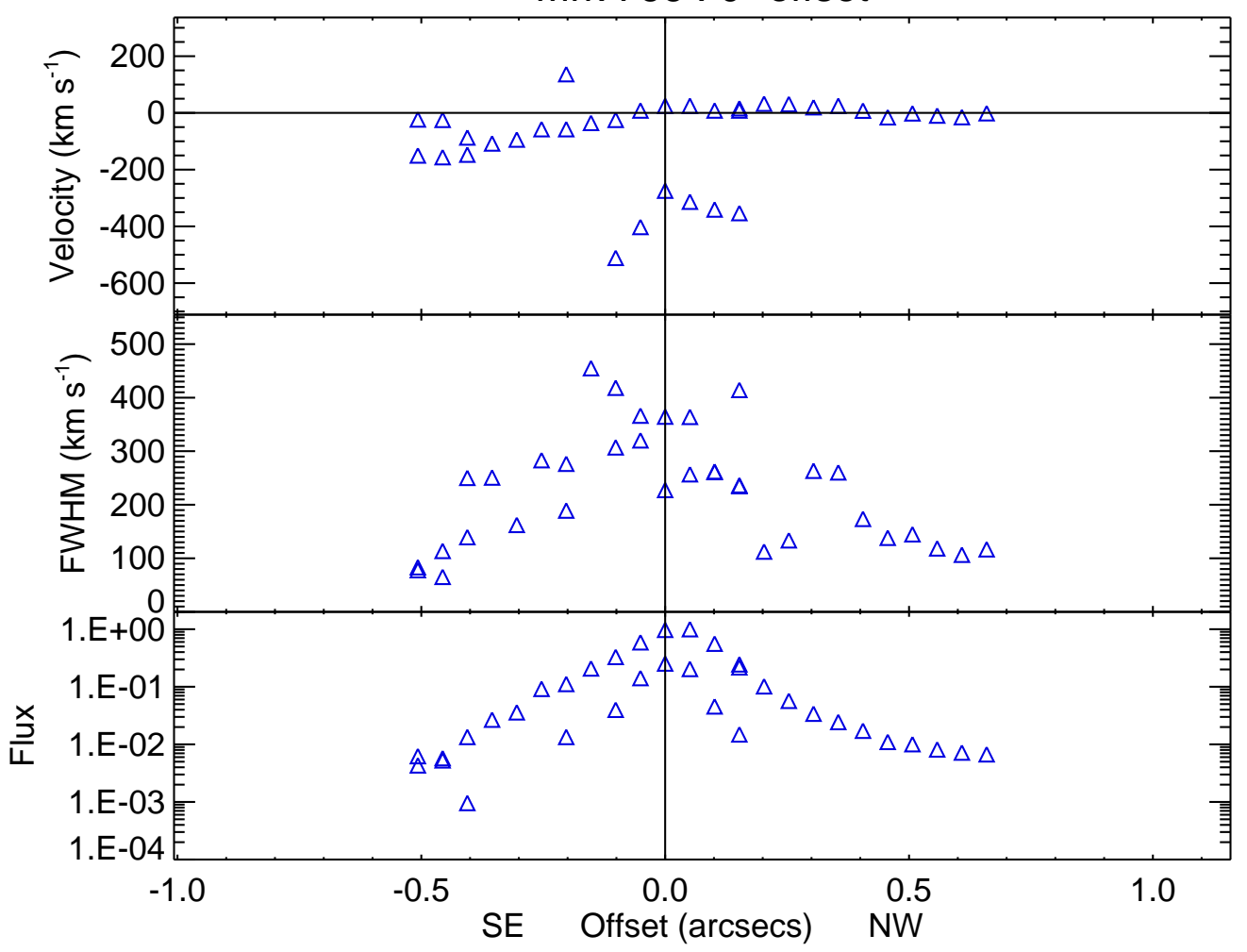

Mrk $766: 0$ " offset

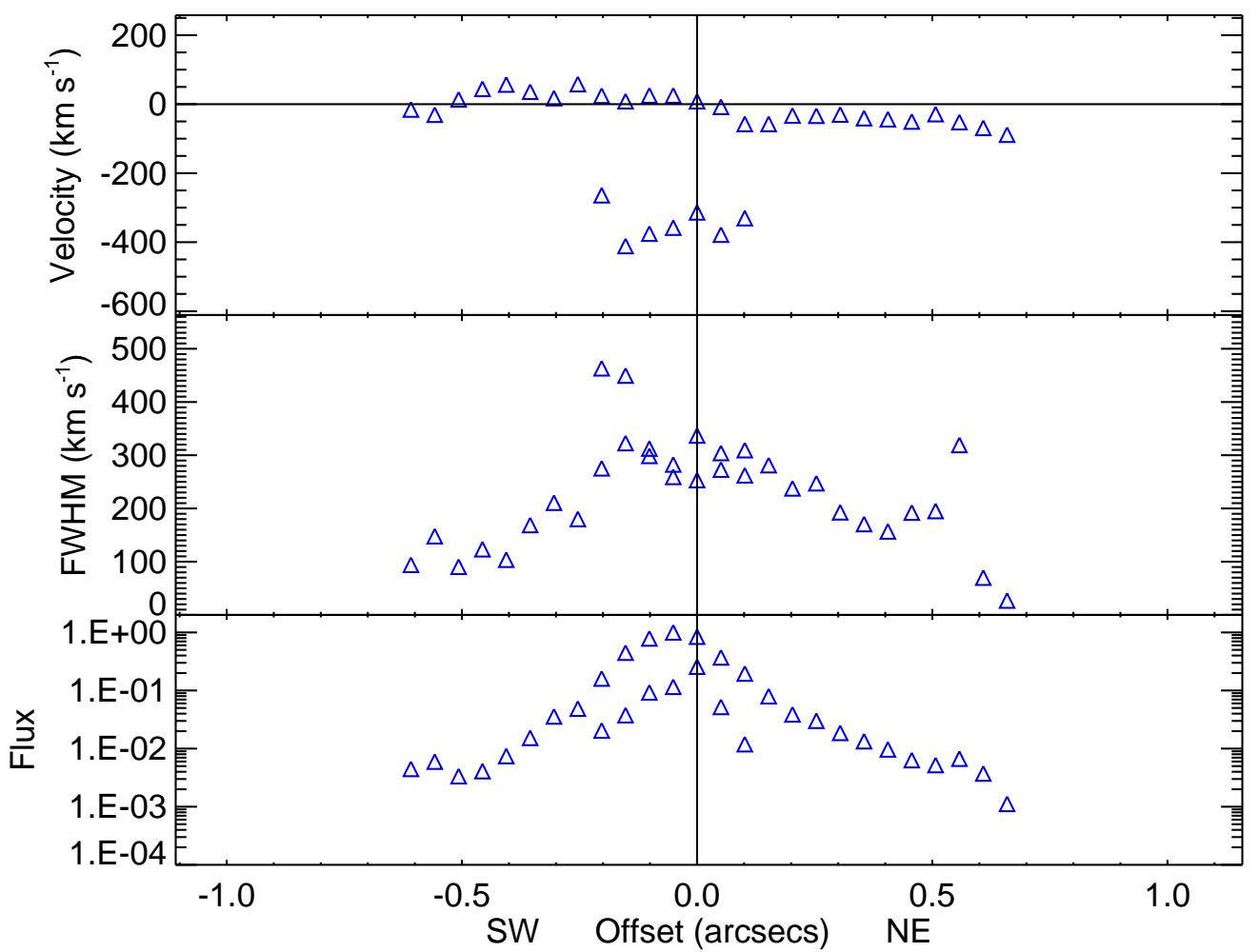

Figure C.12 Top: STIS slit P.A. $=129.65$, Bottom: STIS slit P.A. $=-120.35$ 

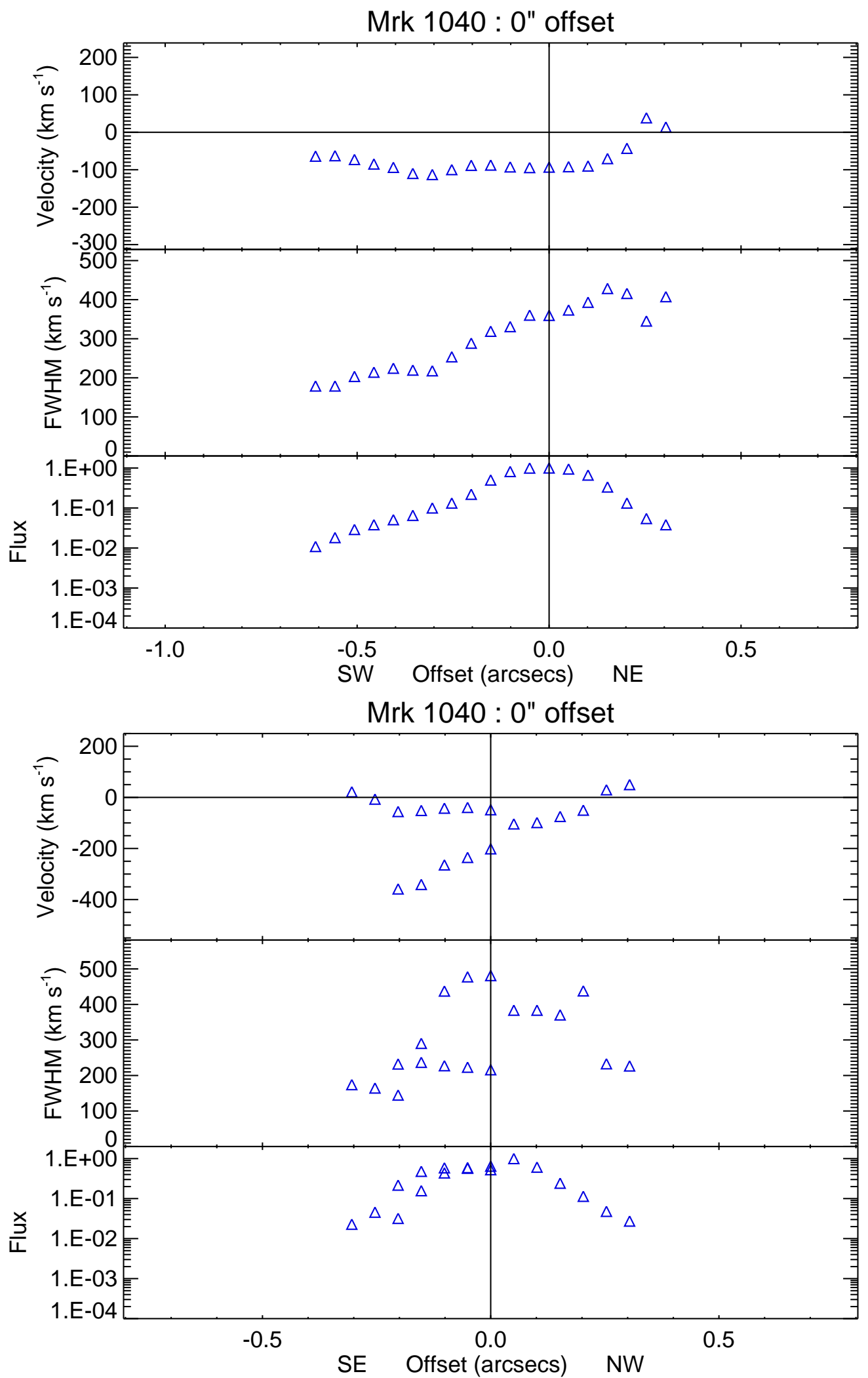

Figure C.13 Top: STIS slit P.A. = -144.20, Bottom: STIS slit P.A. = 119.65 

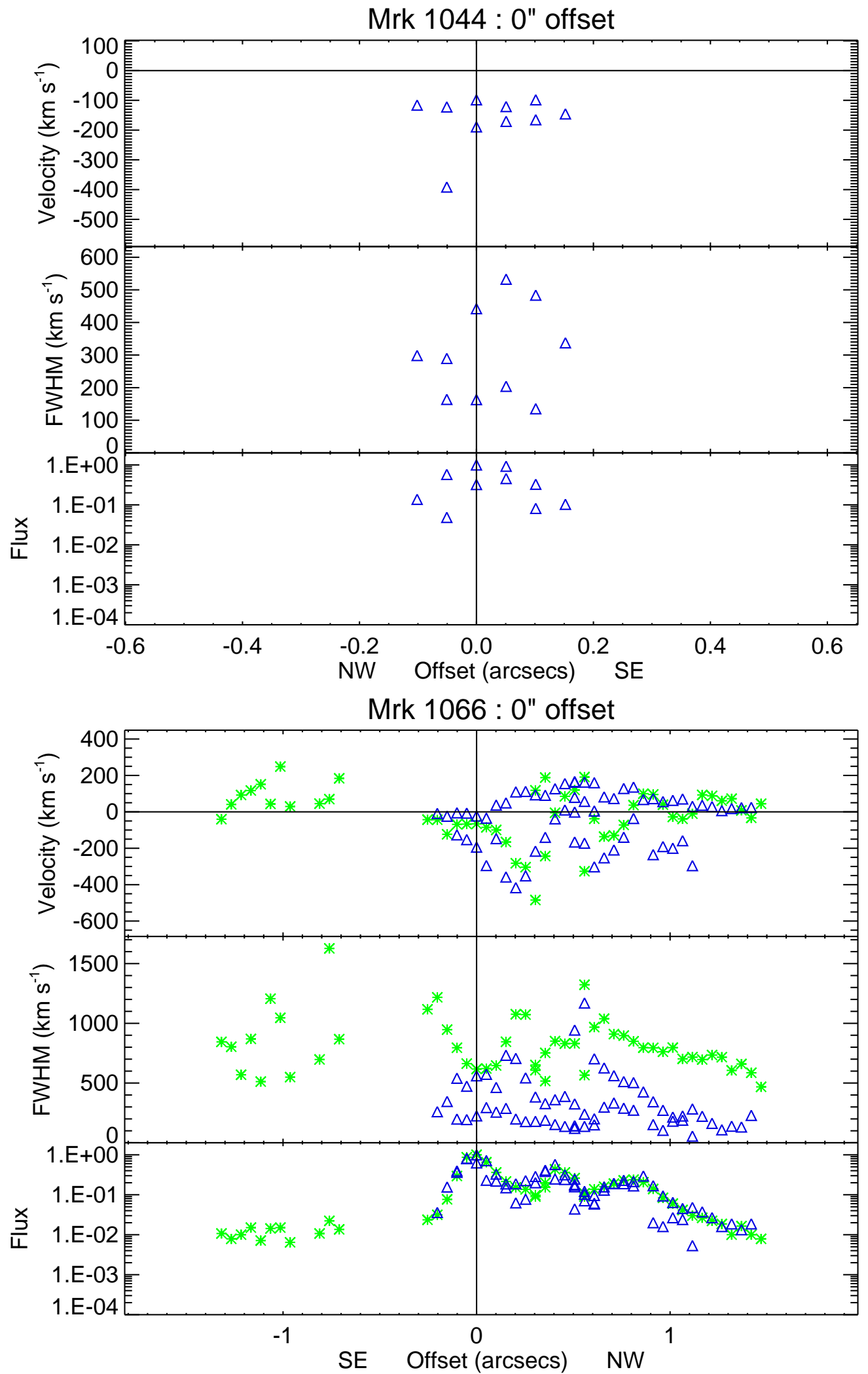

Figure C.14 

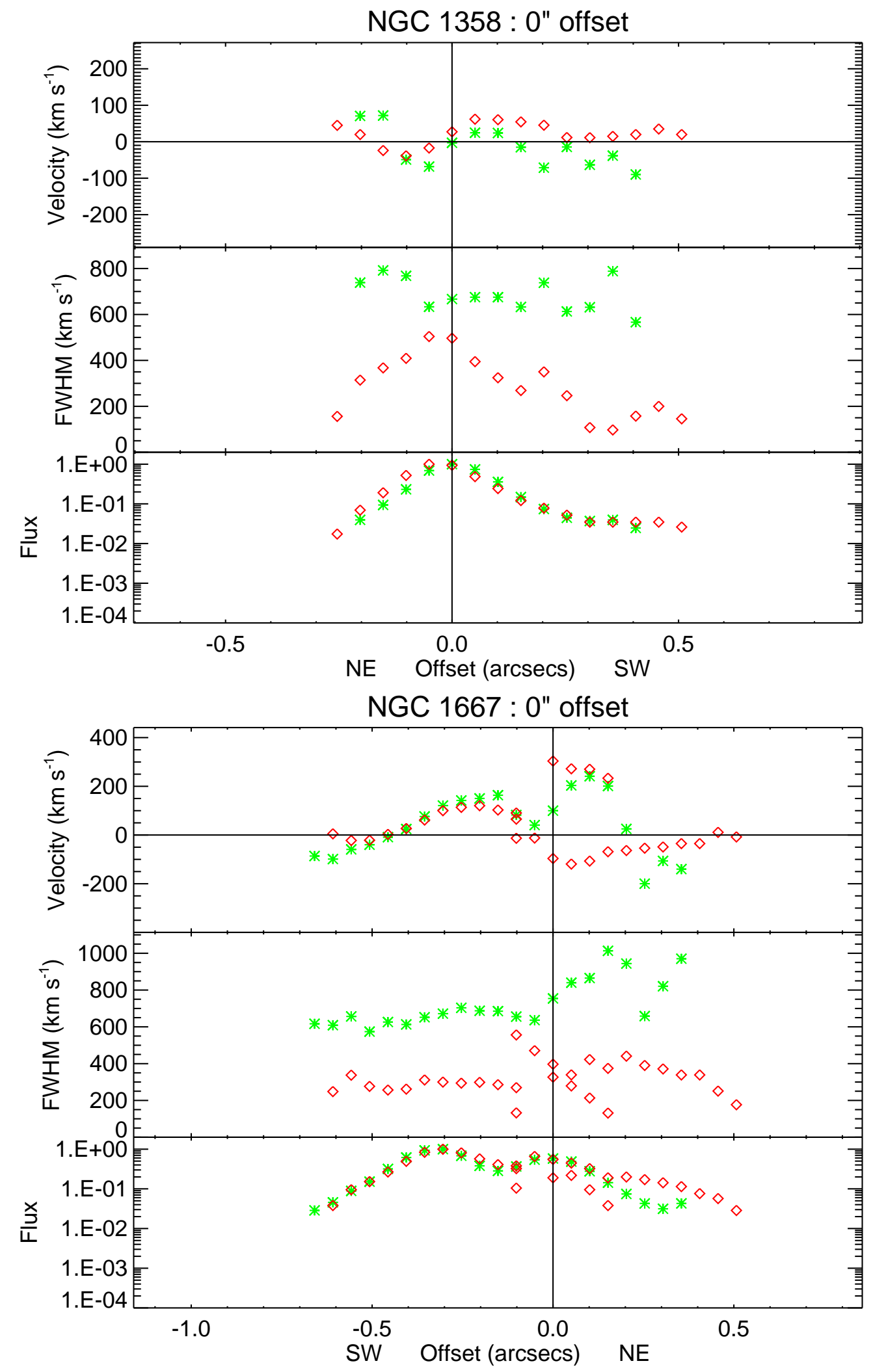

Figure C.15 

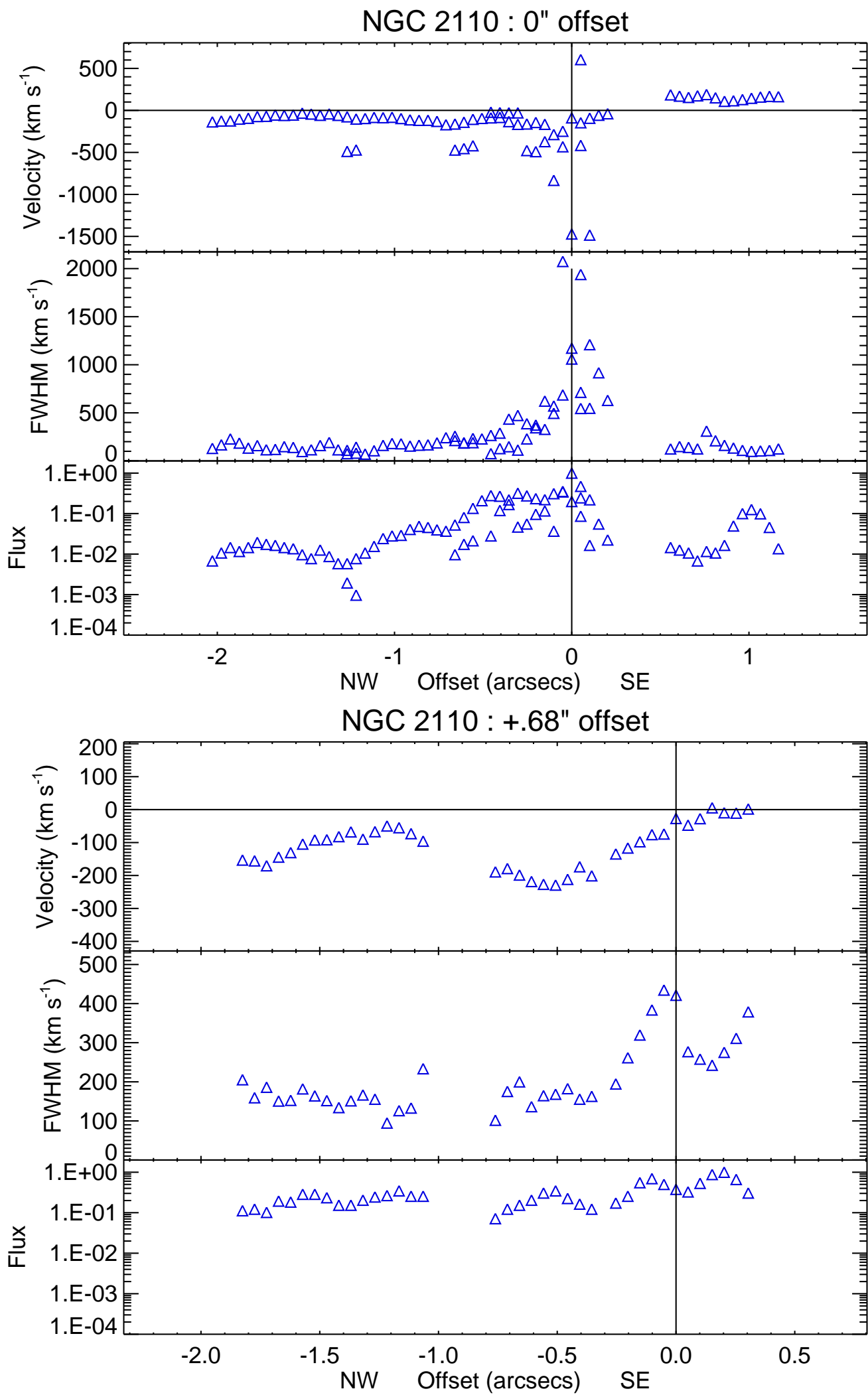

Figure C.16 

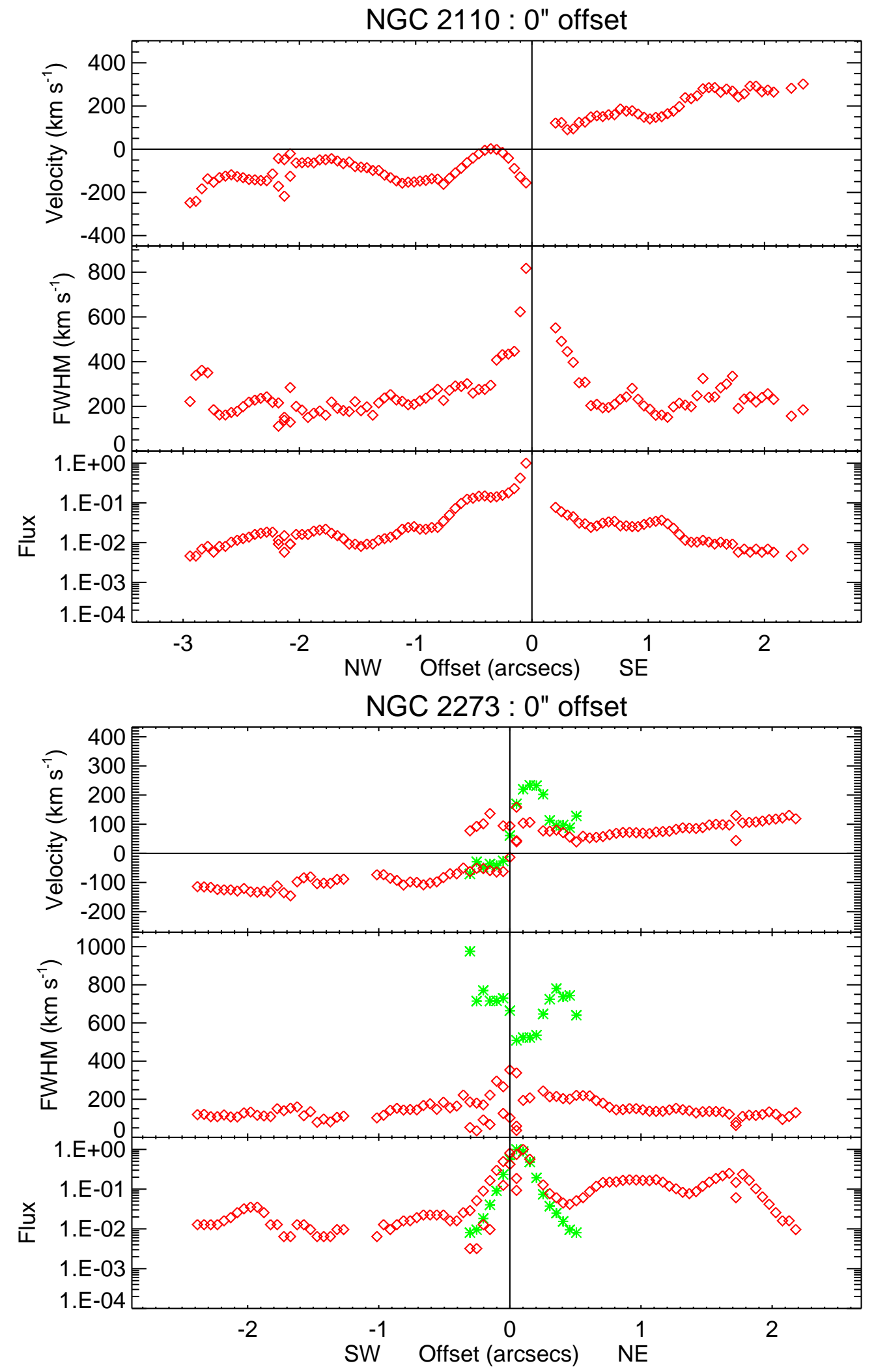

Figure C.17 

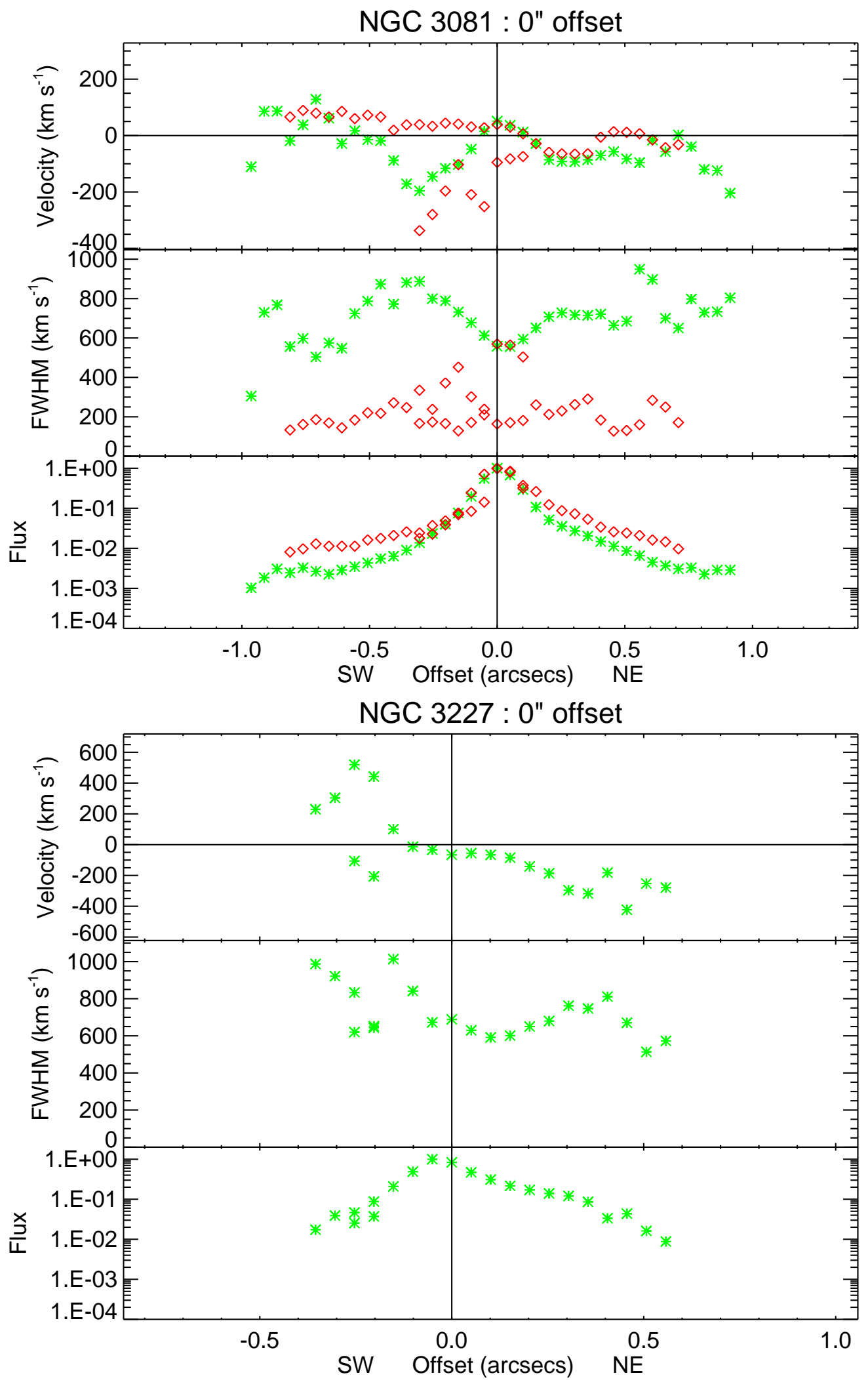

Figure C.18 

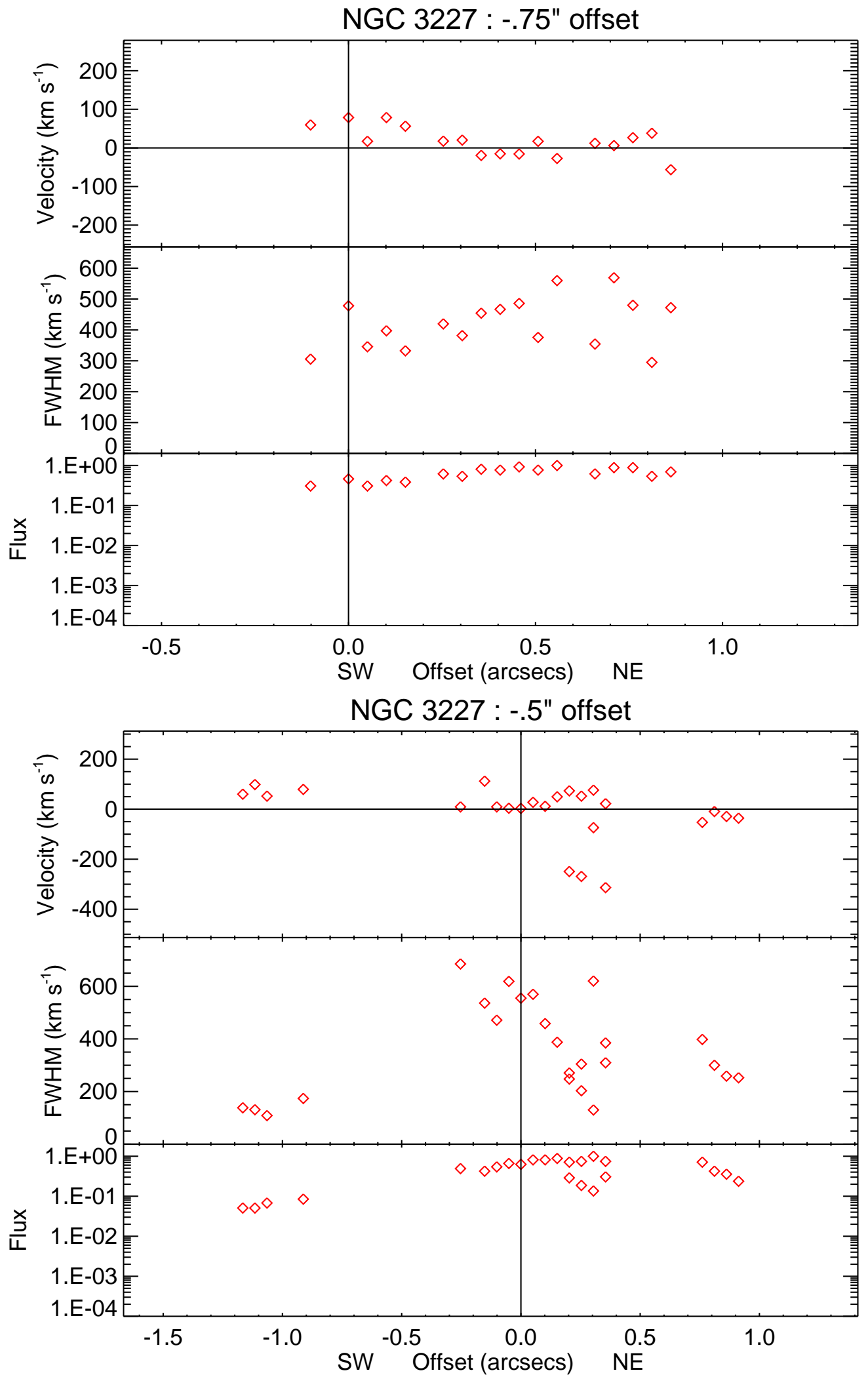

Figure C.19 

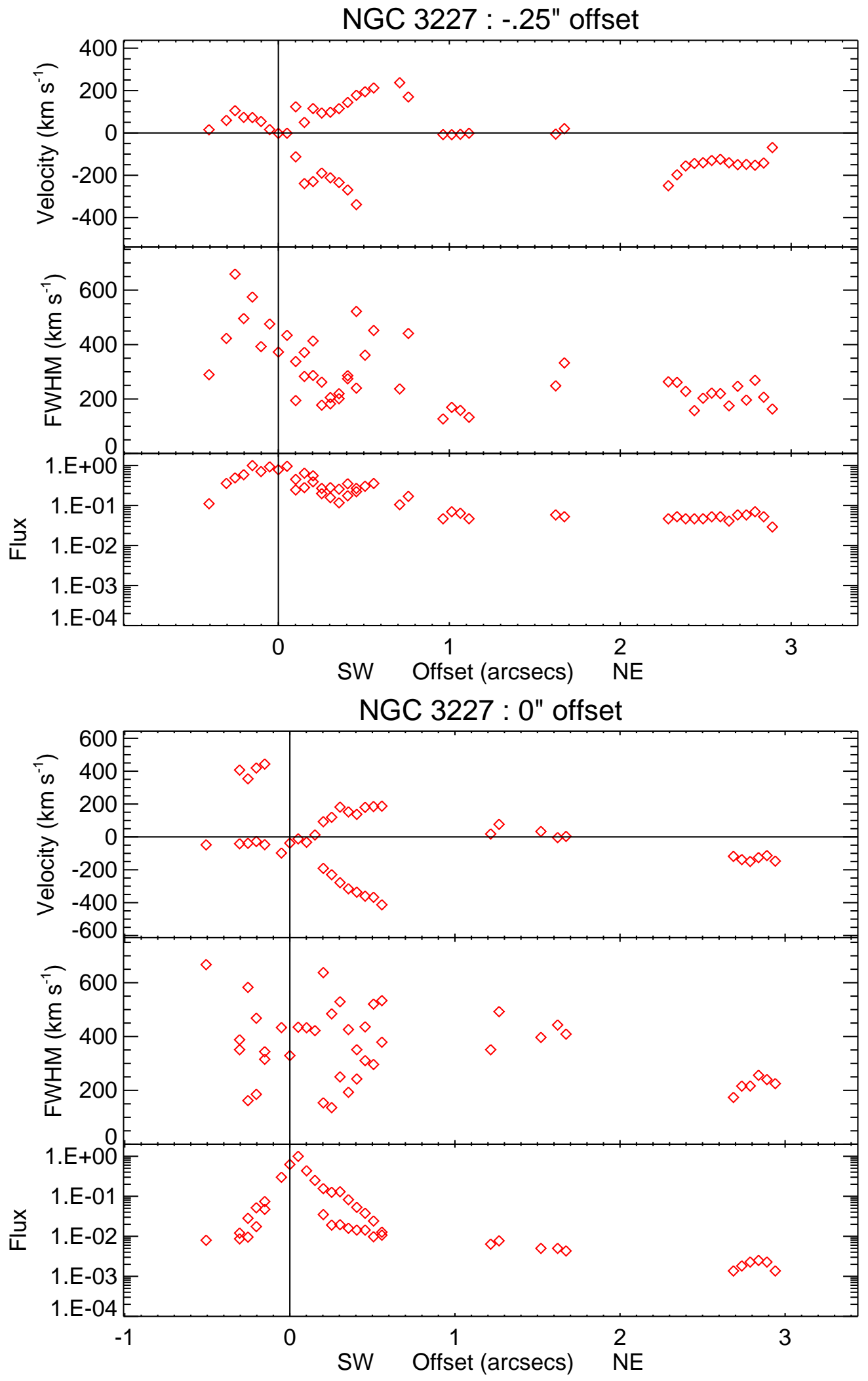

Figure C.20 

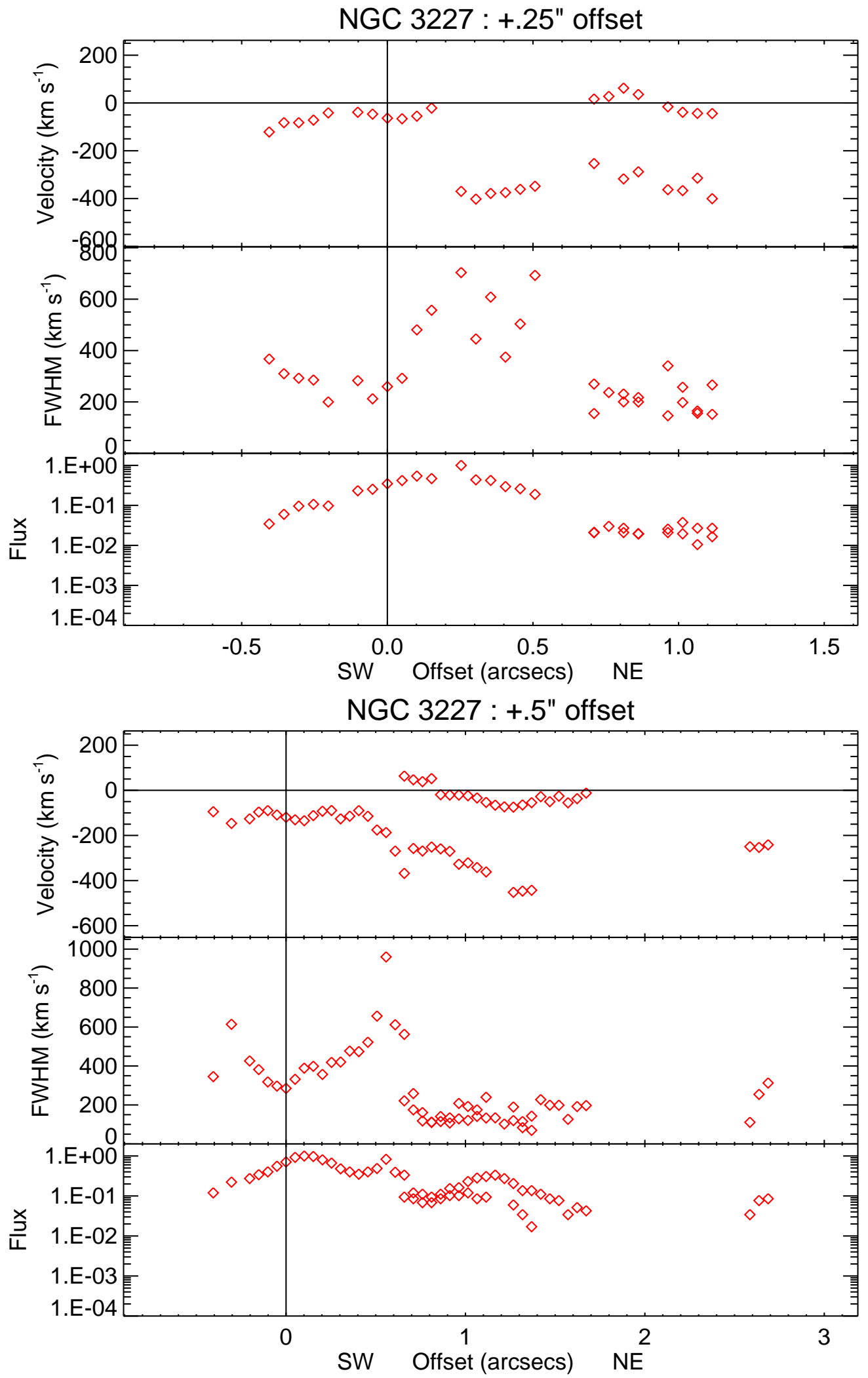

Figure C.21 

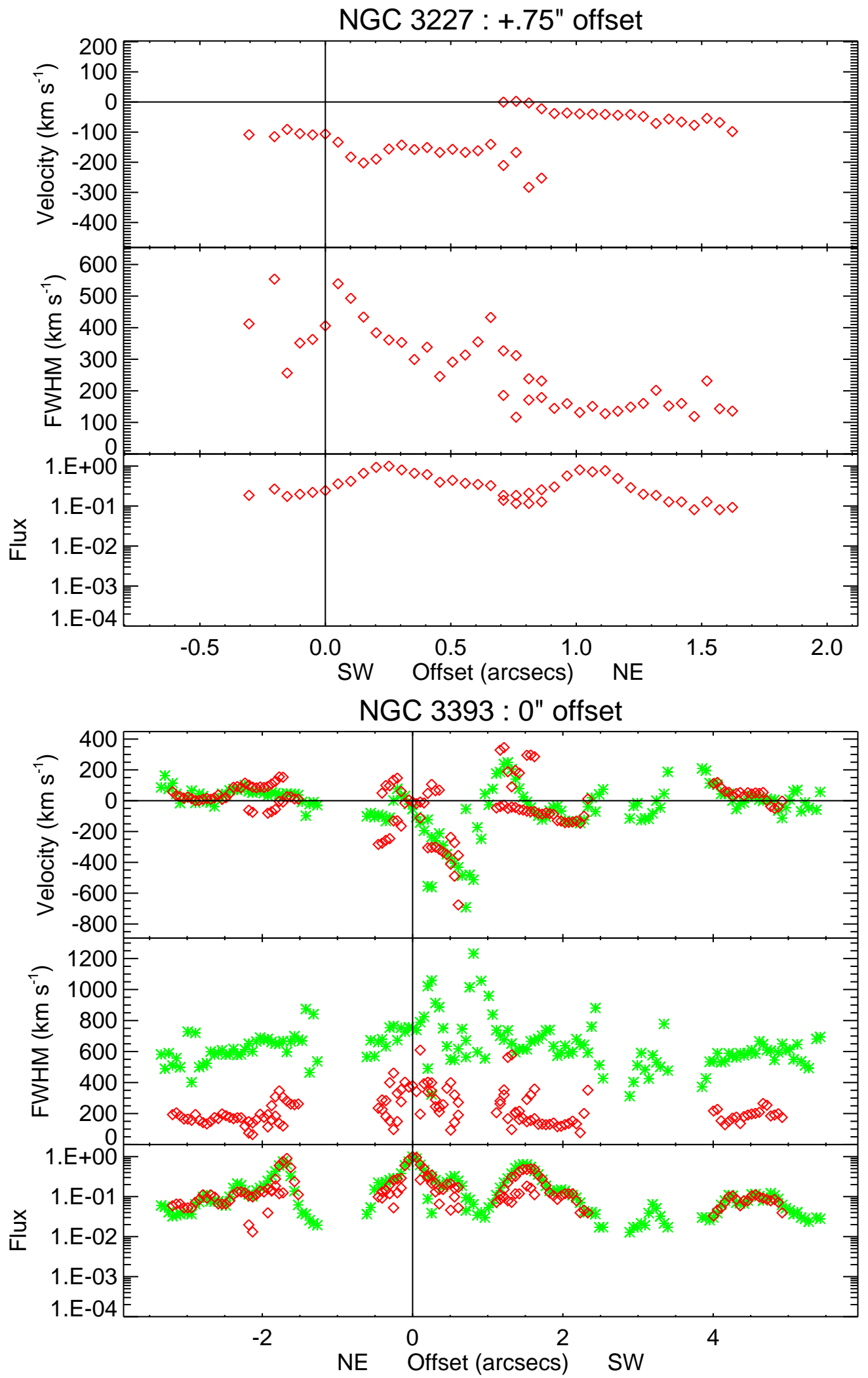

Figure C.22 

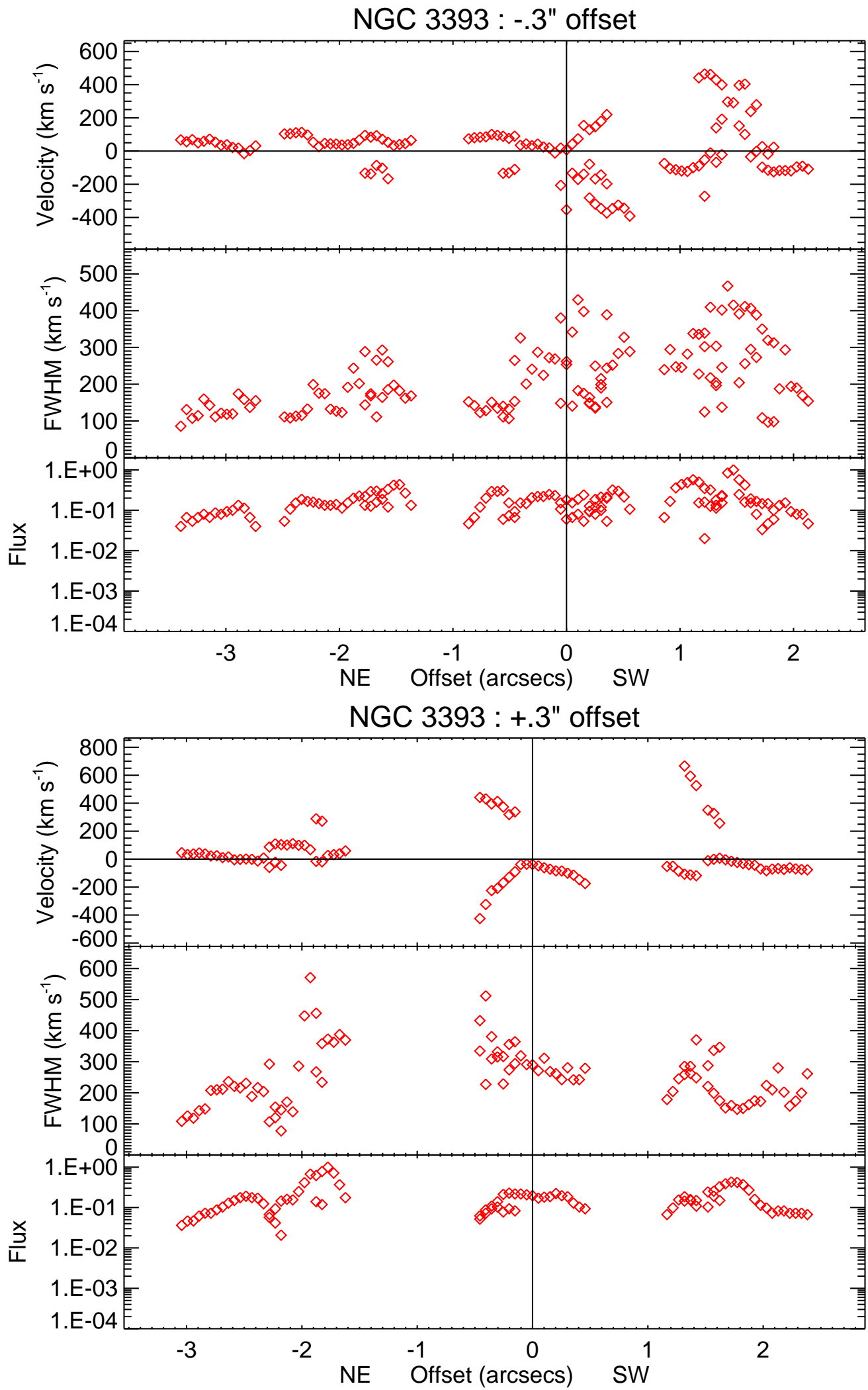

Figure C.23 
NGC $3783: 0 "$ offset

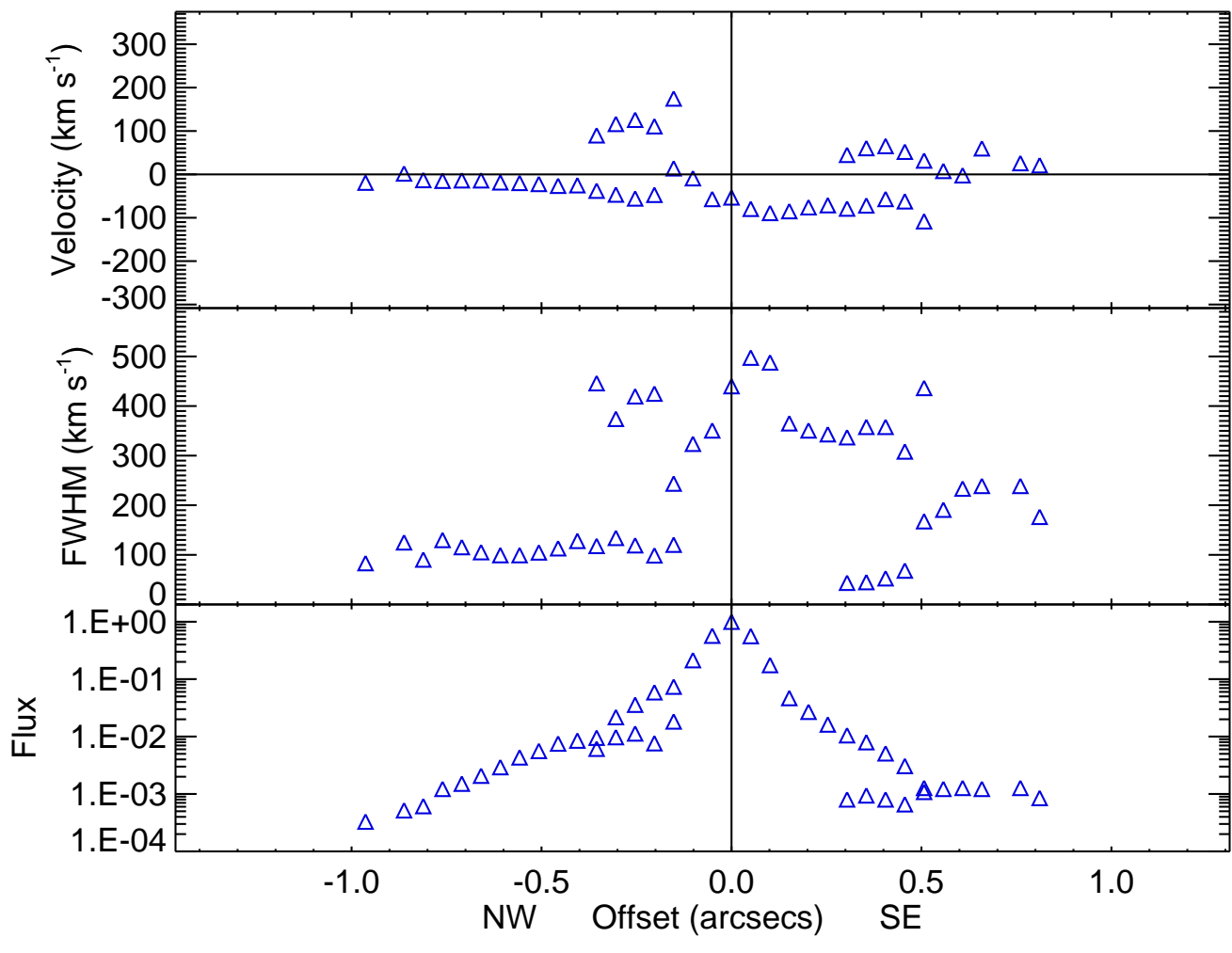

NGC 4051: -.05" offset

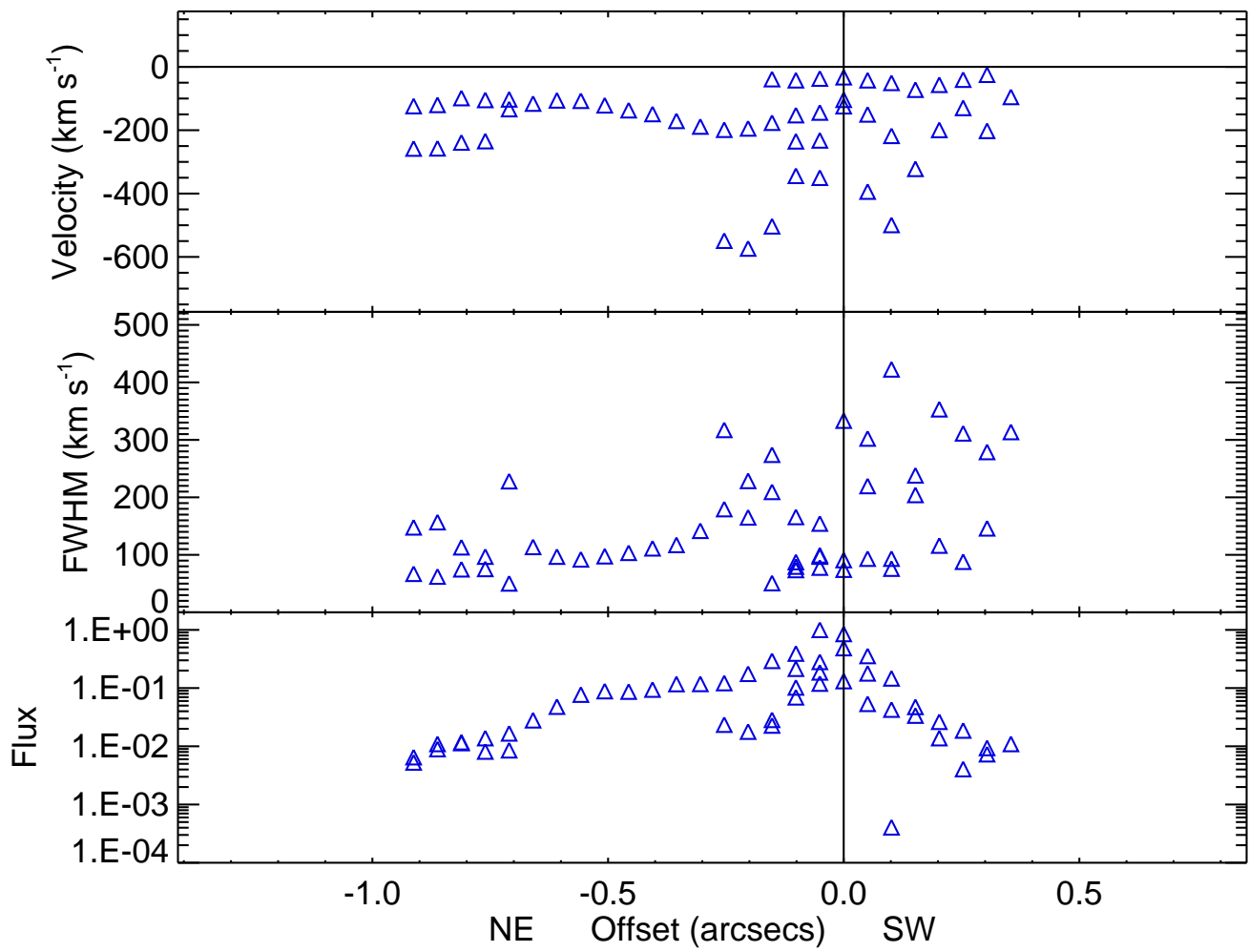

Figure C.24 

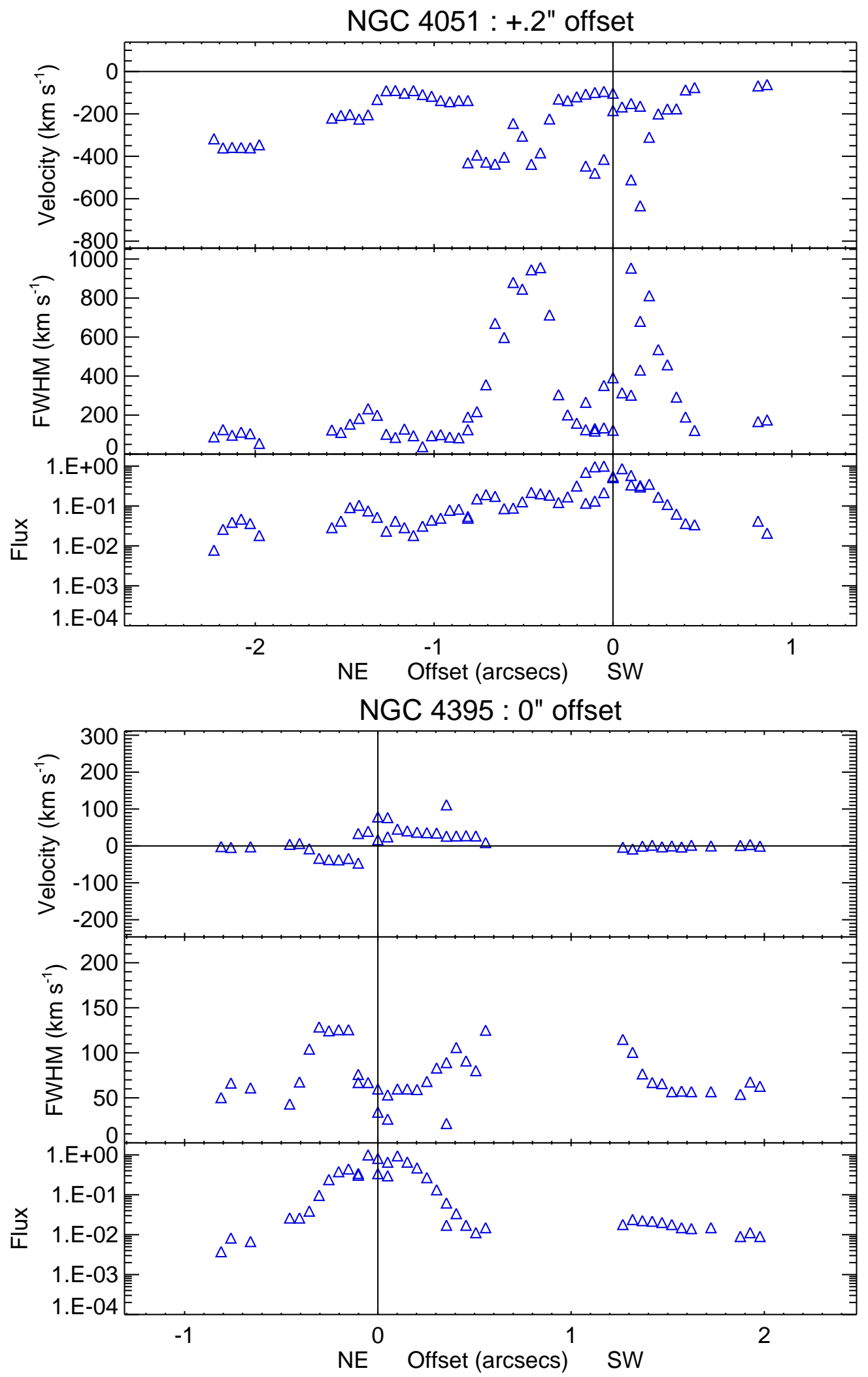

Figure C.25 

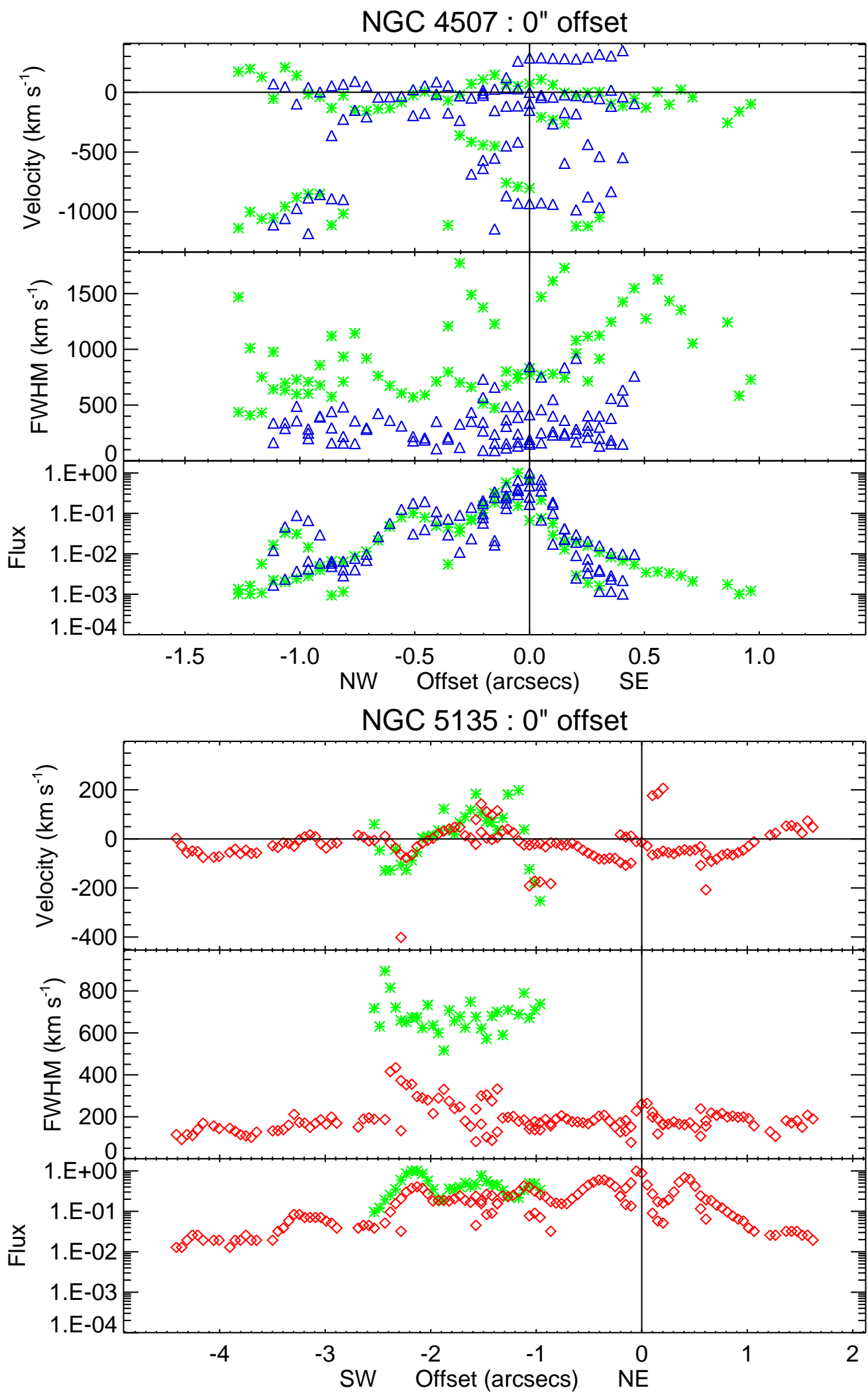

Figure C.26 

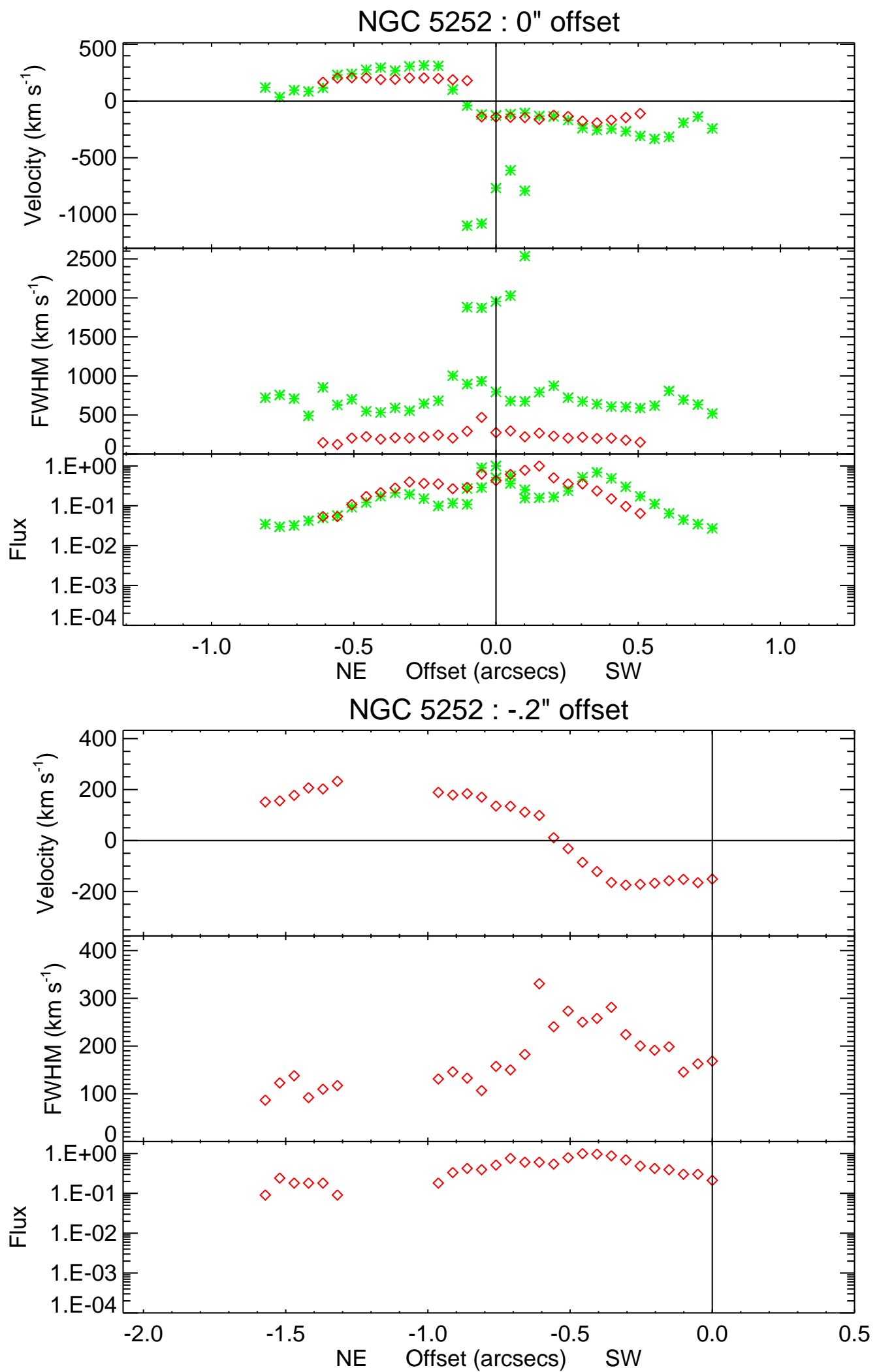

Figure C.27 

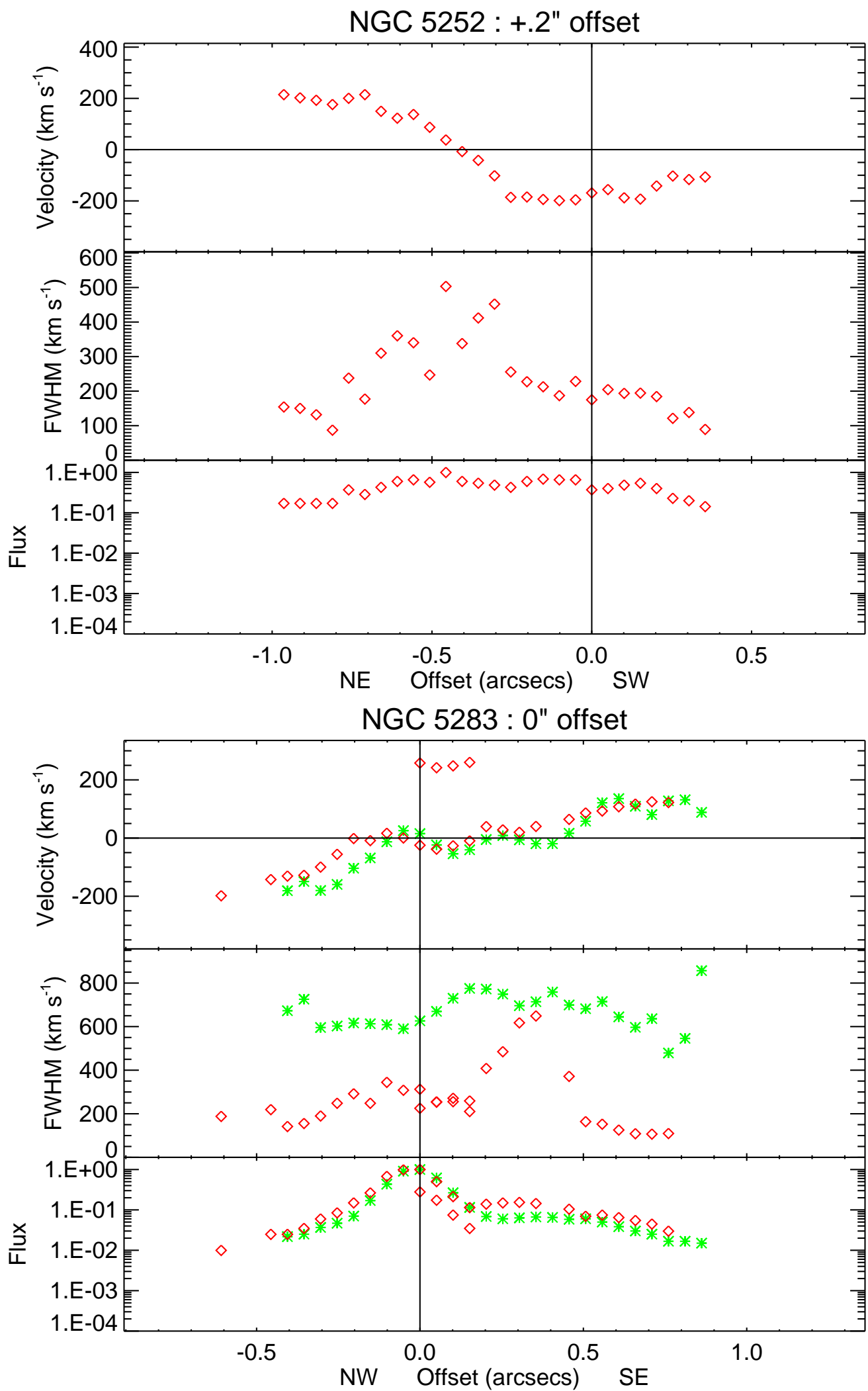

Figure C.28 


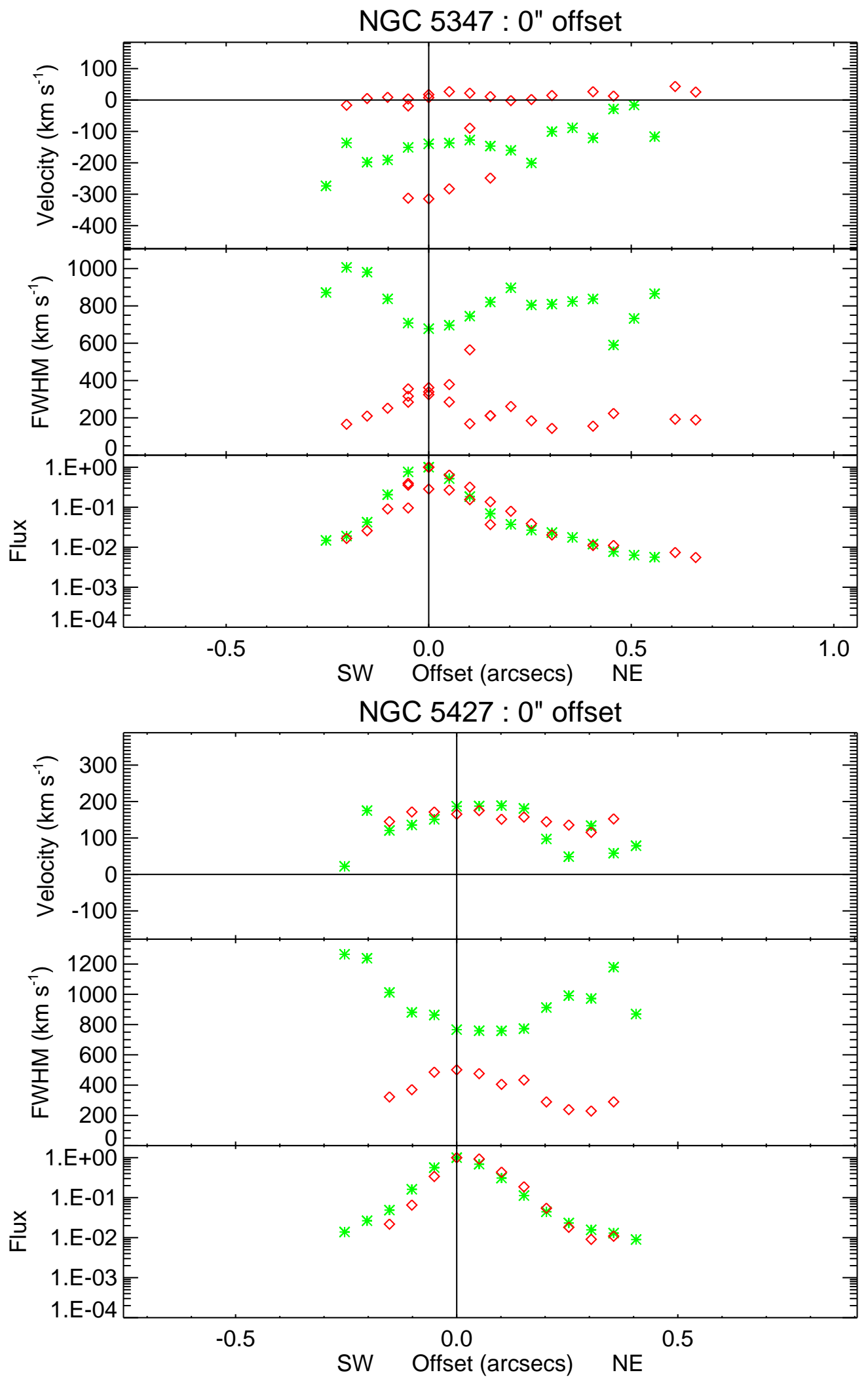

Figure C.29 

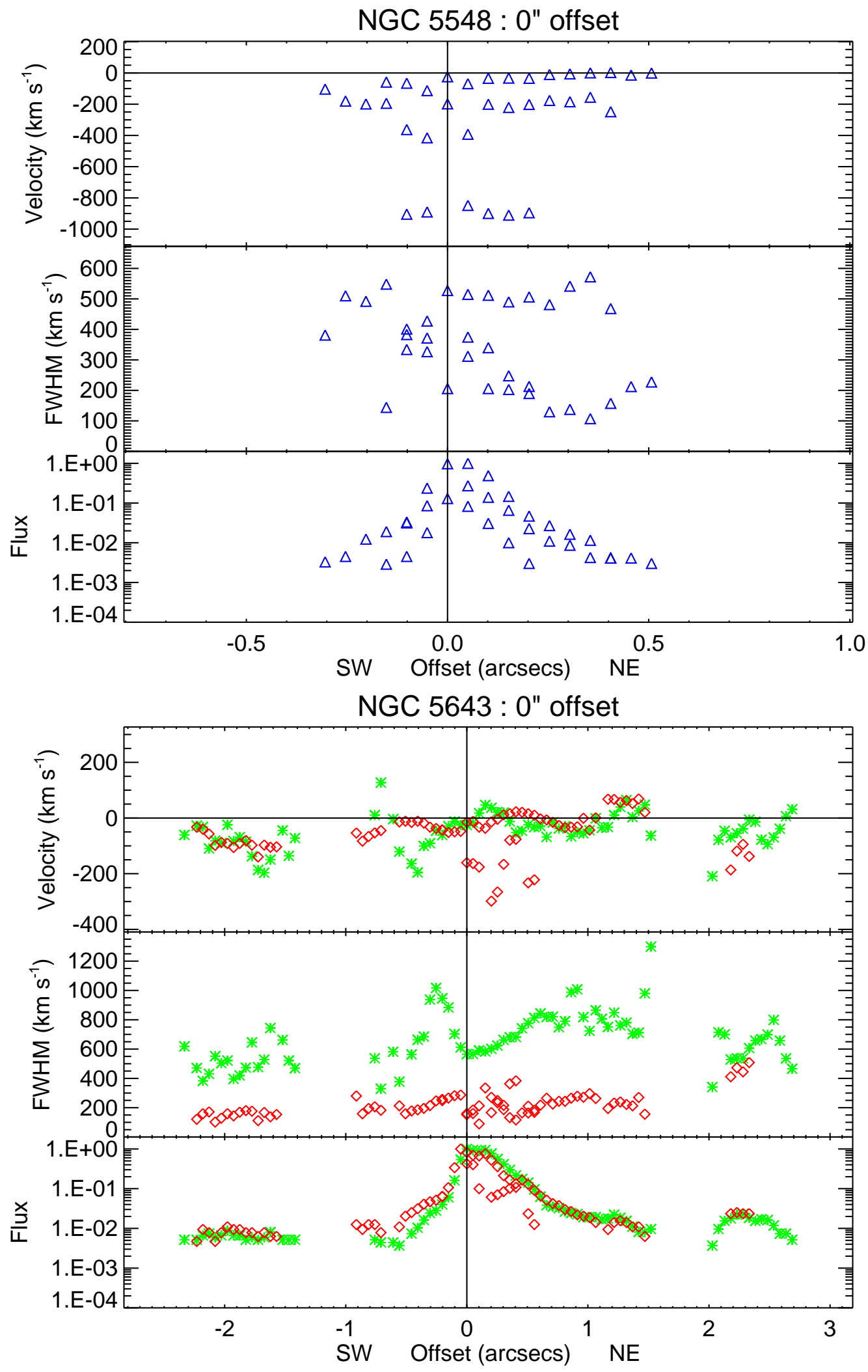

Figure C.30 

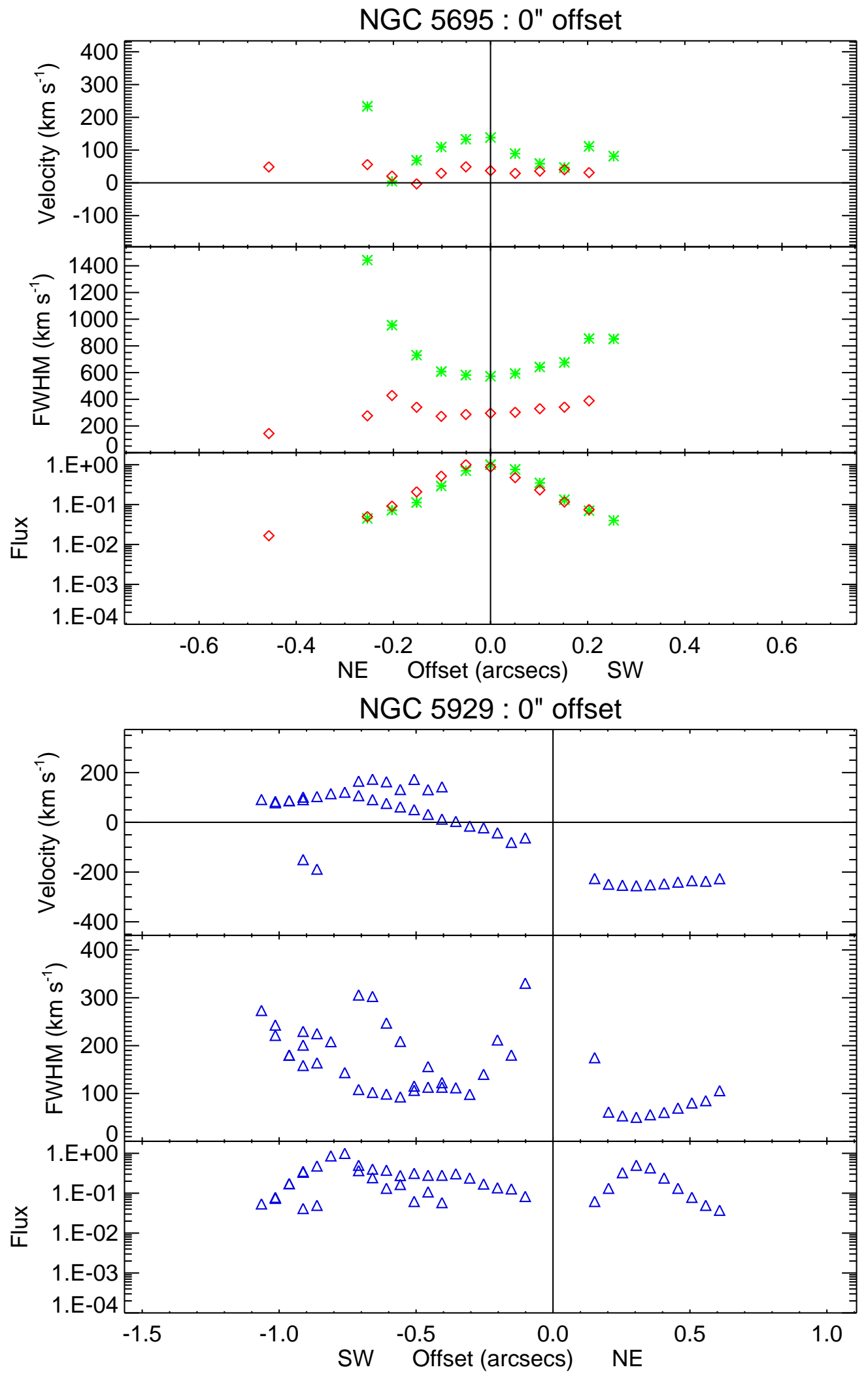

Figure C.31 

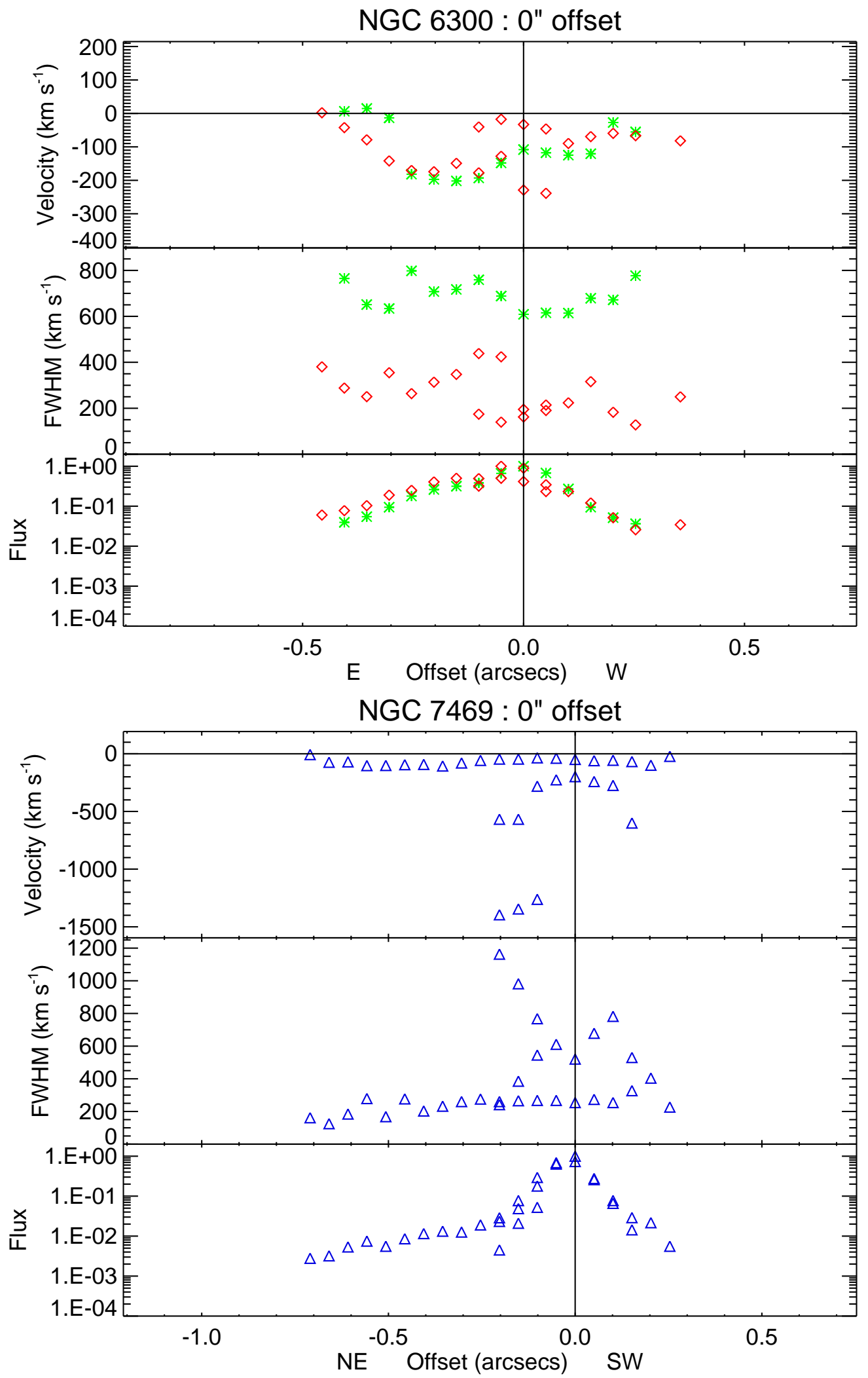

Figure C.32 
NGC 7674 : 0" offset

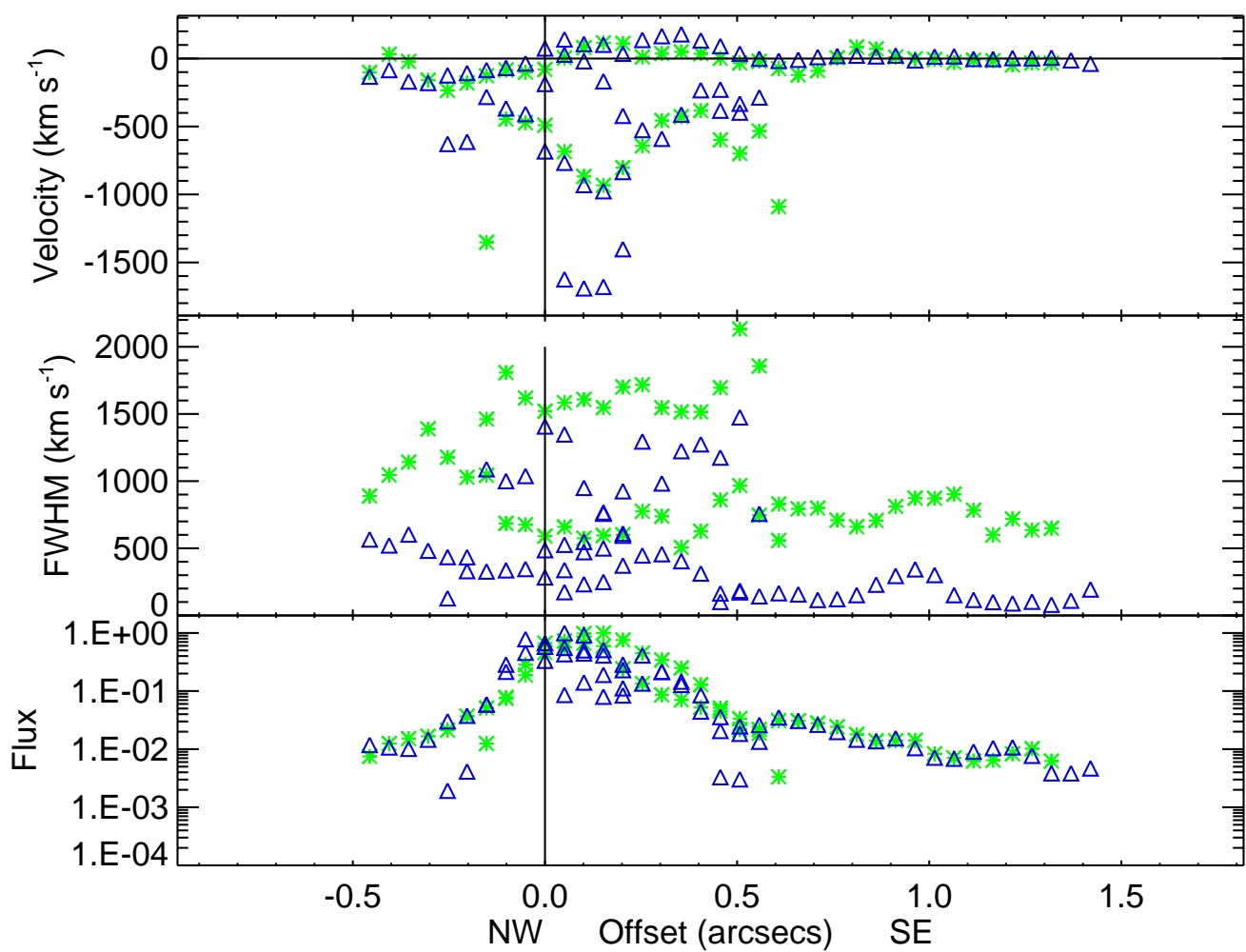

NGC 7682 : 0" offset

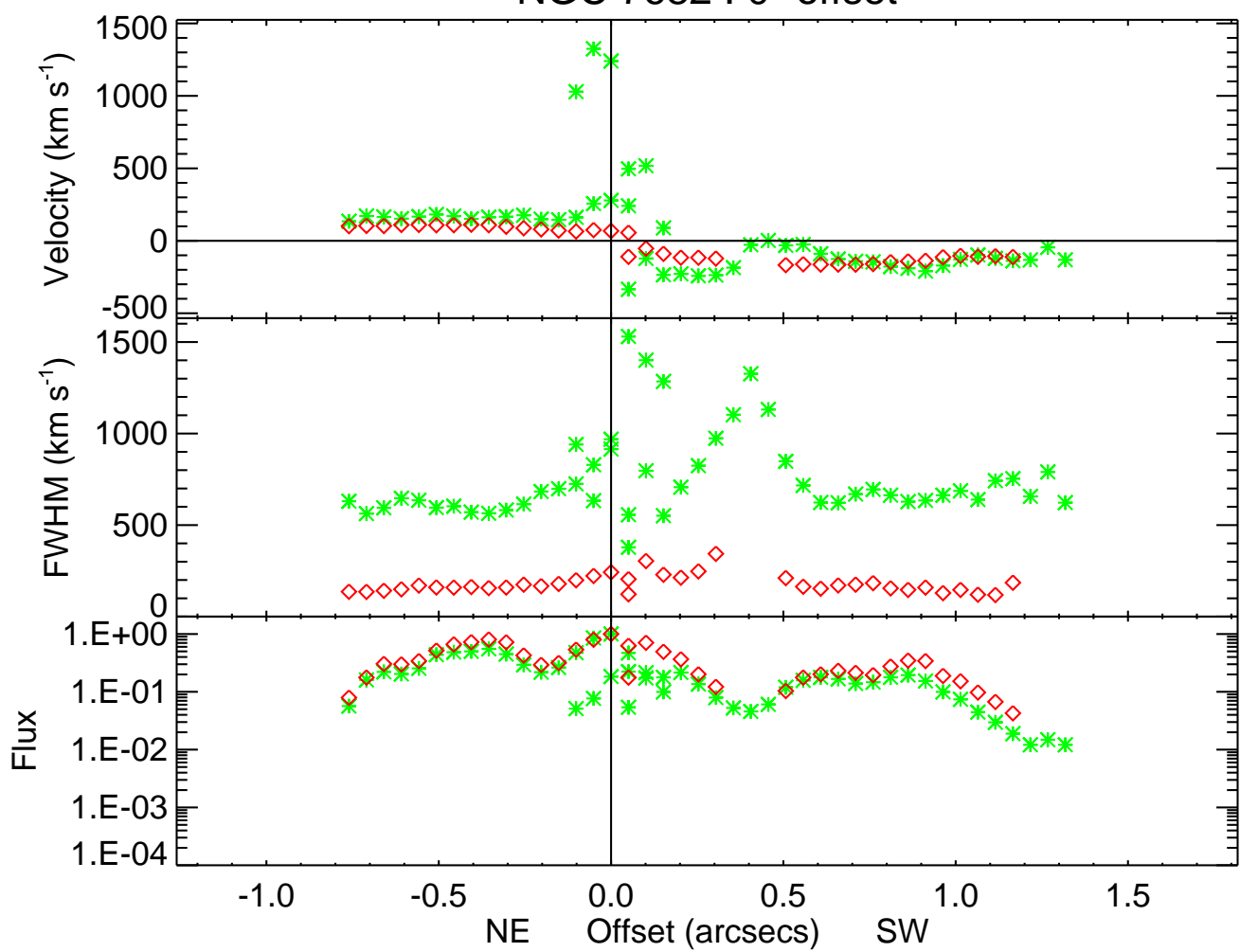

Figure C.33 

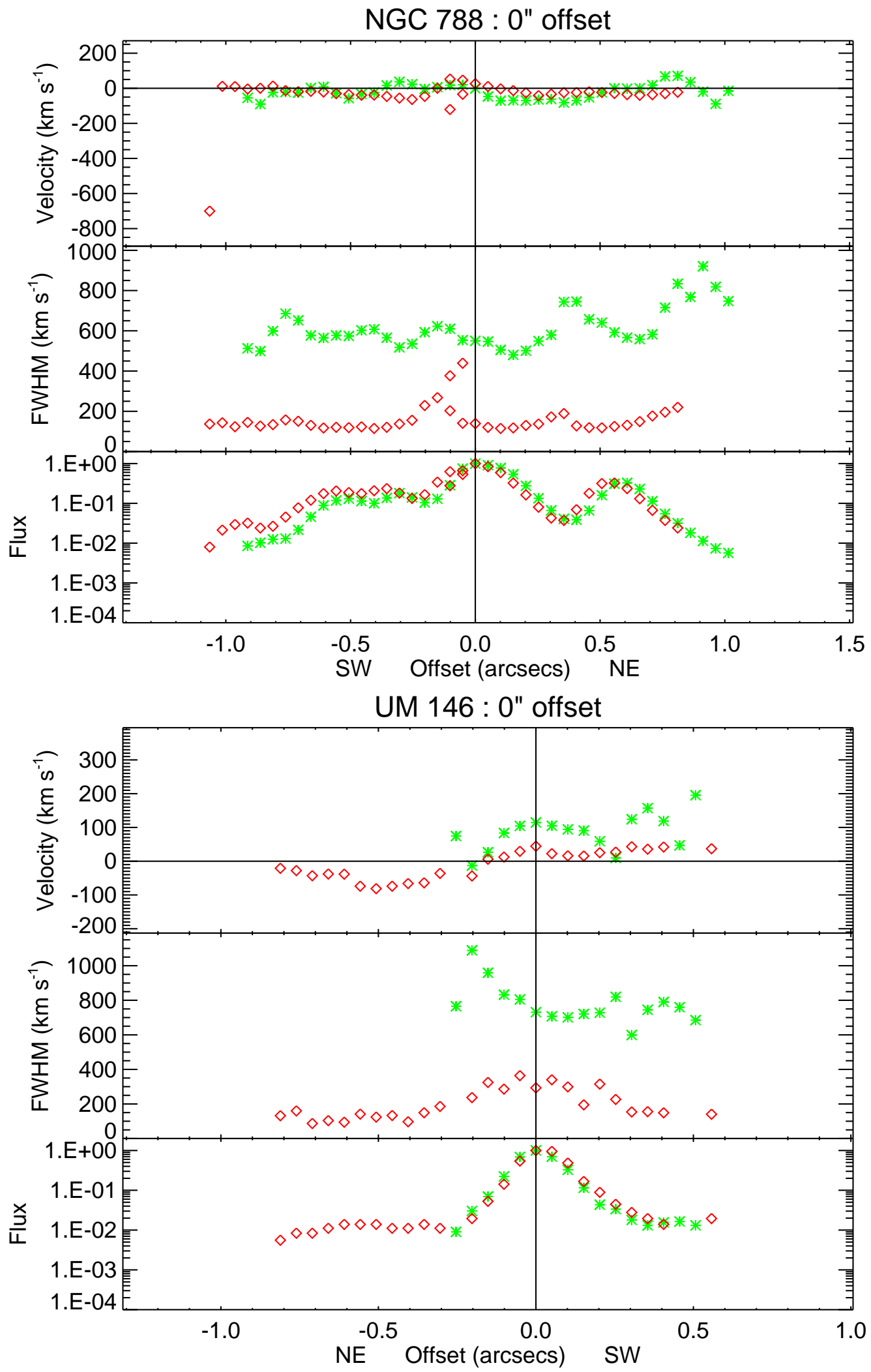

Figure C.34 


\section{APPENDIX D}

\section{Extended Sample: AGN Kinematic Models}

Kinematics model chosen as the best fit for our radial velocity data set for modeled AGN. Parameters used to create this model are given in Table 6.4. 

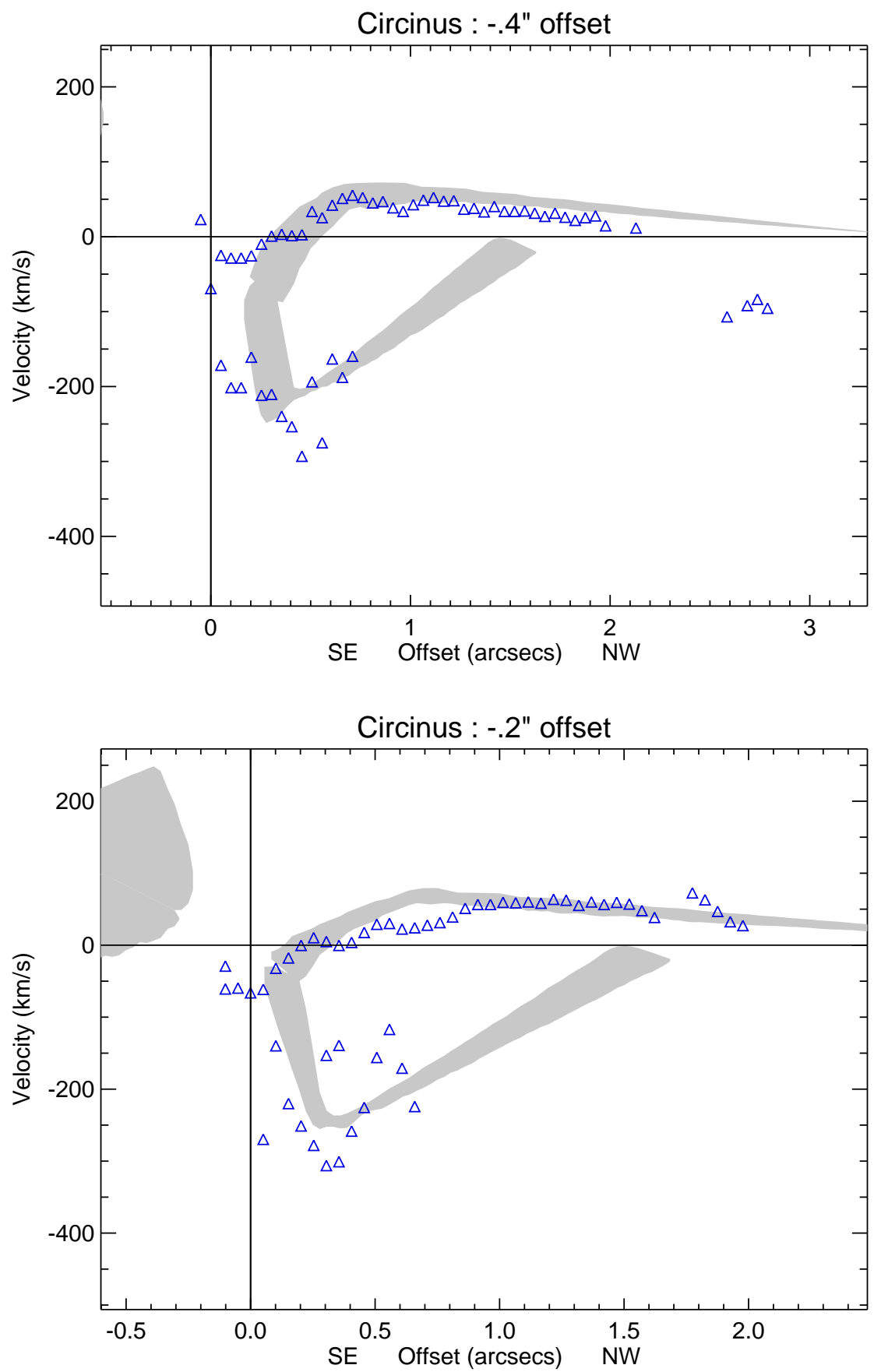

Figure D.1 

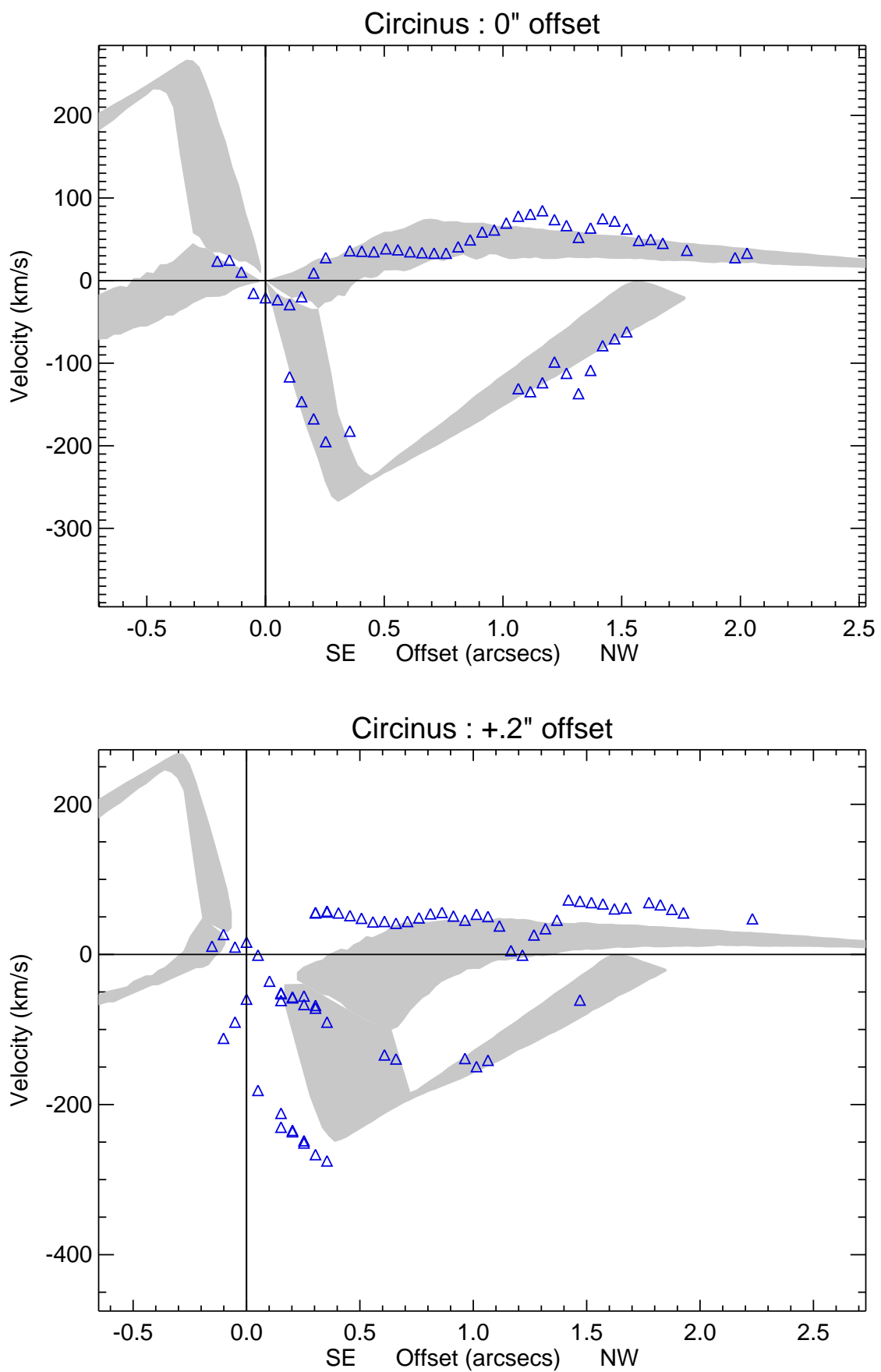

Figure D.2 

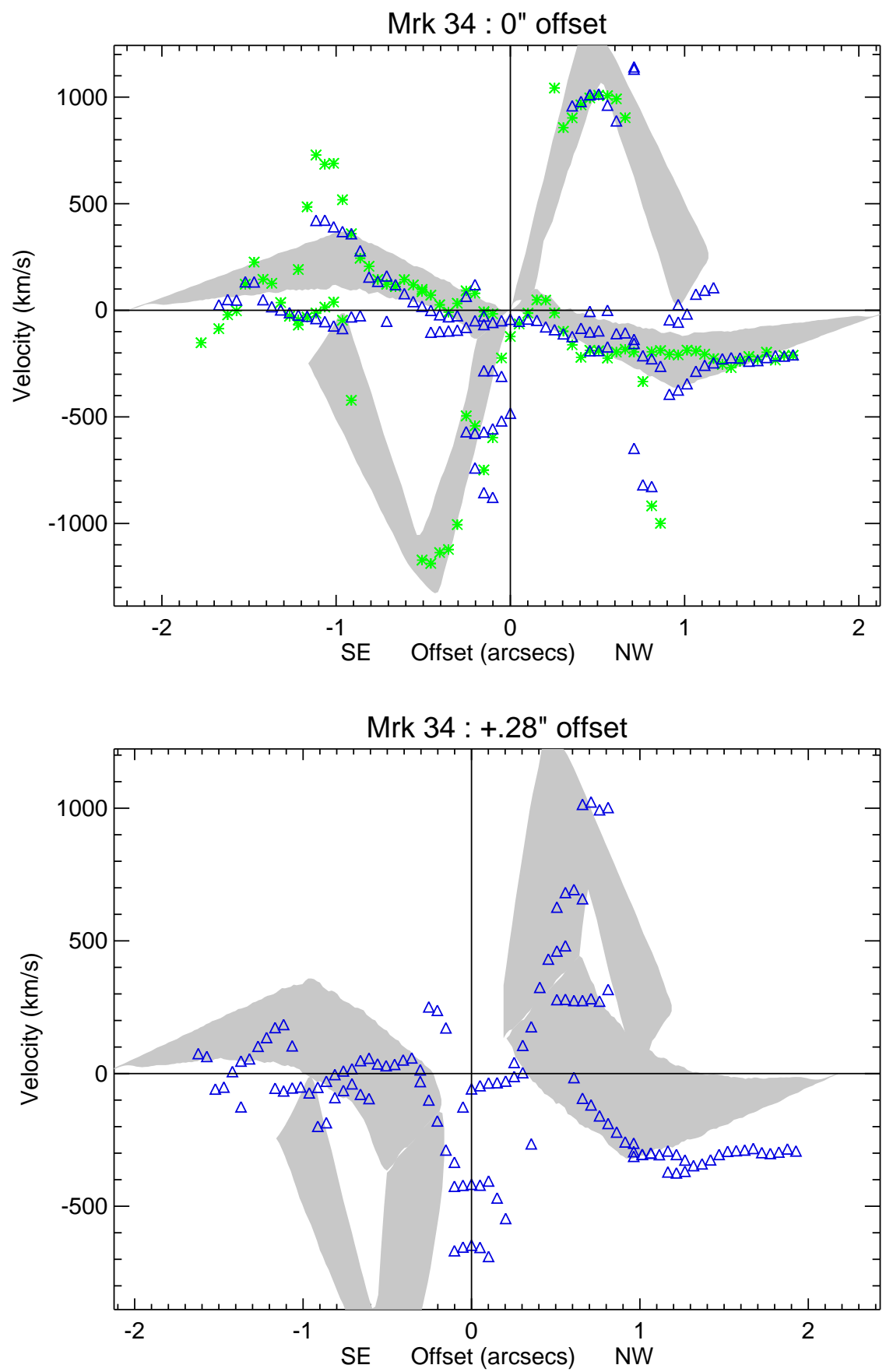

Figure D.3 

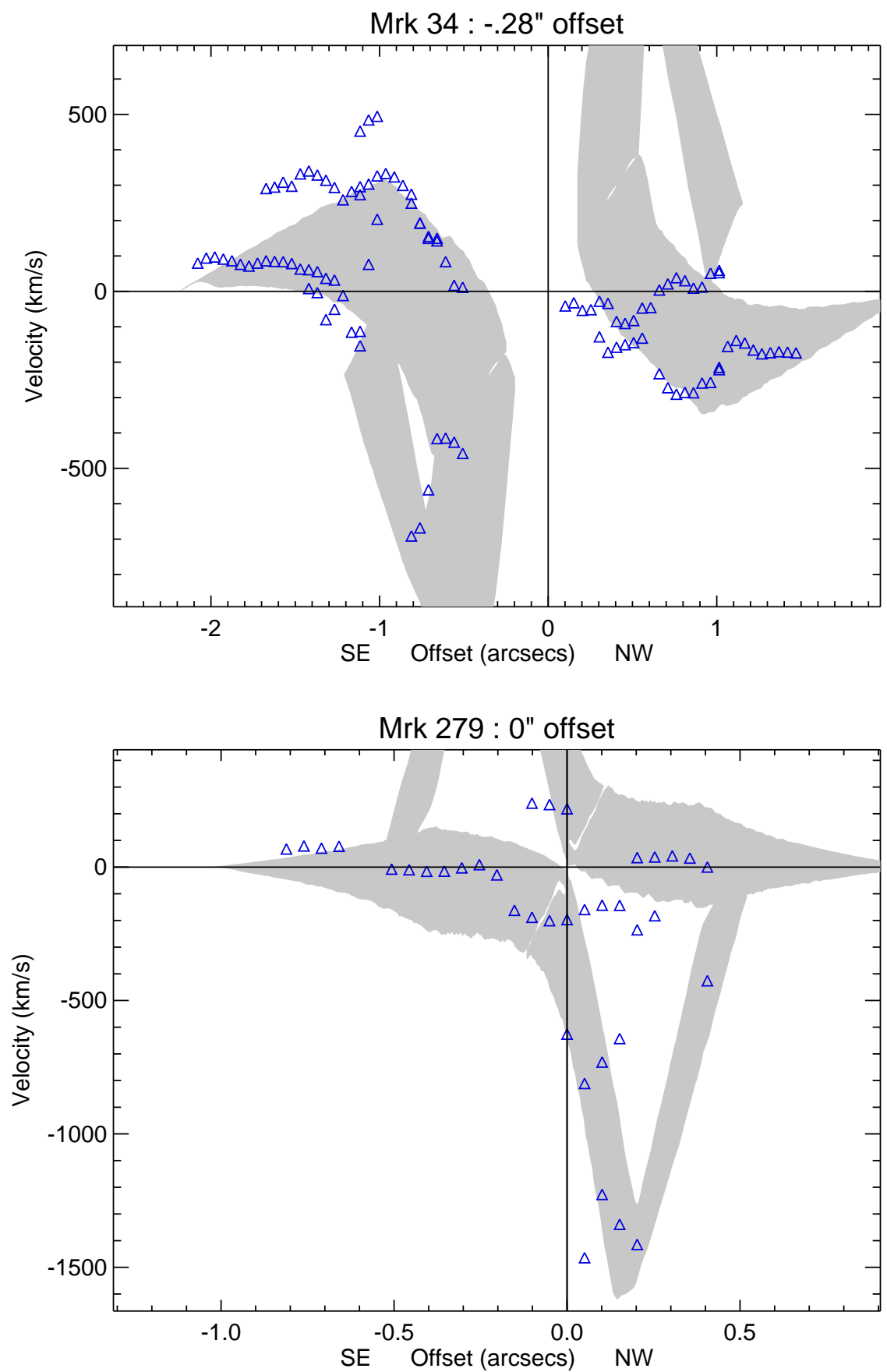

Figure D.4 

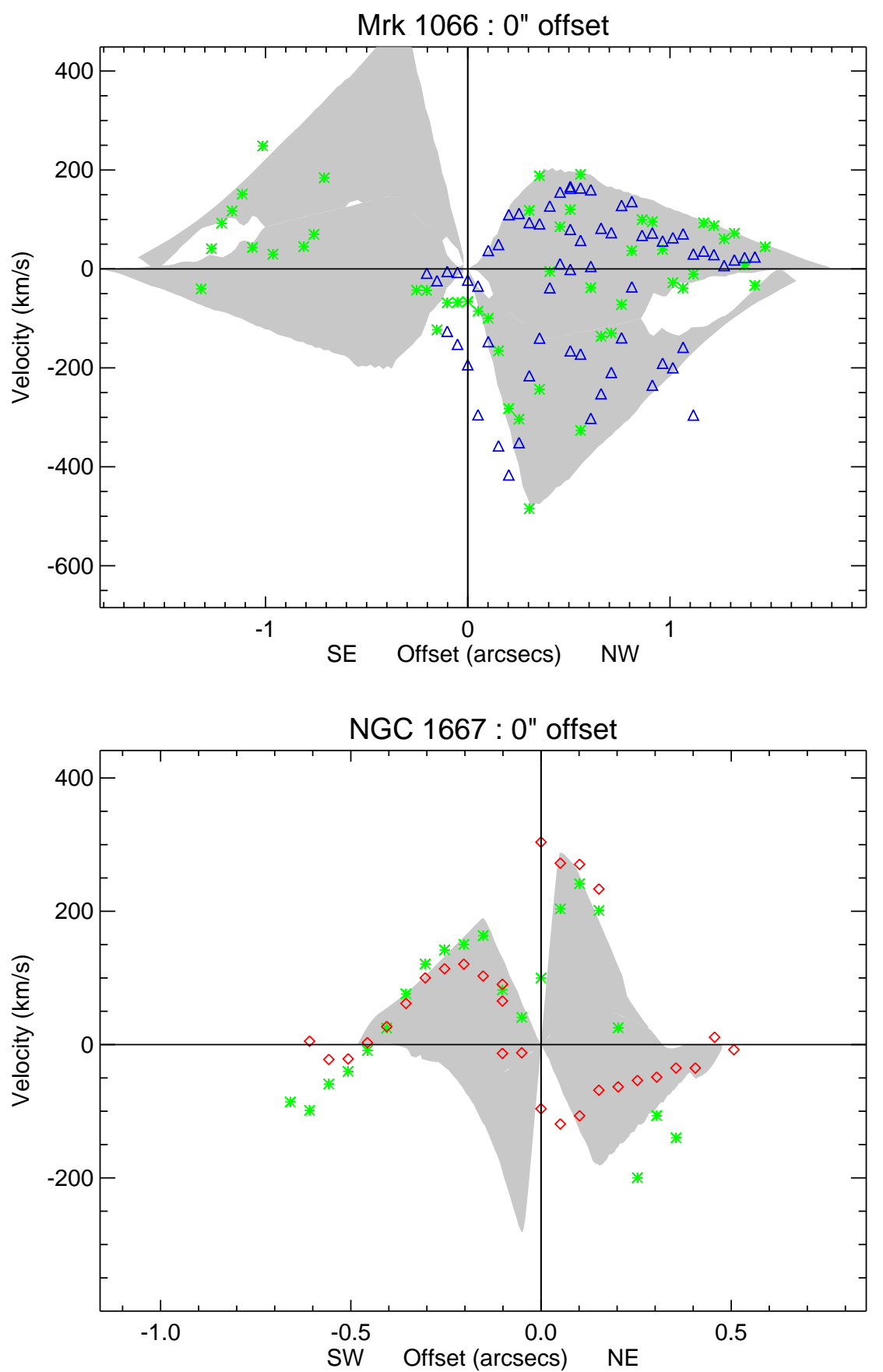

Figure D.5 

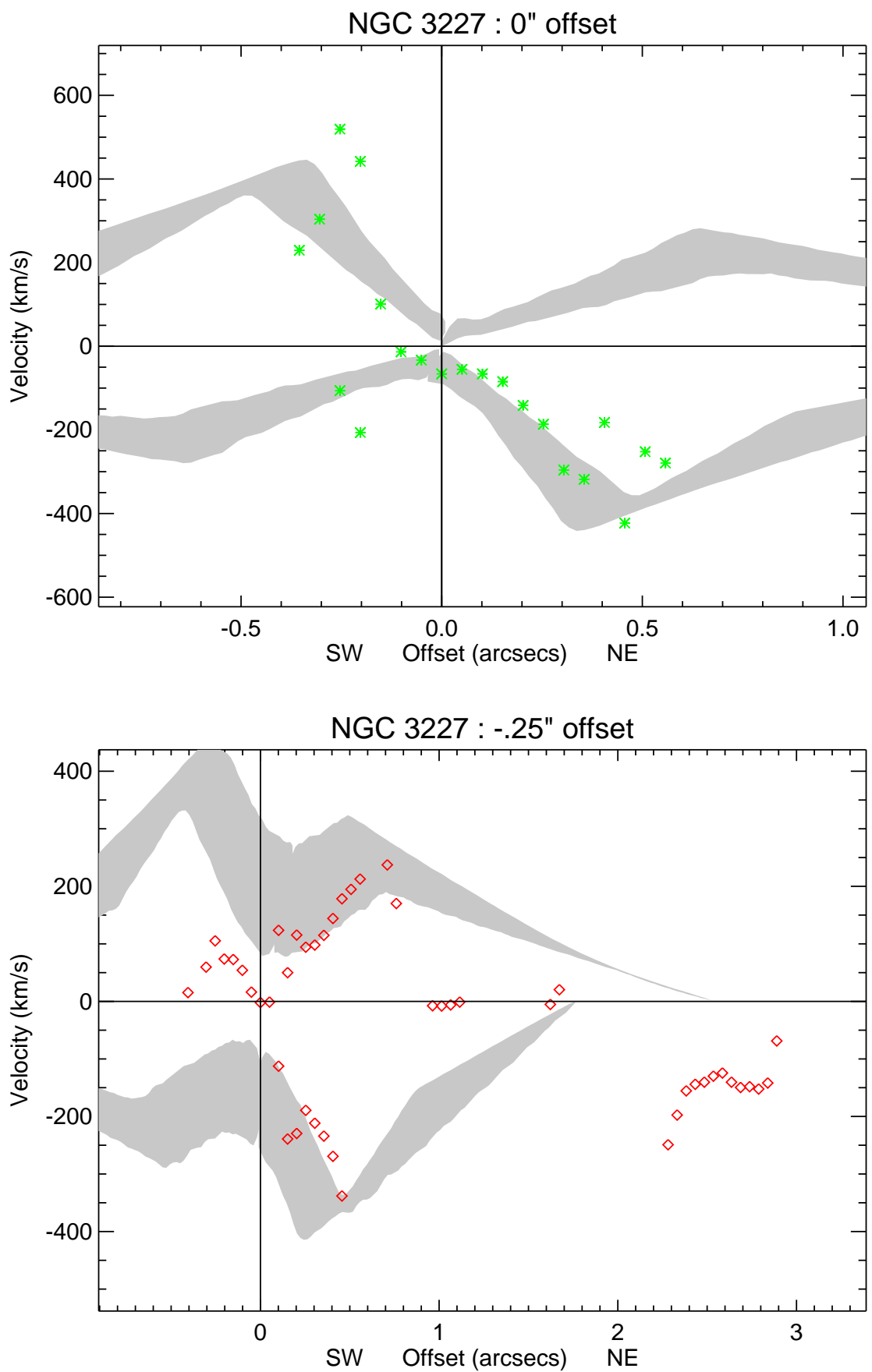

Figure D.6 

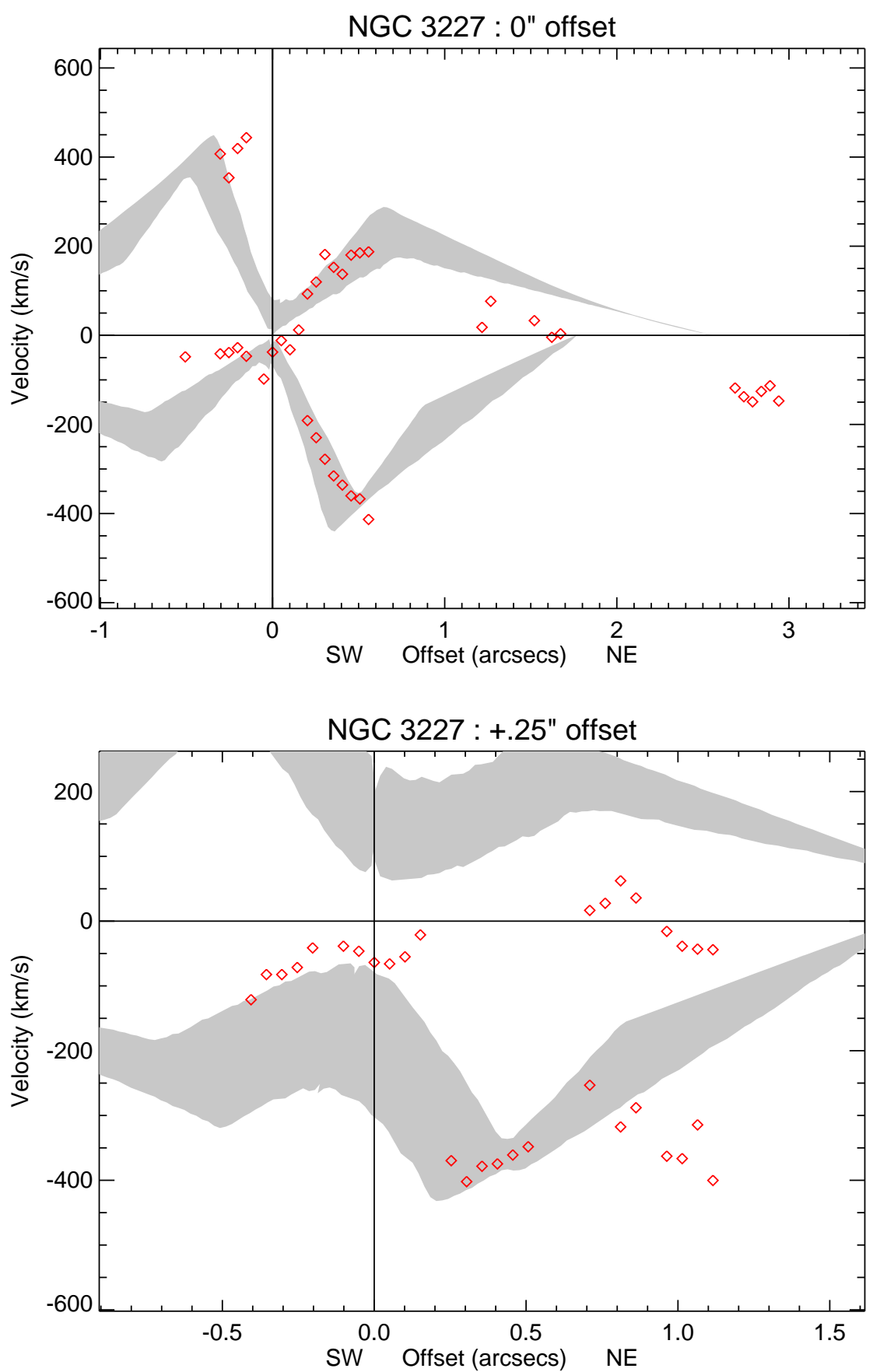

Figure D.7 

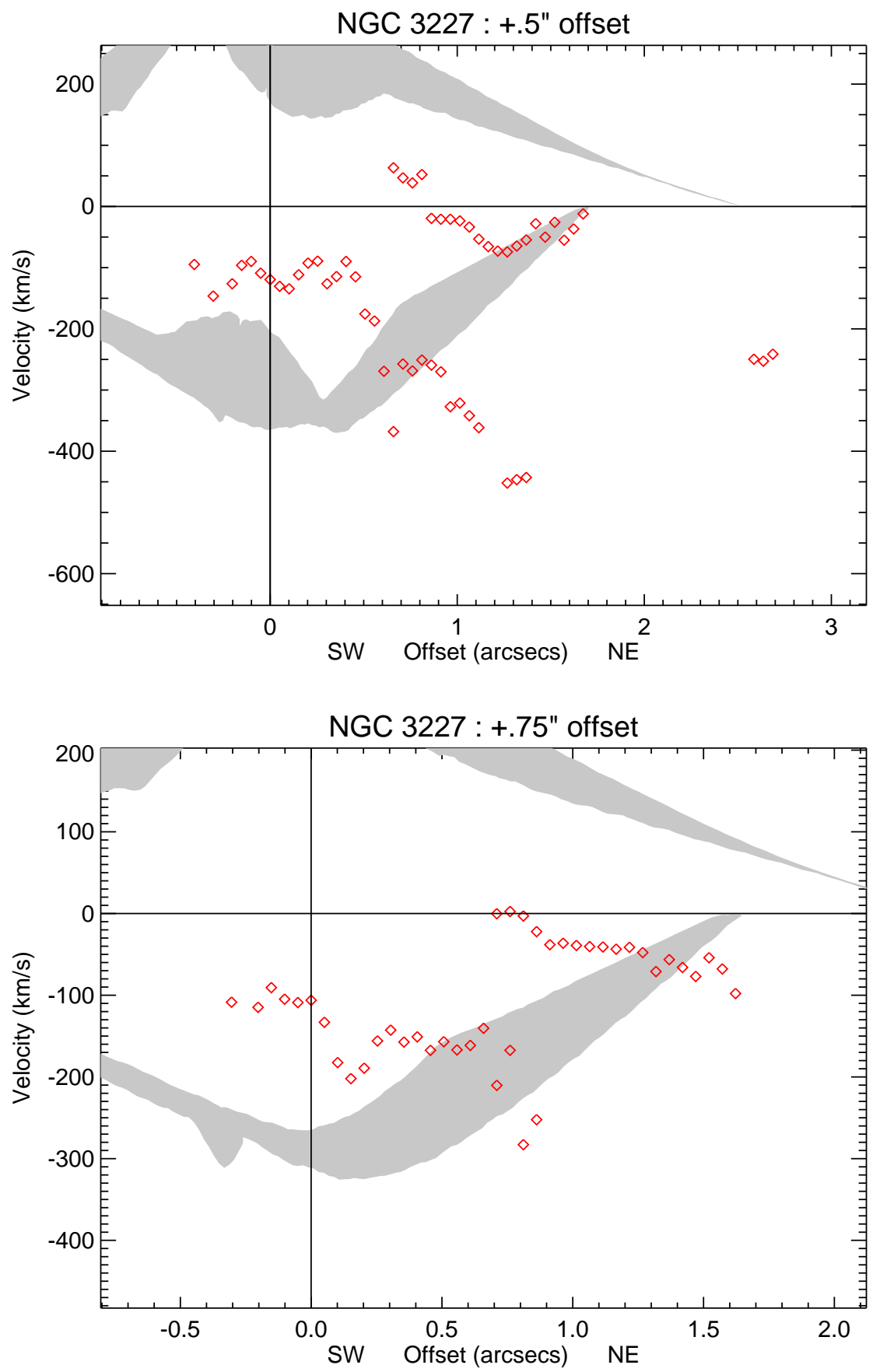

Figure D.8 

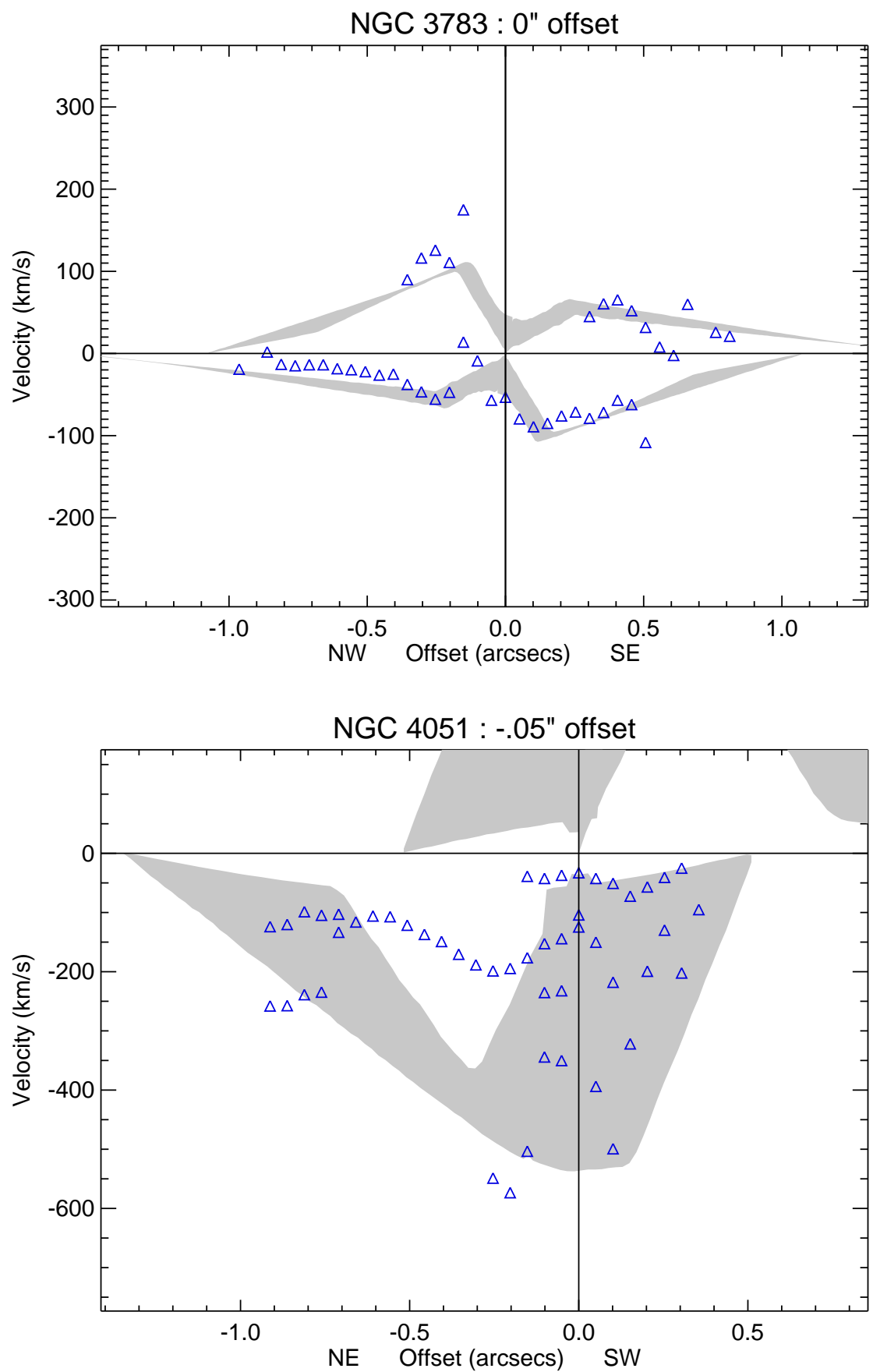

Figure D.9 

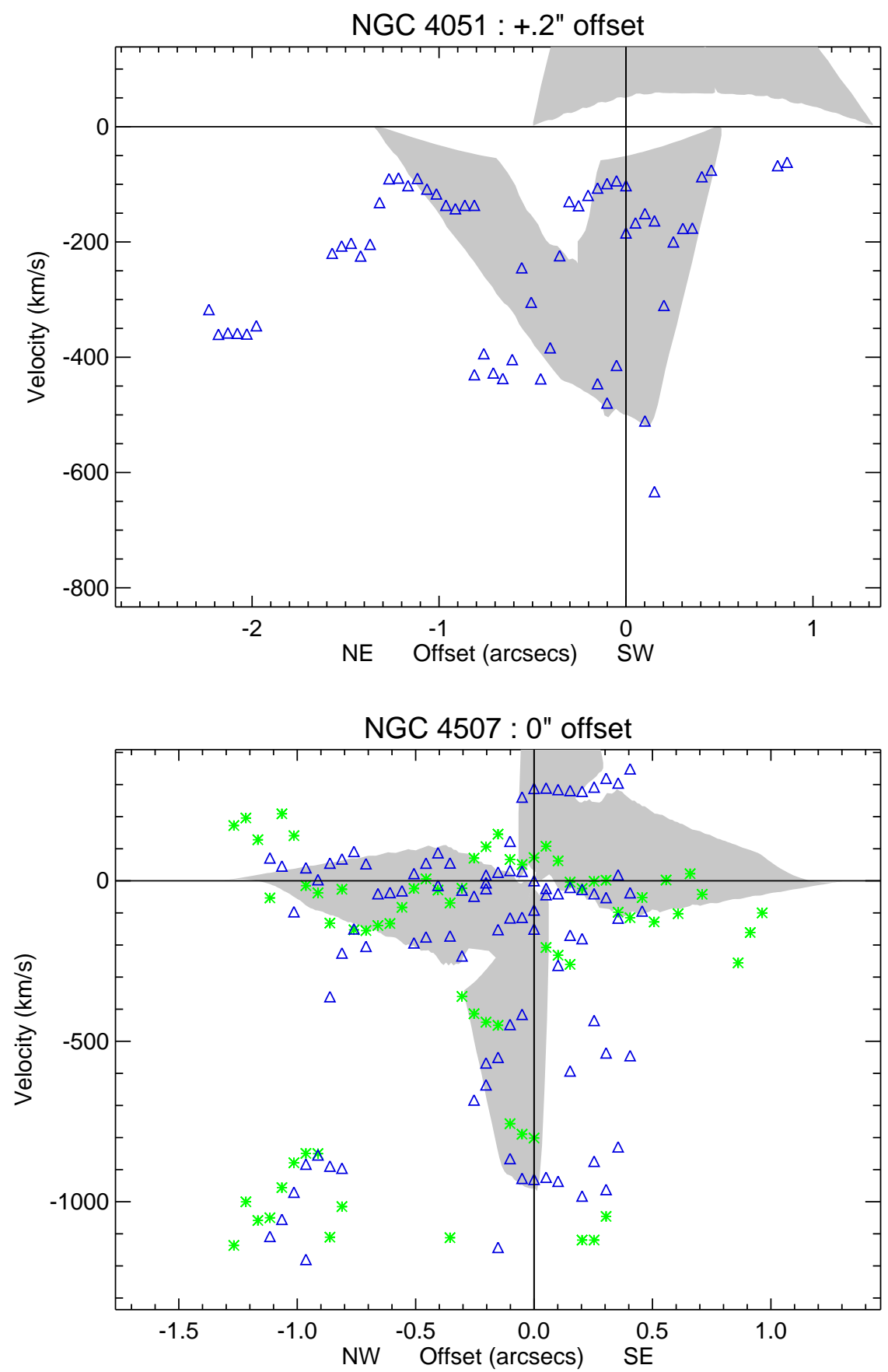

Figure D.10 

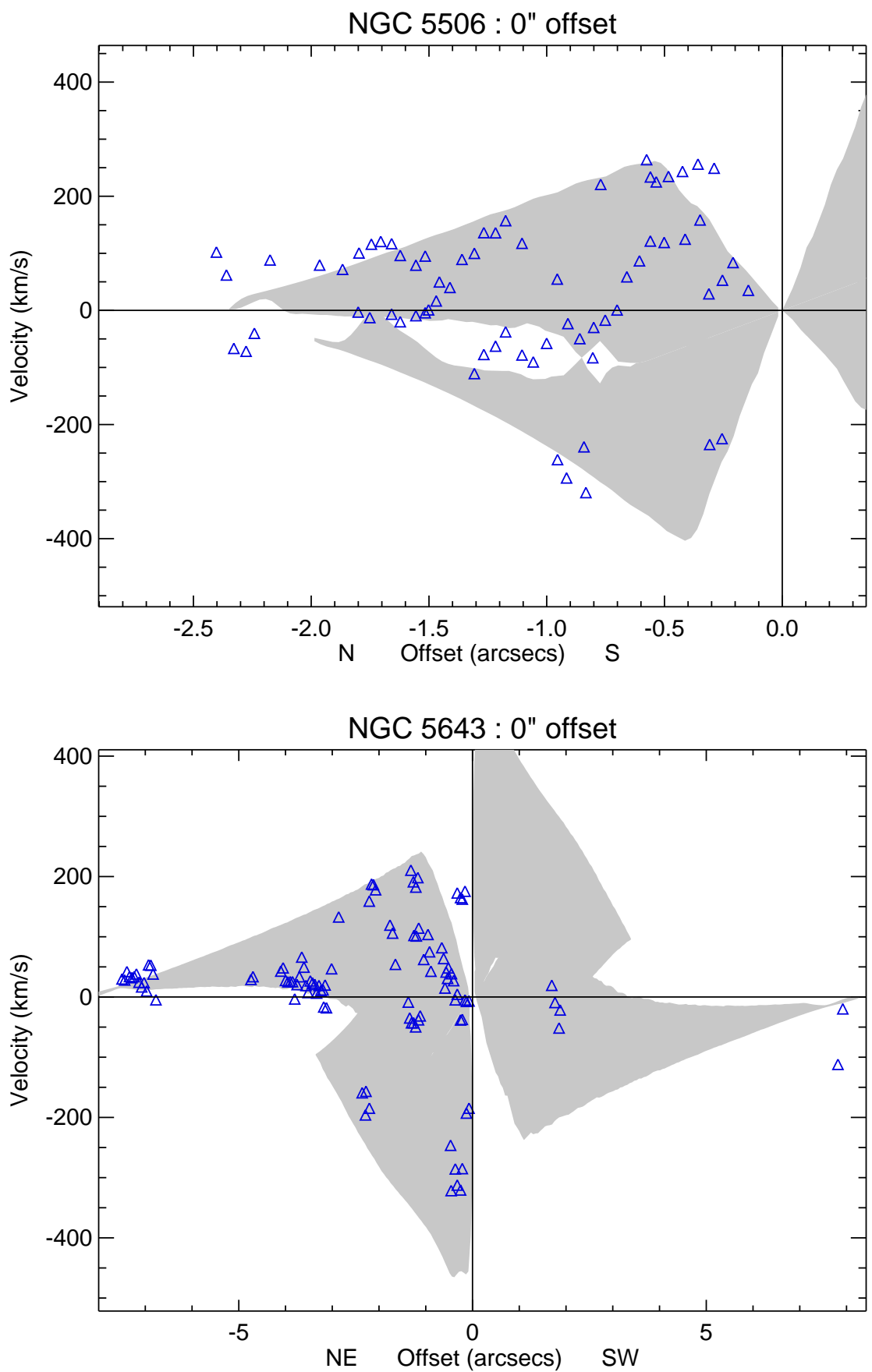

Figure D.11 

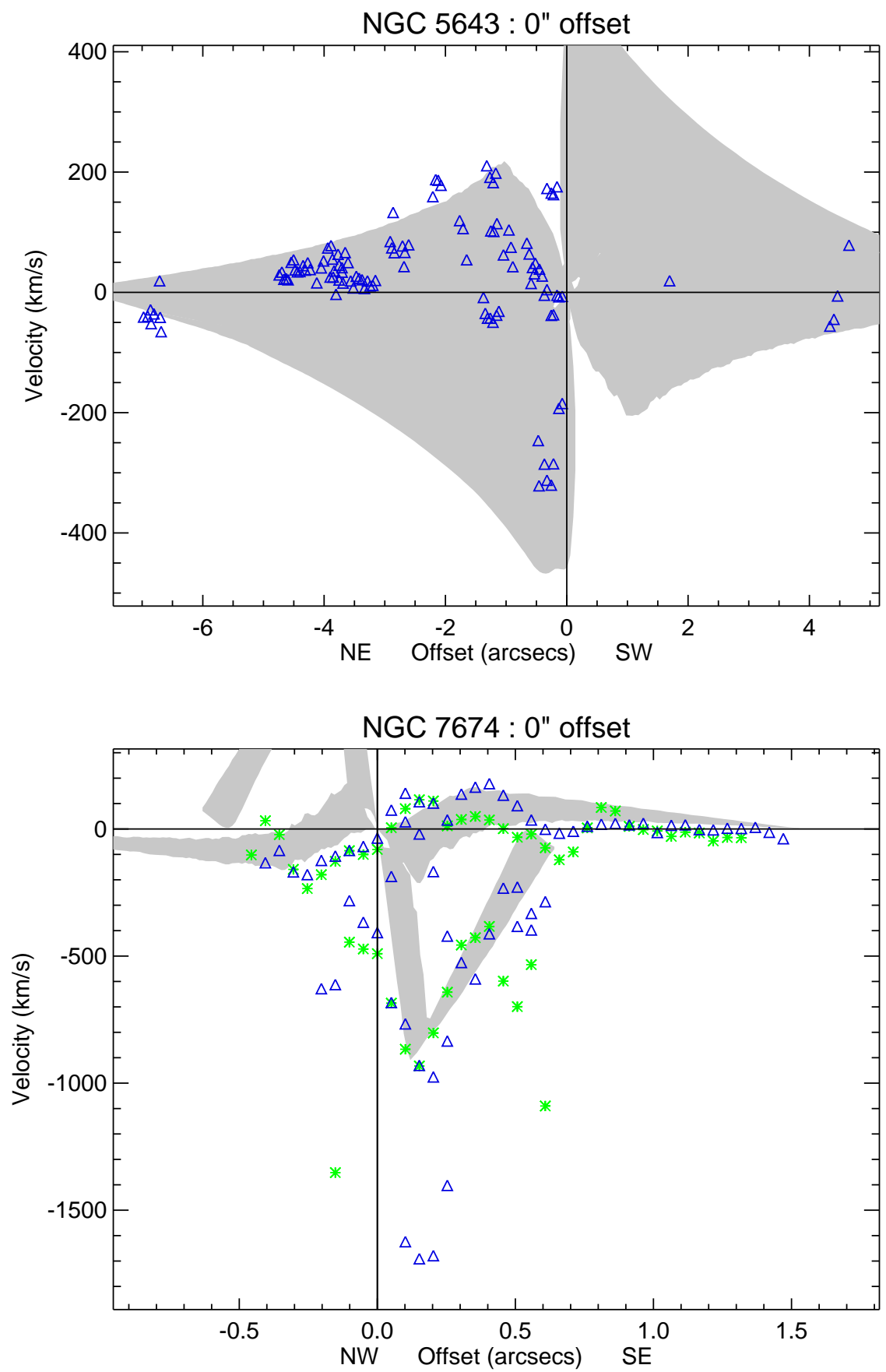

Figure D.12 